\title{
A NOVEL NON-PARAMETRIC DENSITY ESTIMATION APPROACH TO MEASURING MUON IONIZATION COOLING AND REVERSE EMITTANCE EXCHANGE IN THE MICE EXPERIMENT
}

BY

TANAZ ANGELINA MOHAYAI

Submitted in partial fulfillment of the requirements for the degree of Doctor of Philosophy in Physics in the Graduate College of the Illinois Institute of Technology

$$
\text { Approved } \frac{C \text { U/Pavel Snopok }}{\text { Advisor }}
$$

Chicago, Illinois

December 2018 
(c) Copyright by

TANAZ ANGELINA MOHAYAI

December 2018 


\section{ACKNOWLEDGMENT}

I am humbled and grateful to have made it to the end of my Ph.D. journey: completing my graduate research and writing this thesis could not have been possible were it not for the support of many people. This acknowledgment section serves as an opportunity to express my sincere and wholehearted gratitude to each person who made this thesis and its completion possible.

My Ph.D. advisor, Professor Pavel Snopok, provided me with the opportunity to live a productive Ph.D. life as a contributing member of the international MICE collaboration. He encouraged me to be bold in taking unconventional and creative steps in my analysis and helped me determine the direction of my research project, one that turned into a pioneering work in the application of the non-parametric density estimation technique to muon beam cooling. He took the time to teach me valuable

programming and physics skills and ensured that I have the opportunity to travel to physics conferences, workshops, and summer schools to further expand on these skills. He made a great effort in keeping me connected with our MICE collaborators by encouraging me to travel to Rutherford Appleton Laboratory to take shifts, serve as the BeamLine-On Call person, and participate in collaboration meetings.

Professor Dan Kaplan introduced me to MICE and helped me find a role within the collaboration and gain momentum in my contribution to the group. He made every effort to ensure that aside from software skills, I develop extensive experimental skills and ensured that I understand the physics behind MICE at a deep level. He took an interest in me as a person and helped me grow in confidence as a scientist. I am genuinely grateful for the valuable time he took to carefully review my papers, posters, and presentation slides and for making sure that I build on my communication skills by participating in conferences and workshops and delivering talks and posters that were of high quality. I am genuinely grateful for his mentorship and kindness 
throughout my Ph.D. life.

Professor David Neuffer guided me during my Department of Energy Office of Science Graduate Student Research (SCGSR) fellowship and acted as my Fermilab adviser throughout my Ph.D. journey. He made a great effort to ensure that all the resources were available for the completion of the SCGSR project and this thesis. I am grateful for the many conversations I have had with Dave where he shared great ideas and taught me to think outside the box.

I am grateful for the support of many experts at Illinois Tech, Fermilab, the University of Mississippi, and our worldwide MICE collaborators with whom I had the opportunity to work closely. Rarely does a graduate student have the opportunity to closely interact and learn from some of the most renowned experts. Thanks to Dr. Chris Rogers, for many valuable discussions and advice on the kernel density estimation analysis, for leading an excellent and efficient data-analysis team, for the opportunity to serve as a physics shifter, for his brilliant ideas, and for teaching me to take a creative approach to every concept and analysis subject within MICE; to Dr. Victoria Blackmore for her general kindness and support, for her mentorship, for a careful review of my many conference contributions, and for keeping me company with her wonderful stories in the control room during shifts; to Professor Yagmur Torun for his valuable insight, advice, and guidance on this thesis, and for ensuring that I was supported to travel to Rutherford Appleton Laboratory to take shifts and contribute to MICE, first-hand; to Dr. Durga Rajaram for answering my MAUS questions and for his support on the wedge simulations; to Dr. Milorad Popovic for his general kindness, support, and his leadership during MICE operations; to Professors Lucien Cremaldi, David Sanders, and Don Summers at the University of Mississippi for taking the lead in manufacturing the wedge absorber, for great conversations on muon physics while on shift at RAL, and for providing me with the opportunity to 
excite the Ole Miss Physics Department about MICE; to Dr. Paolo Franchini for his exceptional leadership during MICE operations, for the opportunity to perform beamline tuning in MICE, and for his mentorship; to Professor Alan Bross who led the US MICE analysis team, especially in bringing together all the expertise on the wedge absorber idea, which due to the efforts and supports of many, including Dave, Pavel, Dan, Milorad, David, Don, and Lucien, Chris, Paolo, Victoria, Yagmur, Durga had the chance to go from being a conceptual design to being installed in the MICE cooling channel for the demonstration of reverse emittance exchange; to Professor Domizia Orestano for her mentorship, general kindness, expertise on the PID detectors in MICE, and great company during shifts; to Professor Paul Soler for his expertise and advice on material studies and energy loss in the wedge absorber; to Dr. Mark Palmer for his tireless efforts in ensuring the academic well-being of every member of the Muon Accelerator Program and for commenting on several of my papers, presentation slides, and posters; to Professor Ken Long, MICE spokesperson, for his advice on this thesis work and for ensuring that I have a contributing role within the collaboration; to Dr. J. Scott Berg for valuable discussions and advice on the kernel density estimation analysis; to Dr. Steve Boyd for the opportunity to be involved in MICE data-taking and serve as a shifter; to Dr. Melissa Uchida for her general kindness, her expertise on the MICE trackers, and for great leadership during MICE operations; to Dr. Daniel Bowring for his advice on this thesis work and for helping me delve into the machine learning aspect of the density estimation technique; to Dr. Dimitrije Maletic, for his help with the wedge MC production; to Professor Vittorio Palladino, leading member of the MICE speaker's bureau, for the opportunity to represent the collaboration at several conferences and workshops; to Dr. Henry Nebrensky, the beamline expert, for training me as a shifter and a BeamLine-On Call expert and for his guidance while I served as a BeamLine-On Call person at RAL; to Dr. Ben Freemire, for his advice on the thesis and the next steps; to Dr. Ao Liu for many conversations on density 
estimation analysis and muon physics during shifts.

Where I stand, today could not have been possible had it not been for those who supported me early on in my physics career. In this regard, I would like to thank Professor Cristiano Galbiati, without whose support I would not be where I am today and would not have had the opportunity to work with an excellent research team at Princeton University and LNGS-INFN. He put faith in me and provided me with the opportunity to develop my skills as an experimental physicist. I am also grateful for the support of the CSUN physics community, including Professors Cristina Ana Cadavid, Say-Peng Lim, Nicholas Kioussis, and Debi Prasad Choudhary who provided me with the opportunity to grow in confidence in my physics life and learn from some of the most caring mentors.

The Illinois Tech Physics Department has made me feel part of a wonderful community, and I am grateful to all the professors and staff with whom I have interacted while I was a graduate student here. Thank you to Professor Carlo Segre, my first-year graduate advisor, for his valuable advice, guidance, and great mentorship; to Professor Yurii Shylnov for his general kindness, his advice, and support, his mentorship, for making graduate classes enjoyable, and for all that I learned from him during the first two years of my academic life at Illinois Tech; to Professor Alan Glodowski for the opportunity to serve as a teaching assistant; to Professor Linda Spentzouris for her general support and her advice on this work, as a member of my thesis committee; to Professor Kevin Cassel for his advice and support on this thesis work and for being a member of my thesis committee; to Dr. Patty Johnson Winston for her time and her advice on the thesis formatting and the required steps for graduation; and to Joy Calderone and Todd Kersh for all their support and many wonderful conversations. I am also grateful to the U.S. Particle Accelerator School, USPAS professors: thank you to professors Frank Zimmermann, Michiko Minty, and 
Alexander Chao for the opportunity to learn beam physics from some of the most renowned experts in the field.

I am grateful to the agencies and organizations that have provided support and funding for the research carried out in this thesis: to the DOE Office of Science Graduate Student Research (SCGSR) program for a great fellowship program and for providing me with the opportunity to work at Fermilab with a great advisor and mentor such as Dave Neuffer; to the IIT Starr and Fieldhouse research fellowship for the opportunity to carry out my research in collaboration with our Fermilab colleagues; and for the numerous travel, school tuition, and conference supports that have made my participation in various conferences and schools possible: the APS FGSA Excellence in Graduate Research award, Machine Learning in High Energy Physics school tuition and travel support, USPAS tuition and travel support, International Conference on High Energy Physics, ICHEP conference attendance support, International Particle Accelerator Conference, IPAC travel and conference attendance support, APS Division of Particles and Fields conference attendance support, and the North American Particle Accelerator conference attendance support.

I would also like to take the time to thank my friends, family, and the postgraduate, graduate, and undergraduate colleagues with whom I have had the pleasure to interact; this includes Shokoufeh Asalzadeh, Elahe Moazzen, Nicole Neveu, Adriana Manas, Rachel Seibert, Hilal Saglam, Jan Greis, Celeste Pidcott, Sophie Middleton, Chris Hunt, Mihailo Savic, Daniel Giles, Varrick Suezaki, Pranava Surukuchi, Yingpeng Song, Misha Fedorov, and many more. Special thanks to my family, especially my parents, for cultivating my early interest in physics, for always being there for me, for believing in me, and for being patient with me as I finished this phase of my life; to my parents-in-law, for always encouraging me to keep going and for their constant emotional support despite the fact that we are continents apart; to my grandmother 
for her enormous support, for constantly believing in me, and for being an enthusiastic listener whenever I talked about my work; to my grandfather who taught me hard work and encouraged me to be curious and never cease learning. I am truly sorry that he is not around to see me become a $\mathrm{Ph}$. D. He always encouraged me never to give up and to keep going, and I have no doubt that he has been there with me in every step of this process.

To my husband, Peyman, to whom I dedicate this thesis: words cannot describe how grateful I am to have you and your support in my life. Thank you for being a friend, for your utmost support, for always being there for me, for encouraging me to keep going, and for making sure we find time for short breaks. I could not have done this without you. The two of us have come a long way with a longer journey awaiting us at the end of this road, but I am always thrilled and excited to walk the toughest roads and climb the tallest mountains with you. 


\begin{abstract}
The Muon Ionization Cooling Experiment (MICE) located at Rutherford Appleton Laboratory in the U.K. has demonstrated muon ionization beam cooling for the first time. A beam of muons in MICE is produced from high-energy proton beam collision with a fixed target, generating pions which in turn decay into muons. Pion-decay muons, thus, are tertiary particles and, as a result, occupy a large volume in position-momentum phase space. To fit the muon beam into smaller and more cost-effective accelerating devices, muon beam phase-space volume needs to be reduced (beam cooling). Ionization beam cooling, which before MICE has never been demonstrated experimentally for muons, is the only technique fast enough to be used for muons within their short lifetime. Ionization cooling occurs when muons traverse an absorbing material and lose momentum through ionization energy loss. The cooling effect in MICE is measured using two scintillating-fiber tracking detectors. These trackers, one upstream and one downstream of the absorber, reconstruct and measure the position and momentum coordinates of each muon. Given the precision MICE needed to demonstrate beam cooling, it is necessary to develop analysis tools that can account for any effects that may lead to inaccurate measurement of cooling, such as non-linear effects in beam optics. Non-parametric density estimation techniques, such as kernel density estimation (KDE), provide a basis for creating analysis tools that are robust against these effects, directly calculating the muon beam phase-space density and volume for demonstrating beam cooling. This thesis focuses on the application of KDE to the measurement of beam cooling in MICE. The KDE technique is validated using known distributions and is applied to simulated and experimental MICE data corresponding to the various magnet, optics, and absorber configurations. Using the KDE technique, muon beam cooling in the four-dimensional transverse phase space, as well as reverse emittance exchange using MICE data have been demonstrated.
\end{abstract}




\section{TABLE OF CONTENTS}

Page

ACKNOWLEDGEMENT . . . . . . . . . . . . . . . iii

ABSTRACT ........................ ix

LIST OF TABLES . . . . . . . . . . . . . . . . . . xii

LIST OF FIGURES . . . . . . . . . . . . . . . . . . . . $\quad \mathrm{xx}$ CHAPTER

1. THE PHYSICS CASE: NEUTRINO FACTORY AND MUON COLLIDER . . . . . . . . . . . . . . . . . 1

1.1 Motivation: Understanding the Smallest Scale . . . . . . 1

1.2 Neutrino Physics: A Brief History and Introduction . . . . 3

1.3 Neutrino Factory . . . . . . . . . . . . . . . . . . 12

1.4 Muon Collider . . . . . . . . . . . . . . . . . . 16

2. MUON IONIZATION COOLING EXPERIMENT . . . . . . 22

2.1 The Principle . . . . . . . . . . . . . . . . . . . . . . . . 22

2.2 The Experiment $\ldots \ldots \ldots \ldots$

2.2 .1 Beamline. . . . . . . . . . . . . . . . . 38

2.2 .2 PID Detectors. . . . . . . . . . . . . 41

2.2.3 Trackers and the Cooling Channel Magnets. . . . . . 44

3. NON-PARAMETRIC DENSITY ESTIMATION: A NOVEL APPROACH TO MEASURING MUON BEAM COOLING . . . . 48

3.1 Motivation . . . . . . . . . . . . . . . . . . 48

3.2 Conceptual Foundation . . . . . . . . . . . . . . . . 48

3.2.1 Univariate Kernel Density Estimation. . . . . . . . 62

3.2.2 Multivariate Kernel Density Estimation. . . . . . . 63

3.3 Validation against Known Distributions . . . . . . . 64 
3.4 Summary . . . . . . . . . . . . . . . . . . . . . . . 70

4. NOVEL APPLICATION OF KDE IN SIMULATION . . . . . . 72

4.1 Motivation . . . . . . . . . . . . . . . . . 72

4.2 Test with a Quadrupole Magnet . . . . . . . . . . . . . 73

4.3 Simulated MICE Baseline Lattice . . . . . . . . . . . 76

4.4 Simulated MICE Lattice - a "least desirable" realistic case . 86

4.5 Simulated Cooling Performance with a Wedge-shaped Absorber 95

4.6 Summary . . . . . . . . . . . . . . . . . . 105

5. NOVEL APPLICATION OF KDE IN DATA . . . . . . . 107

5.1 LiH Flat Absorber Analysis . . . . . . . . . . . . . . 109

5.2 Polyethylene Wedge-shaped Absorber Analysis . . . . . 120

$5.3 \quad$ Summary . . . . . . . . . . . . . . . . . . . . 132

6. CONCLUSIONS AND SUMMARY . . . . . . . . . . 134

BIBLIOGRAPHY . . . . . . . . . . . . . . . . . . . 137 


\section{LIST OF TABLES}

Table

Page

4.1 Simulation parameters for studying a beam of muons of input emittance of $3 \pi \mathrm{mm} \cdot \mathrm{rad}$ (to keep the notation short, the units of emittance will be written as $\mathrm{mm}$ in what follows) and reference momentum of $200 \mathrm{MeV} / c$, traversing a quadrupole magnet. . . . . . . . . . . .

4.2 Currents of upstream SS and AFC as specified in G4beamline MICE lattice. The downstream spectrometer coils have the same currents with flipped signs. . . . . . . . . . . . . . . . . . . . . . . . .

4.3 Currents of upstream SS and AFC as specified in G4beamline MICE lattice. The downstream spectrometer coils have the same currents with flipped signs. . . . . . . . . . . . . . . . . . . . . . . .

4.4 Parameters of the G4beamline simulation for the emittance exchange studies. . . . . . . . . . . . . . . . . . . . . .

4.5 Currents of upstream and downstream SS and AFC coils as specified in G4beamline MICE lattice with a wedge absorber. . . . . . . . .

5.1 List of PID cuts applied to the $\mathrm{LiH}$ experimental and simulated data along with their respective selected values. . . . . . . . . . . . . 


\section{LIST OF FIGURES}

Figure $\quad$ Page

1.1 The standard model of particle physics. . . . . . . . . . . . . . 2

1.2 Schematic diagram of the physics behind the Cowan-Reines neutrino detection, demonstrating the electron-positron annihilation and the neutron thermalization in $\mathrm{CdCl}_{2}$-liquid scintillator solution [6]. . .

1.3 Mass hierarchy as illustrated for the two different cases of normal hierarchy (left) and inverted hierarchy (right). The terms $\Delta m_{\text {atm }}^{2} \approx$ $\left|\Delta m_{31}^{2}\right| \approx\left|\Delta m_{32}^{2}\right|$ and $\Delta m_{\text {sol }}^{2} \approx \Delta m_{21}^{2}$ are the atmospheric and solar mass-squared splittings, respectively [10]. . . . . . . . . . . . . 9

1.4 Schematic design of a neutrino factory [1] . . . . . . . . . . . . 14

1.5 Front end of the NF in one of its proposed designs [1]. . . . . . . 14

1.6 Expected precision for the measurement of the CP-violating phase, $\delta$, at various present and future neutrino facilities [23]. The International Design Study for the Neutrino Factory (IDS-NF) demonstrates the best precision for the measurement of the CP-violation.

1.7 Luminosity per electrical power (power consumed by the collider facility) versus center of mass energy for various proposed collider

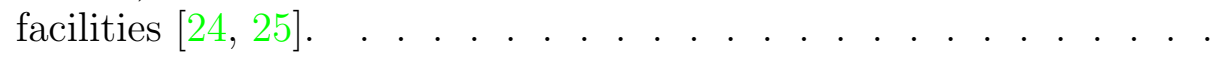

1.8 Muon collider and neutrino factory schematic designs compared. The two facilities can share the same complex and be built on the same experimental site [27]. . . . . . . . . . . . . . . . . . . .

2.1 A 2016 photo of the Muon Ionization Cooling Experiment, MICE, in its final configuration showing partial return yoke surrounding the upstream and downstream Spectrometer Solenoid and Absorber Focus Coil modules. . . . . . . . . . . . . . . . . . . . . .

2.2 A 2015 photo of the Muon Ionization Cooling Experiment, MICE, in its final configuration prior to the installation of the partial return yoke, showing the upstream and downstream Spectrometer Solenoid and the Absorber Focus Coil modules. . . . . . . . . . . . . . .

2.3 Ionization energy loss rate vs, the muon momentum [9]. . . . . .

2.4 Effect of multiple scattering on the particle track as it travels a distance $x$ through material [9]. . . . . . . . . . . . . . . . . .

2.5 An illustration of the motion of the reference particle in an accelerator ring. . . . . . . . . . . . . . . . . . . . . . 
2.6 The 2D trace space ellipse. The parameters of this ellipse are the Twiss parameters [35]. . . . . . . . . . . . . . . . . . .

2.7 Ionization energy loss in material reduces the beam momentum in all directions (1), and the subsequent acceleration from RF cavity replaces the longitudinal momentum (2). The energy loss is concurrent with the multiple scattering (3) which in turn heats the beam by slightly changing the direction of the momentum vector [28]. . .

2.8 Process of emittance exchange via a wedge-shaped absorber. Courtesy of Muons, Inc. [37] . . . . . . . . . . . . . . . . . . . . .

2.9 Process of reverse emittance exchange via a wedge-shaped absorber. Courtesy of Muons, Inc. [37]. . . . . . . . . . . . . . . . . . . 38

2.10 Schematics of the MICE muon beamline. . . . . . . . . . . . .

2.11 Schematic diagram of MICE in its final configuration with upstream and downstream spectrometers surrounding a cooling cell, and particle identification detectors (ToF, Cherenkov, KL, and EMR). . .

2.12 MICE beam downstream of decay solenoid. The green rectangle represents the "muonic" beam setting on the figure; this beam almost purely consists of muons. The "pionic" beam setting, on the other hand, has approximately equal numbers of pions and muons with momenta in the range of 400 to $430 \mathrm{MeV} / c$ [39]. . . . . . .

2.13 Three distinct time-of-flight peaks for electrons, muons, and pions using the time-of-flight of the particles between ToF0 and ToF1. A "pionic" beam was used to produce this plot. . . . . . . . . . . .

2.14 Two distinct time-of-flight peaks for electrons and muons/pions using the time of flight of the particles between ToF0 and ToF1. A muonic beam was used to produce this plot. To better distinguish between pions and muons, other PID detectors must be used. . . .

2.15 The ADC responses of muons and pions as they interact with the KL detector. The ADC distribution indicates that pions have larger tails compared to muons and that the majority of the beam particles in the MICE beam are muons, closely following the muon template. The pi/mu beam is a pionic beam [40]. . . . . . . . . . . . . . .

2.16 Muon and electron plane density distributions in the EMR [41]. . 43

2.17 MICE scintillating fiber tracker. . . . . . . . . . . . . . . . . . 45

2.18 The layout and orientation of the doublet fiber layers in the MICE trackers [42]. . . . . . . . . . . . . . . . . . . . . . . . . . . 
2.19 Photos of the SS module sitting inside the partial return yoke (left) and the AFC module (right). The AFC module is typically covered by the partial return yoke but at the time of the photo, it was removed (absorber changed in progress). . . . . . . . . . . . . .

2.20 Momentum reconstruction in the transverse plane (upper diagram) and the longitudinal direction (lower diagram). $r$ represents the radius of the helix $\left(r=\frac{p_{\perp}}{q B}\right)$. The same track would have a sinosoidal shape in the longitudinal direction. . . . . . . . . . . . . . .

3.1 Histogram of the simulated muon $x$ positions at the entrance to the MICE cooling channel with different choices of $x_{0}(\mathrm{a})$ and bin width $h$ ( $h$ is) (b). For illustration purposes, the sample size is $50 . \quad$. . .

3.2 An illustration of the KDE technique in one dimension; the bandwidth parameter is 0.4 and Gaussian kernel functions are used. . .

3.3 The $x$ distribution is approximately Gaussian with the optimal bandwidth parameter, $h=0.3$. When the bandwidth parameter is 3.0 , the estimated PDF is over-smoothed (green curve). A smaller value, 0.03, at the bandwidth parameter leads to a noisier estimated density (red curve) [48]. . . . . . . . . . . . . . . . . . . . . . .

3.4 The "true" distribution is randomly drawn from a Gaussian. As expected, green curve has minimized mean integrated square error [48]. . . . . . . . . . . . . . . . . . . . . . . .

3.5 The true and KDE $x$ distributions containing 1000 data points. The data points are drawn from a Gaussian distribution 10 times. The red curve(s) are the KDE density curve(s) while the green curve(s) are the true $\mathrm{PDF}(\mathrm{s})$. The left-hand side plots are the 10 random samples of the true density and the KDE density, while the righthand side plots are their averages. . . . . . . . . . . . . . . . .

3.6 The true and KDE $x$ distributions containing 10,000 data points. The data points are drawn from a Gaussian distribution 10 times. The red curve(s) are the KDE density curve(s) while the green curve(s) are the true $\operatorname{PDF}(\mathrm{s})$. The left-hand side plots are the 10 random samples of the true density and the KDE density, while the right-hand side plots are their averages. . . . . . . . . . . . . .

3.7 The true and KDE $x$ distributions containing 100,000 data points. The data points are drawn from a Gaussian distribution 10 times. The red curve(s) are the KDE density curve(s) while the green curve(s) are the true $\operatorname{PDF}(\mathrm{s})$. The left-hand side plots are the 10 random samples of the true density and the KDE density, while the right-hand side plots are their averages. . . . . . . . . . . . . . 
3.8 The variation of the bandwidth factorr, $h_{\text {factor }}$ with the sample size.

3.9 An illustration of why the bandwidth parameter, $h$ should depend on sample size. $h$ is fixed across the three contours. The contours represent the distribution of the simulated muon beam at the entrance of the MICE upstream tracker. The sample sizes change from $1000 \rightarrow 10,000 \rightarrow 100,000$ going from left to right. $h$ is too small for the smallest sample size of 1000 (left) and optimal for the largest sample size of 100,000 (right). . . . . . . . . . . . . . . . . . .

$4.1\left(x, p_{x}\right)$ (top) and $\left(y, p_{y}\right)$ (bottom) phase-space contours (drawn by contour lines) for a muon beam distribution going from an upstream (left) to a downstream point (right) inside a quad magnet. The quadrupole is focusing along $x$ and de-focusing along $y$, hence the asymmetry between $x$ and $y$ planes. . . . . . . . . . . . . . . .

4.2 The contour density vs. beam area, from an upstream point in the quadrupole magnet to a downstream point, for the 2D (left) $\left(x, p_{x}\right)$ and (right) $\left(y, p_{y}\right)$ beam distributions. The slight discrepancy between upstream and downstream curves in the $\left(y, p_{y}\right)$ distribution is an artifact of the limited number of density contours considered.

4.3 Plots of the on-axis magnetic field (upper left), average momentum (upper right), and emittance (lower middle) for the MICE baseline lattice study. Note the decrease across the absorber for all three plots. The B-field at the exit of the upstream tracker is at $4 \mathrm{~T}$ and flips sign at the absorber to minimize the build-up of the net angular momentum. . . . . . . . . . . . . . . . . . . . 77

4.4 Phase-space contours before and after the LiH absorber [48]. . . . 79

4.5 Density versus the average beam radius in the 4D phase-space. The density corresponds to the phase-space density of a contour of constant density while the volume is the volume enclosed inside this contour. The contour corresponding to the core of the beam has a mean radius value of $0.02\left[\mathrm{~m}^{1 / 2}(\mathrm{GeV} / c)^{1 / 2}\right]$ [48]. . . . . . . . . .

4.6 Evolution of the density of the $9^{\text {th }}$-percentile contour for two absorber configurations: $65 \mathrm{~mm} \mathrm{LiH}$ absorber and no absorber. The increase in density from the upstream $(z \approx-1.8 \mathrm{~m})$ to the downstream $(z \approx 1.8 \mathrm{~m})$ tracker reference plane (vertical dashed green lines) is a result of beam cooling. . . . . . . . . . . . . . . . 
4.7 Evolution of the volume of the $9^{\text {th }}$-percentile contour for two absorber configurations of $65 \mathrm{~mm} \mathrm{LiH}$ absorber and no absorber. The reduction in volume from the upstream $(z \approx-1.8 \mathrm{~m})$ to the downstream $(z \approx 1.8 \mathrm{~m})$ tracker reference planes (vertical dashed green lines) are the results of beam cooling. . . . . . . . . . . . . . .

4.8 A three-dimensional illustration of the volume calculation using the $\mathrm{MC}$ approach. The MC points are generated uniformly within the three-dimensional bounding box. Only the MC points that reside within the bounded contour (red points) are used to calculate the contour volume. . . . . . . . . . . . . . . . . . . . . .

4.9 The schematics of the MICE displaying the locations of the superconducting coils in the SS and AFC. . . . . . . . . . . . . . . .

4.10 Evolution of the density (a) and volume (b) of the $9^{\text {th }}$-percentile contour for two absorber configurations of $65 \mathrm{~mm} \mathrm{LiH}$ absorber and no absorber. The changes in density and volume from the upstream $(z \approx-1.8 \mathrm{~m})$ to the downstream $(z \approx 1.8 \mathrm{~m})$ tracker reference planes (vertical dashed green lines) are the results of beam cooling (6-140 beam setting) [52]. . . . . . . . . . . . . . . . . .

4.11 Evolution of the density (a) and volume (b) of the $9^{\text {th }}$-percentile contour for two absorber configurations of $65 \mathrm{~mm} \mathrm{LiH}$ absorber and no absorber. The changes in density and volume from the upstream $(z \approx-1.8 \mathrm{~m})$ to the downstream $(z \approx 1.8 \mathrm{~m})$ tracker reference planes (vertical dashed green lines) are the results of beam cooling (10-140 setting) [52]. . . . . . . . . . . . . . . . .

4.12 An illustration of the amplitude concept in the $x-p_{x}$ phase space. The contours of constant density are drawn using the kernel estimated density. The black dots represent the muons in the phase space, and a constant amplitude contour is shown in white. . . . .

4.13 Density versus amplitude for the MICE lattice with 6-140 input beam setting [52]. . . . . . . . . . . . . . . . . . . .

4.14 Density versus amplitude for the MICE lattice with 10-140 input beam setting [52]. . . . . . . . . . . . . . . . . . . . . .

4.15 Evolution of the density (a) and volume (b) of the $2^{\text {nd }}$-percentile contour for the wedge absorber simulation. The $6 \mathrm{D}$ density and volume changes from the upstream $(z \approx-1.8 \mathrm{~m})$ to the downstream $(z \approx 1.8 \mathrm{~m})$ tracker reference planes (vertical dashed green lines). . 
4.16 Evolution of the density (a) and volume (b) of the $24^{\text {th }}$-percentile contour for the wedge absorber simulation. The changes in density and volume from the upstream $(z \approx-1.8 \mathrm{~m})$ to the downstream $(z \approx 1.8 \mathrm{~m})$ tracker reference planes (vertical dashed green lines) are the results of longitudinal beam cooling. . . . . . . . . . . .

4.17 Evolution of the 4D density (a) and volume (b) of the $9^{\text {th }}$-percentile contour for the wedge absorber simulation. The changes in transverse density and volume from the upstream $(z \approx-1.8 \mathrm{~m})$ to the downstream $(z \approx 1.8 \mathrm{~m})$ tracker reference planes (vertical dashed green lines) are the results of transverse beam heating. . . . . . .

4.18 Evolution of the density (a) and volume (b) of the $2^{\text {nd }}$-percentile contour. The changes in density and volume from $z \approx-1.8 \times 10^{3} \mathrm{~mm}$ to $z \approx 1.8 \times 10^{3} \mathrm{~mm}$ tracker reference plane (vertical dashed green lines) are the results of $6 \mathrm{D}$ heating or reverse emittance exchange.

4.19 Evolution of the density (a) and volume (b) of the $24^{\text {th }}$-percentile contour for wedge simulation. The changes in density and volume from the upstream $\left(z \approx-1.8 \times 10^{3} \mathrm{~mm}\right)$ to downstream $(z \approx 1.8 \times$ $10^{3} \mathrm{~mm}$ ) tracker reference plane (vertical dashed green lines) are the results of beam heating in the longitudinal direction. . . . . . . .

4.20 Evolution of the density (a) and volume (b) of the $9^{\text {th }}$-percentile contour for the wedge absorber simulation. The changes in density and volume from the upstream to downstream of the wedge (vertical dashed green lines) are the results of transverse beam cooling. .

5.1 The total number of particle triggers as recorded by the ToF1 detector in the course of MICE operation [54]. . . . . . . . . . . . 108

5.2 The typical MICE data structure [56]. . . . . . . . . . . . . . . 109

5.3 ToF versus upstream (upper plots) and downstream (lower plots) tracker momentum before (leftmost plots) and after (rightmost plots) the PID cuts applied on MICE data; these cuts are used to select the ToF and momenta corresponding to a muon beam in data. The cuts follow the MICE analysis convention. Note that the colorbars are the counts, normalized to the upstream total muon count (hence the same maximum count in all plots). . . . . . . . . . . . . . .

5.4 ToF versus upstream (upper plots) and downstream (lower plots) tracker momentum before (leftmost plots) and after (rightmost plots) the PID cuts applied on MC recon sample; these cuts are used to select the ToF and momenta corresponding to a muon beam in $\mathrm{MC}$ recon. The cuts follow the MICE analysis convention. . . . . . 
5.5 Comparison of the distributions in $x$ (upper plots) and $y$ (lower plots) before (left) and after (right) the LiH absorber (at the upstream and the downstream tracker reference planes) in simulation (MC recon) and (MICE experimental) data. . . . . . . . . . . .

5.6 Comparison of the distributions in $p_{x}$ (upper plots) and $p_{y}$ (lower plots) before (left) and after (right) the $\mathrm{LiH}$ absorber in simulation (MC recon) and (MICE experimental) data. . . . . . . . . . .

5.7 Phase-space plots of the upstream data sample. . . . . . . . . . 115

5.8 Phase-space plots of the downstream data sample. . . . . . . . . 115

5.9 Phase-space plots of the upstream MC recon sample. . . . . . . . 116

5.10 Phase-space plots of the downstream MC recon sample. . . . . . 116

5.11 Contour density versus the average beam radius in the transverse (upper plot) and the longitudinal direction (lower plot) in data; the increase in the beam core density in the transverse direction and decrease in the core density in the longitudinal direction are the expected beam cooling signals from a flat absorber. . . . . . . .

5.12 Contour density versus the average beam radius in the transverse (upper plot) and the longitudinal direction (lower plot) in $\mathrm{MC}$ recon; the increase in the beam core density in the transverse direction and decrease in the core density in the longitudinal direction are the expected beam cooling signals from a flat absorber. . . . . . . .

5.13 Photo of the wedge absorber installed inside the Focus Coil before insertion in the beam.

5.14 ToF vs. upstream (upper plots) and downstream (lower plots) tracker momentum before (left plots) and after (right plots) the PID cuts; these cuts are used to select the ToF and momenta corresponding to a muon beam in data and follow the MICE convention. . . .

5.15 ToF vs. upstream (upper plots) and downstream (lower plots) tracker momentum before (leftmost plots) and after (rightmost plots) the PID cuts; these cuts are used to select the ToF and momenta corresponding to a muon beam in MC recon and follow the MICE analysis convention. . . . . . . . . . . . . . . . . . . . . . . . . . .

5.16 The wedge absorber introduces an $x-p_{z}$ correlation in the MICE experimental beam (data); this is the result of reverse emittance exchange. . . . . . . . . . . . . . . . . . . . . . . . . . . . 
5.17 The wedge absorber introduces an $x-p_{z}$ correlation in the MICE simulated beam (MC recon); this is the result of reverse emittance exchange. . . . . . . . . . . . . . . . . . . . . . . .

5.18 Comparison of the distributions in $x$ (upper plots) and $y$ (lower plots) before (left) and after (right) the LiH absorber (at the upstream and the downstream tracker reference planes) in simulation (MC recon) and (MICE experimental) data. . . . . . . . . . . .

5.19 Comparison of the distributions in $p_{x}$ (upper plots) and $p_{y}$ (lower plots) before (left) and after (right) the $\mathrm{LiH}$ absorber in simulation (MC recon) and (MICE experimental) data. . . . . . . . . . .

5.20 Comparison of the energy distributions upstream (upper plots) and downstream (lower plots) of the wedge absorber in data and MC recon. . . . . . . . . . . . . . . . . . . . . . 126

5.21 Phase-space plots of the upstream data sample. . . . . . . . . . 127

5.22 Phase-space plots of the downstream data sample. . . . . . . . . 128

5.23 Phase-space plots of the upstream MC recon sample. . . . . . . . 128

5.24 Phase-space plots of the downstream MC recon sample. . . . . . 129

5.25 Contour density versus the average beam radius in the transverse plane (upper plot) and longitudinal plane (lower plot) in data; the increase in the beam core density in the transverse plane and decrease in density in the longitudinal plane are the expected beam cooling signals with a wedge absorber. . . . . . . . . . . . .

5.26 Contour density versus the average beam radius in the transverse plane (upper plot) and longitudinal plane (lower plot) in $\mathrm{MC}$ recon; the increase in the beam core density in the transverse plane and decrease in density in the longitudinal plane are the expected beam cooling signals with a wedge absorber. . . . . . . . . . . . . . 


\section{CHAPTER 1}

\section{THE PHYSICS CASE: NEUTRINO FACTORY AND MUON COLLIDER}

\subsection{Motivation: Understanding the Smallest Scale}

What are the underlying building blocks that make up our universe? Attempts have been made to answer this fundamental question, its answer having evolved from the four elements of air, earth, fire, and water first specified by the $4^{\text {th }}$ century B.C. Greek philosopher Empedocles (490-430 B.C.) to the elementary particles that have been studied since the discovery of muons (elementary particles central to this thesis) by Carl D. Anderson and Seth Neddermeyer in the 1930s. Today, the elementary particles are understood to be the most fundamental constituents of matter: quarks (making up protons and neutrons), leptons (e.g., electrons), gauge bosons (carriers of fundamental forces), and the Higgs boson. They are known to interact via the four fundamental forces of gravity, the strong force (responsible for holding the nucleons together), the weak force (responsible for the radioactive decay of an unstable nucleus), and electromagnetism. To model the fundamental laws of nature in the context of these elementary particles, a theory known as the Standard Model (SM) of particle physics has been developed. Figure 1.1 displays the elementary particles and their classification within the SM.

Although the SM has been successful in predicting the existence of numerous particles prior to their discovery (e.g., top quark, Higgs boson) and explaining most experimental observations and natural phenomena, it is still a work-in-progress, incapable of explaining certain phenomena such as neutrino oscillation (Section 1.2), dark matter and dark energy, and the matter-antimatter asymmetry in the universe. These phenomena can be explained if an extension is implemented in the SM (be- 


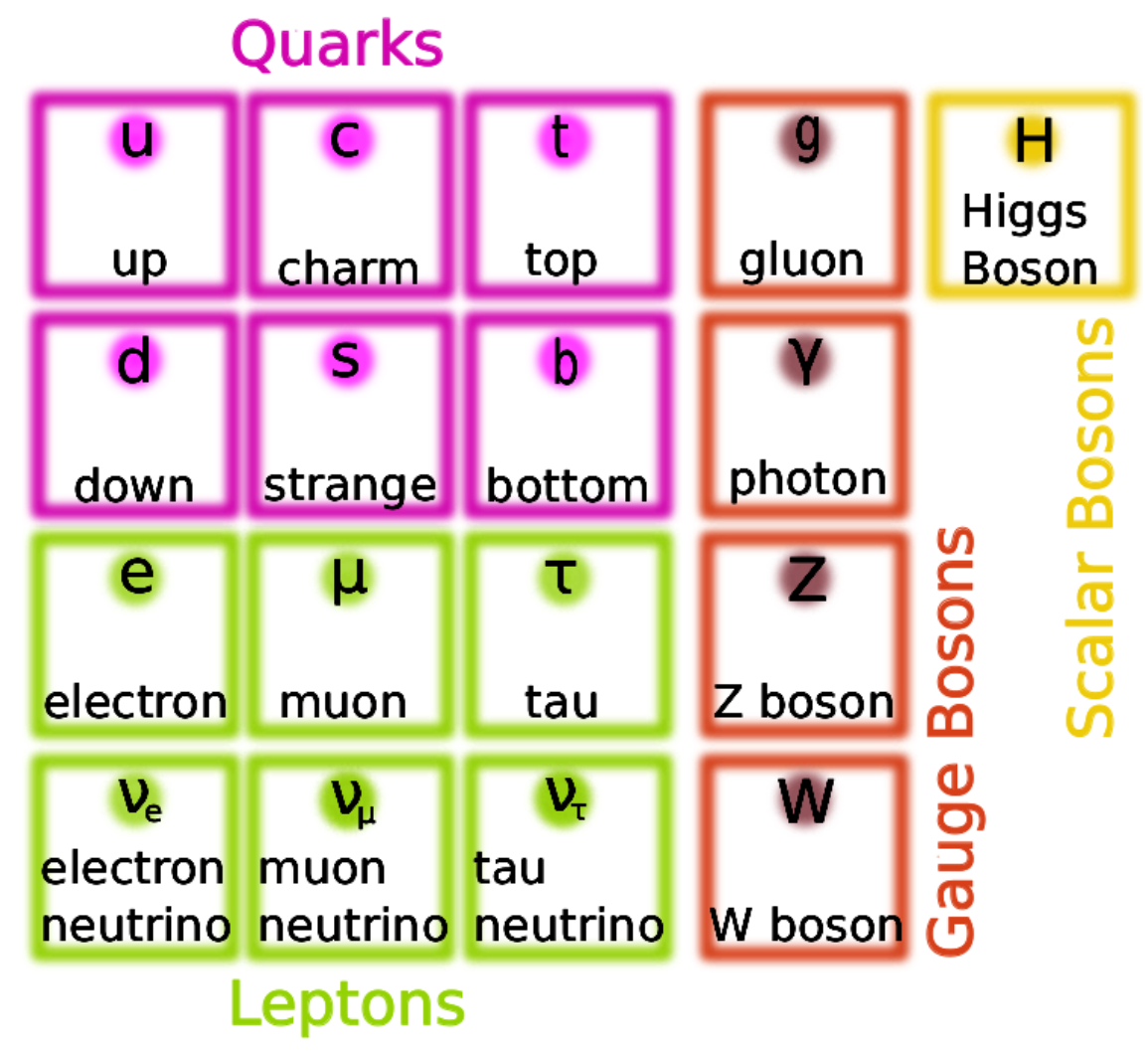

Figure 1.1. The standard model of particle physics.

yond the SM, or BSM), and understanding them requires more powerful experiments capable of probing the elementary particles and their properties more precisely. Examples of such experiments are a neutrino factory (NF) [1] probing the neutrino sector (Section 1.3) and a multi-TeV muon collider (MC) [2] searching for answers to the main questions in particle physics (Section 1.4). Chapter 2 covers the Muon Ionization Cooling Experiment (MICE), which is the proof-of-principle experiment for these future facilities. 


\subsection{Neutrino Physics: A Brief History and Introduction}

The discovery of radioactivity ${ }^{1}$ by Henri Becquerel in 1896, followed by Rutherford's classification of the beta decay $(\beta \text {-decay })^{2}$ in 1899, laid the foundation for the discovery of neutrinos. In 1914, James Chadwick observed that the energy distribution in $\beta$-decay has a broad and continuous spectrum as opposed to a sharp peak; this was a conservation of energy problem and was suggestive of an undetected particle, with a minimal mass and no electric charge, responsible for taking away part of the energy in the decay process. This undetected particle, named a neutron at the time, was first postulated by Wolfgang Pauli in 1930 as a "desperate remedy" to the conservation-of-energy problem in the $\beta$-decay process [3]. In 1932, however, following James Chadwick's discovery of the more-massive neutron (a constituent of the nucleus), Pauli's neutron was renamed "neutrino" by Enrico Fermi, who followed Edoardo Amaldi's first use of the name.

Neutrinos can be detected only via the neutral current (by means of Z boson exchange) or charged current (by means of $\mathrm{W}$ boson exchange) weak interaction. ${ }^{3}$ They are the only elementary particles that are detectable via only one of the fundamental forces (weak interaction). As a result, they were not discovered for another 25 years after their postulation by Pauli [4]. Frederick Reines and Clyde L. Cowan, Jr. believed that they could prove the existence of neutrinos via inverse $\beta$-decay, a reaction in which electron anti-neutrinos (the antiparticle of the electron neutrino) scatter off protons and produce neutrons and positrons (positively charged antiparticles of

\footnotetext{
${ }^{1}$ Radioactivity is the process by which an unstable nucleus decays, emitting, e.g., an electron, He nucleus, or photon.

${ }^{2}$ Beta decay is the process by which an unstable nucleus decays and emits an electron.

${ }^{3}$ Weak interaction or force is the mechanism behind the radioactive decay of an unstable nucleus.
} 
electrons),

$$
\overline{\nu_{e}}+p \rightarrow e^{+}+n
$$

The positrons from Equation $1.1\left(e^{+}\right)$would then annihilate with electrons, leading to the production of two photons $(\gamma)$,

$$
e^{+}+e^{-} \rightarrow 2 \gamma
$$

while the neutrons from Equation $1.1(n)$ would be captured by the nucleus, a process that is typically delayed by several microseconds (delayed coincident event). An example of such an interaction is neutron capture by the ${ }^{108} \mathrm{Cd}$ (cadmium) nucleus, which leads to $\gamma$ production,

$$
n+{ }^{108} \mathrm{Cd} \rightarrow{ }^{109} \mathrm{Cd}^{*} \rightarrow{ }^{109} \mathrm{Cd}+\gamma
$$

$\left({ }^{109} \mathrm{Cd}^{*}\right.$ is an excited state of $\left.{ }^{109} \mathrm{Cd}\right)$.

Reines and Cowan searched for a neutron capture signal and were able to prove the existence of (anti)neutrinos in 1956. They used a nuclear reactor as their neutrino source, and their initial experimental setup consisted of a tank of liquid scintillator mixed with $\mathrm{CdCl}_{2}$ (cadmium chloride), surrounded by photomultiplier tubes (PMTs). This initial design, placed at the Hanford site located in the state of Washington, suffered from a significant cosmic-ray background. To shield against cosmic rays, the experiment was relocated to the Savannah River site located in the state of South Carolina, 12 meters underground. The experiment adopted a new design at its new location and consisted of tanks of water-cadmium chloride solution as the interacting medium, sandwiched between tanks of liquid scintillator, surrounded by PMTs. With the new experimental setup, Cowan and Reines observed three delayed coincident events per hour [5], a clear sign of the inverse $\beta$-decay, and hence of the existence of the electron antineutrino (Fig. 1.2). The Cowan-Reines neutrino experiment was the first to detect the neutrino directly. Further experimental searches followed Reines 


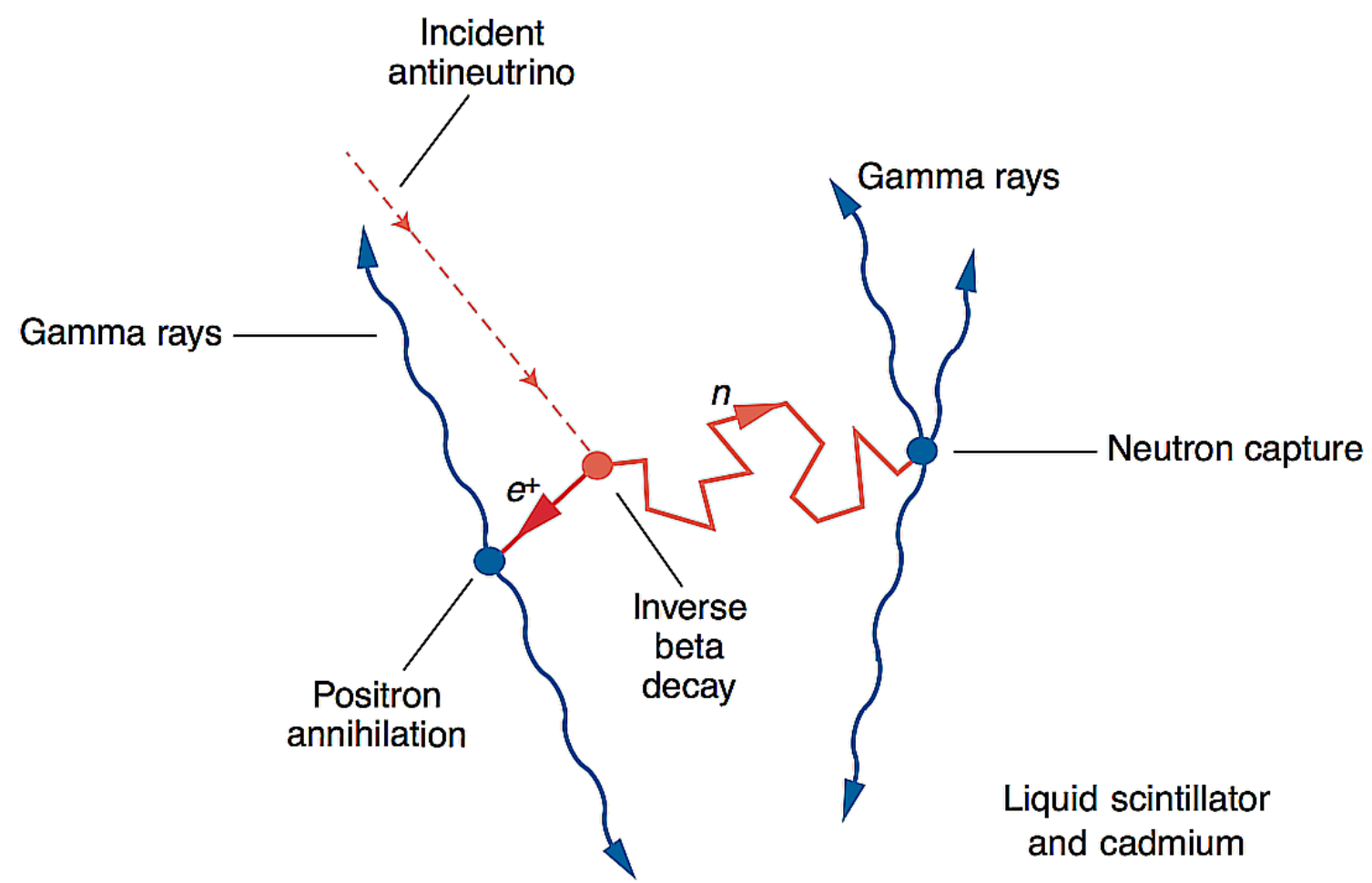

Figure 1.2. Schematic diagram of the physics behind the Cowan-Reines neutrino detection, demonstrating the electron-positron annihilation and the neutron thermalization in $\mathrm{CdCl}_{2}$-liquid scintillator solution [6].

and Cowan's discovery, including the experiments that led to the discoveries of the muon neutrino, $\nu_{\mu}$, by Leon Lederman, Melvin Schwartz, and Jack Steinberger at Brookhaven National Lab in 1962 [7], and the tau neutrino, $\nu_{\tau}$, by the DONUT collaboration at Fermilab in 2000 [8].

Neutrinos are produced in weak interactions, either with or absorbed to give, a charged lepton. According to the SM, there are three neutrino flavor eigenstates: the electron neutrino, $\nu_{e}$, named after the electron $(e)$; the $\nu_{\mu}$, named after the muon $(\mu)$; the $\nu_{\tau}$, named after the tau $(\tau)$ (Fig. 1.1). In general, within the SM there are six known quark flavors (up, down, top, bottom, charm, and strange) and six known lepton flavors $\left(e, \mu, \tau, \nu_{e}, \nu_{\mu}\right.$, and $\left.\nu_{\tau}\right)$ as shown in Figure 1.1). In addition to flavor states, neutrinos are characterized by their mass eigenstates $\left(\nu_{1}, \nu_{2}, \nu_{3}\right)$. The neutrino mass and flavor states are related via a mixing matrix known as the PMNS matrix 
(Pontecorvo-Maki-Nakagawa-Sakata), $U$ [4]. The flavor eigenstates, $\alpha$, are written as quantum superpositions of mass eigenstates, $i$, using the elements of the PMNS matrix, $U$,

$$
\left|\nu_{\alpha}\right\rangle=\sum_{i} U_{\alpha i}^{*}\left|\nu_{i}\right\rangle
$$

where ${ }^{*}$ represents the complex conjugate. Similarly, the mass eigenstates, $i$, are written as superpositions of flavor eigenstates, $\alpha$,

$$
\left|\nu_{i}\right\rangle=\sum_{i} U_{\alpha i}\left|\nu_{\alpha}\right\rangle
$$

where $\left|\nu_{\alpha}\right\rangle$ is a neutrino with a definite flavor $\left(\nu_{e}, \nu_{\mu}, \nu_{\tau}\right)$ and $\left|\nu_{i}\right\rangle$ a neutrino with a definite mass, $m_{i}(i=1,2,3)$. An example is the electron neutrino, $\nu_{e}$ :

$$
\left|\nu_{e}\right\rangle=U_{e 1}^{*}\left|\nu_{1}\right\rangle+U_{e 2}^{*}\left|\nu_{2}\right\rangle+U_{e 3}^{*}\left|\nu_{3}\right\rangle
$$

The PMNS matrix, $U$, derived in [4], has the following form:

$$
\begin{aligned}
U= & {\left[\begin{array}{ccc}
U_{e 1} & U_{e 2} & U_{e 3} \\
U_{\mu 1} & U_{\mu 2} & U_{\mu 3} \\
U_{\tau 1} & U_{\tau 2} & U_{\tau 3}
\end{array}\right] } \\
= & {\left[\begin{array}{ccc}
c_{12} c_{13} & s_{12} c_{13} & s_{13} e^{-i \delta} \\
-s_{12} c_{23}-c_{12} s_{13} s_{23} e^{-i \delta} & c_{12} c_{23}-s_{12} s_{13} s_{23} e^{-i \delta} & c_{13} s_{23} \\
s_{12} s_{23}-c_{12} s_{13} c_{23} e^{-i \delta} & -c_{12} s_{23}-s_{12} s_{13} s_{23} e^{-i \delta} & c_{13} c_{23}
\end{array}\right], }
\end{aligned}
$$

where $c_{j k}=\cos \theta_{j k}$ and $s_{j k}=\sin \theta_{j k}$ (subscripts $j$ and $k$ represent two different mass states). In Equation 1.7, $\theta_{j k}$ denotes the mixing angle ${ }^{4}$ and $\delta$ the $\mathrm{CP}$ violating phase. CP violation is the violation of the combined charge conjugation symmetry ${ }^{5}$

${ }^{4}$ The mixing angles indicates how different the flavor eigenstates are from the mass eigenstate.

${ }^{5}$ In particle physics, symmetry exists when certain particle properties (e.g., in coordinates or charge) remain invariant under certain transformations. 
(transforming a particle to its antiparticle by changing its charge) and parity symmetry (transforming one spatial coordinate to another via a change in its sign). It is important as it can tell us why the universe, as it is today, is matter-dominated (as opposed to having an equal amount of matter as antimatter); this is known as matter-antimatter asymmetry. Quarks have been observed to violate CP; however, the question of leptonic CP violation (LCPV) is still open. Neutrino flavor oscillation (explained below) is one of the ways in which LCPV can manifest itself.

The existence of a mixing matrix is an indication that the neutrinos would change flavor as they propagate through space. As highlighted by Equation 1.4, in the three-flavor neutrino mixing, a neutrino with a definite flavor is written as a linear combination of three different mass states. Therefore, when a neutrino is produced at the source ${ }^{6}$ it will arrive to the detector with a different phase,

$$
\left|\nu_{i}(t)\right\rangle=e^{-i\left(E_{i} t\right)}\left|\nu_{i}(0)\right\rangle
$$

where $E_{i}$ is the energy corresponding to the mass eigenstate $i$ and $t$ the time it takes the neutrino to travel from the source to the detector. By the time the neutrino reaches the detector, the mass states have different relative phases than those mass states at the source $\left(\nu_{i}(0)\right.$ in Equation 1.8). Therefore, at the detector, a different flavor state, not present in the beam to begin with, is detected. This is indicated in the relation below (obtained by substituting Equation 1.8 into Equation 1.4):

$$
\left|\nu_{\alpha}(t)\right\rangle=\sum_{i} U_{\alpha i}^{*} e^{-i\left(E_{i} t\right)}\left|\nu_{i}(0)\right\rangle
$$

Namely, a neutrino changes flavor while traveling from a source to a detector because of the different energies of its mass states,

$$
E_{i}=\sqrt{p^{2}+m_{i}^{2}}
$$

${ }^{6}$ Current experiments are unable to resolve the mass states; otherwise, it would have been possible to closely follow each mass state as it propagates from the source to the detector. 
where $p$ is the neutrino momentum. The probability of detecting a different neutrino flavor some distance away from the source is called the oscillation probability; as an example, for a two-flavor oscillation from $\nu_{\mu}$ to $\nu_{e}$, the probability that a $\nu_{\mu}$ at the source changes to a $\nu_{e}$ a distance $L$ from the source is [4]:

$$
\begin{aligned}
P\left(\nu_{\mu} \rightarrow \nu_{e}\right) & =\left|\left\langle\nu_{e}(t) \mid \nu_{\mu}\right\rangle\right|^{2} \\
& =\left|\sum_{i} U_{\mu i}^{*} e^{-i\left(E_{i} t\right)}\left\langle\nu_{e}(t) \mid \nu_{i}(0)\right\rangle\right|^{2} \\
& =\left|\sum_{i} U_{\mu i}^{*} e^{-i\left(E_{i} t\right)} U_{e i}^{*}\left\langle\nu_{1}(t) \mid \nu_{i}(0)\right\rangle\right|^{2} \\
& =\left|\sum_{i} U_{\mu i}^{*} e^{-i\left(p^{2}+\frac{m_{i}^{2}}{2 E}\right) t} U_{e i}^{*}\right|^{2} \\
& =\left|\sum_{i} U_{\mu i}^{*} e^{-i\left(p^{2}+\frac{m_{i}^{2}}{2 E}\right) L / c} U_{e i}^{*}\right|^{2} \\
& =2 \sin ^{2}\left(2 \theta_{13}\right) \sin ^{2}\left(\theta_{23}\right) \sin ^{2}\left(1.27 \Delta m_{32}^{2} \frac{L}{E}\right)
\end{aligned}
$$

where $\theta_{j i}$ is the mixing angle, $\Delta m_{j i}^{2}$, the mass-squared difference, and $E$ the (total) neutrino energy (in the approximation that $m_{i} \ll E_{i} \approx E$, energy associated with mass eigenstate $i, E_{i}$ is equal to $\left.p+\frac{m_{i}^{2}}{2 E}\right)$. For non-zero mixing angle, $\theta_{j i}$, and masssquared difference $\Delta m_{j i}^{2}$, there is a non-zero probability that the $\nu_{e}$ with an energy $E$ will change to a $\nu_{\mu}$ a distance $L$ from the source (Equation 1.11). The mass-squared difference in the case of two-flavor oscillations would be the difference between the two mass states, $\Delta m_{j i}^{2}=m_{j}^{2}-m_{i}^{2}$. For neutrino oscillation to take place, not only must one of the masses be nonzero, it is the nonzero mass-squared value that allows the relative phases between the two mass states to differ. Current experiments can measure the mass-squared difference, but they cannot measure the absolute mass values of each of the mass states - a crucial step in figuring out the sign of $\Delta m_{31}^{2}$ and resolving the neutrino mass hierarchy (NMH) problem (Fig. 1.3). ${ }^{7}$ Currently, there are

${ }^{7}$ The neutrino mass hierarchy is the question of how the mass states are ordered. 


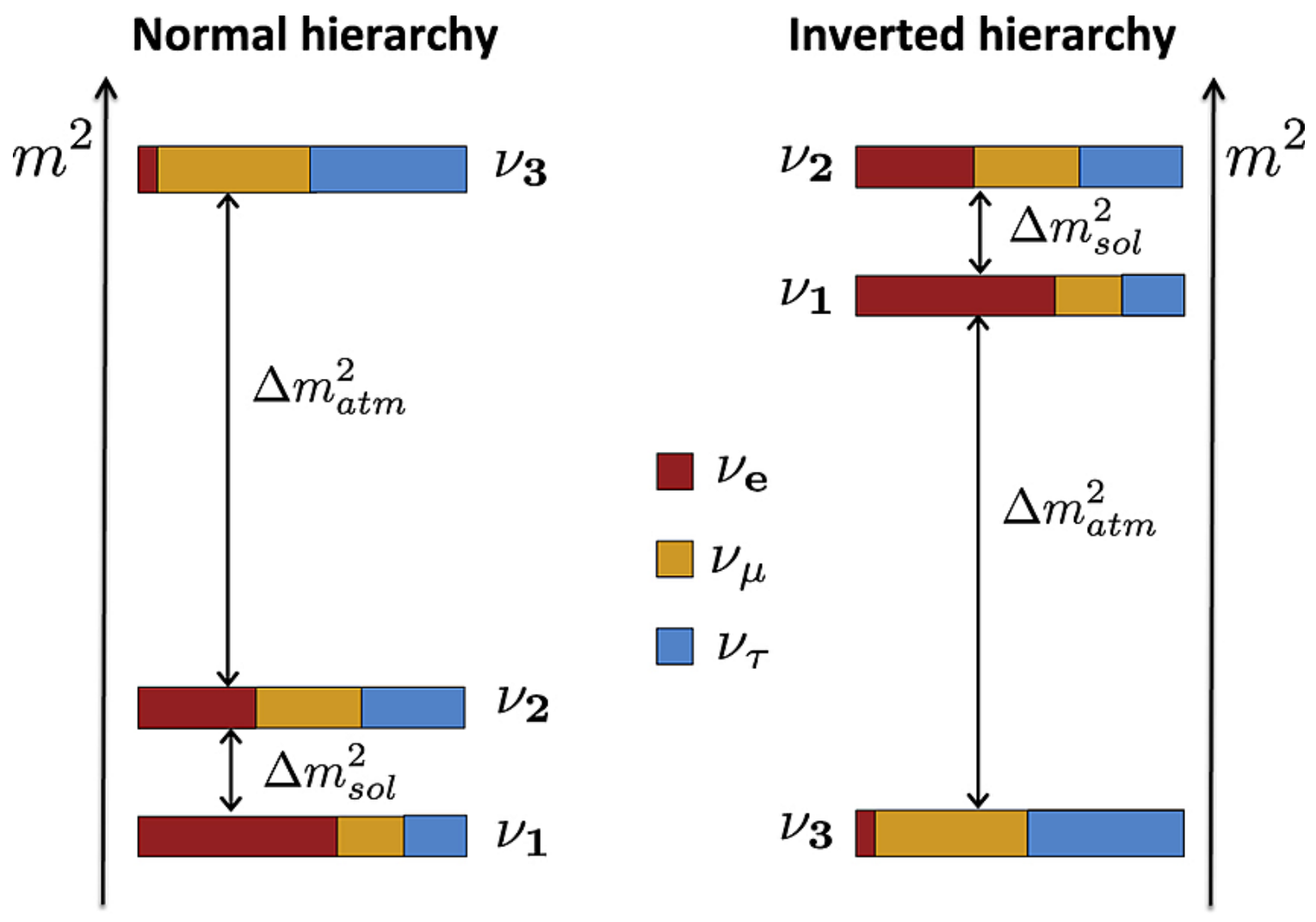

Figure 1.3. Mass hierarchy as illustrated for the two different cases of normal hierarchy (left) and inverted hierarchy (right). The terms $\Delta m_{\text {atm }}^{2} \approx\left|\Delta m_{31}^{2}\right| \approx\left|\Delta m_{32}^{2}\right|$ and $\Delta m_{\text {sol }}^{2} \approx$ $\Delta m_{21}^{2}$ are the atmospheric and solar mass-squared splittings, respectively [10].

two possibly allowed mass orderings, and choosing one over the other has significant theoretical consequences. One mass hierarchy hypothesis is that the mass state $\nu_{1}$ or $m_{1}$ has the largest value while $m_{3}$ has the smallest value. Because this resembles the mass ordering of the charged leptons, it is denoted as the normal hierarchy. In the second hypothesis, known as the inverted hierarchy, $m_{3}$ has the smallest value and $m_{2}$ the largest value (Fig. 1.3). The ultimate goal of the neutrino experiments is to formulate the SM such that the observed neutrino masses and mixing parameters are understood, and the mass hierarchy is a crucial part of that understanding.

Neutrino flavor oscillation was first proposed by Bruno Pontecorvo in 1957, and the first experiment to search for it was the Homestake experiment, located at the Homestake Gold Mine in Lead, South Dakota, and led by Raymond Davis, Jr. 
(1967). Davis [11] placed a tank of perchloroethylene, $\mathrm{C}_{2} \mathrm{Cl}_{4}$ (dry-cleaning solution), $1.5 \mathrm{~km}$ underground to look for the $\nu_{e}$ from the sun (solar neutrino). The $\nu_{e}$ interacted with the chlorine $\left({ }^{37} \mathrm{Cl}\right)$ to produce ${ }^{37} \mathrm{Ar}$ and an electron,

$$
\nu_{e}+{ }_{17}^{37} \mathrm{Cl} \rightarrow{ }_{18}^{37} \mathrm{Ar}+e^{-}
$$

where ${ }^{37} \mathrm{Ar}$ is an unstable isotope that after undergoing electron capture decays back to ${ }^{37} \mathrm{Cl}$. The ${ }^{37} \mathrm{Ar}$ atoms were removed from the solution and counted. From the ${ }^{37} \mathrm{Ar}$ count, the flux of the solar $\nu_{e}$ 's was determined; however, the measured $\nu_{e}$ flux was consistently about $\frac{1}{3}\left(2.5 \times 10^{-36}\right.$ neutrino interactions per target atom per second $)$ of the $\nu_{e}$ flux expected from the solar model $\left(8.1 \times 10^{-36}\right.$ neutrino interactions per target atom per second). This $\nu_{e}$ deficiency, known as the "solar neutrino problem", caused some skepticism among the physicists concerning the validity of the standard solar model. Only after three decades of careful work, the standard solar model and Davis' experimental results were confirmed to be valid. The experiment following the Homestake experiment was the Kamioka Neutrino Detection Experiment II or Kamiokande-II [12] located in Mozumi Mine in Hida's Kamioka area in Japan. It used 50-ktons of water Cherenkov as a detector medium. ${ }^{8}$ The main detection mode was the elastic scattering of electron neutrinos off of the atomic electrons, $\nu_{e}+e \rightarrow \nu_{e}+e$. The electrons resulting from this interaction produced a cone of Cherenkov radiation recorded by the PMTs. The recorded energy and direction of the scattered electrons, as well as the orientation of the Cherenkov ring, was then used to verify that the neutrinos were indeed coming from the sun. In 1989, Kamiokande-II [12] confirmed the same solar $\nu_{e}$ deficiency as the Homestake experiment. The GALLium Experiment or GALLEX [13], at Laboratori Nazionale del Gran Sasso in Gran Sasso, Italy, and the Soviet-American Gallium Experiment, or SAGE [14] at Baksan Neutrino Observatory

${ }^{8}$ Cherenkov detectors are based on the Cherenkov mechanism where particles moving faster than the speed of light in the detector medium emit Cherenkov radiation. 
in the Caucasus mountains in Russia were among the next set of experiments that were able to confirm the same deficiency in the solar $\nu_{e}$ flux. Both GALLEX and SAGE [13, 14] used Gallium as the detection medium to detect the solar neutrinos. The reaction mode of interest was the inverse $\beta$-decay:

$$
\nu_{e}+{ }^{71} G a \rightarrow e^{-}+{ }^{71} G e
$$

where the ${ }^{71} \mathrm{Ge}$ (Equation 1.13) was extracted and its decay rate (half-life of 11.43 days) counted. With each decay corresponding to a $\nu_{e}$ capture, the solar $\nu_{e}$ capture rate was measured to be only $1 / 3$ of the predicted rate, confirming the experimental results of the Homestake and Kamiokande-II experiments. In 2001, the Sudbury Neutrino Observatory, or SNO, experiment located in Creighton Mine near Sudbury, Ontario in Canada came online; it used heavy water $\left(\mathrm{D}_{2} \mathrm{O}\right)$ as detector medium with added salt in a later phase of the experiment. The experiment was designed to measure solar neutrino flux via two interaction modes: charged-current, or CC, interaction: $\nu_{e}+d \rightarrow e^{-}+p+p$ and neutral-current, or NC, interaction: $\nu_{x}+d \rightarrow \nu_{x}+n+p$. It was therefore sensitive to all neutrino flavors and could clearly distinguish between the neutrinos produced in $\mathrm{NC}$ and $\mathrm{CC}$ interactions; this enabled the experiment to measure the ratio of the fluxes of $\nu_{e}$ to $\nu_{x}$. From this measurement, the SNO experiment concluded that the electron neutrinos were changing flavors traveling from the sun to earth [15]. At the same time, the Super-Kamiokande (upgraded KamiokandeII) experiment, in addition to precisely measuring the flux and energy of the solar neutrinos [16], was studying the atmospheric neutrinos. The observation was that the upward-moving (produced on an opposite side of the earth) $\nu_{\mu}$ flux was half that of the downward-moving $\nu_{\mu}$. This could only be explained if the $\nu_{\mu}$ were changing into a different flavor (one that cannot be detected in the experiment) while traveling from one side of the earth to the other [17]. The Super-Kamiokande and SNO results combined confirmed that the solar neutrino problem is due to an underlying neutrino property known as neutrino oscillation and that the standard solar model is correct. 
The neutrino oscillation phenomenon is important because it shows that neutrinos have a non-zero mass, which is in contradiction with the SM assumption that neutrinos are massless. As a result, neutrino oscillation is considered a BSM phenomenon that needs to be studied with high precision; this includes measuring oscillation parameters from Equation 1.11 such as $\theta_{j k}$ and $\Delta m_{j k}^{2}$. The ratio of the distance to the neutrino energy, $\frac{L}{E}$ in Equation 1.11, can be controlled experimentally; for the same $\Delta m_{j k}^{2}$ and $\theta_{j k}$, the location of the detector and the energy spectrum can be adjusted so that the experiment is maximally sensitive to these oscillation parameters. The neutrino factory is a proposed facility capable of delivering neutrino beams of unprecedented intensity and purity, with sub-percent neutrino flux uncertainty [18].

\section{$1.3 \quad$ Neutrino Factory}

Conventionally, neutrinos from accelerator facilities are produced from pion decay $\left(\pi^{-} \rightarrow \mu^{-}+\bar{\nu}_{\mu}\right.$ or $\left.\pi^{+} \rightarrow \mu^{+}+\nu_{\mu}\right)$ via the interaction of a proton beam with a fixed target (e.g., $p+p \rightarrow p+p+\pi^{+}+\pi^{-}$or $p+p \rightarrow p+n+\pi^{+}$). In a neutrino factory $(\mathrm{NF})$, however, the aim is to produce neutrinos via the decay of muons from pion decay (i.e., $\mu^{-} \rightarrow e^{-}+\bar{\nu}_{e}+\nu_{\mu}$ and $\mu^{+} \rightarrow e^{+}+\nu_{e}+\bar{\nu}_{\mu}$ ). This leads to a neutrino beam with $50 \% \bar{\nu}_{e}$ and $50 \% \nu_{\mu}$. In addition, since muon decay can be measured precisely, the energy spectrum and flux of the neutrino beam are known to better than 1\% (compared to the current neutrino flux uncertainties of about 5\% [18]). A schematic diagram of a NF is shown in Figure 1.4. There is a challenge with using muons for producing neutrinos: the pion-decay muons are tertiary particles, forming a diffuse beam (with a large volume). To avoid the high cost of building a largeaperture accelerating structure, the beam size needs to be reduced. Beam cooling is the process of reducing the beam phase-space volume. As a result, the NF front end (Fig. 1.5) has a designated cooling section. The latest NF [1] design consists of a proton driver, target, buncher (forms bunches of charged particles), phase rotation 
section (for reducing the energy spread of the beam), and the cooling section.

At the front end of NF (Fig. 1.5), the "proton driver" can deliver a proton beam power of up to $4 \mathrm{MW}$ (equivalent to $3.3 \times 10^{15}$ protons on target), ${ }^{9}$ kinetic energy up to $15 \mathrm{GeV},{ }^{10}$ and a repetition frequency up to $50 \mathrm{~Hz}$ (how often beam pulses are generated at the source). The proton beam then interacts with a liquid-mercury jet target [19], producing pions. The pions are transported to a solenoid decay channel (a solenoid magnet is used to contain the pions decaying into muons and the pion-decay muons and increase the muon production rate) in which they decay into muons. The muons are collected into bunches up to 3 ns in length using radio-frequency (RF) cavities ${ }^{11}$ in the buncher. Before entering the buncher, unwanted particles (such as protons) are removed in the chicane. ${ }^{12}$ High-energy bunches are decelerated, and low energy bunches accelerated, in the phase rotator to do a phase-energy rotation of the collection of bunches. The pion-decay muons occupy a large phase-space volume, and in order to inject the muons into the accelerating structure efficiently, their phase-space volume needs to be reduced ("cooled"). The cooler section performs the beam cooling. The $\mathrm{R} \& \mathrm{D}$ experiment that has tested a prototype of a cooling section is the Muon Ionization Cooling Experiment, MICE [20]. The cooled beam is transported into a linear accelerator followed by a fixed-field alternating-gradient ring (an accelerating structure with a large aperture, capable of rapidly accelerating the

${ }^{9}$ Beam power is the product of the particle energy with the beam current.

${ }^{10}$ One electron volt $(\mathrm{eV})$ is the kinetic energy of an electron accumulated across a potential difference of 1 volt.

${ }^{11} \mathrm{~A}$ radio-frequency cavity is a metallic cavity used to accelerate and manipulate charged particle beams using electromagnetic waves that resonate inside the cavity. The field oscillates, and once in synch with an incoming charged particle, can accelerate the particle.

${ }^{12} \mathrm{~A}$ chicane is a bent solenoid channel that can be used to transmit part of the beam energy spectrum. 


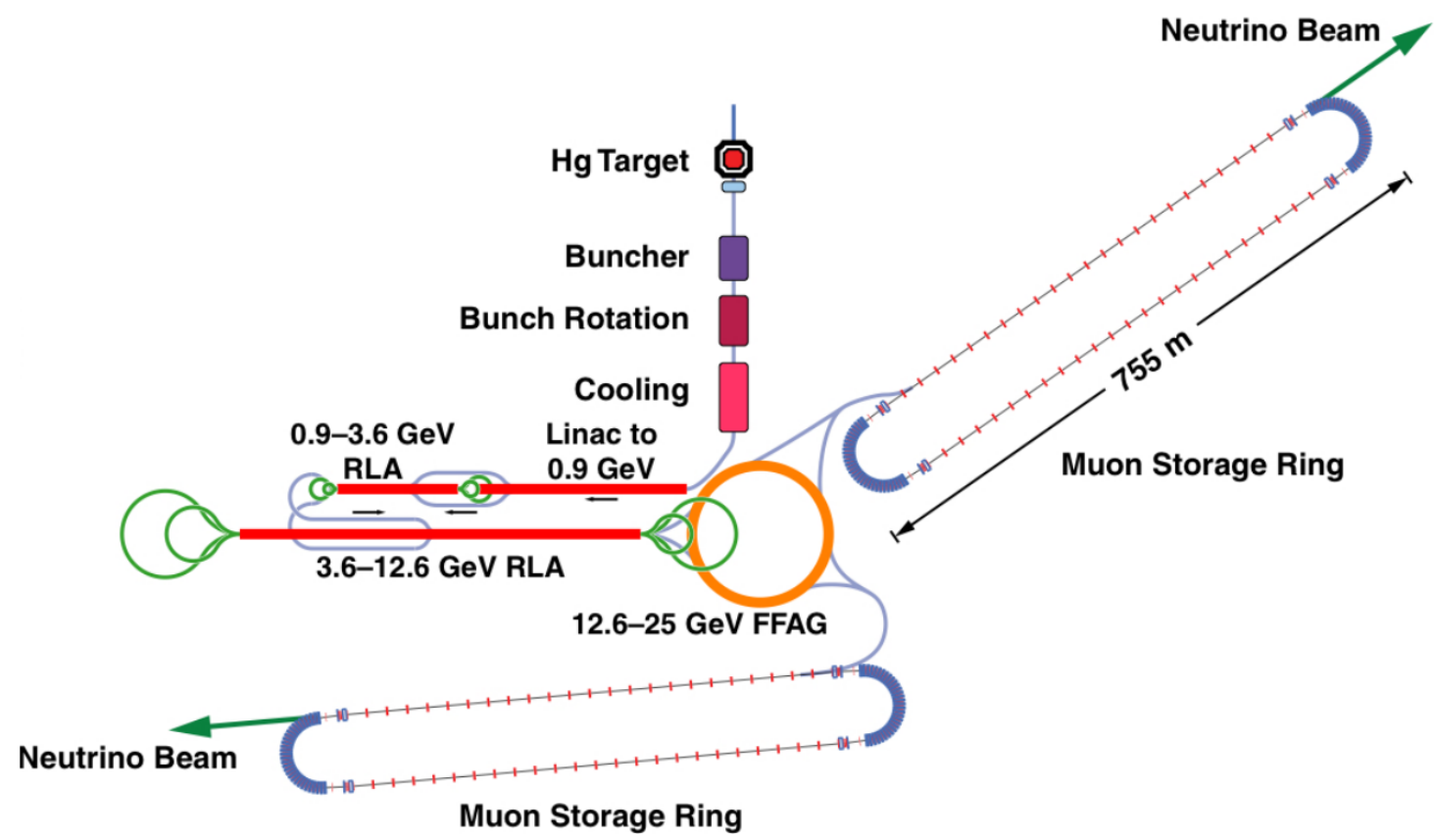

Figure 1.4. Schematic design of a neutrino factory [1].
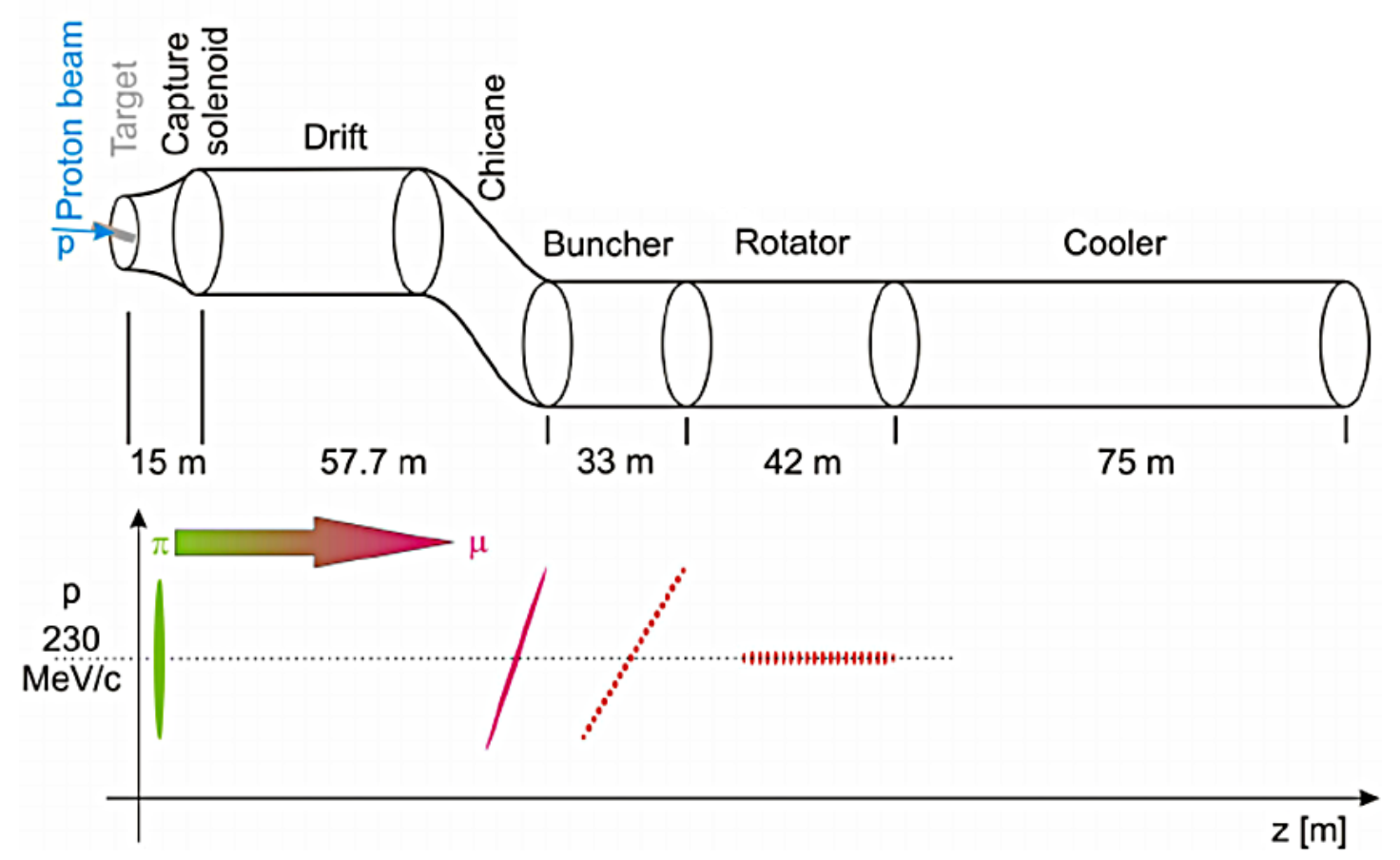

Figure 1.5. Front end of the NF in one of its proposed designs [1]. 


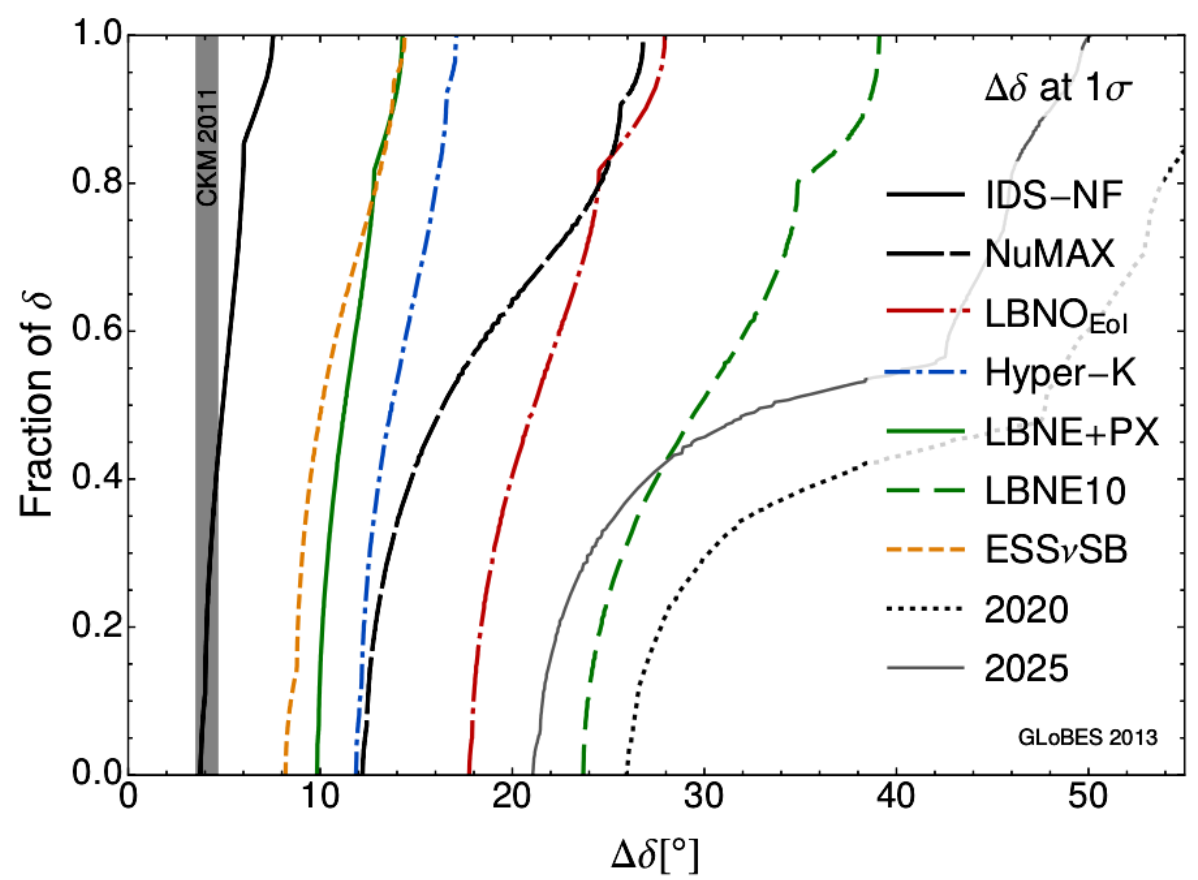

Figure 1.6. Expected precision for the measurement of the CP-violating phase, $\delta$, at various present and future neutrino facilities [23]. The International Design Study for the Neutrino Factory (IDS-NF) demonstrates the best precision for the measurement of the CP-violation.

particles) [21]. The muon storage ring is the ultimate destination where the muons are stored and decay into the desired neutrino beams.

Because of the high-intensity neutrino beam in the NF, the measurement of $\theta_{13}$ using the $\mathrm{NF}$ can reach a precision of $1.5 \%$. In addition, the $\mathrm{CP}$-violating term, $\delta$, and $\Delta m_{31}^{2}$ can be measured to precision of $5^{\circ}$ and $5 \%$, respectively [18, 22] (Fig. 1.6 $6^{13}$ ).

13 "2020" is the combined NOvA and T2K oscillation analysis done by the year 2020 , "ESS $\nu \mathrm{SB}$ " is the $500 \mathrm{kt}$ water Cherenkov detector to be placed at the European Spallation Source, ESS facility in Lund, Sweden, "LBNE10" is the first stage of the Fermilab-based Long Baseline Neutrino Experiment, LBNE is the former name of the current Deep Underground Neutrino Experiment, "LBNE+PX" is the upgrade of the first LBNE stage, "HyperK" is the Hyper-Kamiokande experiment in Japan, "LBNO EoI", the Long Baseline Neutrino Observatory Expression of Interest is a 20 kt Liquid Argon, LAr detector to be placed $2300 \mathrm{~km}$ from CERN, "NuMAX" is the low-luminosity neutrino factory from the decay of $5 \mathrm{GeV}$ muons, and "IDS-NF" is the International Design Study-Neutrino Factory design. 


\subsection{Muon Collider}

In recent years, several LHC successors ${ }^{14}$ capable of surpassing the LHC center of mass collision energy have been proposed. The way a higher energy collision unveils new physics is via two main principles: the first one is the Einstein mass-energy equivalence principle which states that the mass of a particle produced upon collision is proportional to the energies of the colliding particles, with the square of the speed of light as the proportionality constant. Most sought-after elementary particles have large masses (e.g., the top quark, Higgs boson, W and Z bosons, tau lepton, and charm quark), and to produce them, the colliding particles should have enough kinetic energy for conversion into mass upon collision. The second one is Louis de Broglie's principle, which states that a moving particle possesses a simultaneous wave characteristic, and to reduce the particle's wavelength (hence increasing the probing resolution), its momentum (hence its energy) should be increased. These two principles are the main reasons behind the race towards building a next-generation collider facility that can achieve higher center-of-mass energies than the LHC. However, the higher the energies of particles traveling in a collider ring, the harder it is to bend them and keep them in orbit. One solution is to increase the fields in the bending magnets. ${ }^{15}$ The bending radius can be reduced with higher magnetic fields; however, bending magnets capable of achieving such high fields are also more expensive. If the fields are lowered to reduce the magnet cost, the bending radius becomes larger, and the larger the bending radius (hence collider ring circumference), the higher the cost of

${ }^{14}$ The Large Hadron Collider (LHC) is a $27-\mathrm{km}$ circumference collider facility located outside Geneva, Switzerland; it collides beams of protons with the worldrecord center-of-mass energy of $13 \mathrm{TeV}$ and luminosity of $2.06 \times 10^{34} \mathrm{~cm}^{-2} \mathrm{~s}^{-1}$. The biggest LHC milestone was the discovery of the Higgs boson in 2012.

${ }^{15} \mathrm{~A}$ bending or dipole magnet has two iron poles that generate constant magnetic fields and is used to steer a beam of charged particles. 
the collider facility. In addition, the higher the energy of a moving charged particle for the same bending radius, the higher its energy loss due to synchrotron radiation. ${ }^{16}$ Therefore, synchrotron radiation is another limiting factor in achieving higher energies in a collider. In addition to collision energy, luminosity also determines the physics reach of a collider. The definition of instantaneous luminosity, $\mathcal{L}$ starts with the number of events, $N$ and the cross-section, $\sigma$ :

$$
N=\sigma \int \mathcal{L}(t) d t
$$

With bunched beams employed at today's particle colliders, the collider luminosity is defined as,

$$
\mathcal{L}=f \frac{n_{1} n_{2}}{4 \pi \sigma_{x} \sigma_{y}},
$$

where $n_{1}$ and $n_{2}$ characterize the number of particles in each of the two colliding bunches, $\sigma_{x}$ and $\sigma_{y}$ are the root-mean-square (RMS) transverse beam sizes along the horizontal and the vertical directions, respectively, and $f$ is the collision frequency (how often the bunches collide) [9]. Equation 1.15 can be rewritten in terms of the beam emittances (beam phase-space area) $\varepsilon_{x}$ and $\varepsilon_{y}$, and the smallest achievable beta functions (the beam parameter representing the smallest achievable focusing of the bunched beam at the interaction point), $\beta_{x}^{*}$ and $\beta_{y}^{*}$ [9],

$$
\mathcal{L}=f \frac{n_{1} n_{2}}{4 \sqrt{\varepsilon_{x} \beta_{x}^{*} \varepsilon_{y} \beta_{y}^{*}}} .
$$

Equation 1.16 is used in assessing the performance of a future collider facility. Figure 1.7 demonstrates the luminosity reach of some of the future LHC successors.

Among the proposed future collider facilities are those that are aimed at accelerating and colliding leptons, such as the future circular collider FCC-ee and muon

${ }^{16}$ Charged particles accelerating in a ring emit a form of electromagnetic radiation known as synchrotron radiation. Synchrotron radiation is a crucial obstacle faced when designing a next-generation high-energy collider. The energy loss due to synchrotron radiation scales as the fourth power of the accelerating particle's energymass ratio. 


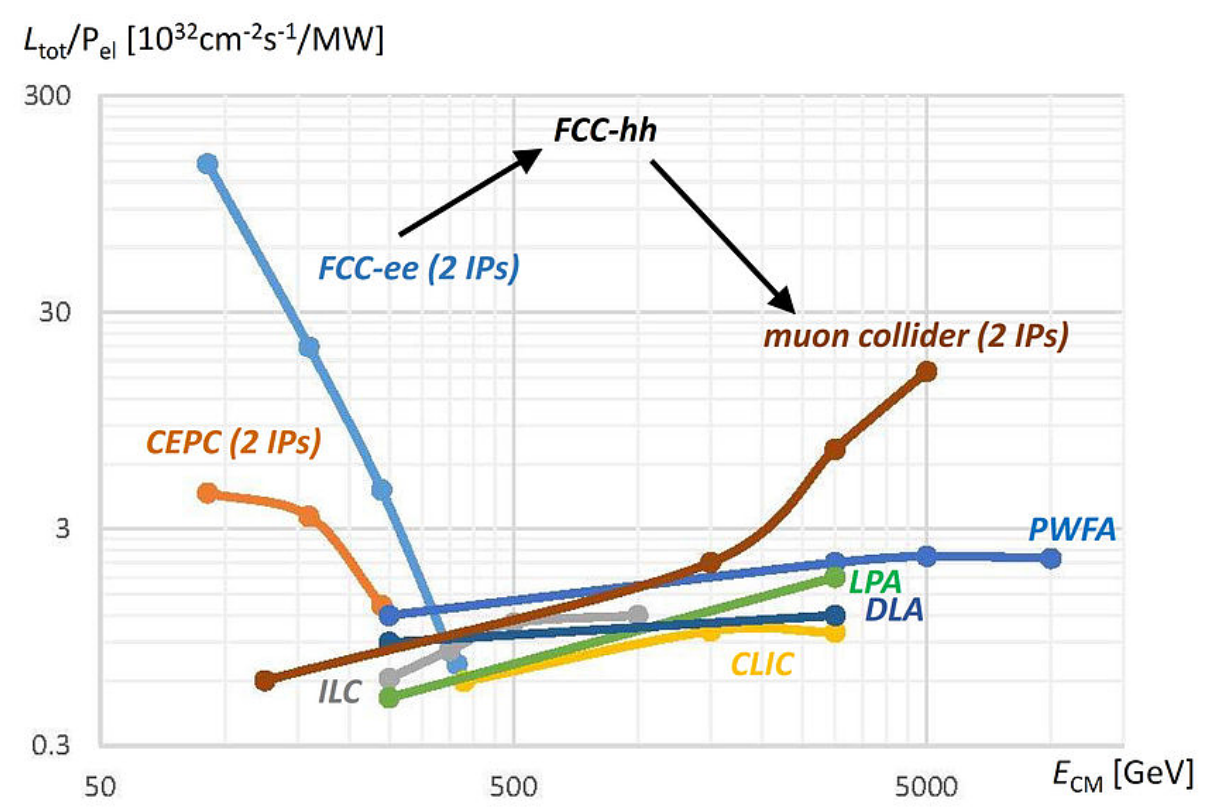

Figure 1.7. Luminosity per electrical power (power consumed by the collider facility) versus center of mass energy for various proposed collider facilities [24, 25].

collider (also referred to as $\mu^{+} \mu^{-}$collider). The advantage of a lepton over a hadron (a composite particle such as a proton) is that all of the center-of-mass energy is available for particle production during the collision. This means more physics information can be extracted from the collision (e.g., $\mu^{+} \mu^{-} \rightarrow H$ or $e^{+} e^{-} \rightarrow Z H$ ). On the other hand, muons are about 207 times heavier than the electrons and thus lose $10^{9}$ times less energy through synchrotron radiation than electrons of the same beam energy. For this reason, a $\mu^{+} \mu^{-}$collider can reach multi-TeV energies with a substantially smaller ring than an $e^{+} e^{-}$circular collider (Fig. 1.7). The energy loss through synchrotron radiation, $U$, per turn in a circular collider depends strongly on the bending radius, $\rho$, and the velocity of the moving charged particle, $\beta c$ [9],

$$
U=\frac{C_{\gamma} \beta^{3} E_{0}^{4}}{\rho}
$$

where $C_{\gamma}=\frac{4 \pi r_{0}}{3\left(m c^{2}\right)^{3}}\left(r_{0}\right.$ is the classical radius of electron and is equal to $\left.\frac{e^{2}}{4 \pi \epsilon_{0} m c^{2}}\right)$ for muon is $4.84 \times 10^{-14} \frac{\mathrm{m}}{(\mathrm{GeV} / c)^{3}}$ and $E_{0}$ is the nominal energy of the moving charged particle [26]. In addition, because of the muon large mass, the direct "s-channel" 


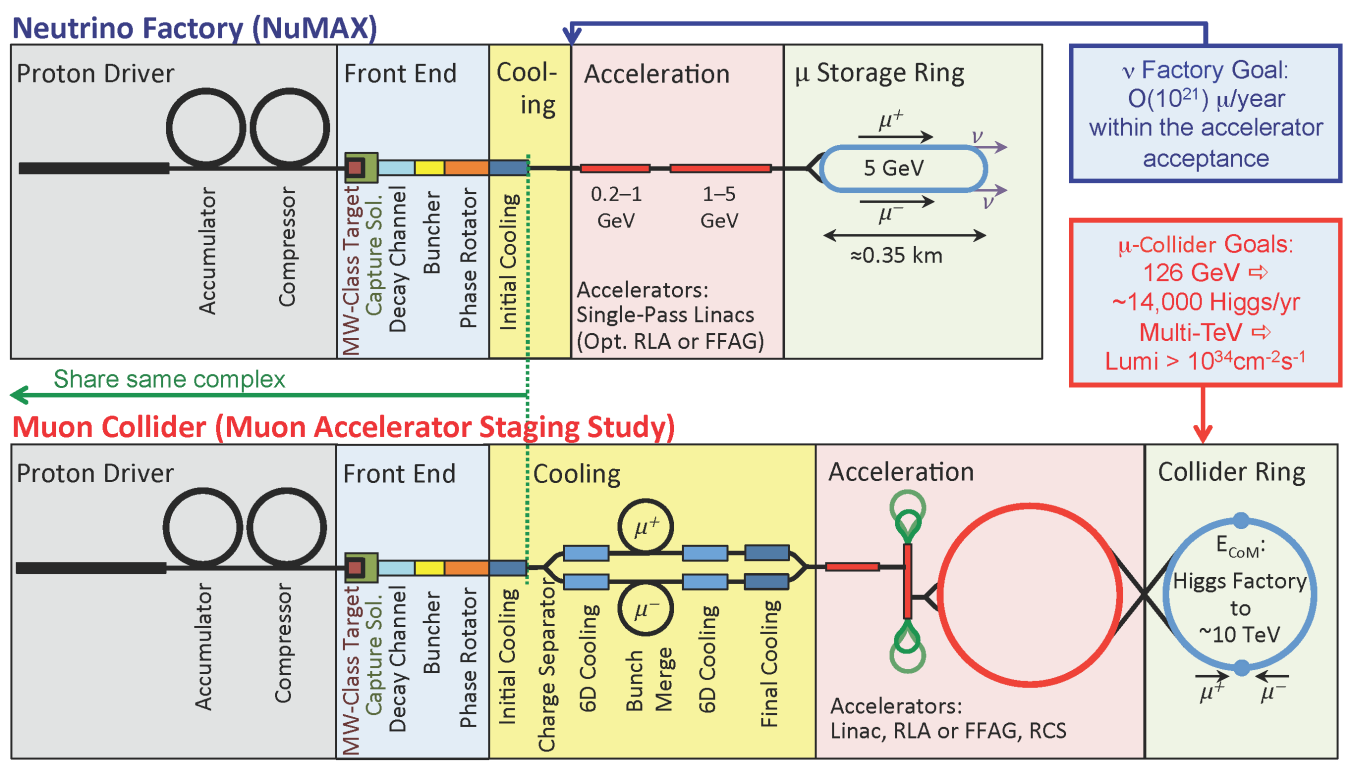

Figure 1.8. Muon collider and neutrino factory schematic designs compared. The two facilities can share the same complex and be built on the same experimental site [27].

Higgs-production rate is enhanced by a factor of about 40000 compared to the rate in an $e^{+} e^{-}$machine, making it possible to scan the center-of-mass energy to measure the Higgs-boson line shape directly [18]. Schematic diagrams of a muon collider and a neutrino factory are shown in Figure 1.8. The muon collider and neutrino factory share the same front end and, hence, can share the same complex and be built on the same experimental site.

The muon production in a muon collider starts with the interaction of the proton beam with a mercury target. The pions from this interaction are captured inside a solenoid magnet known as a capture solenoid. The buncher and phase rotator perform further beam "conditioning" and reduce the energy spread. The muons then reach the ionization cooling channel. Because muons are tertiary particles (protons interact with the target, producing pions that decay into muons), they form a beam of large phase-space volume. To fit more muons into smaller cost-effective accelerating apertures, the volume of the muon beam needs to be reduced. The process of 
reducing the beam phase-space ${ }^{17}$ volume is called beam cooling. The beam cooling techniques such as electron cooling and stochastic cooling, ${ }^{18}$ however, are not fast enough for muons because of the short muon lifetime. The only technique that applies to muons is ionization cooling [28], a beam cooling technique that reduces muon beam phase-space volume via ionization energy loss of muons in the absorbing material. The experiment that has demonstrated this technique is the Muon Ionization Cooling Experiment (MICE). MICE employs a single cooling cell (containing one absorber). For full six-dimensional cooling with re-acceleration, a typical ionization cooling channel in a muon collider will have a few hundred cooling cells [29] (because a muon collider requires $\mathrm{O}\left(10^{6}\right)$ reduction in six-dimensional emittance), with each cooling cell consisting of an absorbing material sandwiched between RF cavities. The ionization cooling channel is an indispensable component of a future muon collider, and demonstrating muon ionization cooling is thus an essential R\&D step towards building both a future muon collider and neutrino factory.

In addition to muon production via pion decay, which starts from the interaction of proton beam with a target, it is possible to form a naturally cooled muon beam via $\mu^{+} \mu^{-}$pairs created in electron-positron annihilation. The Low Emittance Muon Accelerator (LEMMA) program is the R\&D effort behind this alternative muon production scheme $[18,30]$. The idea is to collide a positron beam with energies just above the $\mu^{+} \mu^{-}$production threshold with a stationary thin target. The collision causes the positrons to annihilate with the atomic electrons of the target and as a

${ }^{17} \mathrm{~A}$ charged particle in a beam is characterized by three spatial and three momentum coordinates, forming an overall six-dimensional phase space. These coordinates are referred to as phase-space coordinates.

${ }^{18}$ In electron cooling, electrons are injected into a charged particle beam, causing the beam to interact with the electrons coulombically and lose momentum. In stochastic cooling, a feedback system sends electrical signals to the charged particle beam to correct its angular and energy spread with respect to the design orbit [31]. 
result, produce muon-antimuon pairs $\left(e^{+} e^{-} \rightarrow \mu^{+} \mu^{-}\right)$. The advantage of this production scheme is that the muons form low emittance beams upon production (hence rendering muon beam cooling at the front-end of the collider facility). The challenge with using a muon collider scheme such as LEMMA is in the required muon rate and luminosity $[18,30]$. MICE is the required R\&D effort for a proton-on-target piondecay muon collider scheme; however, a muon collider scheme based on LEMMA may still require muon cooling for achieving the desired luminosity. 


\section{CHAPTER 2 \\ MUON IONIZATION COOLING EXPERIMENT}

\subsection{The Principle}

One way of producing muons is to collide high-power protons with a target to produce pions that then decay into muons. The muon beam formed this way has a large phase-space volume. One way of measuring the beam phase-space volume is to calculate a quantity known as beam emittance. Typical transverse ${ }^{19}$ emittance $^{-1}$ of a highly diffuse muon beam has a range between $15 \pi$ and $20 \pi \mathrm{mm} \cdot \mathrm{rad}[32,33]$ and the initial longitudinal emittance is typically around $170 \pi \mathrm{mm} \cdot \mathrm{rad}$. The desired muon beam transverse emittance in the neutrino factory has a range between $2 \pi$ and $5 \pi \mathrm{mm} \cdot \mathrm{rad}$. A muon collider requires further cooling with a desired transverse emittance of $0.025 \pi \mathrm{mm} \cdot \mathrm{rad}$, and longitudinal ${ }^{20}$ emittance of $72 \pi \mathrm{mm} \cdot \mathrm{rad}$ [33] with overall six-dimensional (6D) emittance reduction of $\mathrm{O}\left(10^{6}\right)$. Because of the muon's large mass and short lifetime, conventional cooling techniques such as synchrotron radiation damping, ${ }^{21}$, and stochastic cooling cannot be used for a beam of muons. Ionization cooling is the only beam cooling technique suitable for reducing the muon beam emittance within the short muon lifetime [28]. The international Muon Ionization Cooling Experiment, MICE (Figs. 2.1 and 2.2), is the first experiment that

\footnotetext{
${ }^{19}$ Transverse coordinates are along the plane perpendicular to the direction of motion.

${ }^{20}$ Longitudinal coordinates are along the direction of motion. The transverse and longitudinal phase-space coordinates are the locations and momenta of the particles that deviate from the design trajectory.

${ }^{21}$ Synchrotron radiation damping is the process of reducing the beam phasespace volume through synchrotron radiation energy loss.
} 


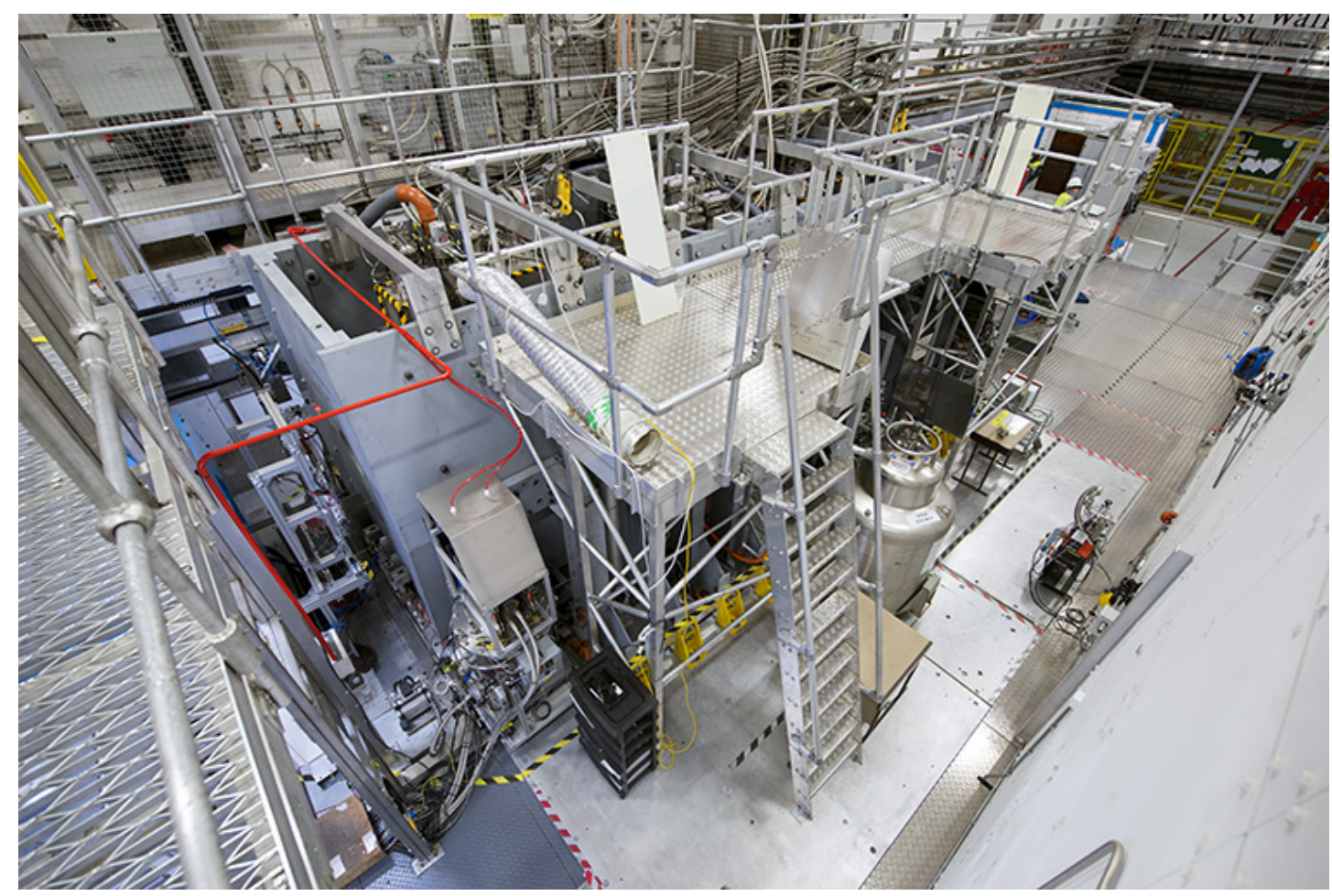

Figure 2.1. A 2016 photo of the Muon Ionization Cooling Experiment, MICE, in its final configuration showing partial return yoke surrounding the upstream and downstream Spectrometer Solenoid and Absorber Focus Coil modules.

has demonstrated this technique for muons. Ionization cooling in MICE occurs when muons lose momentum via their interactions with the atomic electrons of the absorbing material. There are two scenarios under which muons can lose energy as they traverse matter: they can undergo a so-called "soft" inelastic collision, where the collision impact parameter is much larger than the atomic Bohr radius. Under such conditions, the atom goes into an excited energy state as it acquires energy from the muon [9]. In addition, the muons can undergo a so-called "knock-on" collision; this happens when the incoming muon energy is significantly larger than the electron binding energy. In such conditions, the muon causes the atom to ionize via ejection of a valence electron; this is the ionization energy loss process [9]. The Bethe-Bloch 


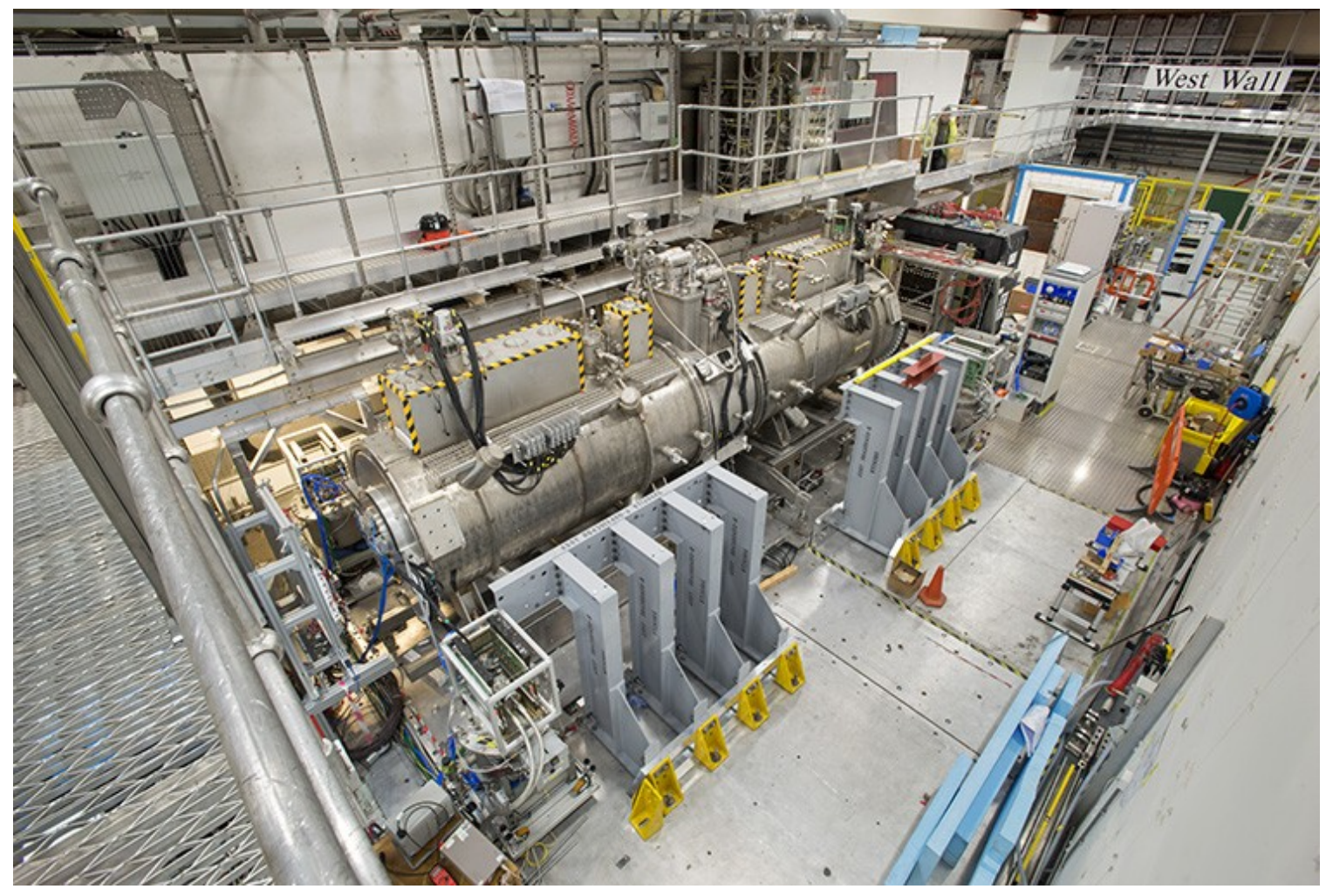

Figure 2.2. A 2015 photo of the Muon Ionization Cooling Experiment, MICE, in its final configuration prior to the installation of the partial return yoke, showing the upstream and downstream Spectrometer Solenoid and the Absorber Focus Coil modules. 
formula [9] is used in parametrizing ionization energy loss in material,

$$
\frac{d E}{d x} \approx \frac{Z}{A}\left(0.307 \mathrm{MeV} \mathrm{cm}^{2} / \mathrm{g}\right) \frac{q^{2}}{\beta^{2}}\left[\frac{1}{2} \ln \left(\frac{2 m_{e} c^{2} \beta^{2} \gamma^{2} T_{\max }}{I^{2}}\right)\right],
$$

where $A$ is the atomic mass number and $Z$ atomic number of the absorbing material, $q$ the charge of the incident particle, $I$ the characteristic material-dependent ionization constant, $T_{\max }$ the maximum energy transfer [9], $\beta c$ the velocity of the incident charged particle, $m_{e}$ the mass of the electron, and $\gamma$ the relativistic Lorentz term, $\frac{1}{\sqrt{1-\beta^{2}}}$. The Bethe-Bloch relation describes the average rate of energy loss of charged particles in material as a function of initial particle momentum. The reference momentum for cooling in MICE is around $200 \mathrm{MeV} / c$, which is near the minimum of the ionization energy-loss in the $d E / d x$ curve. The reason is mainly to find a compromise between the heating effects of the "straggling tail" (further details below) at higher momentum and the negative slope of the $d E / d x$ curve below the ionization minimum. Figure 2.3 [28] shows the rates of muon energy loss in various materials. The energy loss decreases rapidly as the particle momentum increases and reaches a minimum value for muons with momentum around $0.3 \mathrm{GeV} / c$ (minimum ionizing). Above this momentum value, muons enter the region of relativistic rise, where the energy loss increases more slowly [9].

In addition to the ionization energy loss process, elastic collisions with the atomic nuclei can occur. Given the larger mass of the atomic nucleus compared with the incident muon, this process causes very small energy loss. However, it is the driving force behind the scattering of muons at small angles [9]. This process, referred to as multiple Coulomb scattering, changes the muon trajectory multiple times as it traverses material, and as a result, the muon exiting the material forms an angle $\theta_{0}$ with the initial trajectory (Fig. 2.4 [9]).

There are generally two ways of describing the motion of a charged particle 


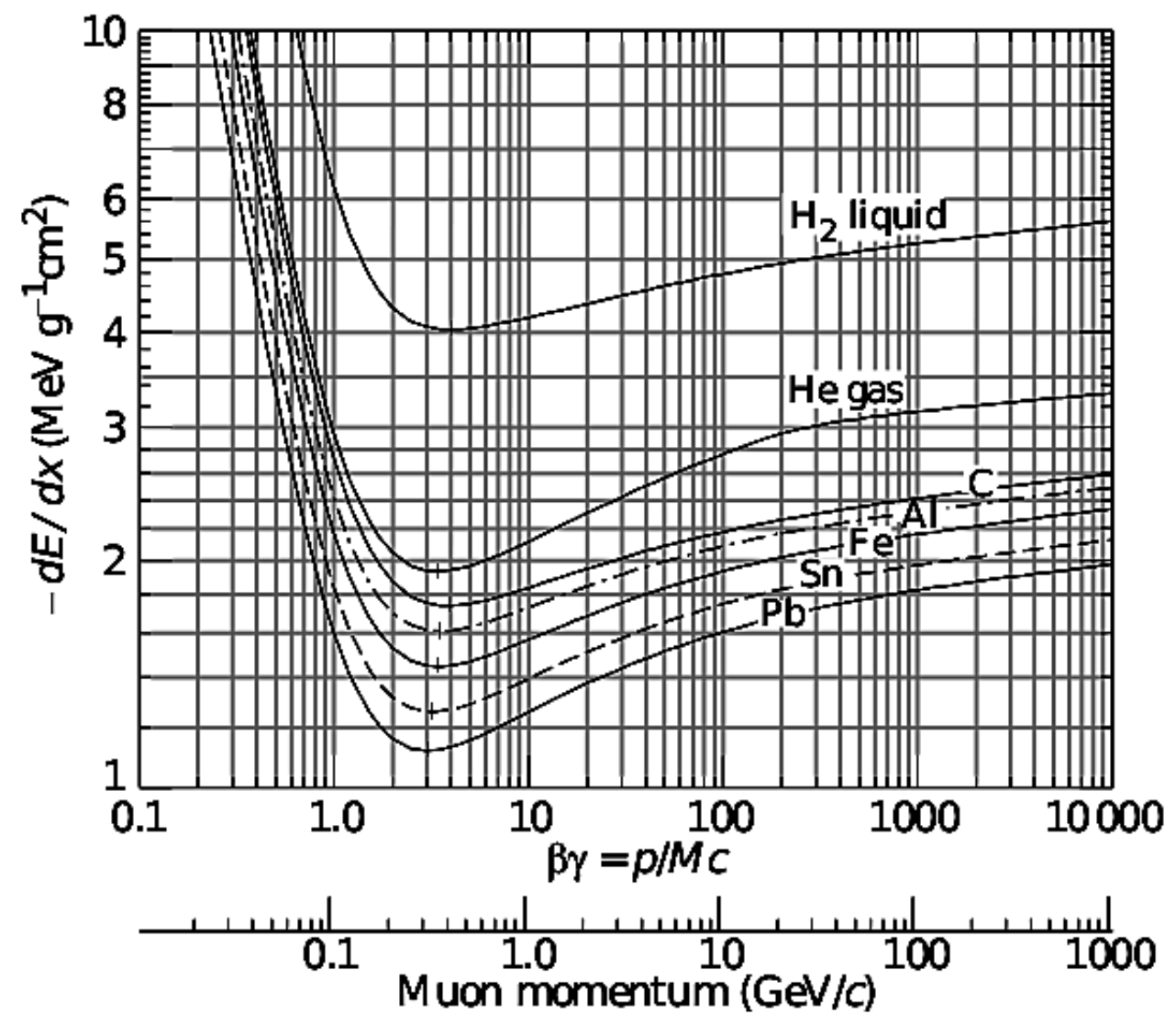

Figure 2.3. Ionization energy loss rate vs. the muon momentum [9].

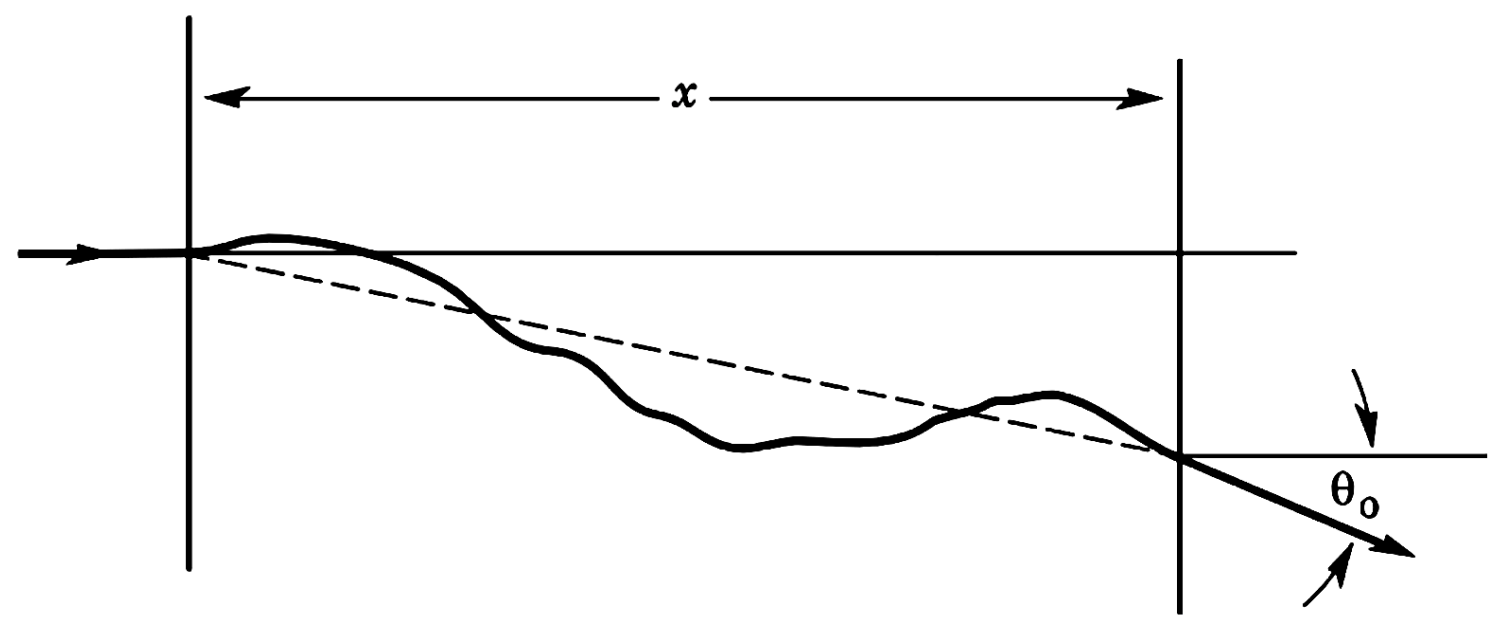

Figure 2.4. Effect of multiple scattering on the particle track as it travels a distance $x$ through material [9]. 
in an accelerating structure or a storage ring: ${ }^{22}$ collectively, using beam envelope equations or individually, using a single-particle approach. In addition, two types of trajectories exist: either a design (also known as a reference) trajectory as shown in Figure 2.5 or a trajectory that deviates from the design trajectory. Since the motion along the reference trajectory is well-understood, what follows below focuses on explaining the motion of the "non-reference" trajectory.

In a single-particle approach, the motion of a beam of charged particles is typically described using the phase-space coordinates $\left(x, p_{x}, y, p_{y}, t, E\right)$ (where $t$ is the time of flight relative to that of the reference particle, $E$ the energy relative to that of the reference particle, and $p_{x(y)}$ the momentum coordinate in the $x(y)$ direction) or the trace-space coordinates $\left(x, x^{\prime}, y, y^{\prime}, t, E\right)$ (where $x^{\prime}=\frac{p_{x}}{p_{0}}, y^{\prime}=\frac{p_{y}}{p_{0}}$, and $p_{0}$ is the reference particle momentum) of each particle. In a collective description, the Twiss parameters, emittance, $\varepsilon$, beta function, $\beta$, alpha, $\alpha$, and gamma, $\gamma$ (described in more detail below) are often used. Since a charged particle in an accelerating structure moves in the presence of electric or magnetic fields, the first step in describing its motion is to derive expressions for the magnetic and electric fields using Maxwell's equations. The next step is to substitute the field expressions into the Lorentz force equation, $\vec{F}=q(\vec{E}+\vec{v} \times \vec{B})$ (where $q$ is the particle's electric charge, $\vec{E}$ the electric field, $\vec{v}$ the velocity of the moving particle, and $\vec{B}$ the magnetic field), from which the equations of motion can be derived. For a magnetic element controlling the transverse motion of a particle in the paraxial approximation, the equations of motion in the transverse direction should follow a Hamiltonian and would be similar to the motion of a harmonic oscillator; there is a restoring force that is proportional to the magnet

${ }^{22}$ The purpose of a storage ring is to store and maintain a particle beam in a ring for several hours. 


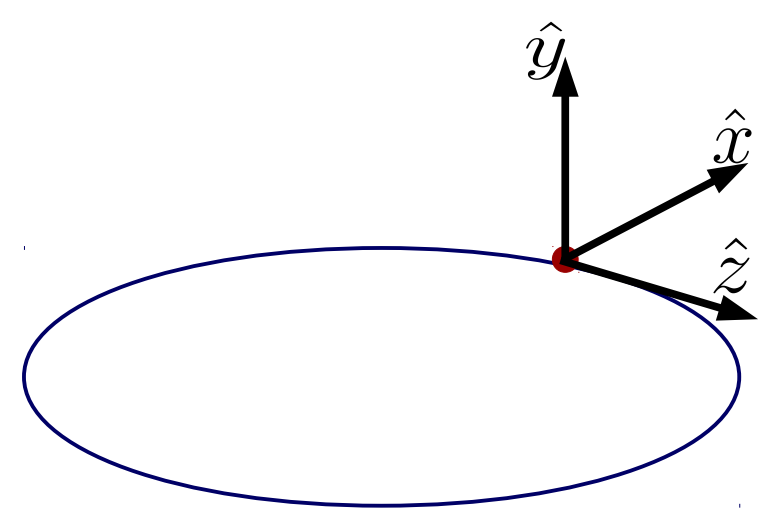

Figure 2.5. An illustration of the motion of the reference particle in an accelerator ring.

strength, $K(z)[26]$,

$$
\begin{aligned}
& x^{\prime \prime} \propto-K(z) x, \\
& y^{\prime \prime} \propto-K(z) y,
\end{aligned}
$$

where $x^{\prime \prime}$ and $y^{\prime \prime}$ are the second derivatives of $x$ and $y$ with respect to the coordinate $z$ (the coordinate describing the position of the particle along the direction of motion as shown in Figure 2.5). While both electric and magnetic fields can be used to control the particle's transverse motion, only electric fields can be used for controlling the particle's longitudinal motion. For this purpose, a Radio Frequency (RF) cavity can be used. As the name suggests, the idea is to excite an electromagnetic mode that oscillates in time inside a cavity, allowing the electric field to be purely along the $z$ direction and the magnetic field along the angular direction $(\theta$ in the cylindrical coordinate system). MICE in its final configuration did not have an RF cavity. For this reason, the longitudinal motion in this thesis is described using the energy coordinate, and the equations of motion in the longitudinal direction are not covered in this thesis.

The relation described by Equation 2.2 is known as Hill's equation. Given a set of initial conditions $x(0)=x_{0}, y(0)=y_{0}, x^{\prime}(0)=x_{0}^{\prime}, y^{\prime}(0)=y_{0}^{\prime}$, and assuming a constant $K$ factor, its solution would be sinosoidal if $K<0$, hyperbolic (hyperbolic sine and cosine functions) if $K>0$, and linear if $K=0$. Using matrix formalism, 
the sinosoidal solution can be written as [26],

$$
\begin{aligned}
R(z) & =M(z \mid 0) R(0) \\
\text { where } M(z \mid 0) & =\left(\begin{array}{cc}
\cos \sqrt{K} z & \sin \frac{\sqrt{K} z}{\sqrt{K}} \\
-\sqrt{K} \sin \sqrt{K} z & \cos \sqrt{K} z
\end{array}\right) \\
\text { and } R(z) & =\left(\begin{array}{c}
x \\
x^{\prime}
\end{array}\right) \\
\text { or } R(z) & =\left(\begin{array}{c}
y \\
y^{\prime}
\end{array}\right) .
\end{aligned}
$$

The matrix $M(z \mid 0)$ is known as the transfer matrix and can be used to determine the transverse coordinates of a moving particle at any point along the accelerator as the particle passes through a magnetic element. ${ }^{23}$

What has been described thus far is the single--particle approach to describing the motion of a charged particle beam. As mentioned above, it is also possible to use a collective approach and study the evolution of a set of lattice-dependent parameters. In this context, the solution to Hill's equation can be written using the CourantSnyder formalism [34],

$$
r(z)=\sqrt{\varepsilon \beta(z)} \cos \left(\psi(z)+\psi_{0}\right)
$$

where $r(z)$ is either $x$ or $y, \beta$ (the beta function) the beam amplitude in the $x$ or $y$ direction, $\varepsilon$ (the emittance) the beam size, and $\psi=\int_{0}^{z} \frac{d z}{\beta(z)}$ the phase advance (related to the focusing strength). A full collective description of the beam can be done using

${ }^{23}$ In the case of a particle drifting a distance $z$ in a region with no magnet, the transfer matrix is $M(z \mid 0)=\left(\begin{array}{ll}1 & z \\ 0 & 1\end{array}\right)$. 
two other parameters: beam alpha, $\alpha(z)=-\frac{1}{2} \beta^{\prime}$ and gamma function or $\gamma(z)=$ $\left(1+\alpha(z)^{2}\right) / \beta(z)$, which represents the beam amplitude in the $x^{\prime}$ or $y^{\prime}$ direction. These parameters are referred to as the Courant-Snyder functions or Twiss parameters and once the $\beta$ function is determined, all the other Twiss parameters can be calculated. Transforming the transverse coordinates to these new parameters in Equation 2.4 can be useful, because unlike MICE, not all accelerator-based experiments have the capability of recording the transverse coordinates of each particle.

One can combine Equation 2.4 with its derivative [26],

$$
r^{\prime}=-\sqrt{\varepsilon} \frac{\alpha}{\sqrt{\beta}} \cos \left(\psi+\psi_{0}\right)-\sqrt{\frac{\varepsilon}{\beta}} \sin \left(\psi+\psi_{0}\right)
$$

to derive the relation [34]

$$
\gamma r^{2}+2 \alpha r r^{\prime}+\beta r^{2}=\varepsilon
$$

which is the equation of an ellipse in the $\left(r, r^{\prime}\right)$ trace space with the area $\pi \varepsilon$. As the particle beam evolves in the accelerator, its shape changes but its area remains constant. A two-dimensional beam ellipse in the $\left(x, x^{\prime}\right)$ trace space is shown in Figure 2.6; the beam ellipse area, tilt, and orientation are determined using the Twiss parameters $[26,34,35]$. For a given distribution of particles in trace space, these parameters could be related to the second moments of the distribution:

$$
\begin{gathered}
\sigma_{x x}=\left\langle x^{2}\right\rangle=\frac{\sum_{i=1}^{n} x_{i}^{2}}{n}, \\
\sigma_{x^{\prime} x^{\prime}}=\left\langle x^{\prime 2}\right\rangle=\frac{\sum_{i=1}^{n} x_{i}^{\prime 2}}{n}, \\
\sigma_{x x^{\prime}}=\sigma_{x^{\prime} x}=\left\langle x x^{\prime}\right\rangle=\frac{\sum_{i=1}^{n} x_{i} x_{i}^{\prime}}{n},
\end{gathered}
$$

where $i$ denotes the $i^{\text {th }}$ particle in the beam distribution, and $n$ is the total number of particles in the beam. The Twiss parameters, expressed in terms of the second moments, are

$$
\varepsilon_{r m s}=\pi \sqrt{\left\langle x^{2}\right\rangle\left\langle x^{\prime 2}\right\rangle-\left\langle x x^{\prime}\right\rangle^{2}}
$$




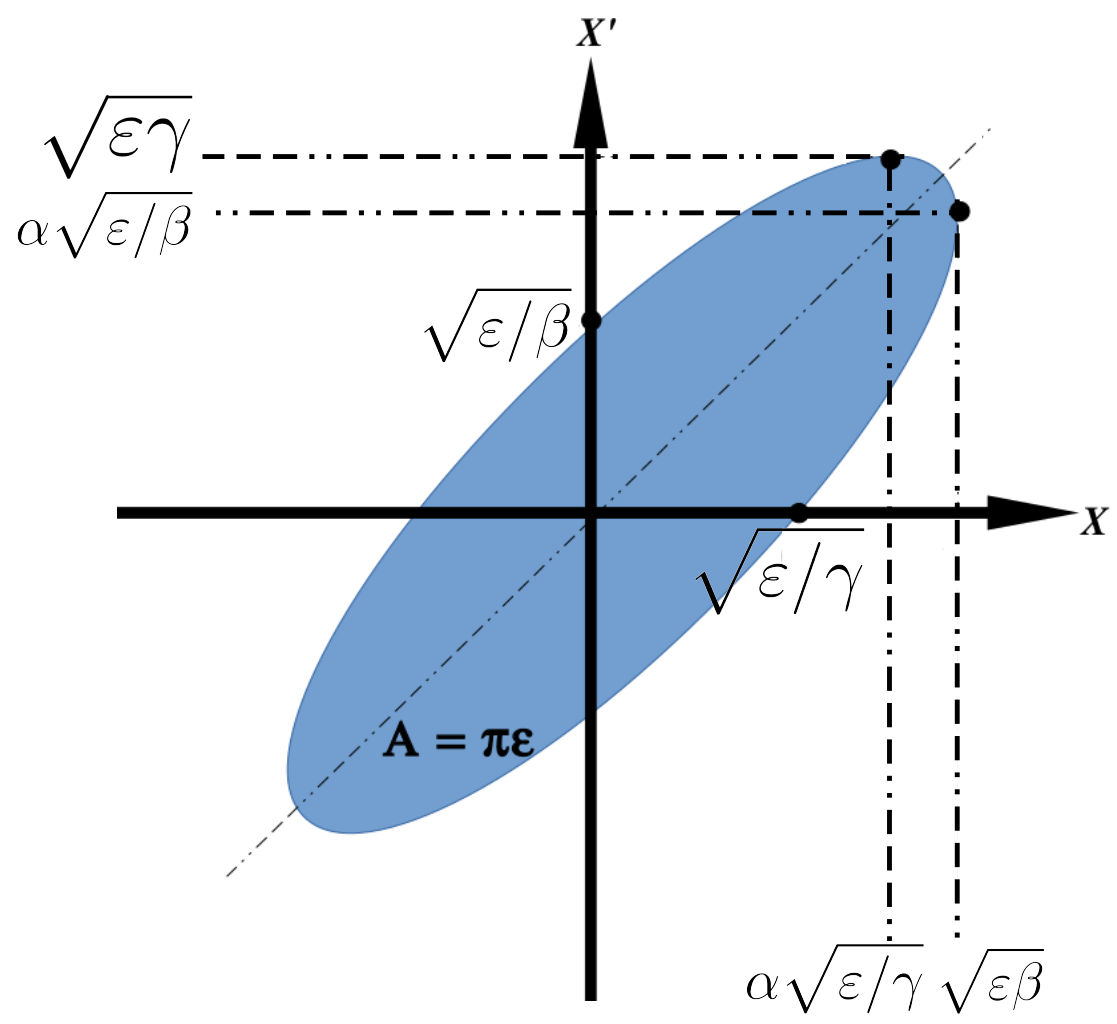

Figure 2.6. The 2D trace space ellipse. The parameters of this ellipse are the Twiss parameters [35].

$$
\begin{gathered}
\beta=\frac{\left\langle x^{2}\right\rangle}{\varepsilon / \pi}, \\
\alpha=-\frac{\left\langle x x^{\prime}\right\rangle}{\varepsilon / \pi}, \\
\gamma=\frac{\left\langle x^{\prime 2}\right\rangle}{\varepsilon / \pi},
\end{gathered}
$$

where $\varepsilon_{r m s}$ is the root-mean-square (RMS) or geometric emittance. Equations 2.7 through 2.13 can be rewritten in the matrix form

$$
\Sigma_{2 \times 2}=\left(\begin{array}{cc}
\sigma_{x x} & \sigma_{x^{\prime} x} \\
\sigma_{x x^{\prime}} & \sigma_{x^{\prime} x^{\prime}}
\end{array}\right)=\left(\begin{array}{cc}
\varepsilon \beta & -\varepsilon \alpha \\
-\varepsilon \alpha & \varepsilon \gamma
\end{array}\right),
$$

where $\Sigma_{2 \times 2}$ is the $2 \mathrm{D}$ covariance matrix.

The square of the determinant of the covariance matrix from Equation 2.14 
divided by the muon mass, $m_{\mu}$, is the expression for the normalized emittance [35]:

$$
\varepsilon_{n}=\beta \gamma \varepsilon_{r m s}=\frac{p_{\text {ref }}}{m_{\mu}}\left|\Sigma_{2 \times 2}\right|^{1 / 2} .
$$

where $p_{\text {ref }}$ is the momentum of the reference particle. Normalized emittance is important because it is conserved, while geometric emittance shrinks under acceleration [35].

In MICE, due to the presence of solenoidal fields, all transverse trace-space coordinates $\left(x, x^{\prime}, y, y^{\prime}\right)$ (or phase-space coordinates) are coupled, and as a result, it is convenient to calculate the four-dimensional transverse emittance. The $4 \times 4$ covariance matrix is

$$
\Sigma_{4 \times 4}=\left(\begin{array}{cccc}
\sigma_{x x} & \sigma_{x^{\prime} x} & \sigma_{y x} & \sigma_{y^{\prime} x} \\
\sigma_{x x^{\prime}} & \sigma_{x^{\prime} x^{\prime}} & \sigma_{y x^{\prime}} & \sigma_{y^{\prime} x^{\prime}} \\
\sigma_{x y} & \sigma_{x^{\prime} y} & \sigma_{y y} & \sigma_{y^{\prime} y} \\
\sigma_{x y^{\prime}} & \sigma_{x^{\prime} y^{\prime}} & \sigma_{y y^{\prime}} & \sigma_{y^{\prime} y^{\prime}}
\end{array}\right),
$$

and for reference, the $6 \times 6$ covariance matrix is

$$
\Sigma_{6 \times 6}=\left(\begin{array}{cccccc}
\sigma_{x x} & \sigma_{x^{\prime} x} & \sigma_{y x} & \sigma_{y^{\prime} x} & \sigma_{t x} & \sigma_{E x} \\
\sigma_{x x^{\prime}} & \sigma_{x^{\prime} x^{\prime}} & \sigma_{y x^{\prime}} & \sigma_{y^{\prime} x^{\prime}} & \sigma_{t x^{\prime}} & \sigma_{E x^{\prime}} \\
\sigma_{x y} & \sigma_{p_{x} y} & \sigma_{y y} & \sigma_{y^{\prime} y} & \sigma_{t y} & \sigma_{E y} \\
\sigma_{x y^{\prime}} & \sigma_{x^{\prime} y^{\prime}} & \sigma_{y y^{\prime}} & \sigma_{y^{\prime} y^{\prime}} & \sigma_{t y^{\prime}} & \sigma_{E y^{\prime}} \\
\sigma_{x t} & \sigma_{x^{\prime} t} & \sigma_{y t} & \sigma_{y^{\prime} t} & \sigma_{t t} & \sigma_{E t} \\
& & & & & \\
\sigma_{x E} & \sigma_{x^{\prime} E} & \sigma_{y E} & \sigma_{y^{\prime} E} & \sigma_{t E} & \sigma_{E E}
\end{array}\right),
$$

where $E$ and $t$ are the longitudinal coordinates (relative to $E_{\text {ref }}$ and $t_{\text {ref }}$ of the reference particle). The transverse normalized emittance can be calculated from the determinant of the 4D covariance matrice in Equation 2.16,

$$
\varepsilon_{\perp}=\frac{p_{\mathrm{ref}}}{m_{\mu}}\left|\Sigma_{4 \times 4}\right|^{\frac{1}{4}}
$$


Similar relation holds for the 6D normalized emittance $\left(\varepsilon_{6 D} \propto \frac{p_{\text {ref }}}{m_{\mu}}\left|\Sigma_{6 \times 6}\right|^{\frac{1}{6}}\right)$. Generally, for beam cooling studies, the aim is to measure the change in a beam quantity that changes only as a result of cooling. When a beam evolves in an accelerating structure, it changes its shape. However, normalized emittance is conserved during this process unless there is a material in the way of the beam (note that the 4D emittance is not always conserved and its conservation depends on the change in the longitudinal phase-space). Thus, two figures of merit for beam cooling in MICE are the six-dimensional (transverse and longitudinal) and four-dimensional (transverse) normalized emittance reductions. To demonstrate cooling, the normalized emittance of the initial distribution upstream of the absorber is computed and compared with the normalized transverse emittance of the final distribution after passing through the absorber (downstream). In general, a good grasp of energy loss and multiple scattering of muons in absorbing material is essential for understanding ionization cooling. In a typical ionization cooling channel, the muon beam transverse and longitudinal momenta are reduced in an absorber followed by a subsequent restoration of the beam longitudinal momentum using an RF cavity (Fig. 2.7). One of the main purposes of MICE is studying the beam transverse volume reduction. The beam cooling equation below describes the rate of change of the normalized transverse emittance [28]:

$$
\frac{d \varepsilon_{\perp}}{d x} \cong-\frac{\varepsilon_{\perp}}{\beta^{2} E_{\mu}}\left\langle\frac{d E}{d x}\right\rangle+\frac{\beta_{\perp}(13.6 \mathrm{MeV} / c)^{2}}{2 \beta^{3} E_{\mu} m_{\mu} X_{0}}
$$

where $E_{\mu}$ is the muon energy, $\beta c$ the muon velocity, $d E / d x$ the magnitude of the average ionization energy loss rate, $m_{\mu}$ the muon mass, $X_{0}$ the radiation length, and $\beta_{\perp}$ the transverse beta functions, at the absorber. The first term in the equation represents cooling from ionization energy loss and the second term describes heating from multiple Coulomb scattering. The minimum achievable emittance, or equilibrium emittance, can be optimized experimentally and for a given material and focusing conditions is obtained by setting the rate of change of the normalized transverse 

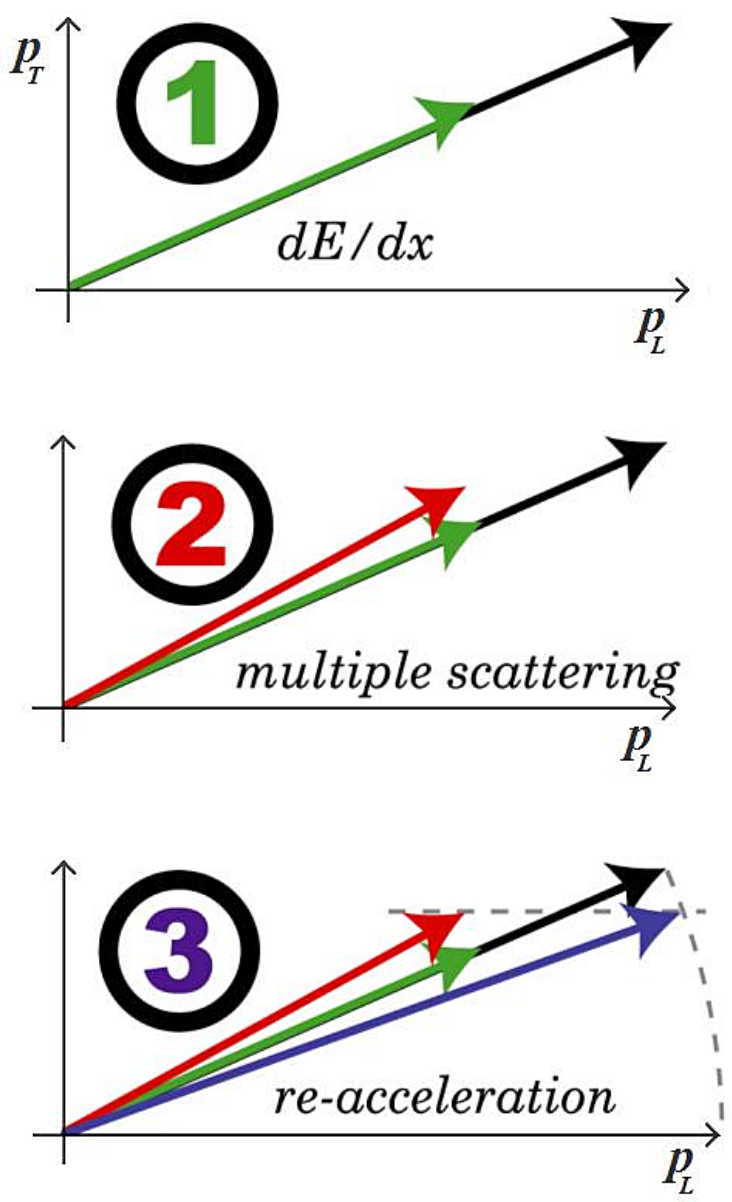

Figure 2.7. Ionization energy loss in material reduces the beam momentum in all directions (1), and the subsequent acceleration from RF cavity replaces the longitudinal momentum (2). The energy loss is concurrent with the multiple scattering (3) which in turn heats the beam by slightly changing the direction of the momentum vector [28]. 
emittance to zero:

$$
\varepsilon_{\perp} \cong \frac{\beta_{\perp}(13.6 \mathrm{MeV} / c)^{2}}{2 X_{0} \beta m_{\mu}}\left\langle\frac{d E}{d x}\right\rangle^{-1}
$$

A smaller equilibrium emittance compared with a constant input emittance (or a larger input beam emittance compared with a constant equilibrium emittance) leads to a more effective emittance reduction. As shown in Equation 2.20, a smaller equilibrium emittance is achieved when the beta function, $\beta_{\perp}$ is minimized, and $X_{0} d E / d x$ factor maximized [28]. In MICE, a small beta function is achieved by focusing the beam tightly at the absorber using solenoids. Large radiation length for a given energy loss is achieved by using low- $Z$ absorbing materials such as LiH (lithium hydride) and $\mathrm{LH}_{2}$ (liquid hydrogen).

Energy loss is a stochastic process; there are fluctuations in the amount of energy loss. Because of these fluctuations, referred to as "straggling," the transverse cooling is often accompanied by longitudinal heating. What follows in the next paragraphs cover the various longitudinal cooling schemes, including the scheme best suited for achieving longitudinal cooling at a future muon collider, the "emittance exchange".

The normalized longitudinal emittance is defined as [36],

$$
\varepsilon_{n \|}=\beta_{\|} \gamma \delta \sigma_{z}
$$

where $\sigma_{z}$ is the beam bunch length, $\gamma$ the relativistic Lorentz factor $(E / m)$, and $\delta$ the momentum spread ( $\delta=\frac{\sigma_{p z}}{p z}$ where $\sigma_{p z}$ is the RMS momentum spread). The change in the longitudinal emittance as a muon traverses a distance $d x$ in material (derivation described in [36]) is given by [36],

$$
\frac{d \varepsilon_{n \|}}{d x}=\frac{\beta_{\|} \gamma \delta \sigma_{z}}{p_{z}} \frac{d \sigma_{p z}}{d x} .
$$

Three effects can modify the rate of change of the momentum spread, $\frac{d \sigma_{p z}}{d x}$. The first is the momentum-dependent energy loss feature shown in Figure 2.3; this leads to 
the following $\frac{d \sigma_{p z}}{d x}$ term [36],

$$
\frac{d \sigma_{p z}}{d x}=\frac{\sigma_{E}}{\beta c} \frac{d}{d E}\left(\frac{d E}{d x}\right)
$$

where $\sigma_{E}$ is the RMS energy spread and $\beta c$ the muon velocity. The momentumdependence feature exists because of the curvature of the $d E / d x$ curve where muons of different momenta lose different amounts of energy; energy loss increases for muons of momenta below the ionization minimum, causing longitudinal heating, while it decreases for energies above it (in the relativistic-rise region), causing longitudinal cooling. It is, therefore, possible to rely on the muon momenta above $300 \mathrm{MeV} / \mathrm{c}$ for longitudinal cooling; however, the issue with this approach is that the rate of cooling in this region is minimal (due to the small slope of the $d E / d x$ curve).

The straggling also affects the rate of change of the beam energy spread [36] leading to the following $\frac{d \sigma_{p z}}{d x}$ term,

$$
\frac{d \sigma_{p z}}{d x}=\frac{F}{2 \beta c \sigma_{E}} \gamma^{2}\left(1-\frac{\beta^{2}}{2}\right)
$$

where $\sigma_{E}$ is the RMS energy spread, $\beta c$ the muon velocity, and $F$ a materialdependent factor $\left(F=\frac{4 \pi\left(r_{e} m_{e} c^{2}\right)^{2} N_{A} Z \rho}{A}\right.$ where $m_{e}$ is the mass of the electron, $r_{e}$ the classical radius of the electron, $Z$ the atomic number of the material, $\rho$ the material density, and $A$ the atomic weight of the material). Equation 2.24 represents the heating term, and to minimize it, longitudinal cooling for lower muon momenta are preferred (the growth in energy spread $\propto \gamma^{2}$ ) [36].

Finally, let us describe the third effect that can modify the rate of change of the momentum spread: the emittance exchange scheme used in the six-dimensional cooling channels (Fig. 2.8). When a wedge-shaped absorber (with transverse thickness variation) is placed at a location of non-zero beam dispersion, the higher-energy particles of the beam passing through the absorber lose more energy than lowerenergy particles. The rate of change of the longitudinal momentum spread for this 


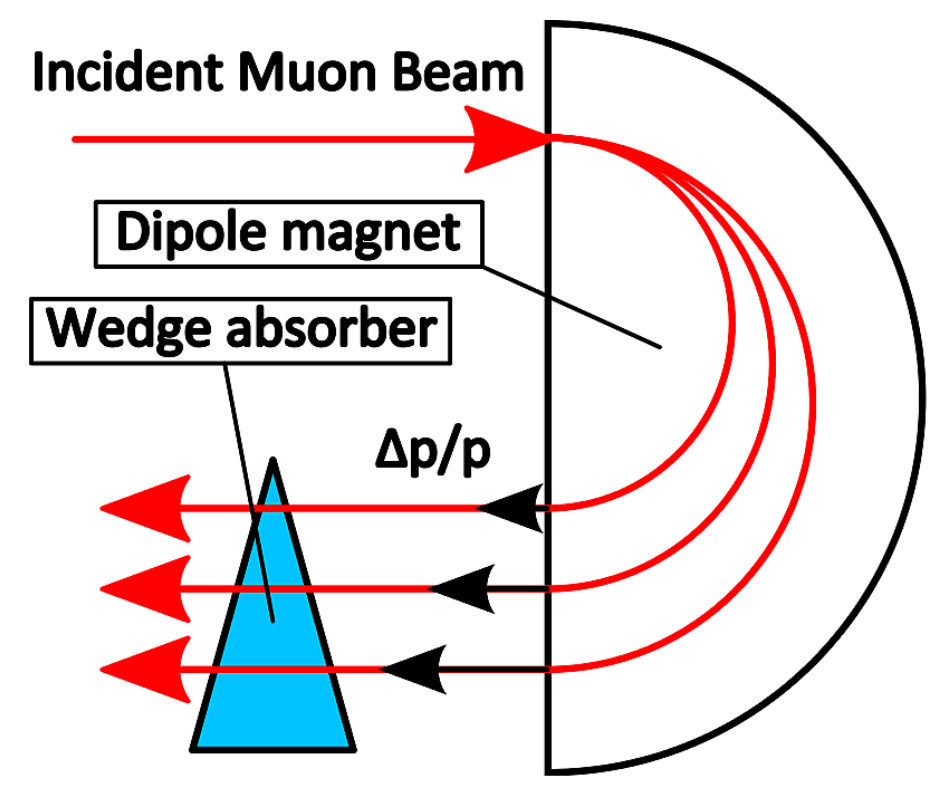

Figure 2.8. Process of emittance exchange via a wedge-shaped absorber. Courtesy of Muons, Inc. [37]

effect becomes [36],

$$
\frac{d \sigma_{p z}}{d x} \cong \frac{1}{\beta c} \frac{d E}{d x} \frac{\eta \delta}{\alpha L}
$$

where $\eta$ is the dispersion $\left(\eta=\frac{d x}{d \delta}\right), \alpha$ the wedge angle $\left(\alpha=\frac{d x}{d x}\right)$, and $L$ the wedge thickness for a wedge at position $x=0$. This effect, known as direct emittance exchange causes the transverse and longitudinal phase spaces to get exchanged. One possible direct emittance exchange scheme is shown in Figure 2.8. A dipole magnet introduces dispersion (energy-position correlation) in the beam to allow higher momentum muons to pass through the thicker part of the wedge. By adding dispersion, the beam width is increased while keeping energy spread constant [36]. The MICE muon beam has very small natural dispersion. This dispersion is not sufficient for demonstrating emittance exchange (emittance exchange using MICE simulated lattice is covered in Section 4.5). In general, the ionization cooling process with a combination of wedge and flat absorbers and RF cavities can lead to a very small longitudinal emittance. At that point, the bunch becomes too short for matching the beta function at the interaction point [28], which leads to the beam luminosity reduction. Reverse emittance exchange can reduce the transverse emittance to achieve 


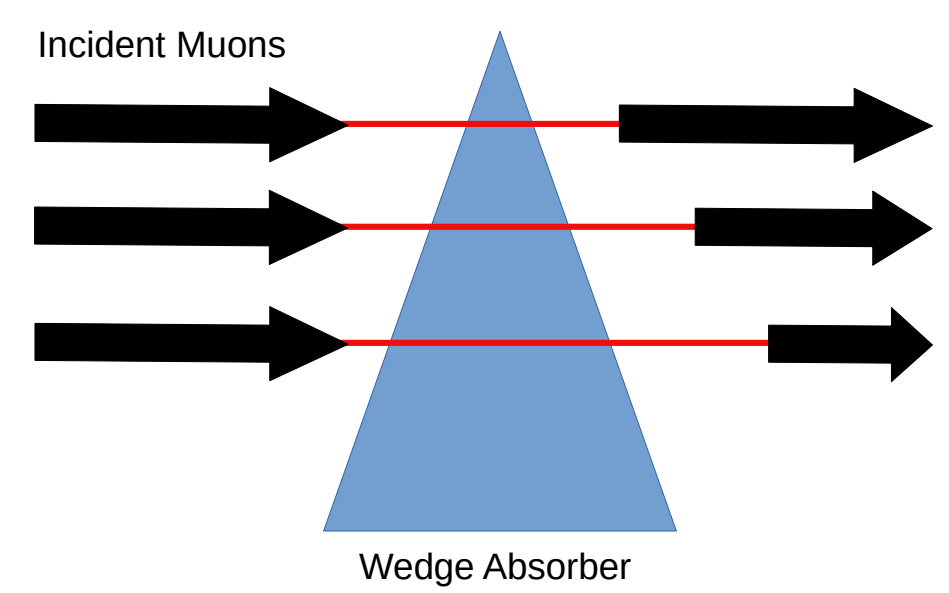

Figure 2.9. Process of reverse emittance exchange via a wedge-shaped absorber. Courtesy of Muons, Inc. [37].

high luminosity at the expense of heating the longitudinal emittance and lengthening the bunch. As shown in Figure 2.9, a beam with large transverse emittance but small momentum spread is passed through the wedge; the wedge introduces a momentumposition correlation in the beam, causing the transverse emittance to reduce while increasing the longitudinal emittance. First experimental demonstration of reverse emittance exchange using MICE data is covered in Section 5.2.

\subsection{The Experiment}

The MICE experiment consists of several components: a beamline (Fig. 2.10), a series of particle identification detectors, tracking detectors, and a cooling section (Fig. 2.11). These components are described below.

2.2.1 Beamline. The proton beam for muon production in MICE is supplied by the ISIS proton synchrotron situated at Rutherford Appleton Laboratory. In ISIS, protons with an initial kinetic energy of $70 \mathrm{MeV}$ are accelerated to $800 \mathrm{MeV}$. MICE operates parasitically on the ISIS proton beam; the MICE titanium target is dipped into the low-density periphery of the proton beam [38]. While the MICE target is in the ISIS proton beam, the protons hit the target nucleons and produce pions, along 


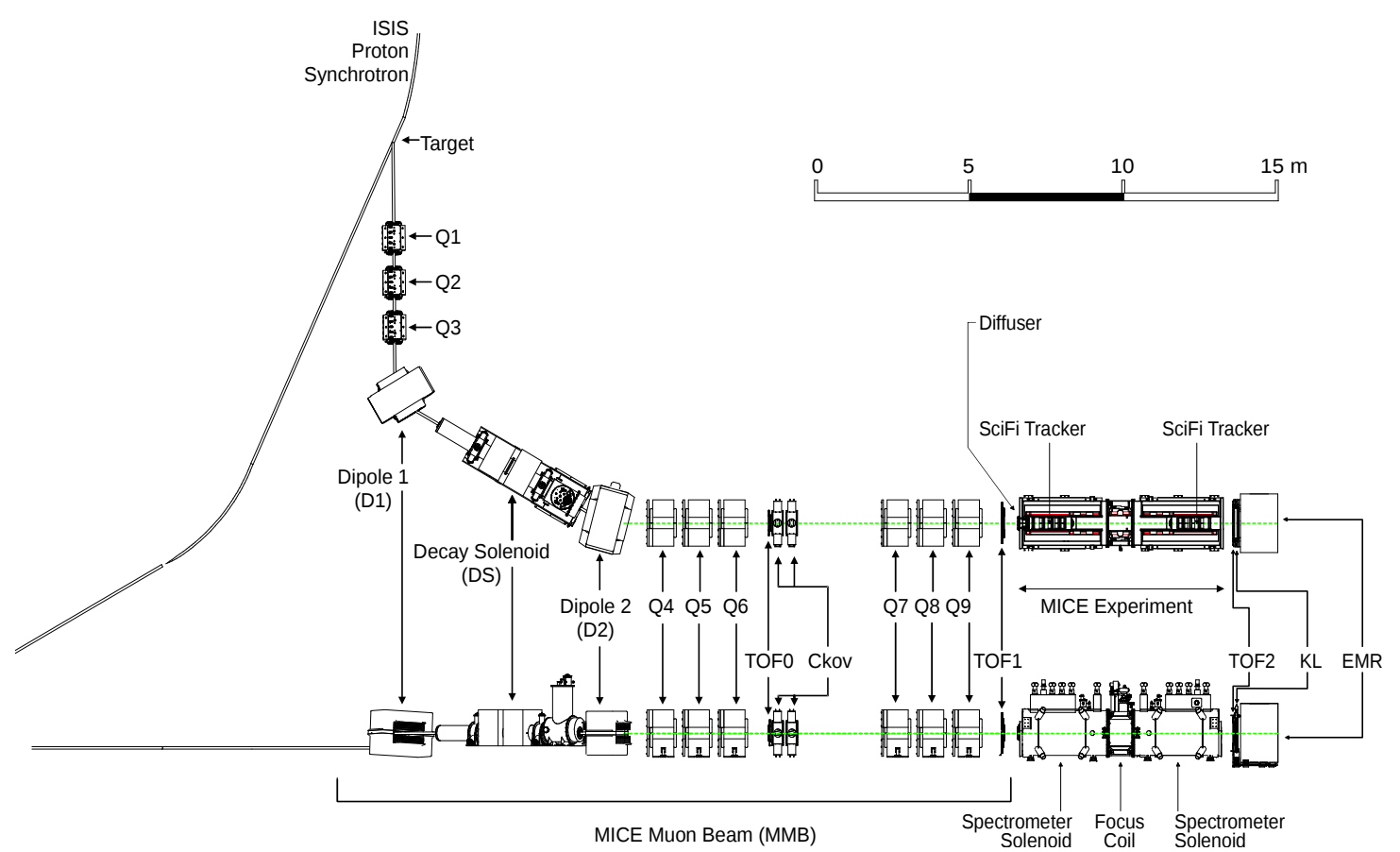

(a)

(b)

Figure 2.10. Schematics of the MICE muon beamline.

with forward-directed protons and neutrons. Pions as well as protons and neutrons pass through a vacuum window into the MICE beamline (Fig. 2.10). To avoid losing particles (muons or pions) in the MICE beamline, two triplets of quadrupole magnets (Q1, Q2, Q3 and Q4, Q5, Q6) are used to focus the beam [39]. In addition to quadrupole magnets, two bending magnets (D1 and D2) are used for momentum selection [39]. Through an interplay of the two dipoles, the desired momentum spectrum and particle composition in the MICE beam can be achieved (Fig. 2.12). Along
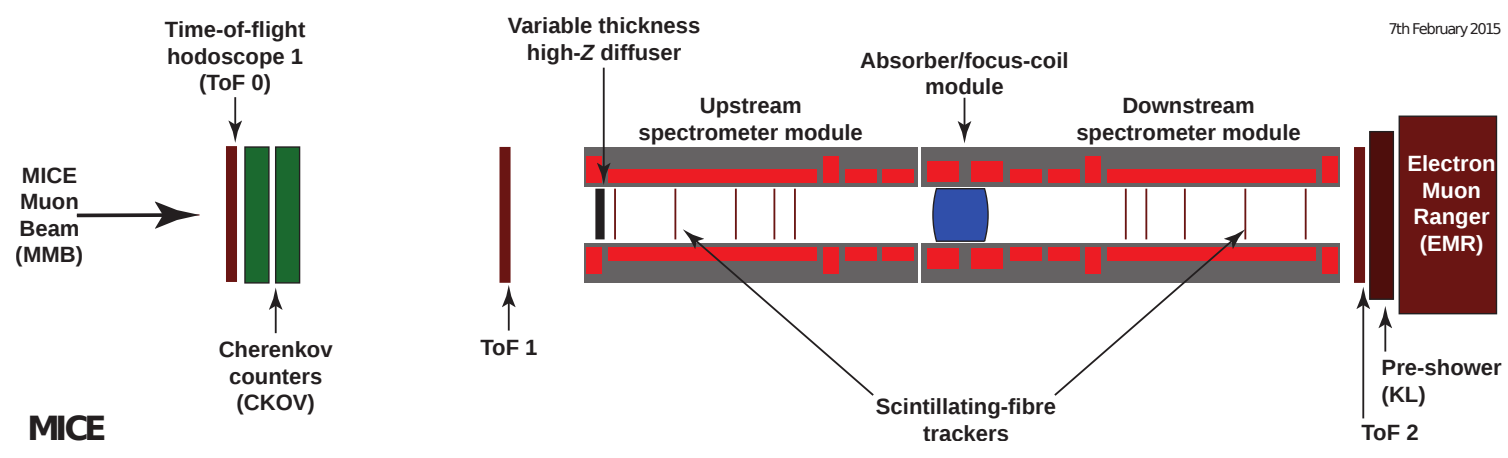

Figure 2.11. Schematic diagram of MICE in its final configuration with upstream and downstream spectrometers surrounding a cooling cell, and particle identification detectors (ToF, Cherenkov, KL, and EMR). 


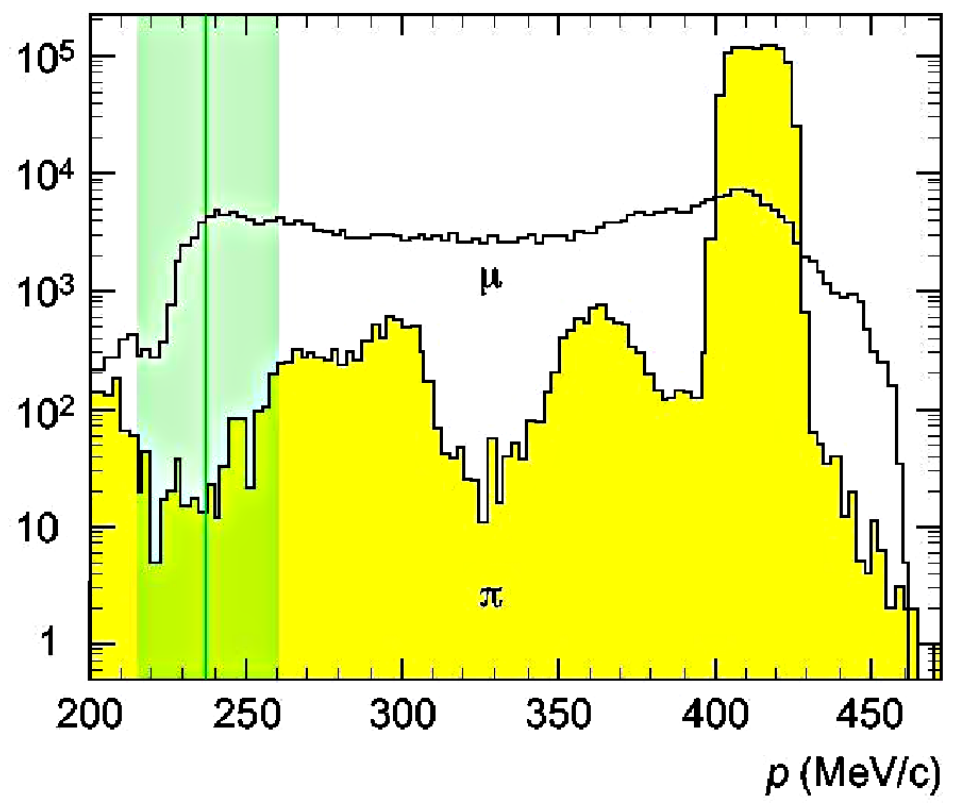

Figure 2.12. MICE beam downstream of decay solenoid. The green rectangle represents the "muonic" beam setting on the figure; this beam almost purely consists of muons. The "pionic" beam setting, on the other hand, has approximately equal numbers of pions and muons with momenta in the range of 400 to $430 \mathrm{MeV} / c$ [39].

the way, the pions pass through the Decay Solenoid, DS. The DS operates at $5 \mathrm{~T}$, and its primary purpose is to capture the decaying pions and the pion-decay muons. The pion decay in flight results in a muon beam with large momentum spread at the exit of the DS, as shown in Figure 2.12. The muon momentum can vary based on the pion momentum selected using D1. The maximum and minimum muon momenta are the results of the forward and backward-decay muons. In one case, known as the "pionic" beam setting, the dipoles are set to select particles that have the same momenta in D1 as D2 $\left(B_{D 1}=2 \times B_{D 2}\right)$; this setting results in a beam that is composed of a mixture of pions, muons, and electrons. In another beam setting, D1 is set to select particles that have momenta twice as much as the particles' momenta in D2 $\left(B_{D 1} \gg 2 \times B_{D 2}\right)$. With this setting, known as the "muonic" beam setting, the backward-going muons in the pion rest frame are selected, and the resulting beam has mostly muons with a small pion contamination. A small fraction of the pions survives long enough to 


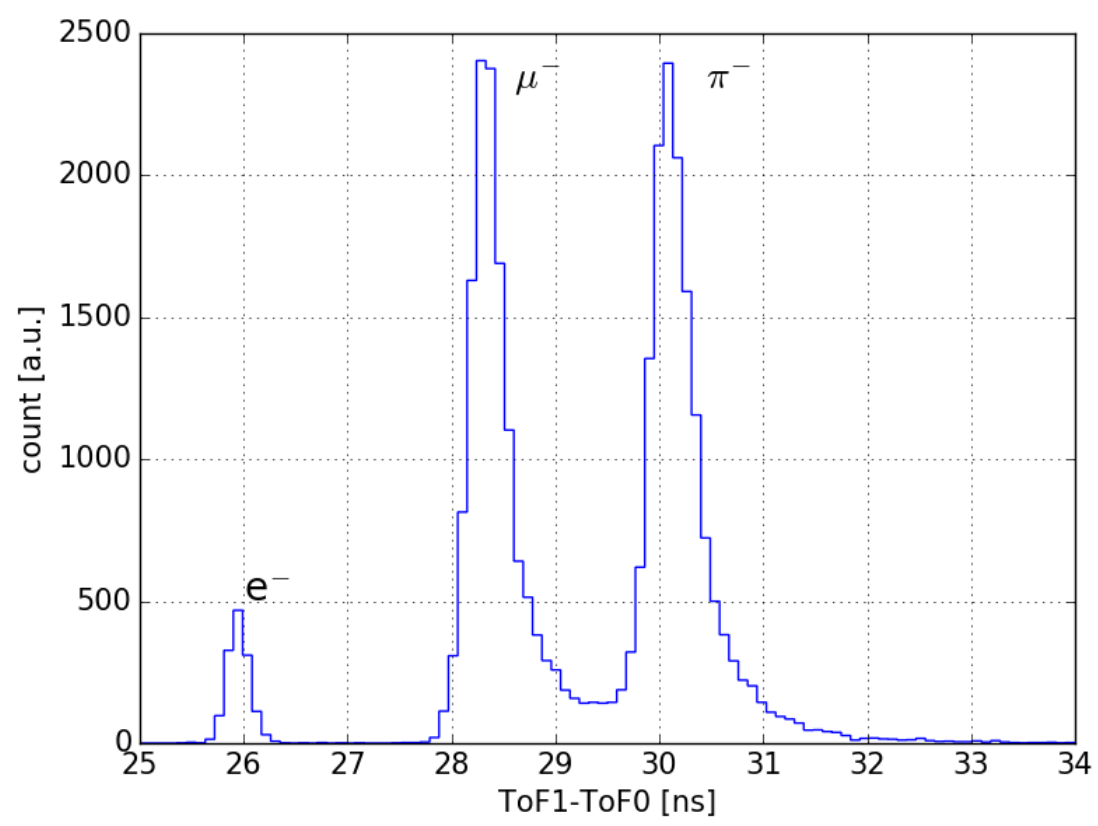

Figure 2.13. Three distinct time-of-flight peaks for electrons, muons, and pions using the time-of-flight of the particles between ToF0 and ToF1. A "pionic" beam was used to produce this plot.

get through the experiment. To ensure muon beam purity, these contaminating pions are tagged and rejected from the cooling measurement using the MICE particle identification (PID) detectors.

2.2.2 PID Detectors. Three Time-of-Flight, ToF detectors (ToF0, ToF1, and ToF2) made of scintillating slabs, discriminate between particles of the same momentum and differing masses. Between ToF0 and ToF1, there is additional focusing using another quadrupole triplet (Q7, Q8, Q9). Electrons and pions have respectively shorter, and longer times of flight than muons between pairs of ToF detectors, and, as a result, there are three distinct time-of-flight peaks for electrons, muons, and pions for a "pionic" (Fig. 2.13). In the case of a "muonic" beam (Fig. 2.14), the ToF peak corresponding to the pions is mixed in with the muon peak. There are also two Cherenkov counters in MICE with aerogel of differing refractive indices. The different 


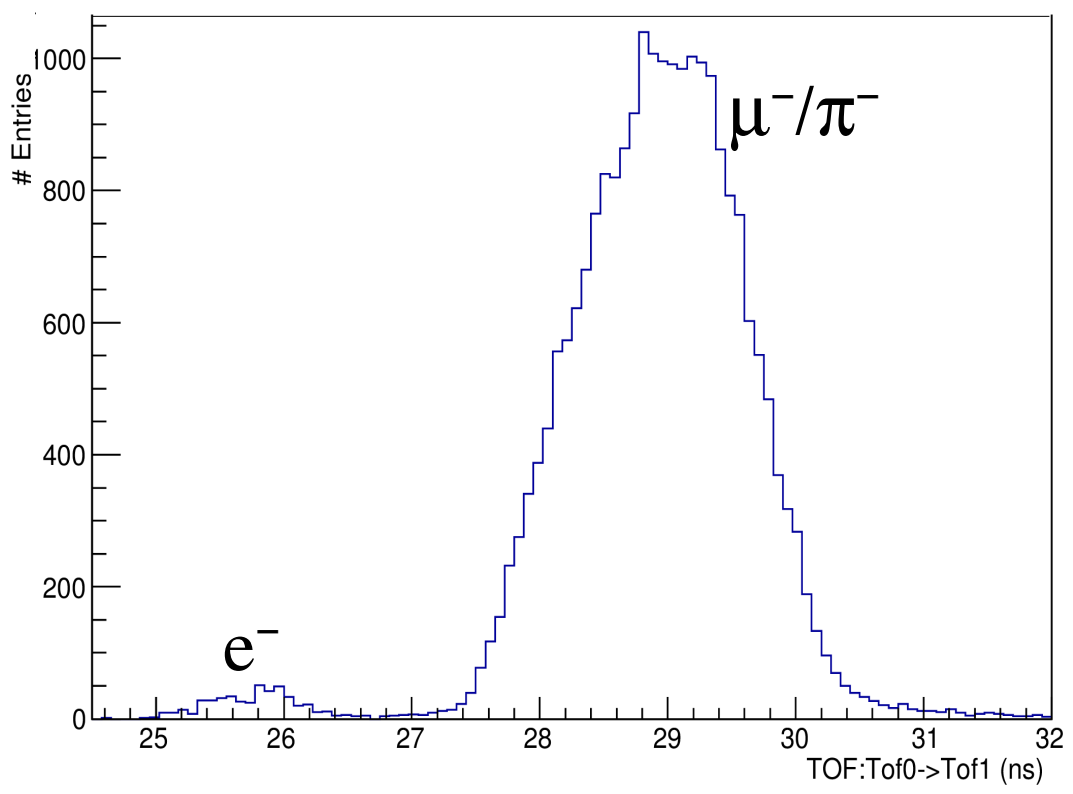

Figure 2.14. Two distinct time-of-flight peaks for electrons and muons/pions using the time of flight of the particles between ToF0 and ToF1. A muonic beam was used to produce this plot. To better distinguish between pions and muons, other PID detectors must be used.

indices of refraction impose different momentum thresholds on the particles.

The KLOE-light detector, KL is a calorimeter and uses lead foil interspersed with scintillating fibers to differentiate between particles based on their ADC counts (the analog-to-digital converter signal received by the MICE data acquisition system when the particles interact with KL scintillating slabs). As shown in Figure 2.15, pions have a longer-tailed ADC distribution compared to muons; this is because they interact hadronically with the lead in the KL [40] (as opposed to an electromagnetic interaction). The most downstream PID detector is the Electron Muon Ranger, EMR, which is calorimeter composed of planes of scintillating bars. Different particles have different track topologies in the EMR. Electrons shower in the EMR, leaving some planes unhit ( $\rho<1$ in Fig. 2.16), in contrast to muon tracks, which consistently hit all planes along their path $(\rho=1$ in Fig. 2.16) through the EMR [41]. The 


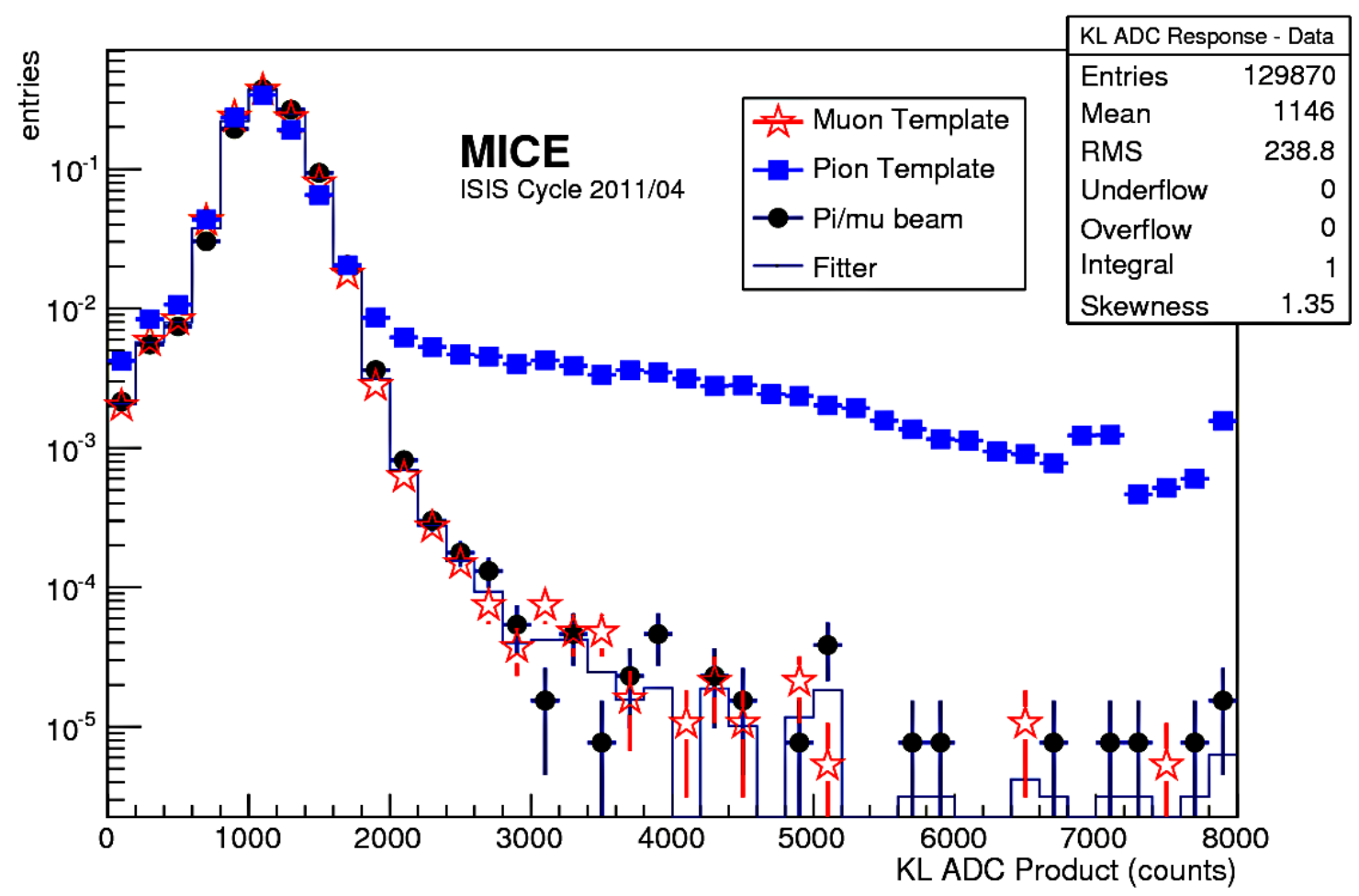

Figure 2.15. The ADC responses of muons and pions as they interact with the KL detector. The ADC distribution indicates that pions have larger tails compared to muons and that the majority of the beam particles in the MICE beam are muons, closely following the muon template. The pi/mu beam is a pionic beam [40].

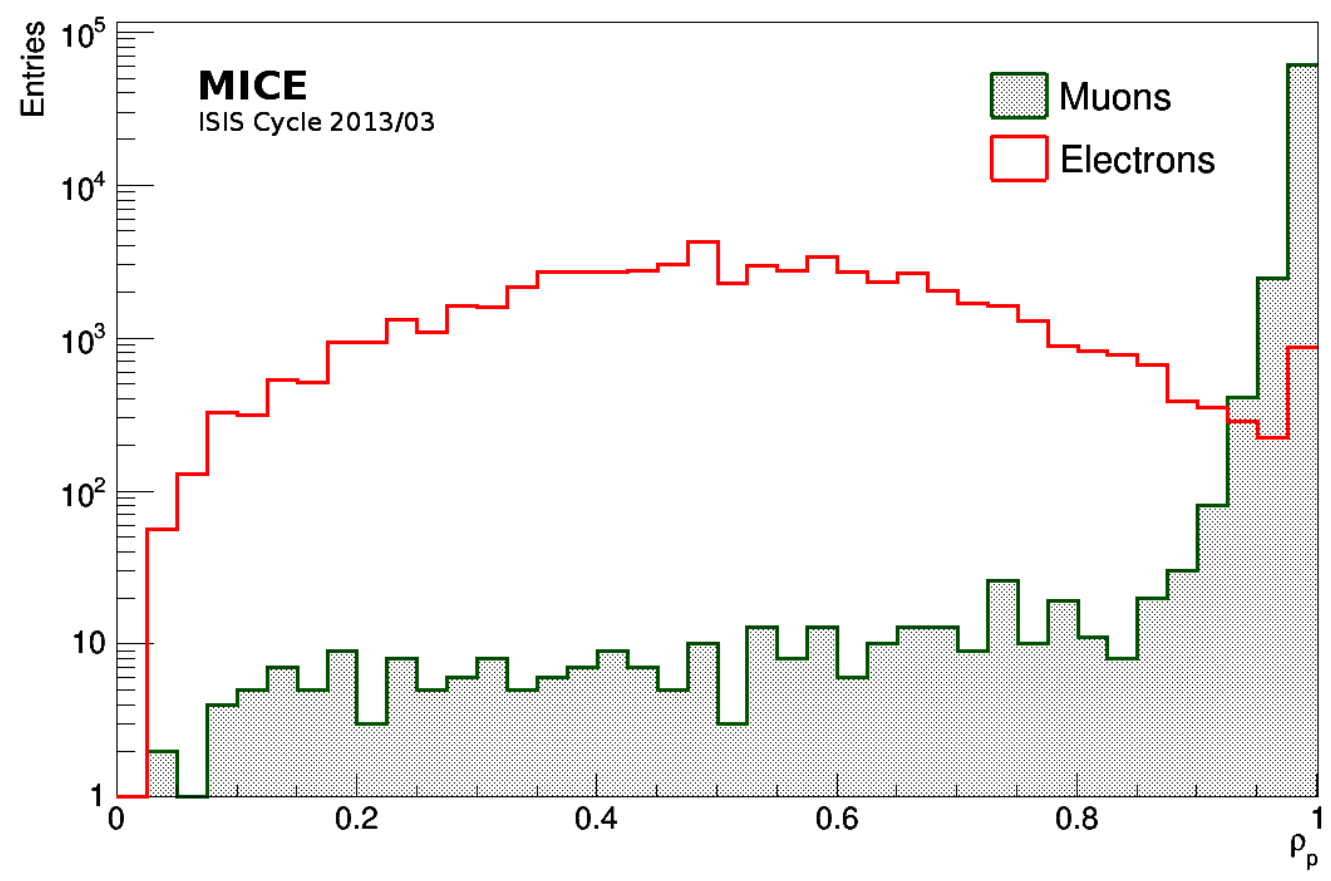

Figure 2.16. Muon and electron plane density distributions in the EMR [41]. 
EMR parameter used for this purpose is the plane density, $\rho$, which represents the average number of planes hit as a particle traverses the detector. The electron tag efficiency using the EMR is about $98.6 \%$, and the pion contamination measured using a combination of $\mathrm{ToF}$ and $\mathrm{KL}$ detector is less than 1.4\%; this ensures a cooling measurement on a pure muon beam.

2.2.3 Trackers and the Cooling Channel Magnets. MICE has two identical scintillating fiber tracking detectors, one upstream and one downstream of the absorber [42]. The trackers are used for position and momentum measurement, from which beam emittance, phase-space density, phase-space volume, and single-particle amplitude can be reconstructed. Each tracker (see Fig. 2.17) is composed of five planar scintillating-fiber stations. For measurement of beam cooling, the input and output beam distributions at the tracker stations immediately upstream and downstream of the absorber (tracker reference planes), are compared. Each tracker station has three doublet fiber layers, referred to as the "u," "v," and "w" planes, oriented at 120 degrees with respect to each other. Each tracker plane has 214 fibers. The orientation of the doublet layers and the fibers is shown in Figure 2.18; the fibers are grouped in gangs of seven for a single readout channel, and the scintillation signals arising from a particle interaction are read out by visible light photon counters (VLPCs) [42]. ${ }^{24}$ To ensure a small multiple Coulomb scattering in the trackers, the scintillating fiber diameter is only $350 \mu \mathrm{m}$. The trackers have a spatial resolution of about $470 \mu \mathrm{m}$ and a transverse momentum resolution of $1 \mathrm{MeV} / c$ [42].

Each tracker is immersed in the solenoidal field of a Spectrometer Solenoid (SS) (a photo of one SS is shown in Fig. 2.19). The upstream and downstream SS have five superconducting coils with two used for matching the muon beam into

${ }^{24} \mathrm{~A}$ VLPC is a silicon diode with high quantum efficiency and capable of converting single photons to many photoelectrons. 


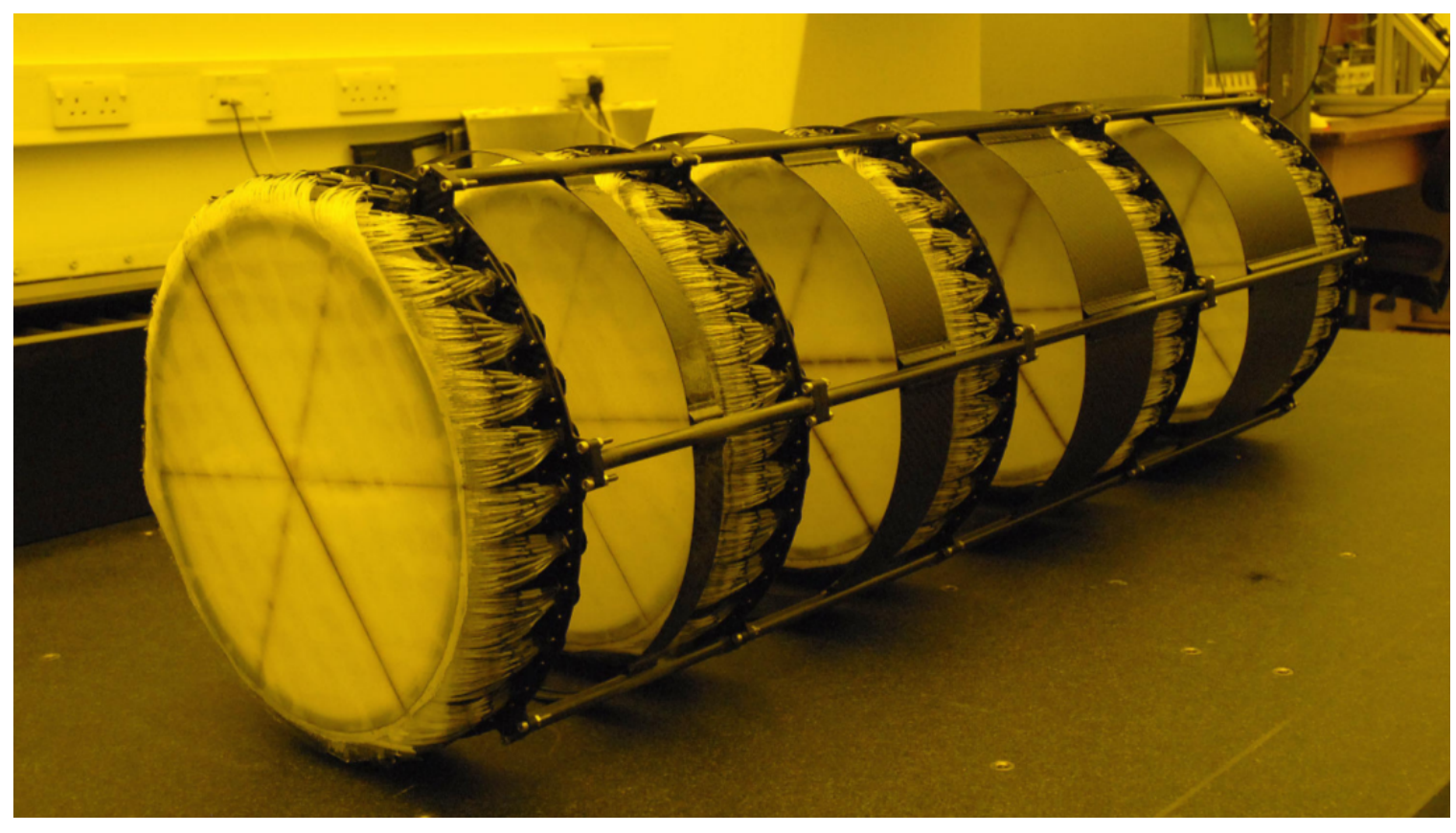

Figure 2.17. MICE scintillating fiber tracker.
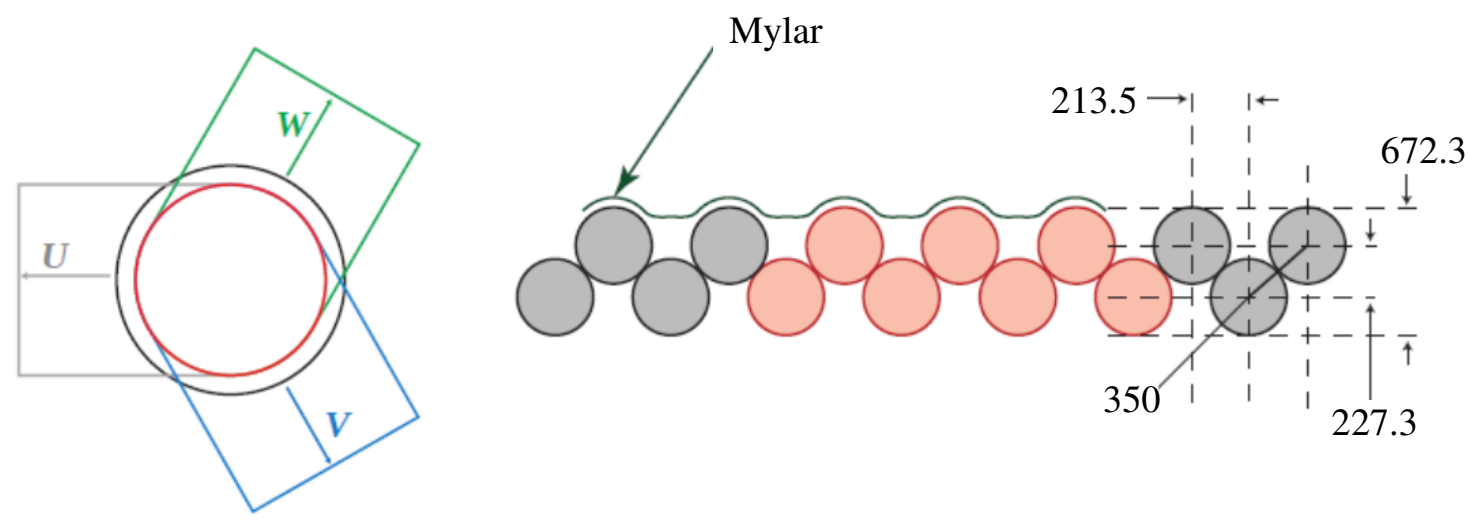

Figure 2.18. The layout and orientation of the doublet fiber layers in the MICE trackers [42]. 


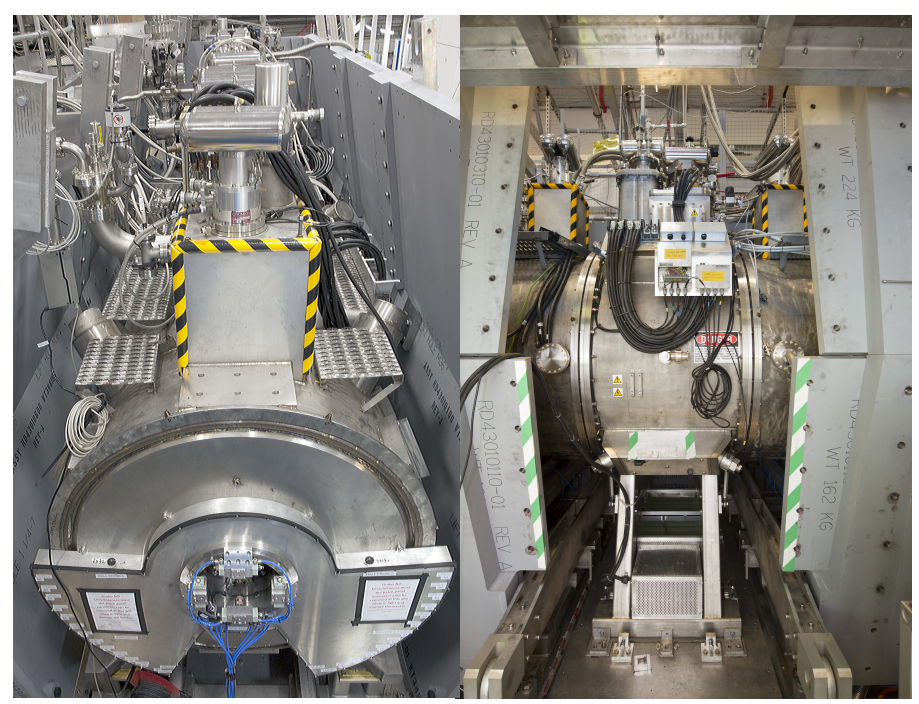

Figure 2.19. Photos of the SS module sitting inside the partial return yoke (left) and the AFC module (right). The AFC module is typically covered by the partial return yoke but at the time of the photo, it was removed (absorber changed in progress).

and out of the absorber (Match 1 and Match 2 coils), and three for maintaining uniform fields in the tracking volumes (End 1, Center, and End 2 coils). Because of the fields in the tracking volumes, any particle traversing the trackers form helical tracks (Fig. 2.20). To reconstruct the momentum coordinates of each particle, the reconstruction algorithm in the MICE Analysis User Software (MAUS) [43] performs a series of cluster-finding, space-point reconstruction, and track-finding operations on MICE simulated or experimental data. The cluster-finding algorithm looks for one or two neighboring readout channel hits to form clusters; the choice of no more than two neighboring channels for cluster finding is due to the readout arrangement, in which one particle can register hits in two neighboring channels. The clusters are then used to form spacepoints. A track-finding algorithm then performs a search over the spacepoints in the five stations and fits the spacepoints into a helical or a straight track using a linear-least-squares fitting. The transverse and longitudinal momentum resolutions are 1 and $4 \mathrm{MeV} / c$. For helical track reconstruction, in the transverse direction, the reconstruction algorithm fits a circle to each particle trajectory to reconstruct the transverse momentum coordinates $\left(p_{x}, p_{y}\right)$ (Fig. 2.20); this is done 


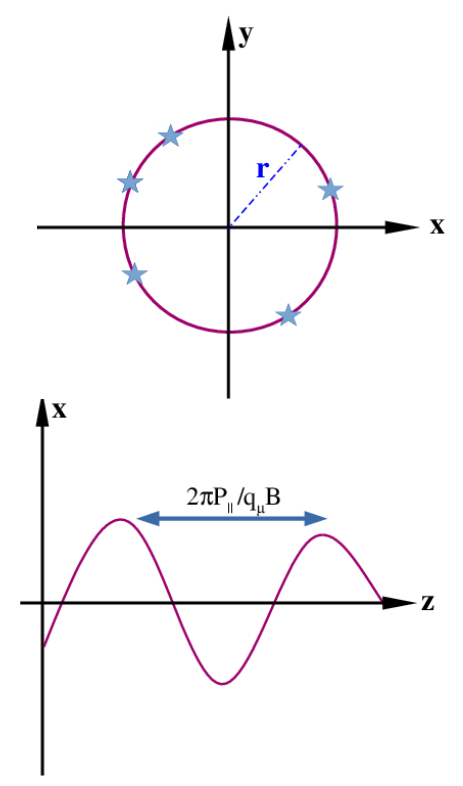

Figure 2.20. Momentum reconstruction in the transverse plane (upper diagram) and the longitudinal direction (lower diagram). $r$ represents the radius of the helix $\left(r=\frac{p_{\perp}}{q B}\right)$. The same track would have a sinosoidal shape in the longitudinal direction.

using the knowledge of the transverse position coordinates, $x$ and $y$, of the particles hitting the fibers and the solenoid field, $B$ (ignoring the initial phase):

$$
\begin{aligned}
& x(t)=-\frac{p_{\perp}}{q B} \cos \left(\frac{q B t}{m}\right), \\
& y(t)=-\frac{p_{\perp}}{q B} \sin \left(\frac{q B t}{m}\right),
\end{aligned}
$$

where $t$ is the time, $m$ the mass of the particle, $q$ is the charge. In the longitudinal direction, one option is perform a nonlinear sinusoidal fit. A more straightforward approach is to use the results of the circle fit; first, the rotation for each spacepoint is estimated, and then a fit to the path length of the track is performed [44],

$$
z(t)=\frac{p_{\|} t}{m_{\mu}} .
$$

In addition to the SS module, MICE has an absorber focus coil module (AFC) shown in Figure 2.19; placed between the two trackers and the SS modules, it holds the absorber and provides the necessary focusing (or $\beta$ function) for optimum beam cooling. The SS modules can produce a magnetic field up to $4 \mathrm{~T}$, while the AFC modules are typically set at slightly lower fields (around $2 \mathrm{~T}$ ). 


\section{CHAPTER 3}

\section{NON-PARAMETRIC DENSITY ESTIMATION: A NOVEL APPROACH TO MEASURING MUON BEAM COOLING}

\subsection{Motivation}

The aim of MICE is to characterize the muon ionization cooling with high precision. Measuring the reduction in normalized RMS emittance is one way of doing this (nominally $5 \%$ emittance reduction with a precision of $0.1 \%$ ) [20]. RMS emittance measurement, however, relies on the assumption that the underlying beam distribution is Gaussian. Such an assumption in the presence of chromatic effects in the beam optics and non-Gaussian beam distribution leads to an inaccurate measurement of beam cooling. One solution is to use non-parametric density estimators (DE) where fewer assumptions are made about the underlying beam distribution. This chapter extensively covers the topic of non-parametric density estimation; Section 3.2 covers various examples of univariate and multivariate non-parametric density estimation techniques and Section 3.3 elaborates on the validation of these techniques.

\subsection{Conceptual Foundation}

Phase-space density is an important concept in describing the state of an ensemble of particles (e.g., a charged particle beam). The phase-space density can be described as the probability density function (PDF) of phase-space coordinate(s). From the general definition of a PDF, the density, $f(x)$, of a random variable $X$ is defined as

$$
f(x)=\lim _{h \rightarrow 0} \frac{1}{2 h} P(x-h<X<x+h),
$$


where $P(x-h<X<x+h)$ is the probability that $X$ lies in an interval of width $2 h$. Therefore, a natural way of estimating the PDF given a finite dataset is to estimate $P(x-h<X<x+h)$ as the fraction of data points (out of the entire sample of size $n$ ) that end up inside an interval of width $2 h$. This leads to the following estimated density definition [45]:

$$
\hat{f}(x)=\frac{j}{2 n h},
$$

where $j$ is the number of data points inside interval of width $2 h$.

In general, there are two ways of estimating the PDF (density). One is a parametric approach in which a functional form is assumed for the underlying distribution (e.g., Gaussian or Landau) and the distribution parameters are estimated. The other is a non-parametric method where fewer assumptions about the underlying PDF are made, and the individual data points are allowed to "speak for themselves" [45]; each point contributes to forming the final density estimated. The non-parametric density estimation (DE) method is advantageous compared to the parametric method when it comes to distributions that lack a parametric model. Non-parametric DE techniques typically rely on a weight function to estimate the density (e.g., kernels, series, splines), and the level of smoothing is generally tuned using a smoothing parameter (e.g., kernel width or bin width).

One widely used DE technique is the histogram, where data points are placed inside bins of equal widths, $h$. Suppose the first bin is placed at some point $x_{0}$ on the coordinate axis; $x_{0}$ can be positive or negative (and can be the smallest value in the dataset). Then, bins of width $h$ are constructed, and to estimate the density, the ratio of the number of data points inside the bin interval $\left(x_{0}+i h, x_{0}+(i+1) h\right)$ to the bin width, $h$ is taken [45]:

$$
\hat{f}(x)=\frac{j_{i}}{n h} \quad \text { for } \quad x_{0}+i h<x<x_{0}+(i+1) h .
$$

In Equation 3.3, $h$ is the bin width, $j_{i}$ the number of data points that fall within $i^{t h}$ 
bin, $n$ the sample size, and $x$ some coordinate value residing in $i^{\text {th }}$ bin $\left(x_{0}+i h<\right.$ $\left.x<x_{0}+(i+1) h\right)$. Any changes in the bin width, $h$, and the choice of $x_{0}$ can affect the estimated density. These variations can cause the same dataset to be interpreted differently (e.g., Figs. 3.1a and 3.1b). The effect of bin placement (choice of $x_{0}$ ) cannot be eliminated by choosing a very narrow bin. Most of the bins would end up with either no data point or very few data points in them; this is particularly problematic for a multidimensional space (e.g., 4D and 6D MICE muon beam). More bins are likely to be left empty, and the estimated density depends not only on the bin size and location but also on bin orientation. It is possible to remove the dependence on the parameter $x_{0}$ from the histogram definition in Equation 3.3 [45],

$$
\hat{f}(x)=\frac{j_{i}}{n h} \quad \text { for } \quad x-h<X_{i}<x+h
$$

where $X_{i}$ is the $i^{\text {th }}$ data point; it contributes to the estimated density if it falls within the interval $2 h$. In other words, the $i^{\text {th }}$ data point contributes to $x$ only when it lies in the left half (with $x-h$ coordinate) or the right half (with $x+h$ coordinate) of the dataset. This new histogram definition ensures that the bins are centered at point $x$. It is possible to re-write $j_{i}$ in Equation 3.4 as a step function $w(x)$ (or a weight function) [45],

$$
w\left(\frac{x-X_{i}}{h}\right)=\left\{\begin{array}{c}
\frac{1}{2} \text { if }\left|x-X_{i}\right|<h \\
0 \text { otherwise }
\end{array}\right.
$$

In other words, a data point $X_{i}$ contributes a weight of $1 / 2$ to the estimated density at an arbitrary point $x$ only if its distance to $x$ is less than $h$ to the left or right of $x$. Replacing $j_{i}$ in Equation 3.4 with this weight function gives (with factor of $\frac{1}{2}$ now included in the weight function definition) the following estimated density equation $[45]$ :

$$
\hat{f}(x)=\frac{1}{n} \sum_{i=1}^{n} \frac{1}{h} w\left(\frac{x-X_{i}}{h}\right),
$$

where $X_{i}$ represents the coordinate $x$ of each individual data point $i$ in the set. 


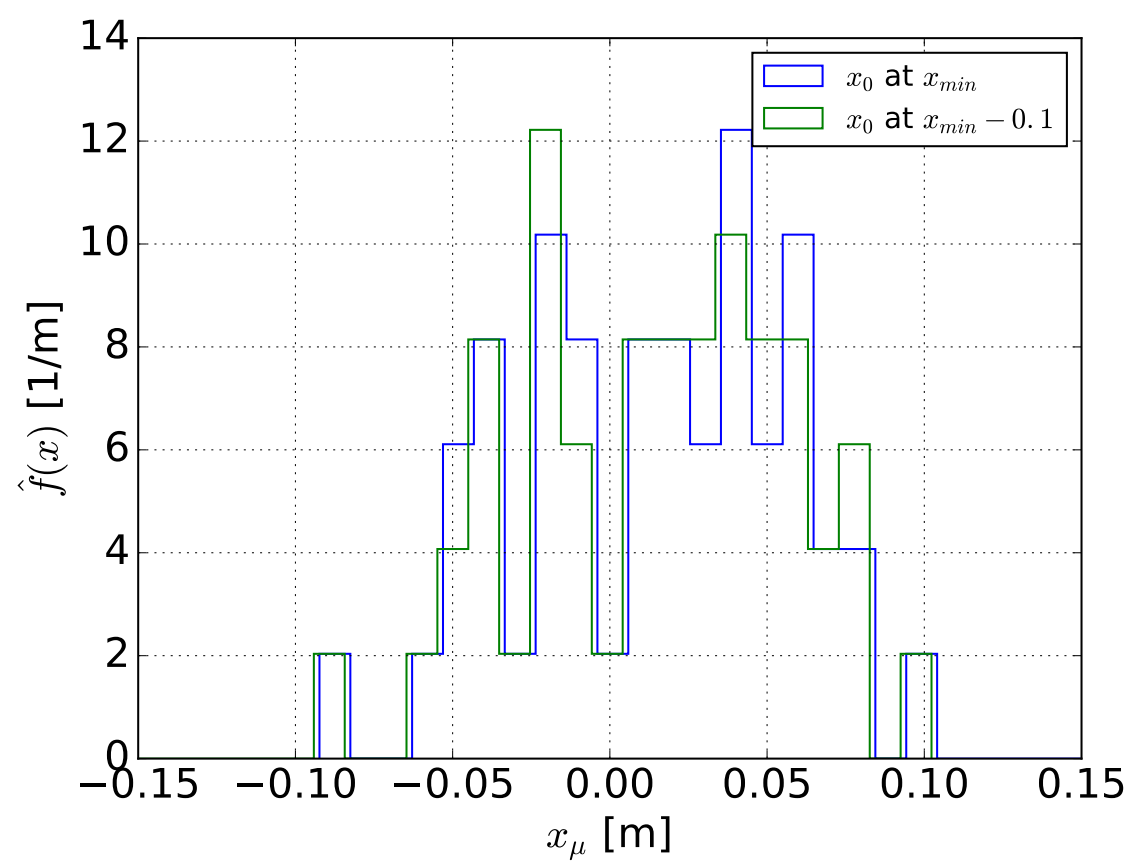

(a)

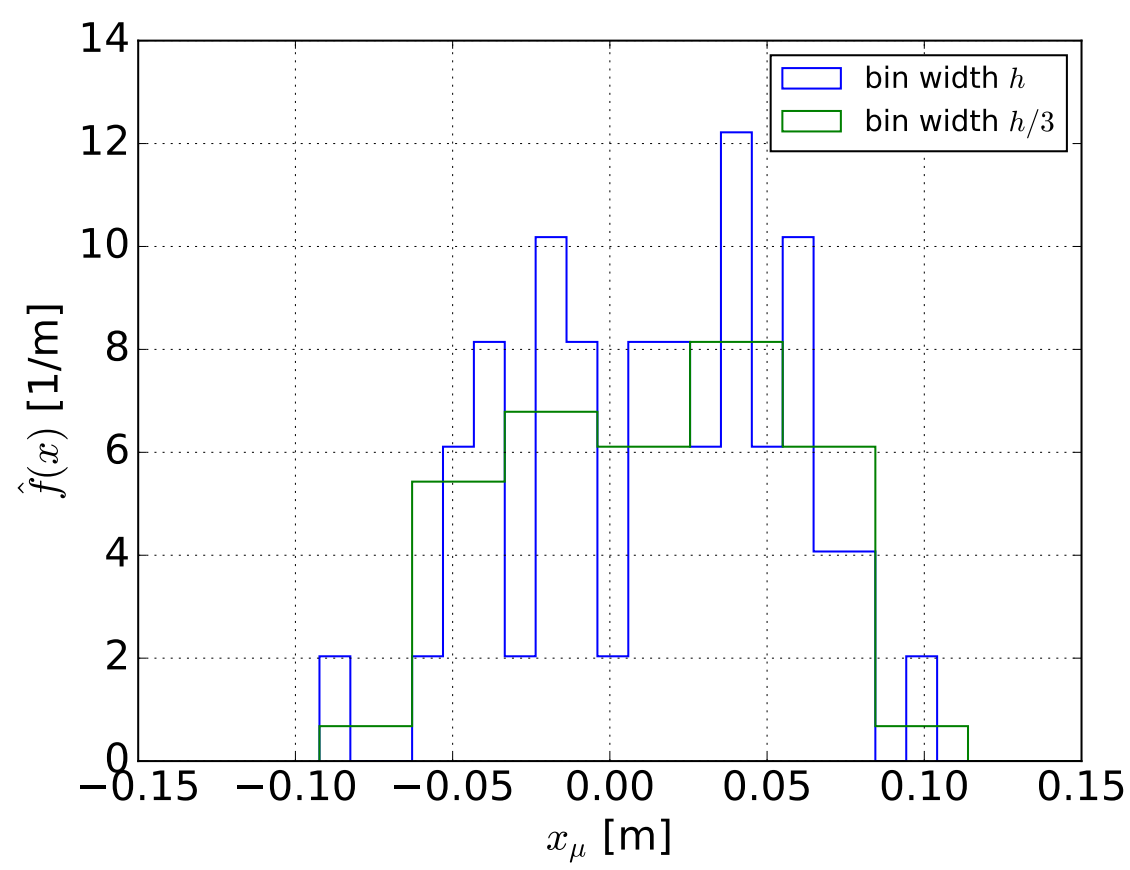

(b)

Figure 3.1. Histogram of the simulated muon $x$ positions at the entrance to the MICE cooling channel with different choices of $x_{0}$ (a) and bin width $h(h$ is) (b). For illustration purposes, the sample size is 50 . 
The weight function density estimator described in Equation 3.5 has a disadvantage from the point of view of data representation: the boundaries of the boxshaped weight function can have hard cut-offs (similar to a histogram) and every $X_{i}$ data point is either in or out of the interval $h$. The solution is to choose a smooth weight function such as a kernel function (e.g., Gaussian kernel function) with an infinite tail (that includes contributions from all data points). This way the estimated density curve is smooth and each data point in the set can contribute to point $x$ (some points contributing more than others depending on their coordinate distances to $x$ ). In one dimension, the kernel density estimator applied to some arbitrary point $x$ takes the following form [45]:

$$
\hat{f}(x)=\frac{1}{n} \sum_{i=1}^{n} \frac{1}{h} k\left(\frac{x-X_{i}}{h}\right),
$$

where $h$ is the width of the kernel function, commonly referred to as the bandwidth parameter (more on how to determine the bandwidth parameter below). In this thesis, Gaussian kernel functions are used, and in one dimension, they have the following form [45]:

$$
k\left(\frac{x-X_{i}}{h}\right)=\frac{1}{\sqrt{2 \pi}} \exp \left[-\frac{1}{2}\left(\frac{x-X_{i}}{h}\right)^{2}\right] .
$$

Substituting the Gaussian kernel function into Equation 3.7 gives [45]:

$$
\hat{f}(x)=\frac{1}{n} \sum_{i=1}^{n} \frac{1}{h} \frac{1}{\sqrt{2 \pi}} \exp \left[-\frac{1}{2}\left(\frac{x-X_{i}}{h}\right)^{2}\right],
$$

Equation 3.9 is the one-dimensional estimated density using the Gaussian kernel function (used throughout this thesis, unless stated otherwise), and Figure 3.2 illustrates how this equation works: Gaussian kernel functions of widths $h$ (each kernel width is chosen to be 0.4 ) are centered at each data point $X_{i}$. The kernel functions from all data points (all contributing to the point $x$ ) are then summed to determine the density at any point $x$.

To reiterate, the choice of the Gaussian kernel function (with an infinite tail) ensures that all data points contribute to the estimated density at a point $x$ : data 


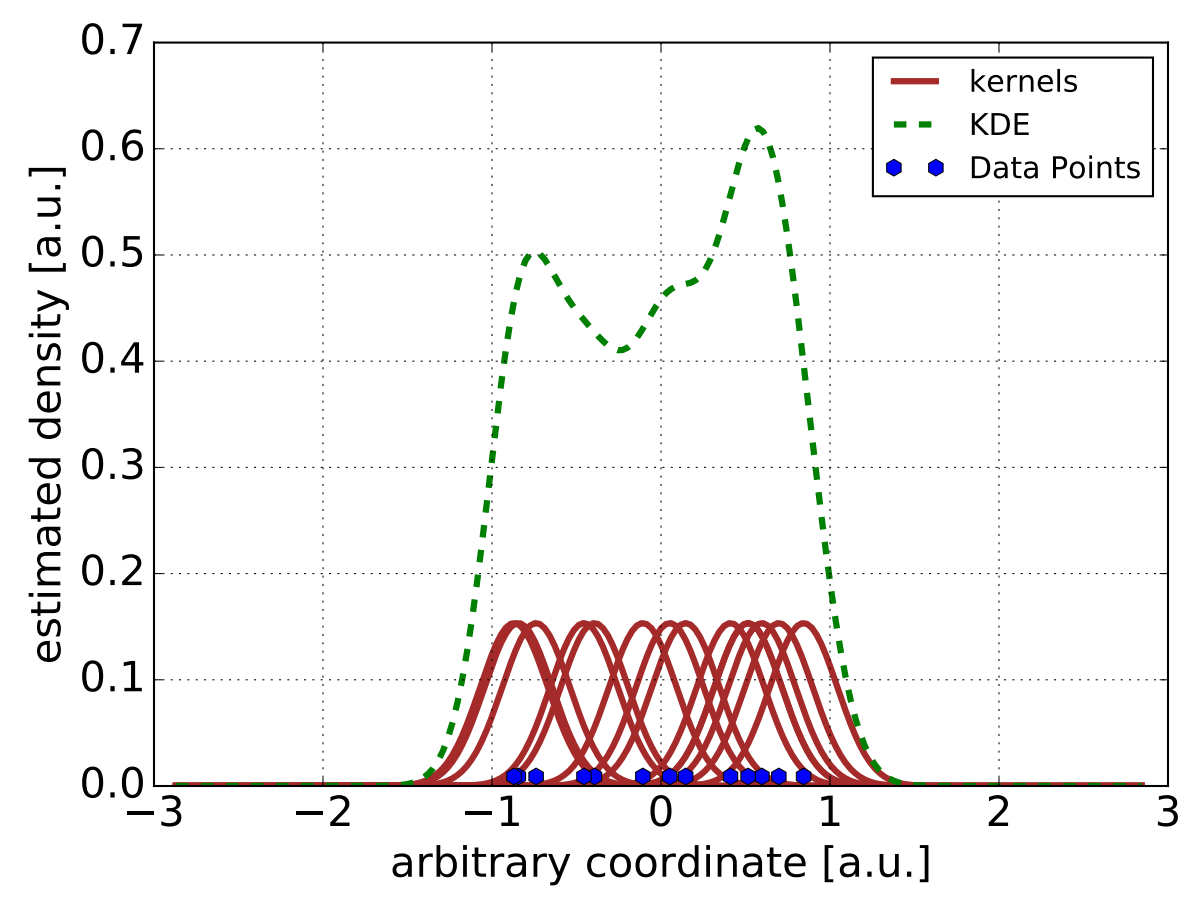

Figure 3.2. An illustration of the KDE technique in one dimension; the bandwidth parameter is 0.4 and Gaussian kernel functions are used.

points closer to the arbitrary point $x$ contribute more to $x$ than data points farther from $x . x$, commonly referred to as the reference point, can be either a data point or a grid point. Except for the contour plots (which use grid points), for the analysis in this thesis, the data points are the reference points. $h$, which determines the level of smoothing of the estimated density, is the RMS width of the kernel function, typically accompanied by some data-dependent factor (e.g., covariance matrix). Unlike the histogram estimator, where the choice of bin width affects both the level of smoothing and the number of data points in the bin (occasionally some bins are left empty), the choice of kernel width only affects the level of smoothing of the estimated density. In this thesis, the bandwidth selection rule known as Scott's Normal reference rule is used (more on this below).

In general, a kernel function should satisfy a particular set of conditions for the estimated density to satisfy the requirements of a PDF [45]: 
1. Non-negative: $k(x) \geqslant 0$.

2. Integrates to $1: \int_{-\infty}^{\infty} k(x) d x=1$.

3. Symmetric about its mean value: $\int_{-\infty}^{\infty} x k(x) d x=0$.

4. Satisfies finite second moment condition: $k_{2}(k)=\int_{-\infty}^{\infty} x^{2} k(x) d x>0$.

Condition 2 ensures that the kernel function is normalized to unity and that the resulting estimated density is a PDF; this is indeed the case as illustrated below:

$$
\begin{gathered}
\int_{-\infty}^{\infty} \hat{f}(x) d x=\int_{-\infty}^{\infty} \frac{1}{n} \sum_{i=1}^{n} \frac{1}{h} k\left(\frac{x-X_{i}}{h}\right) d x=\frac{1}{n} \sum_{i=1}^{n} \int_{-\infty}^{\infty} \frac{1}{h} k\left(\frac{x-X_{i}}{h}\right) d x= \\
\frac{1}{n} \sum_{i=1}^{n} \int_{-\infty}^{\infty} k(u) d u=\frac{1}{n} \sum_{i=1}^{n} 1=\frac{n}{n}=1
\end{gathered}
$$

where the substitution variable, $u$ is equal to $\frac{x-X_{i}}{h}$.

Condition $4, k_{j}(k)=\int x^{j} k(x) d x$ defines the moment integral of the kernel function. The first non-zero moment indicates the order of the kernel. For instance, if the first non-zero moment of the kernel function is the second, then the kernel is of second order. In this thesis, second-order, $j=2$ (signifies symmetric and nonnegative) Gaussian kernel functions are used. The symmetry condition, Condition 3 is necessary to ensure that the kernel function is centered about $X_{i}$.

One can obtain the moments of the estimated distribution using Conditions 1-4 above. The first moment of the estimated PDF using Condition 2 would lead to the sample mean [46]:

$$
\begin{aligned}
\int_{-\infty}^{\infty} x \hat{f}(x) d x=\frac{1}{n} & \sum_{i=1}^{n} \int_{-\infty}^{\infty} x \frac{1}{h} k\left(\frac{x-X_{i}}{h}\right) d x=\frac{1}{n} \sum_{i=1}^{n} \int_{-\infty}^{\infty}\left(X_{i}+u h\right) k(u) d u= \\
& \frac{1}{n} \sum_{i=1}^{n} X_{i} \int_{-\infty}^{\infty} k(u) d u+\frac{1}{n} \sum_{i=1}^{n} h \int_{-\infty}^{\infty} u k(u) d u=\frac{1}{n} \sum_{i=1}^{n} X_{i} .
\end{aligned}
$$


The second moment of the estimated PDF using Condition 4 would lead to a description of the sample variance [46],

$$
\begin{array}{r}
\int_{-\infty}^{\infty} x^{2} \hat{f}(x) d x=\frac{1}{n} \sum_{i=1}^{n} \int_{-\infty}^{\infty} x^{2} \frac{1}{h} k\left(\frac{x-X_{i}}{h}\right) d x=\frac{1}{n} \sum_{i=1}^{n} \int_{-\infty}^{\infty}\left(X_{i}+u h\right)^{2} k(u) d u= \\
\frac{1}{n} \sum_{i=1}^{n} X_{i}^{2}+\frac{2}{n} \sum_{i=1}^{n} X_{i} h \int_{-\infty}^{\infty} u k(u) d u+\frac{1}{n} \sum_{i=1}^{n} h^{2} \int_{-\infty}^{\infty} u^{2} k(u) d u= \\
\frac{1}{n} \sum_{i=1}^{n} X_{i}^{2}+\frac{1}{n} \sum_{i=1}^{n} h^{2} k_{2}(k),
\end{array}
$$

where sample variance, given by the relation $\frac{1}{n} \sum_{i=1}^{n} X_{i}^{2}$, is inflated by the second moment kernel factor $\frac{1}{n} \sum_{i=1}^{n} h^{2} k_{2}(k)$. This is a general characteristic of all density estimation methods where the estimated density is a smoothed version of the true PDF plus an extra factor of $\frac{1}{n} \sum_{i=1}^{n} h^{2} k_{2}(k)$ in the sample variance [45].

In density estimation analysis, the goal is to ensure that the discrepancy between the estimated PDF and the true PDF is small; this is typically measured using the mean square error, MSE term,

$$
\operatorname{MSE}(\hat{f}(x))=\mathrm{E}(\hat{f}(x)-f(x))^{2},
$$

where "E" is the expectated value operator; an example is the expectation of the estimated PDF, $E \hat{f}(x)[45,47]$,

$$
E \hat{f}(x)=\frac{1}{n} \sum_{i=1}^{n} E \frac{1}{h} k\left(\frac{x-X_{i}}{h}\right)=\int_{-\infty}^{\infty} \frac{1}{h} k\left(\frac{x-z}{h}\right) f(z) d z,
$$

As shown in Equation 3.14, the estimated PDF is the convolution of the true PDF, $f$ with the kernel function, $k\left(\frac{x-z}{h}\right)$. This convolution process smoothes the estimated density curve. Hence, for a realistic representation of the true density, the smoothed estimated density curve should be given some noise; this was introduced as the term $\frac{1}{n} \sum_{i=1}^{n} h^{2} k_{2}(k)$ in the sample variance (Eq. 3.12). In this context, Equation 3.13 can be rewritten in terms of the smoothness, or the so-called estimator bias squared, and 
the noise or the so-called estimator variance (not to be confused with sample and distribution variance) terms [45, 47]:

$$
\begin{array}{r}
\operatorname{MSE}(\hat{f}(x))=\mathrm{E}(\hat{f}-f)^{2}= \\
\mathrm{E}\left[(\hat{f}-\mathrm{E}(\hat{f})+\mathrm{E}(\hat{f})-f)^{2}\right]= \\
\mathrm{E}\left[(\hat{f}-\mathrm{E}(\hat{f}))^{2}+2(\hat{f}-\mathrm{E}(\hat{f}))(\mathrm{E}(\hat{f})-f)+(\mathrm{E}(\hat{f})-f)^{2}\right]= \\
\mathrm{E}\left[(\hat{f}-\mathrm{E}(\hat{f}))^{2}\right]+\mathrm{E}[2(\hat{f}-\mathrm{E}(\hat{f}))(\mathrm{E}(\hat{f})-f)]+\mathrm{E}\left[(\mathrm{E}(\hat{f})-f)^{2}\right]= \\
\mathrm{E}\left[(\hat{f}-\mathrm{E}(\hat{f}))^{2}\right]+2(\mathrm{E}(\hat{f}-f)) \mathrm{E}[\hat{f}-\mathrm{E}(\hat{f})]+(\mathrm{E}(\hat{f})-f)^{2}= \\
\mathrm{E}\left[(\hat{f}-\mathrm{E}(\hat{f}))^{2}\right]+2(\mathrm{E}(\hat{f}-f))(\mathrm{E}(\hat{f})-\mathrm{E}(\hat{f}))+(\mathrm{E}(\hat{f})-f)^{2}= \\
\mathrm{E}\left[(\hat{f}-\mathrm{E}(\hat{f}))^{2}\right]+(\mathrm{E}(\hat{f})-f)^{2}= \\
{[E \hat{f}(x)-f(x)]+\left[E\left(\hat{f}^{2}(x)\right)-(E \hat{f}(x))^{2}\right]=} \\
{[\operatorname{bias}(\hat{f})]^{2}+\operatorname{variance}(\hat{f}),}
\end{array}
$$

where, for simplicity, $\hat{f}(x)$ and $f(x)$ notations were replaced by $\hat{f}$ and $f$. To derive the bias and variance terms in Equation 3.15, it was assumed that $\mathrm{E}(\hat{f})-f$ and $\mathrm{E}(\hat{f})$ were constant.

To reiterate, the bias term in Equation 3.15 is the error associated with the level of smoothing in the estimated density; in a sense, it represents the measure of the accuracy of the estimated density [45],

$$
\operatorname{bias}(\hat{f}(x))=[E \hat{f}(x)-f(x)]
$$

A large bias in the estimated density means that the estimated density is overly smooth and the measurement may thus not be accurate. Variance, on the other hand is the error associated with the level of noise in the estimated density curve and is the measure of the precision of the estimated density [45],

$$
\operatorname{variance}(\hat{f}(x))=\left[E\left(\hat{f}^{2}(x)\right)-(E \hat{f}(x))^{2}\right] \text {. }
$$


If the estimated density has a large variance, it means that the estimated density is overly noisy or spiky and not precise.

The parameter that controls the bias and variance is the kernel width, commonly known as bandwidth parameter, $h$. Finding a balance between bias and variance is important in non-parametric density estimation, and the bandwidth parameter should be optimized to keep such a balance and minimize MSE. As shown in Equation 3.15, the bias and variance terms contain the unknown true PDF term, $f(x) .{ }^{25}$ Therefore, to solve for the optimal bandwidth parameter using the bias and variance terms (and the MSE term), an assumption of the true PDF should be made, and asymptotic approximations (in the limit where $n \rightarrow \infty$ ) should be used. In the case of the MICE muon beam, since the beam is nearly Gaussian, the true PDF is assumed to be Gaussian. Making such an assumption, however, is not the same as assuming a Gaussian and fitting a Gaussian to the entire distribution to estimate the distribution mean and variance (as is done in the parametric density estimation approach). The assumption used in determining the bandwidth parameter affects the width of the kernel function; the kernels are still centered at each data point, and each data point still counts toward estimating the underlying density. The paragraphs that follow aim at deriving the optimal bandwidth parameter.

The MSE term described in Equation 3.13 measures the error of the estimated PDF at a single point $x$. A more global measure of MSE is referred to as the mean integrated square error, MISE. The MISE can be written as the integrated bias and

${ }^{25}$ As a reminder, non-parametric density estimators, are used when the true $\mathrm{PDF}$ is not known 
variance terms [45]:

$$
\begin{array}{r}
\operatorname{MiSE}(\hat{f}(x))= \\
\int_{-\infty}^{\infty}[\operatorname{bias}(\hat{f}(x))]^{2} d x+\int_{-\infty}^{\infty} \operatorname{variance}(\hat{f}(x)) d x= \\
\int_{-\infty}^{\infty}[E \hat{f}(x)-f(x)]^{2} d x+\int_{-\infty}^{\infty}\left[E\left(\hat{f}^{2}(x)\right)-(E \hat{f}(x))^{2}\right] d x
\end{array}
$$

where the bias squared and variance terms can be rewritten as

$$
\begin{aligned}
{[\operatorname{bias}(\hat{f}(x))]^{2} } & =\left(\int_{-\infty}^{\infty} \frac{1}{h} k\left(\frac{x-z}{h}\right) f(z) d z-f(x)\right)^{2} \\
\operatorname{variance}(\hat{f}(x)) & =\frac{1}{n} \int_{-\infty}^{\infty} \frac{1}{h^{2}} k\left(\frac{x-z}{h}\right)^{2} f(z) d z-\frac{1}{n}\left(\frac{1}{h} \int_{-\infty}^{\infty} k\left(\frac{x-z}{h}\right) f(z) d z\right)^{2} .
\end{aligned}
$$

The MISE term above cannot be solved for unless the true $\operatorname{PDF} f(z)$ is known; hence a functional form for $f(z)$ should be assumed. This assumption comes later when the optimal bandwidth parameter is determined. In the meantime, asymptotic approximations (the limit in which $n \rightarrow \infty$ or $h \rightarrow 0$ which leads to $\hat{f} \rightarrow f$ [45]) can be applied to solve for the asymptotic MISE or AMISE (same mathematical form as Eq. 3.19). The starting point would be to obtain the asymptotic bias term (from Eq. 3.16). A change of variable, $z=x+h u$ in the bias term from Equation 3.19 leads to [45]:

$$
\operatorname{bias}(\hat{f}(x))=\int_{-\infty}^{\infty} k(u) f(x+h u) d u-f(x)
$$

It is possible to Taylor expand $f(x+h u)$ [45],

$$
f(x+h u)=f(x)+f^{\prime}(x) h u+\frac{1}{2 !} f^{\prime \prime}(x) h^{2} u^{2}+\frac{1}{3 !} f^{(3)}(x) h^{3} u^{3}+\ldots
$$

Substituting the Taylor expanded term into the first term of Equation 3.20 and im- 
posing Conditions 3 and 4 leads to [45]:

$$
\begin{array}{r}
\operatorname{bias}(\hat{f}(x))= \\
\int_{-\infty}^{\infty} k(u) f(x+u h) d u-f(x)= \\
\left.f^{\prime}(x) h k_{1}(k)+\frac{1}{2} f^{\prime \prime}(x) h^{2} k_{2}(k)+\frac{1}{3 !} f^{(3)}(x) h^{3} k_{3}(k)+\ldots\right)= \\
\frac{1}{2 !} f^{\prime \prime}(x) h^{2} k_{2}(k)+O\left(h^{3}\right) \ldots
\end{array}
$$

According to Equation 3.22, for kernels of the second order (kernels satisfying Condition 4), the bias depends on the square of the bandwidth parameter, $h^{2}$; hence, the larger the bandwidth, the greater the bias of the estimated PDF. In other words, a large bandwidth parameter leads to a smooth estimated PDF. Integrating the bias term in Equation 3.22 over the entire $x$ space and taking its square leads to [45]:

$$
\begin{gathered}
{\left[\int_{-\infty}^{\infty} \operatorname{bias}(\hat{f}(x) d x)\right]^{2}=} \\
\left(\int_{-\infty}^{\infty} \frac{1}{h} k\left(\frac{x-z}{h}\right) f(z) d z-f(x)\right)^{2} \approx \\
\frac{1}{4} h^{4} k_{2}^{2}(k) \int_{-\infty}^{\infty} f^{\prime \prime}(x)^{2} d x
\end{gathered}
$$

The variance term on the other hand takes the following form [45]:

$$
\begin{array}{r}
\operatorname{variance}(\hat{f}(x))=\frac{1}{n} \int_{-\infty}^{\infty} \frac{1}{h^{2}} k\left(\frac{x-z}{h}\right)^{2} f(z) d z-\frac{1}{n}(f(x)+\operatorname{bias}(\hat{f}(x)))^{2} \\
\approx \frac{1}{n} \int_{-\infty}^{\infty} \frac{1}{h} f(x+h u) k^{2}(u) d u-\frac{1}{n}\left(f(x)+\mathrm{O}\left(h^{2}\right)\right)^{2}
\end{array}
$$

where the second integral in Equation 3.24 is rewritten as the bias term. Substituting the Taylor expanded term from Equation 3.21 into Equation 3.24, followed by the assumption that $h$ approaches zero as $n$ approaches infinity (the asymptotic approximation where the limit of the estimated PDF approaches the true PDF) leads to the following variance term [45]:

$$
\operatorname{variance}(\hat{f}(x))=\frac{1}{n} \frac{1}{h} f(x) \int_{-\infty}^{\infty} k^{2}(u) d u+\mathrm{O}\left(\frac{1}{n}\right) \approx \frac{1}{n} \frac{1}{h} f(x) \int_{-\infty}^{\infty} k^{2}(u) d u
$$


Integrating the variance over the entire $x$ space leads to [45]

$$
\int_{-\infty}^{\infty} \operatorname{variance}(\hat{f}(x)) d x=\frac{1}{n} \frac{1}{h} \int_{-\infty}^{\infty} k^{2}(u) d u .
$$

According to Equation 3.26, the variance depends inversely on the bandwidth parameter, $h$; hence, the smaller the bandwidth parameter, the greater the variance. A small bandwidth parameter leads to a noisy estimated PDF. Combined with the definition of bias in Equation 3.22, it can be observed that the choice of bandwidth parameter creates a trade-off between smoothness and the noise in the estimated density curve; this is a known feature in the non-parametric density estimation methods, and the bandwidth parameter derived below minimizes this effect for a nearly Gaussian distribution (e.g., the assumed distribution for the MICE muon beam).

Substituting the bias squared term from Equation 3.23 and the variance term from Equation 3.26 into Equation 3.19 leads to the AMISE term below [45]:

$$
\begin{aligned}
\operatorname{AMiSE}(\hat{f}(x)) & =\left(\frac{1}{2} f^{\prime \prime}(x) h^{2} u^{2} k_{2}(k)\right)^{2}+\frac{1}{n} \frac{1}{h} f(x) \int_{-\infty}^{\infty} k(u)^{2} d u \\
& =\frac{1}{4} h^{4} k_{2}^{2}(k) \int_{-\infty}^{\infty} f^{\prime \prime}(x)^{2} d x \frac{1}{n} \frac{1}{h} \int_{-\infty}^{\infty} k(u)^{2} d u
\end{aligned}
$$

As pointed out above, Equation 3.27 demonstrates a bias-variance trade-off: reducing the bias by choosing a small bandwidth parameter, $h$ leads to a large variance and vice versa, as illustrated in Figure 3.3. To produce the density curves in Figure 3.3, the kernel density estimator is applied to the $x$ coordinates of a sub-sample of 500 muons at the entrance of the MICE upstream tracker; the distribution is not known a priori since the data points are measured muons, hence the use of a nonparametric density estimator to estimate the distribution. Section 3.3 below covers the KDE validation against a known distribution (e.g., random sample drawn from a Gaussian distribution).

The optimal bandwidth parameter, often referred to as the asymptotically optimal bandwidth, is found by minimizing the Asymptotic MISE or AMISE [45]; for 


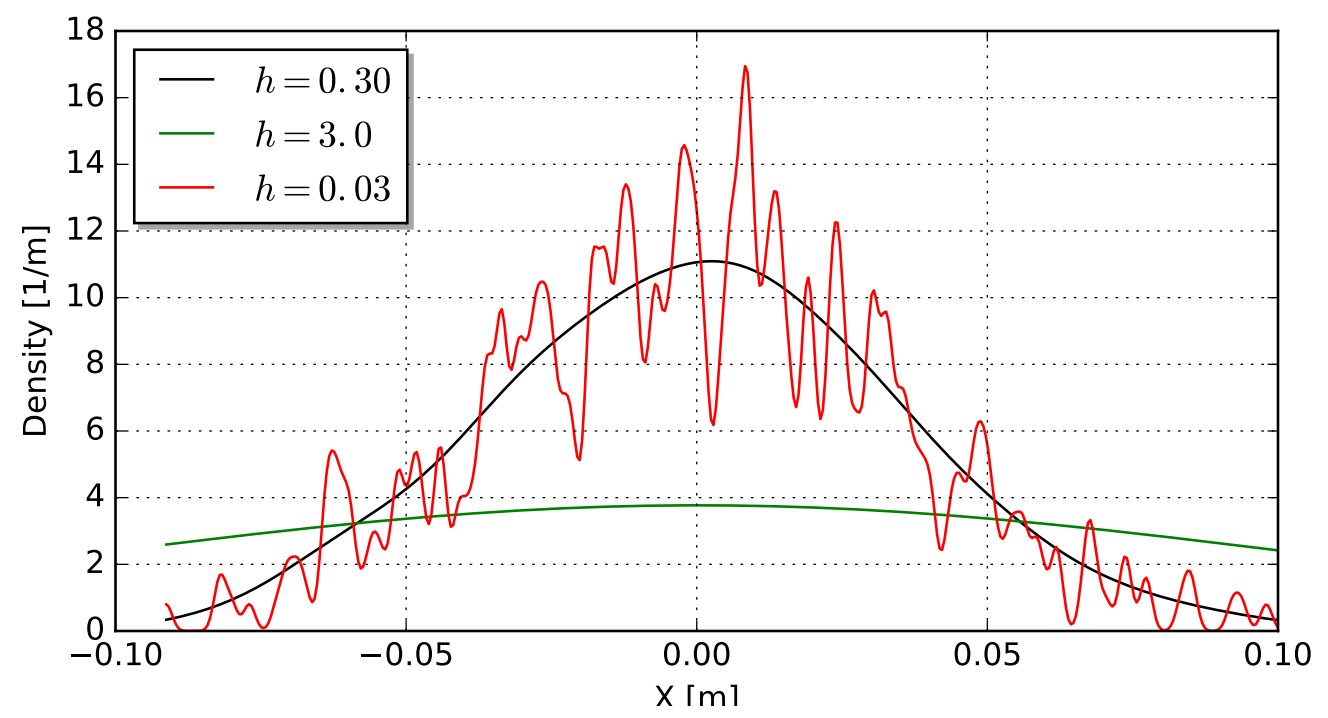

Figure 3.3. The $x$ distribution is approximately Gaussian with the optimal bandwidth parameter, $h=0.3$. When the bandwidth parameter is 3.0, the estimated PDF is oversmoothed (green curve). A smaller value, 0.03, at the bandwidth parameter leads to a noisier estimated density (red curve) [48].

a second order kernel function in one dimension, it is [45]

$$
\begin{aligned}
\frac{d}{d h} \operatorname{AMISE}(\hat{f}(x)) & \approx \frac{d}{d h}\left(\frac{1}{4} h^{4} k_{2}^{2}(k) \int_{-\infty}^{\infty} f^{\prime \prime 2}(x) d x+\frac{1}{n} \frac{1}{h} \int_{-\infty}^{\infty} k^{2}(u) d u\right)=0 \\
h_{\text {optimal }} & =k_{2}(k)^{-\frac{2}{5}}\left(\int_{-\infty}^{\infty} k^{2}(u) d u\right)^{\frac{1}{5}}\left(\int_{-\infty}^{\infty} f^{\prime \prime 2}(x) d x\right)^{-\frac{1}{5}} n^{-\frac{1}{5}}
\end{aligned}
$$

Equation 3.29 illustrates that the ideal bandwidth parameter approaches zero with increasing sample size [45]; this is the asymptotic approximation with which the AMISE term was derived. The $\int_{-\infty}^{\infty}\left(f^{\prime \prime}(x)\right)^{2} d x$ term represents the rate of fluctuation of the true density. One can observe that smaller values of $h$ are optimal for a rapidly fluctuating density curve. In general (as mentioned above), smaller $h$ values are more appropriate for a rapidly fluctuating (or noisy) density curve while larger $h$ values are more suited for a smoother density curve; Figure 3.4 illustrates this. What is referred to as "true" density curve in Figure 3.4 refers to a random sample size of 10,000 drawn from a Gaussian distribution and is produced using the probability density 


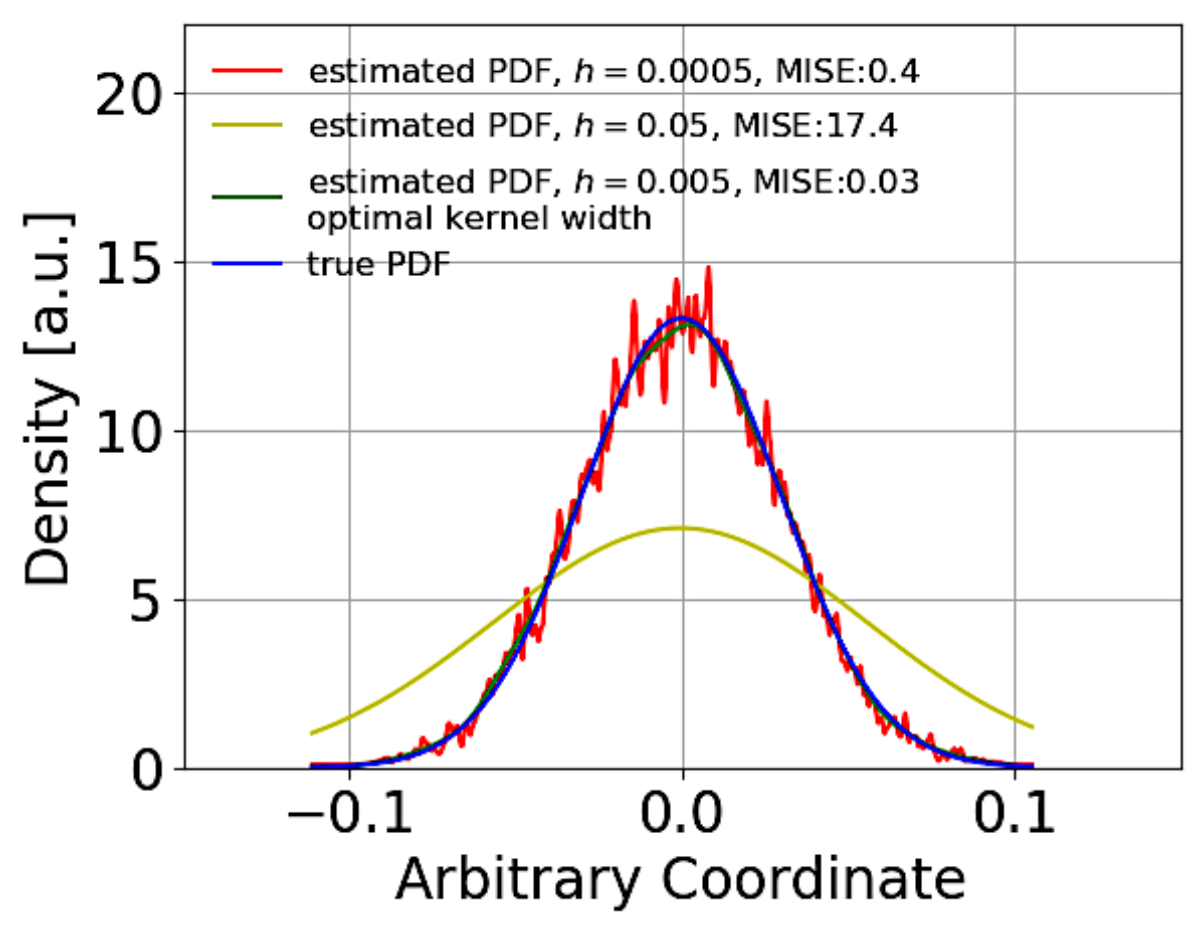

Figure 3.4. The "true" distribution is randomly drawn from a Gaussian. As expected, green curve has minimized mean integrated square error [48].

equation for a Gaussian distribution function,

$$
f(\vec{x})=\frac{\left|\sigma^{-1 / 2}\right|}{\sqrt{2 \pi^{d}}} \exp \left(\frac{-(\vec{x}-\vec{m})^{T} \sigma^{-1}(\vec{x}-\vec{m})}{2}\right) .
$$

where $\vec{m}$ is the distribution mean and $\sigma$ the standard deviation. As expected, Figure 3.4 illustrates that the estimated density curve obtained using the optimal bandwidth parameter, $h=0.005$ (green curve), represents the "true" density curve best, and as a result, has the smallest MISE (more on optimal bandwidth parameter in Section 3.2.1). The section that follows covers the process of obtaining a value for the optimal bandwidth parameter, similar to the optimal bandwidth parameter used in producing Figure 3.4.

3.2.1 Univariate Kernel Density Estimation. The unknown fluctuation term $\int_{-\infty}^{\infty}\left(f^{\prime \prime}(x)\right)^{2} d x$ is due to the unknown true density term $\left.f(x)\right)$ from Equation 3.29. This term can be solved if a functional form for the distribution is assumed (e.g., Gaussians). In particular, the standard normal distribution (Gaussian distribution 
with mean of zero) can be used. Determining the bandwidth parameter using the standard normal distribution is referred to as the "normal reference rule" (also known as the rule-of-thumb or the plug-in method $[45,47])$, since there is a reference to the normal distribution. With $\phi(x)$ as a new notation for the true PDF, $f(x), \sigma^{2}$ as the variance, and a distribution mean of zero, one can solve for the fluctuation term, $\int_{-\infty}^{\infty}\left(f^{\prime \prime}(x)\right)^{2} d x:$

$$
\int_{-\infty}^{\infty} f^{\prime \prime}(x) d x=\sigma^{-5} \int_{-\infty}^{\infty} \phi^{\prime \prime}(x)^{2} d x=\frac{3}{8} \pi^{-1 / 2} \sigma^{-5} \approx 0.212 \sigma^{-5}
$$

To solve for the optimal bandwidth parameter, one should substitute Equation 3.31 and the Gaussian kernel function into Equation 3.29 from the previous section. The optimal bandwidth parameter in one dimension would then be [45]

$$
h_{\text {optimal }}=(4 \pi)^{-1 / 10}\left(\frac{3}{8} \pi^{-1 / 2}\right)^{-1 / 5} \sigma n^{-1 / 5}=\left(\frac{4}{3}\right)^{\frac{1}{5}} \sigma n^{-1 / 5}=1.06 \sigma n^{-1 / 5} .
$$

3.2.2 Multivariate Kernel Density Estimation. The six-dimensional positionmomentum and energy-time coordinates are used to represent individual muons in phase space. Muon position and momentum values $\vec{X}_{i}=\left(x_{i}, p_{x_{i}}, y_{i}, p_{y_{i}}\right)$ are reconstructed one by one in the MICE tracking detectors [42], where $i$ runs from 1 to $n$ and $n$ represents the total number of muons in the sample under study. The kernel density estimator can then be applied in four dimensions to the muons in the MICE beam. Similarly to the previous section, to find $h_{\text {optimal }}$ in multiple dimensions (in the four or six-dimensional phase space used to describe each muon in the MICE beam), the AMISE needs to be minimized. The starting point is to obtain the $d$-dimensional bias and variance terms. Following a set of steps similar to the previous section, the multi-dimensional bias and variance terms become [45, 47]:

$$
\begin{gathered}
\operatorname{bias}(\hat{f}(\vec{x})) \approx \frac{1}{2} h^{2} k_{2}(k) \nabla^{2} f(\vec{x}), \\
\operatorname{variance}(\hat{f}(\vec{x})) \approx n^{-1} h^{-d} f(\vec{x}) \int_{-\infty}^{\infty}(k(u))^{2} d u,
\end{gathered}
$$


where $k(u)$ is the kernel function, $u$ the substitution variable $u=\frac{x-z}{h}$, and $\nabla^{2}$ the Laplacian operator.

Taking the integral of Equations 3.33 and 3.34 leads to the multi-dimensional AMISE term:

$$
\operatorname{AMiSE}(\hat{f}(\vec{x})) \approx \frac{1}{4} h^{4} k_{2}(k) \int_{-\infty}^{\infty}\left(\nabla^{2} f(\vec{x})\right)^{2} d x+n^{-1} h^{-d} \int_{-\infty}^{\infty}(k(u))^{2} d u .
$$

Minimizing Equation 3.35 leads to the optimal bandwidth parameter in multiple dimensions [45],

$$
\begin{gathered}
h_{\text {optimal }}=\left(d R(k) \overrightarrow{k_{2}}(k)^{-2}\right)^{\frac{1}{d+4}}\left(\int_{-\infty}^{\infty}\left(\nabla^{2} f(\vec{x})\right)^{2} d x\right)^{\frac{-1}{d+4}} n^{\frac{-1}{d+4}} . \\
h_{\text {optimal }}=\left(\frac{4}{d+4}\right)^{-\frac{1}{d+4}} \Sigma n^{-\frac{1}{d+4}}=h_{\text {factor }} \sum n^{-\frac{1}{d+4}},
\end{gathered}
$$

where $R(k)=\int_{-\infty}^{\infty}(k(u))^{2} d u, \overrightarrow{k_{2}}(k)=\int_{-\infty}^{\infty} u^{2} k(u) d u$, and $\Sigma$ is the covariance matrix of the dataset. The bandwidth parameter from Equation 3.37 is used with $h_{\text {factor }}$ selected to be 1 following Scott's normal reference rule (normal reference rule because there is a reference to Normal distribution) $[45,47]$. The dimension variable $d$ can be 1 and higher; in MICE, it is the dimension of the phase space (4 or 6). The estimated multi-dimensional (multivariate) density using the KDE technique is then

$$
\hat{f}(\vec{x})=\frac{|\Sigma|^{-1 / 2}}{n h_{\text {factor }}^{d} \sqrt{(2 \pi)^{d}}} \sum_{i=1}^{n} \exp \left[-\frac{\left(\vec{x}-\vec{X}_{i}\right)^{T} \Sigma^{-1}\left(\vec{x}-\vec{X}_{i}\right)}{2 h_{\text {factor }}^{2}}\right] .
$$

\subsection{Validation against Known Distributions}

Here, the impact of sample size and bandwidth parameter on the performance of the KDE technique for a known PDF is studied. In particular, the univariate Gaussian distributions of known means and standard deviations were used; in each study, Monte Carlo (MC) simulation was used to draw 1000, 10,000, and 100,000 random numbers from a Gaussian distribution 10 times (10 trials for each sample 
size). The kernel density estimator (Eq. 3.7) and the true Gaussian density relation (Eq. 3.39) were applied to each of these generated samples. The true univariate Gaussian PDF is similar to the univariate form of the KDE relation from Equation 3.7. The only difference is that in the Gaussian density equation, the sample mean, $\vec{m}$, replaces the coordinate of the $i^{\text {th }}$ data point in the sample, $\vec{X}_{i}$ :

$$
f(\vec{x})=\frac{\left|\Sigma^{-1 / 2}\right|}{\sqrt{(2 \pi)^{d}}} \exp \left(\frac{-(\vec{x}-\vec{m})^{T} \Sigma^{-1}(\vec{x}-\vec{m})}{2}\right) .
$$

In Equation 3.39, $\Sigma$ in the multi-dimensional phase space is the covariance matrix, $m$ the sample mean, $\vec{x}$ the coordinate at which the density is calculated (reference point), and $d$ the dimensionality of the dataset ( $d=1$ for the plots presented in this section). The variables $\left(x, p_{x}, y, p_{y}\right)$ used in the KDE validation study are selected to have the same variances as the corresponding coordinates of the muons in the MICE beam at the entrance to the upstream tracker $\left(\sigma_{x}=\sigma_{y}=0.03 \mathrm{~m}\right.$ and $\left.\sigma_{p x}=\sigma_{p y}=0.02 \mathrm{GeV} / c\right)$. The mean of each variable is selected to be zero. As an illustration, the deviation of the KDE estimated density from the true density in $x$ (1D) for the different sample sizes of 1000, 10,000, and 100, 000 is shown in Figures 3.5, 3.6, and 3.7. The left-hand side plots in Figures 3.5, 3.6, and 3.7 illustrate the 10 random samples drawn from the same distribution: "KDE density" (KDE here is an acronym for kernel density estimator) is the estimated density using the KDE technique, and "true density" is calculated using the Gaussian relation in Equation 3.39. The upper-left plots in Figures 3.5, 3.6, and 3.7 compare the 10 different KDE density curves with the 10 different true density curves. The lower-left plots in Figures 3.5, 3.6, and 3.7 compare the average KDE density curve (averaged over the 10 different measurements) with the average true density curve. The right-hand side plots in Figures 3.5, 3.6, and 3.7, on the other hand, illustrate the differences between the estimated density using the KDE technique and the true density curve(s). A comparison of the distribution of larger sample sizes (such as the one shown in Fig. 3.7) with the distribution of smaller sample sizes (such as the one shown in Fig. 3.5) reveals that the discrepancy 

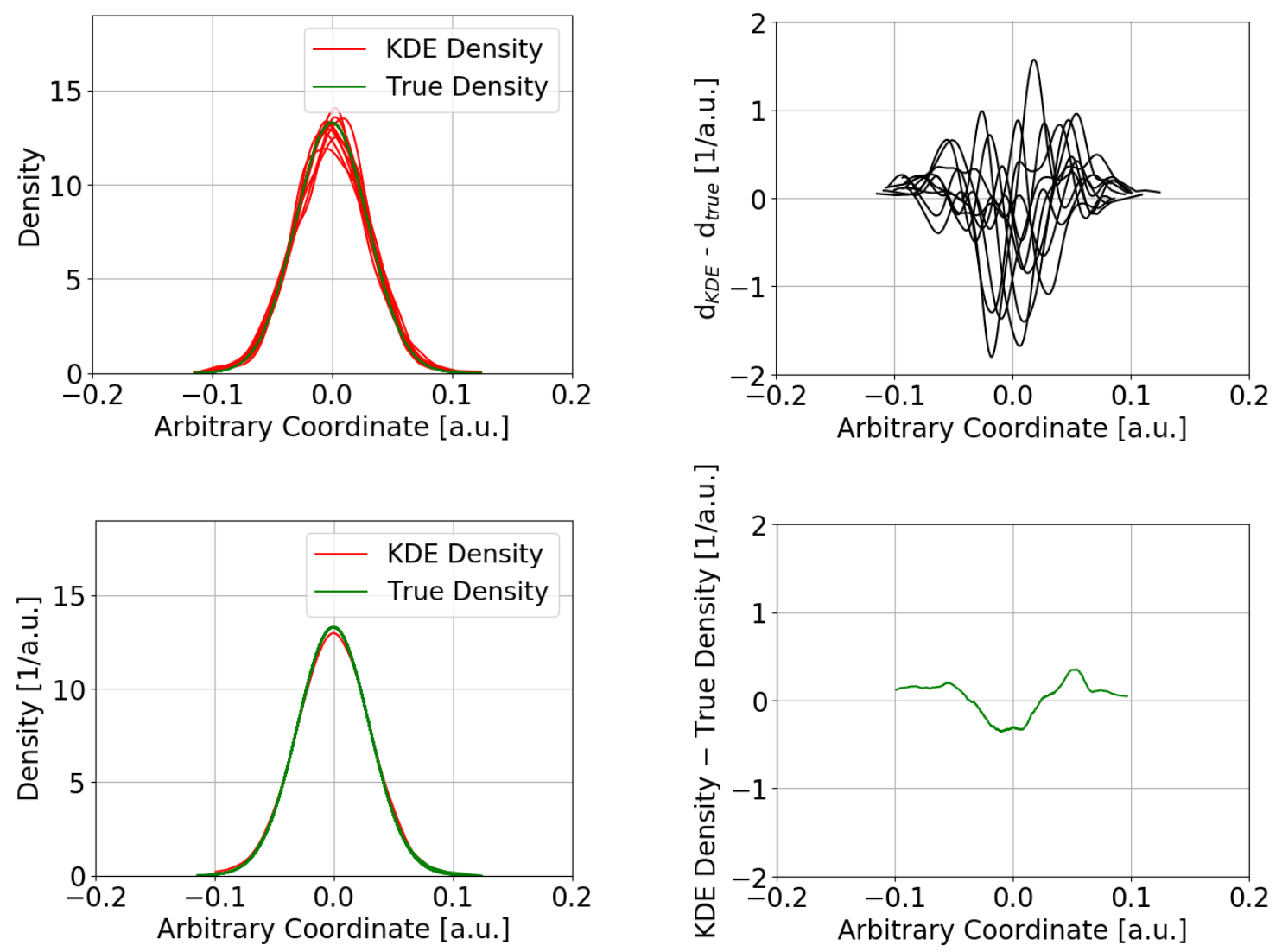

Figure 3.5. The true and KDE $x$ distributions containing 1000 data points. The data points are drawn from a Gaussian distribution 10 times. The red curve(s) are the KDE density curve(s) while the green curve(s) are the true $\mathrm{PDF}(\mathrm{s})$. The left-hand side plots are the 10 random samples of the true density and the KDE density, while the right-hand side plots are their averages. 

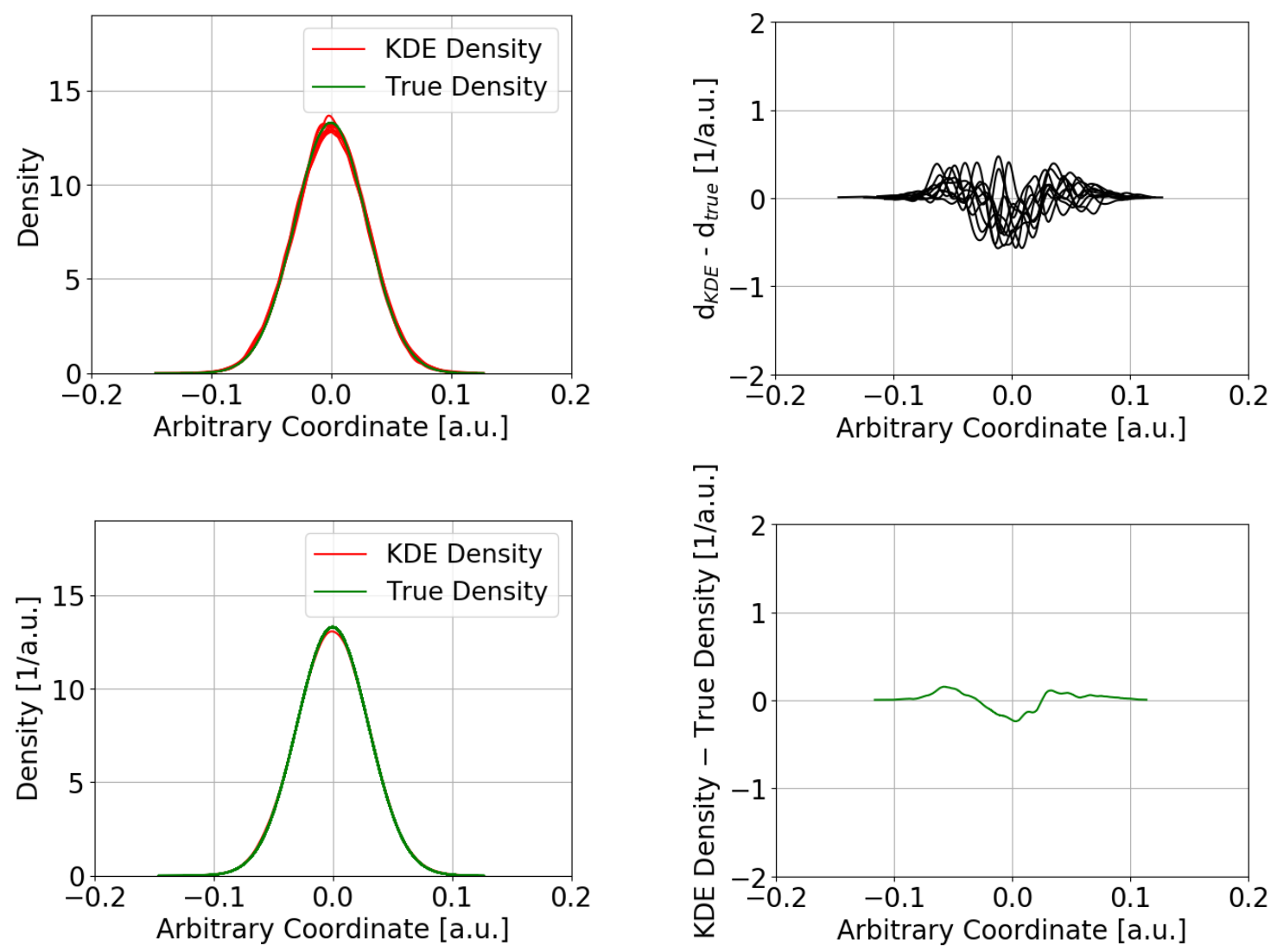

Figure 3.6. The true and KDE $x$ distributions containing 10,000 data points. The data points are drawn from a Gaussian distribution 10 times. The red curve(s) are the KDE density curve(s) while the green curve(s) are the true PDF(s). The left-hand side plots are the 10 random samples of the true density and the KDE density, while the right-hand side plots are their averages. 

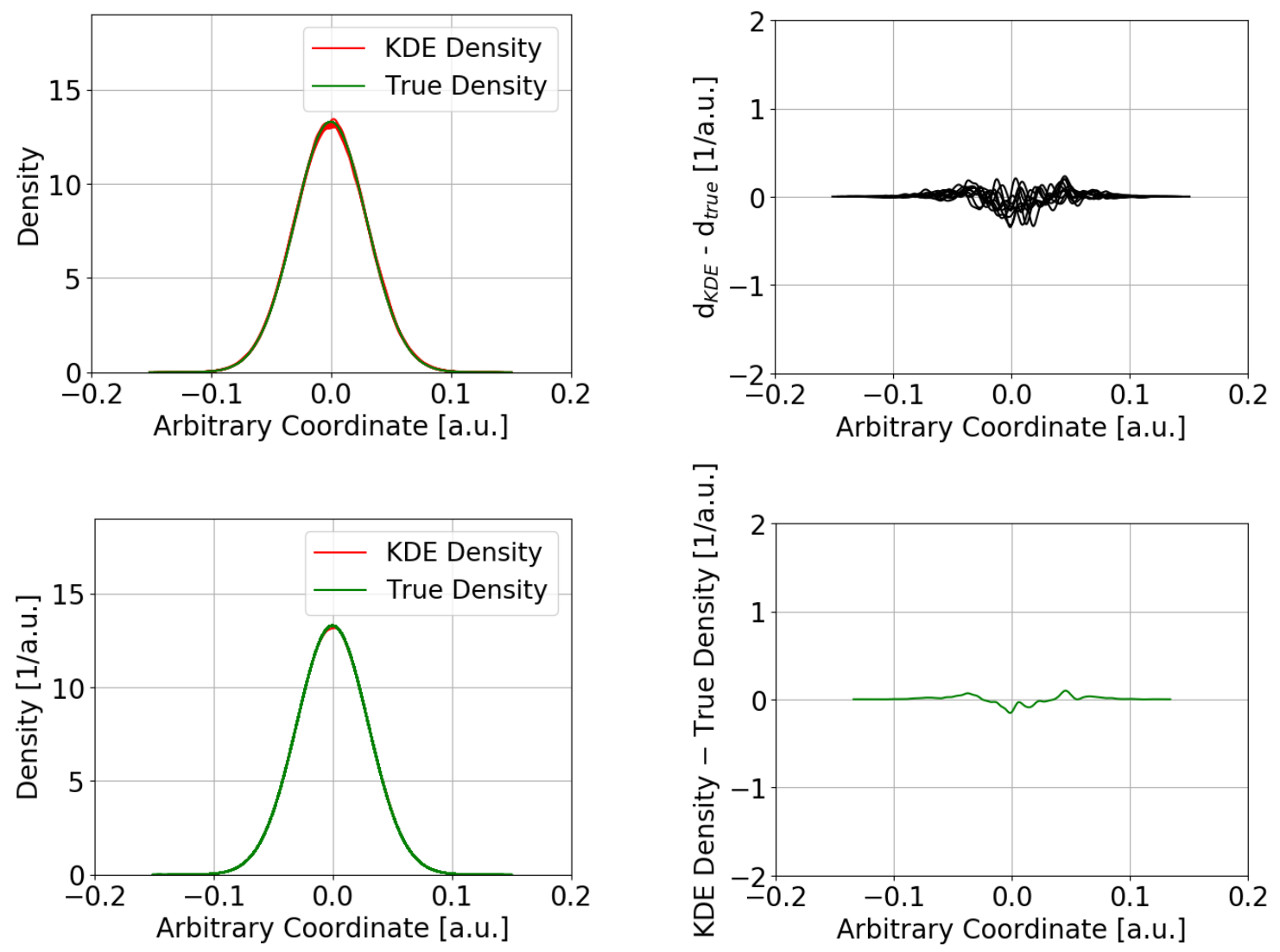

Figure 3.7. The true and KDE $x$ distributions containing 100,000 data points. The data points are drawn from a Gaussian distribution 10 times. The red curve(s) are the KDE density curve(s) while the green curve(s) are the true $\operatorname{PDF}(\mathrm{s})$. The left-hand side plots are the 10 random samples of the true density and the KDE density, while the right-hand side plots are their averages.

between KDE and the true density becomes progressively smaller for larger samples. As shown in Figures 3.5 through 3.7, the estimated density tends to be larger than the true density in the tail of the distribution and smaller than the true density at the core of the distribution. This effect is more apparent for smaller sample sizes than for larger samples, indicating that the density estimated using the KDE technique converges to the true density, $\hat{f} \rightarrow f$ as $n \rightarrow \infty$. This convergence to the true density is also observed in the lower left-hand plots in Figures 3.5, 3.6, and 3.7, where the 10 estimated density curves in red converge to the true density in green as sample size grows from 1000 to 100,000 . 


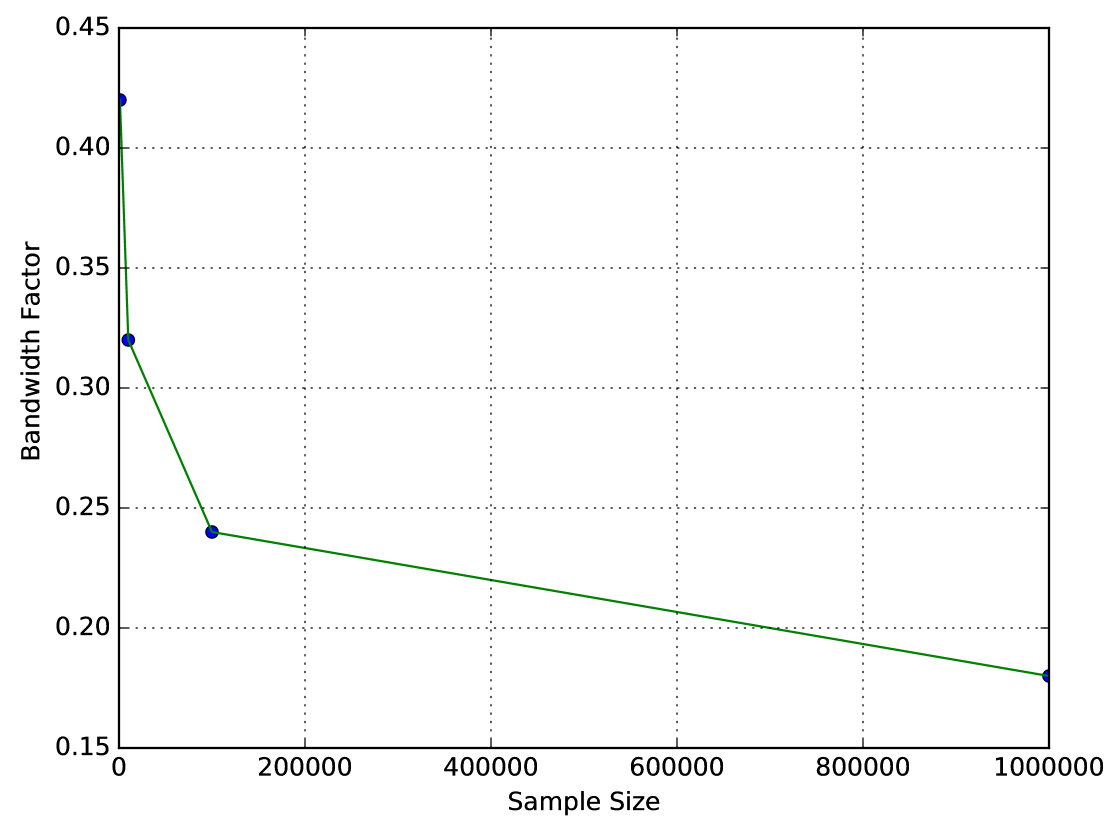

Figure 3.8. The variation of the bandwidth factorr, $h_{\text {factor }}$ with the sample size.

How the estimated density changes with variations in the bandwidth parameter depends on how the bandwidth parameter is defined. The bandwidth parameter used in the muon beam cooling studies in MICE is Scott's normal reference rule [45]. This bandwidth parameter was derived in Section 3.2 and its univariate and multivariate forms are defined in Equations 3.32 and 3.37. In both of these equations, the bandwidth parameter depends on the sample size as well as the dimensionality of the dataset. The dependence of the bandwidth parameter on the sample size is illustrated in Figures 3.8 and 3.9. Figure 3.8 shows that as the sample size grows, the optimal bandwidth parameter decreases; this is because as the sample size grows, the core, as well as the tail of the distribution, becomes more populated. To compensate for the closer proximity of the data points, the bandwidth parameter should decrease; otherwise, the detailed features in the distribution would be obscured. The same applies for smaller sample sizes: as shown in Figure 3.9, as the sample size decreases, the occupancy of the data points becomes smaller, and the bandwidth parameter must become large to ensure that the bandwidth does not overemphasize noise. Fig- 


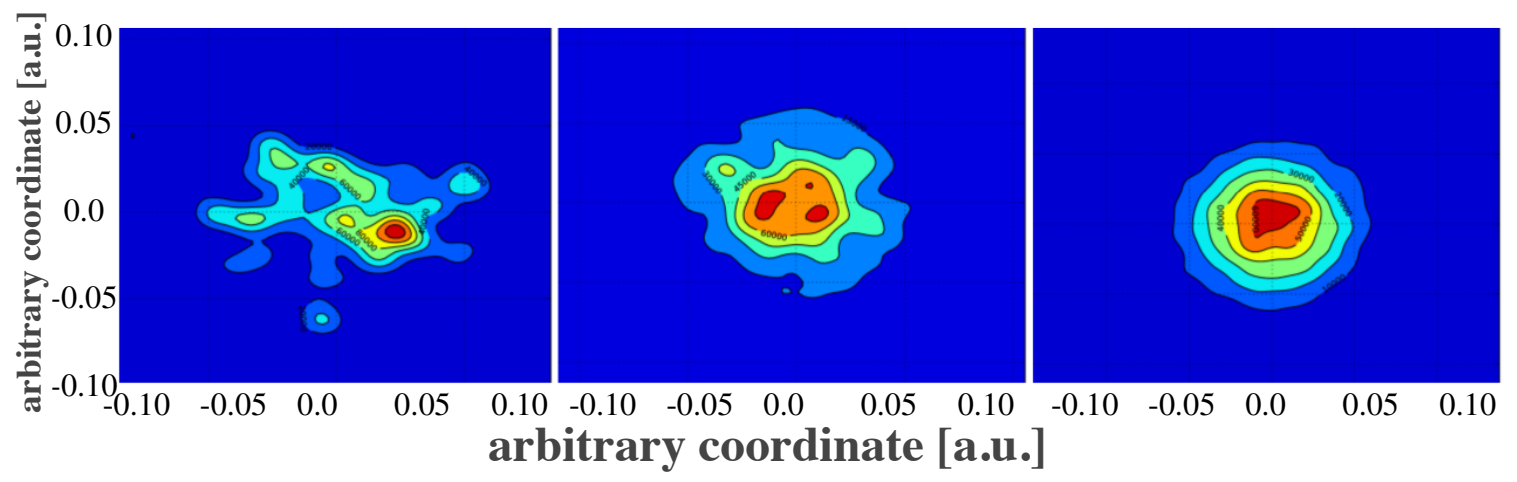

Figure 3.9. An illustration of why the bandwidth parameter, $h$ should depend on sample size. $h$ is fixed across the three contours. The contours represent the distribution of the simulated muon beam at the entrance of the MICE upstream tracker. The sample sizes change from $1000 \rightarrow 10,000 \rightarrow 100,000$ going from left to right. $h$ is too small for the smallest sample size of 1000 (left) and optimal for the largest sample size of 100,000 (right).

ure 3.9 is an illustration of this; the contour plots of two arbitrary coordinates and the corresponding phase-space densities are estimated using the KDE technique.

\subsection{Summary}

The kernel density estimation (KDE) technique has been introduced: similar to the histogram, it is a non-parametric density estimation technique; it estimates the density without an assumption about the functional form of the data. Unlike the histogram, it uses weight functions that are centered at each data point. As a result, each data point directly contributes a weighted density value towards the estimated distribution. Similar to the histogram, the width of the weight (kernel) function can be tuned to represent the dataset under study better. The parameter used in tuning the kernel width is known as the "bandwidth" parameter; it is selected such that the deviation of the estimated density from the true density, known as the mean integrated square error (MISE) is minimized. The true density in the case of MICE muon beam (Fig. 3.3) is close to a Gaussian; hence the true density is taken to be the normal distribution. This bandwidth selection approach is known as the normal 
reference rule or Scott's rule of thumb. In Chapter 4, the KDE is applied to various simulated beam distributions, including the MICE muon beam. 


\section{CHAPTER 4 \\ NOVEL APPLICATION OF KDE IN SIMULATION}

\subsection{Motivation}

The application of the kernel density estimation (KDE) technique to a variety of simulated lattices, including MICE lattices with flat $\mathrm{LiH}$ and wedge-shaped polyethylene absorbers, is studied in this chapter. Transverse beam cooling as well as direct and reverse emittance exchange are demonstrated. A large dispersion can be introduced in the MICE muon beam in simulation; this type of simulated data can be used to demonstrate direct emittance exchange (transverse heating for longitudinal cooling). The MICE experimental data, however, have a relatively small natural dispersion; as a result, when input into G4beamline for tracking of muons across the wedge, they can demonstrate only reverse emittance exchange (transverse cooling combined with longitudinal heating).

Sections 4.2 through 4.5 cover the results of simulations carried out using the G4beamline [49] software package. In addition to G4beamline, for generating the initial beam distribution, Monte Carlo simulation routines in the MICE Analysis User

Software (MAUS) [43] and Xboa [50] have been used. This initial beam is Gaussian in shape. For the case of the flat $\mathrm{LiH}$ absorber, the input beam starts at the center of the center coil in the upstream tracker and is matched to the field of the upstream solenoid. The first simulation study was on a beam of muons traversing a quadrupole magnet where no change in the muon beam density was observed. 
Table 4.1. Simulation parameters for studying a beam of muons of input emittance of $3 \pi$ $\mathrm{mm} \cdot \mathrm{rad}$ (to keep the notation short, the units of emittance will be written as $\mathrm{mm}$ in what follows) and reference momentum of $200 \mathrm{MeV} / c$, traversing a quadrupole magnet.

\begin{tabular}{lc}
\hline \hline Parameter & Value \\
\hline Quad Dimensions (Iron Length $\times$ Radius) & $0.46 \times 0.38 \mathrm{~m}$ \\
Quad Field Gradient & $1.15 \mathrm{~T} / \mathrm{m}$ \\
Field Region (Field Length $\times$ Aperture) & $0.396 \times 0.301 \mathrm{~m}$ \\
Beam Type & Gaussian \\
$\sigma_{x}$ and $\sigma_{y}$ & $0.03 \mathrm{~m}$ \\
$\sigma_{p_{x}}$ and $\sigma_{p_{y}}$ & $0.003 \mathrm{GeV} / c$ \\
\hline
\end{tabular}

\subsection{Test with a Quadrupole Magnet}

The KDE routine has been applied to a simulated muon beam traversing a quadrupole magnet. The purpose of the study was to confirm that the estimated muon beam density using the KDE technique is conserved along a quadrupole magnet (quad magnets are one of the most common lattice elements in an accelerator). A generic quadrupole magnet geometry was constructed using G4beamline with the specifications shown in Table 4.1. The generated input beam was selected to be Gaussian in shape using G4beamline (Table 4.1). The beam sample consisted of 100,000 muons with a reference momentum of $0.2 \mathrm{GeV} / c(200 \mathrm{MeV} / c)$. Two G4beamline virtual detectors are placed in the quadrupole magnet, one at the upstream and the other at the downstream quad field boundaries. The phase-space coordinate of each muon is extracted from the virtual detectors in G4beamline. The transverse coordinates in a quadrupole magnet are not correlated. As a result, the two-dimensional (2D) $\left(x, p_{x}\right)$ and $\left(y, p_{y}\right)$ phase-space planes are separately analyzed (decoupled) using the 

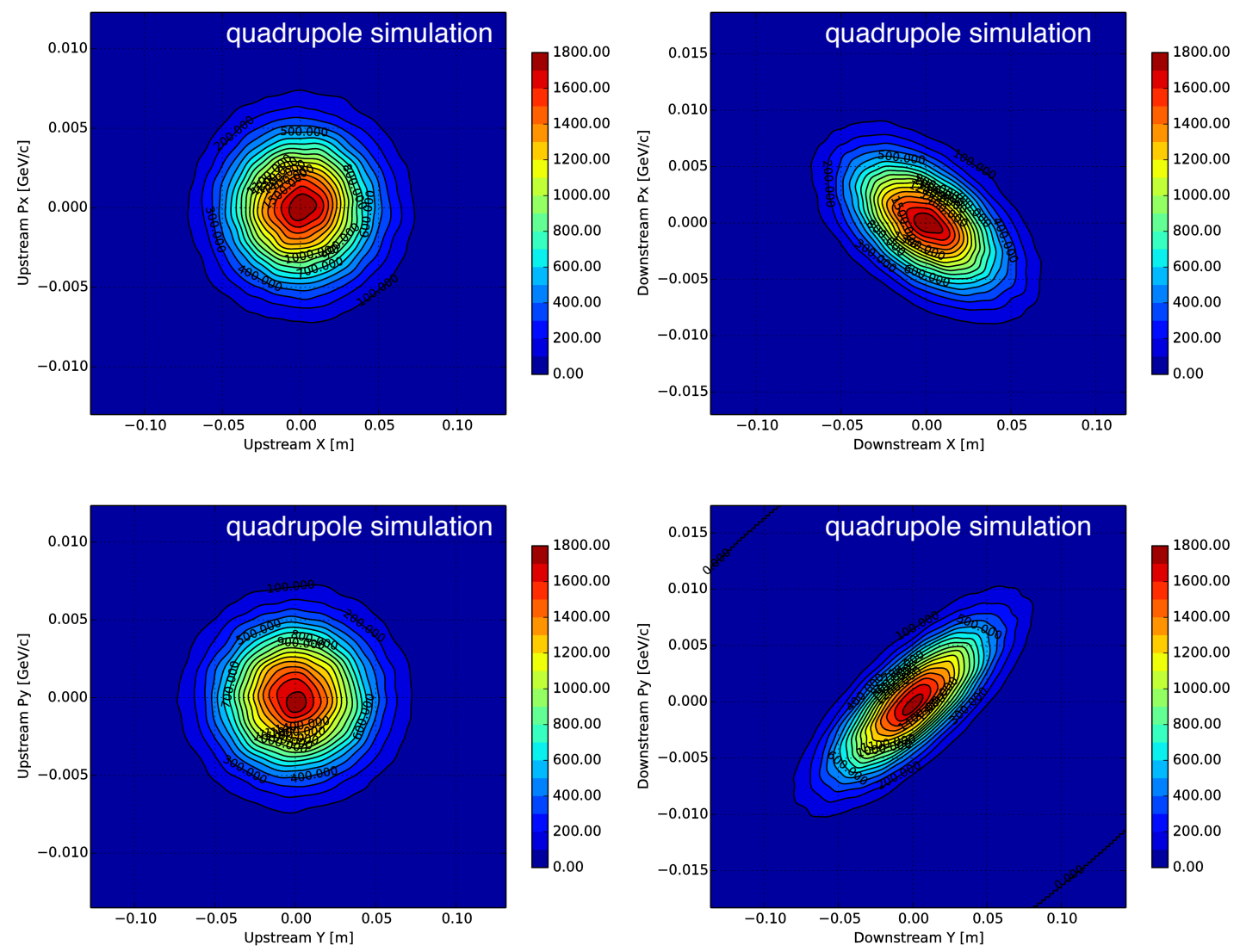

Figure 4.1. $\left(x, p_{x}\right)$ (top) and $\left(y, p_{y}\right)$ (bottom) phase-space contours (drawn by contour lines) for a muon beam distribution going from an upstream (left) to a downstream point (right) inside a quad magnet. The quadrupole is focusing along $x$ and de-focusing along $y$, hence the asymmetry between $x$ and $y$ planes.

KDE technique. This simulation study was the first application of the KDE technique to a focusing channel before its application to a MICE lattice (where the transverse coordinates are coupled due to the presence of solenoid fields). Contour plots of each of the $\left(x, p_{x}\right)$ and $\left(y, p_{y}\right)$ distributions are shown in Figure 4.1; the distributions are colored based on the KDE density values. The phase-space coordinate of each muon is extracted from the virtual detectors in G4beamline. The contours have constant density values and particles residing within each contour have estimated densities greater than or equal to the contour's estimated density [48]. The plots illustrate that the beam density does not change when the beam travels from the entrance of the quadrupole magnet to its downstream field boundary; this is in agreement with 
Liouville's theorem, which states that in the absence of energy loss, the phase-space distribution function is constant. To produce the plots, the 2D coordinates are then stacked into a single 2D array, and grid points (used in forming the contour plots) are constructed for each transverse coordinate. The grid points are bounded by the minimum and maximum $x, p_{x}, y, p_{y}$ values, with 1,000 points in-between, in each direction. To estimate the density, Gaussian kernel functions with fixed kernel widths (determined using Scott's normal reference rule [45]) are centered at each grid point. As shown in Figure 4.1, closer to the beam center (beam core), the density values are higher than in the beam periphery. This general feature can be used to extract a quantitative measure of the change in density for a given phase-space volume: the constant density values corresponding to each contour can be plotted as a function of the area (2D phase-space volume) enclosed by the contour (Fig. 4.2). The first attempt at calculating the volume (or the $x, p_{x}$ and $y, p_{y}$ area) is made using Green's theorem [51]; one can take a contour $C$ and bound an area, $A$ with it. If one then takes $X$ to be a function of $x$ and $Y$ a function of $p_{x}$, the area $A$ is:

$$
\oint_{C}\left(X d x+Y d p_{x}\right)=\iint_{A}\left(\frac{\partial X}{\partial x}-\frac{\partial Y}{\partial p_{x}}\right) d x d p_{x}
$$

where $\frac{\partial X}{\partial x}$ denotes the partial derivative of $X, \oint_{C}$ the integral over a closed loop for the contour $C$, and $\iint_{A}$ the $2 \mathrm{D}$ integral over $x$ and $p_{x}$. The same relation holds for $\left(y, p_{y}\right)$.

Figure 4.2 is the plot of the estimated contour density using the KDE routine versus the $2 \mathrm{D}$ area calculated using the Green's theorem. As mentioned earlier, in a quadrupole magnet, the 2D coordinates in each transverse plane are not correlated. In the case of solenoidal fields, there is a correlation among all transverse coordinates $x, p_{x}, y$, and $p_{y}$ and because Green's theorem does not take these correlations into account (Eq. 4.1 lacks a covariance matrix term), it will not be used in calculating the volume of the MICE muon beam. Instead, the volume is calculated using the Monte 

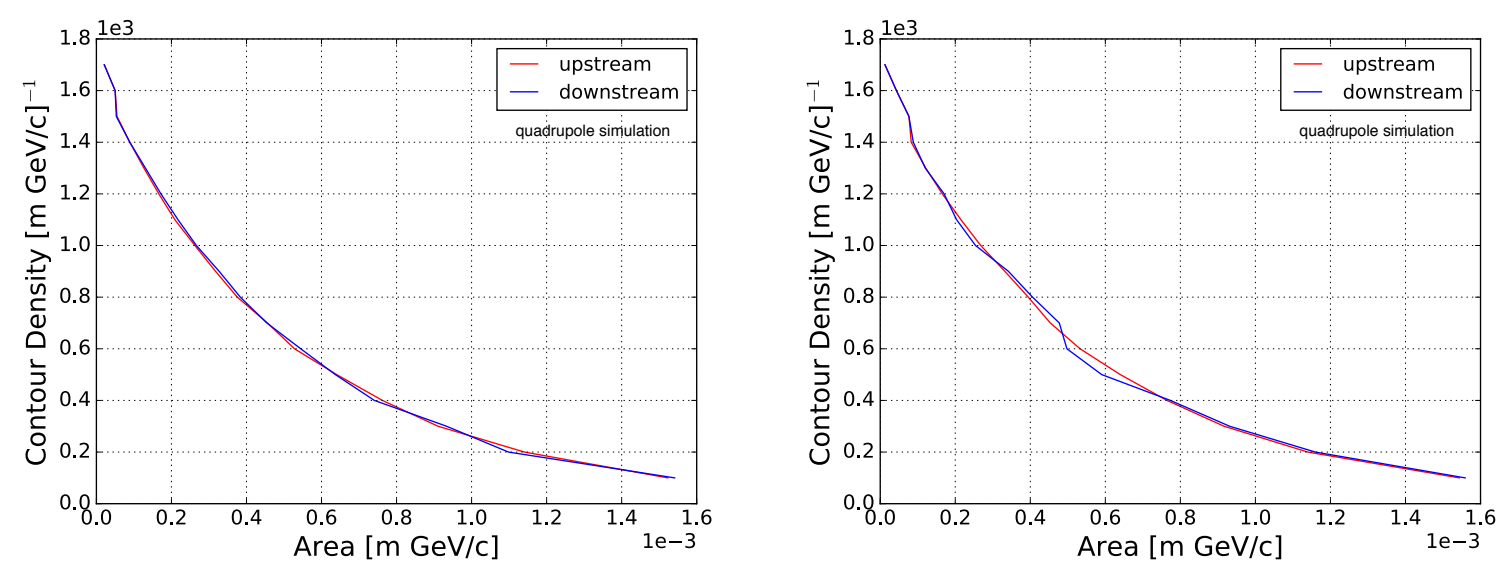

Figure 4.2. The contour density vs. beam area, from an upstream point in the quadrupole magnet to a downstream point, for the $2 \mathrm{D}$ (left) $\left(x, p_{x}\right)$ and (right) $\left(y, p_{y}\right)$ beam distributions. The slight discrepancy between upstream and downstream curves in the $\left(y, p_{y}\right)$ distribution is an artifact of the limited number of density contours considered.

Carlo approach (more on that below). The analysis in this section has demonstrated that in a focusing channel (such as a quadrupole magnet) when the effect of energy loss in the absorber is absent, the KDE density is conserved. Section 4.3 covers the analysis of a muon beam that traverses the MICE experimental setup, containing a solenoidal focusing channel (instead of a quadrupole focusing element).

\subsection{Simulated MICE Baseline Lattice}

The first simulation that tested the performance of the KDE technique in a lattice similar to MICE is a so-called MICE "baseline" lattice. The baseline lattice (Fig. 2.11) is a MICE lattice in which all the spectrometer solenoid coils are operational and running at design currents. The baseline lattice is in contrast with the MICE experimental lattice where one of the downstream matching coils is off due to an incident in 2015 (which rendered it nonoperational). Two different absorber configurations are studied: a $65 \mathrm{~mm} \mathrm{LiH}$ absorber and no absorber (empty-absorber setting with absorber holder). The two lattice configurations (with or without absorber) have the same optics and magnet configurations. The on-axis magnetic field, average momentum, and emittance across the $65 \mathrm{~mm} \mathrm{LiH}$ absorber (displayed as a 

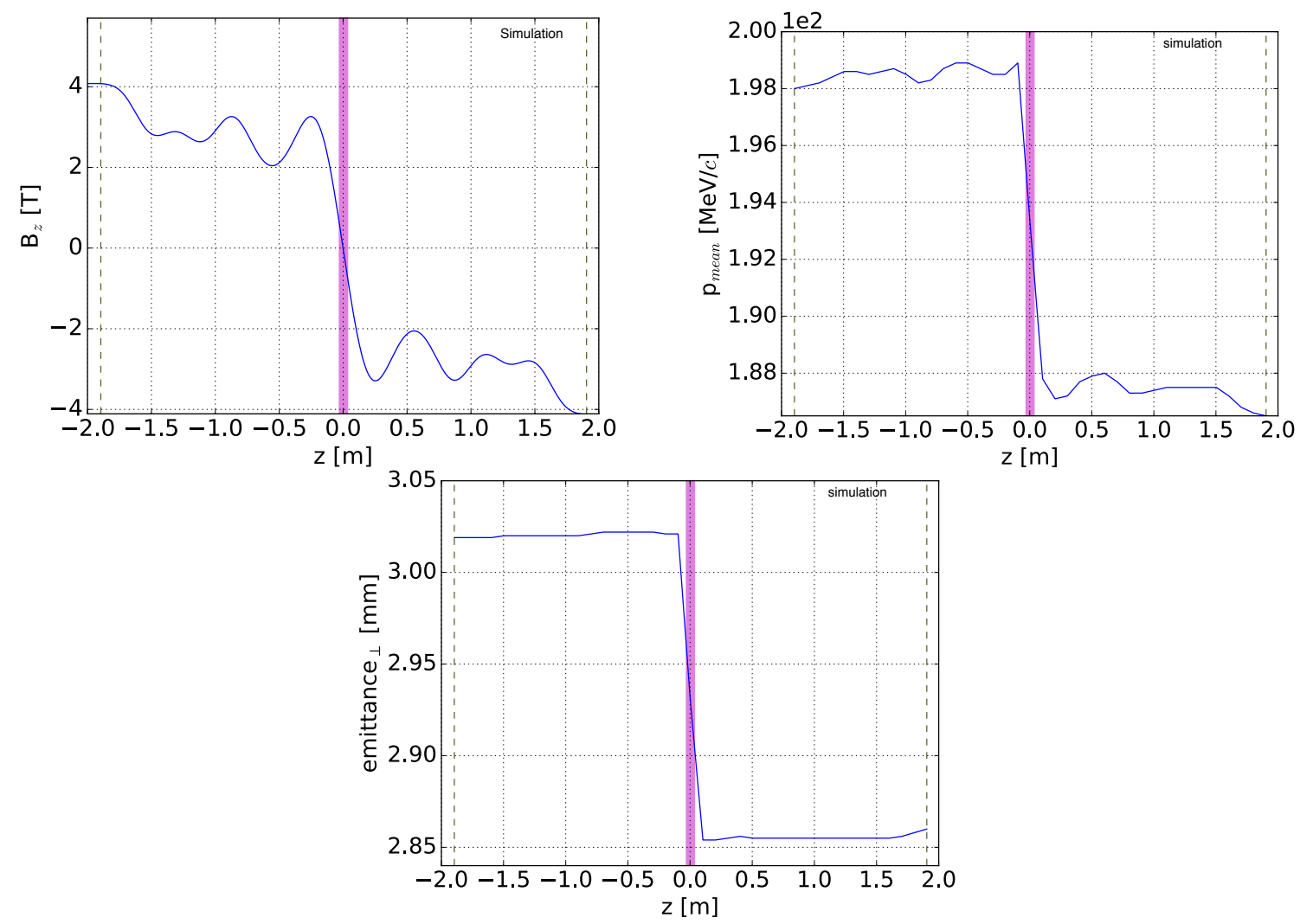

Figure 4.3. Plots of the on-axis magnetic field (upper left), average momentum (upper right), and emittance (lower middle) for the MICE baseline lattice study. Note the decrease across the absorber for all three plots. The B-field at the exit of the upstream tracker is at $4 \mathrm{~T}$ and flips sign at the absorber to minimize the build-up of the net angular momentum. 
Table 4.2. Currents of upstream SS and AFC as specified in G4beamline MICE lattice. The downstream spectrometer coils have the same currents with flipped signs.

\begin{tabular}{lc}
\hline \hline Coil & Current $\mathbf{A} / \mathbf{m m}^{2}$ \\
\hline End1 & 94.6 \\
Center & 106.7 \\
End2 & 89.1 \\
Match2 & 95.9 \\
Match1 & 82.9 \\
Upstream Focus & 79.7 \\
\hline
\end{tabular}

colored stripe at $z=0$ ) are shown in Figure 4.3. The currents in the spectrometer solenoid (SS) modules (shown in Table 4.2) are configured such that a constant $\pm 4 \mathrm{~T}$ field is maintained within the tracker volumes. In addition, the currents in both the SS and AFC modules are such that their signs flip at the center of the absorber to prevent net angular momentum build-up. The input beam emittance and reference momentum are set to $3 \mathrm{~mm}$ and $140 \mathrm{MeV} / c$, respectively [48]. The choice of small input emittance is to ensure minimized beam loss. The beam file containing the information on the initial beam distribution is generated using the Xboa [50] routine. The G4beamline [49] simulation is then run using the initial beam distribution for simulation of 10, 000 muons from the center of the center coil in the upstream tracker to the center of the center coil in the downstream tracker. The optics and the magnet currents are optimized to ensure that the beam is matched downstream of the absorber, leading to the $100 \%$ transmission of all muons to the downstream tracker reference plane. The $\left(x, p_{\mathrm{x}}\right)$ muon beam distributions, upstream (leftmost plots) and downstream (rightmost plots) of the $65 \mathrm{~mm}$ LiH absorber (lower plots) are shown in 

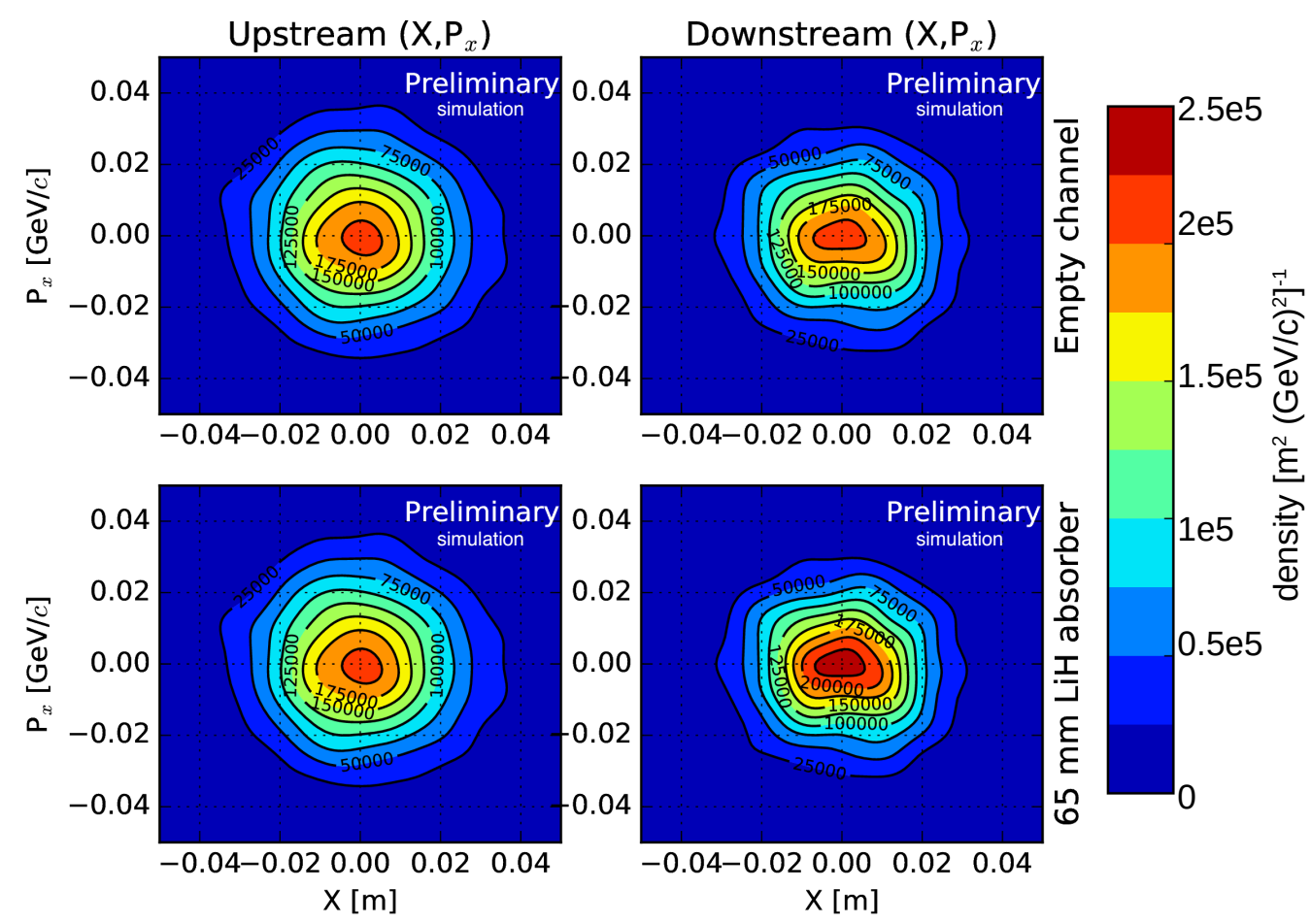

Figure 4.4. Phase-space contours before and after the LiH absorber [48].

Figure 4.4 ; the $2 \mathrm{D}$ contours are the $\left(x, p_{\mathrm{x}}\right)$ slices of the $4 \mathrm{D}$ transverse phase space. The upstream and downstream curves on these plots are the beam distributions at the tracker reference planes (upstream and downstream tracker stations closest to the absorber). The upper plots in Figure 4.4 illustrate that there is no change in density (per Liouville's theorem) for a muon beam that does not traverse an absorber (empty channel case). For the channel with a $65 \mathrm{~mm} \mathrm{LiH} \mathrm{absorber} \mathrm{(the} \mathrm{lower} \mathrm{pair}$ of plots), the cooling is qualitatively demonstrated as an increase in the core density from the upstream to the downstream tracker reference plane. The core of the beam is the region that contains the highest density value compared with the rest of the distribution. As the beam traverses the absorber, muons in the beam periphery migrate towards the beam core as a result of beam cooling. The scattering effect (heating effect explained in Section 2.1) can simultaneously cause a small subsample of muons to migrate from the beam core to periphery (heating effect); however, the 
absorber, the optics, and solenoidal focusing fields are chosen such that the cooling effect is dominant. Hence, most muons migrate from the beam periphery to the core (cooling). In this thesis, one standard deviation from the center of the MICE muon beam distribution is selected as the core; for a Gaussian beam in the four-dimensional phase space, one standard deviation from the beam center contains $9 \%$ of the total muon sample.

Figure 4.5 illustrates a more quantitative approach to characterizing the change in density compared with the contour plots. ${ }^{26}$ The fourth root of the volume is the mean radius of the $4 \mathrm{D}$ hyper-ellipsoid, with zero representing the beam center. The log scale plots are used to clarify that the area under the curves is conserved (both upstream and downstream distributions integrate to the same count of muons). In the baseline lattice, the areas under the curves are expected to be conserved because there is $100 \%$ transmission of all muons across the focus coils, in the two absorber configurations studied. Similar to Figure 4.4, with a $65 \mathrm{~mm} \mathrm{LiH}$ absorber in the channel, the density increases at the core of the beam and decreases at larger mean beam radii (the beam periphery). As expected from Liouville's theorem, in an empty lattice (no absorber in place, but with focus-coils present), no change in density is observed as the beam travels from the upstream to the downstream tracker reference plane.

In addition to the contour distributions and the density versus mean radius plots, the evolution of the phase-space density across the absorber can be studied (following the same format as the emittance evolution plot in Fig. 4.3). The evolution of the core density is shown in Figure 4.6; it illustrates the evolution of the $9^{\text {th }}$ percentile contour from the upstream to the downstream tracker reference plane and

${ }^{26}$ In the leftmost plots of Figure 4.5 the core of the distribution is better visualized if the fourth root of the volume is taken; volume values become smaller by taking the fourth root. 


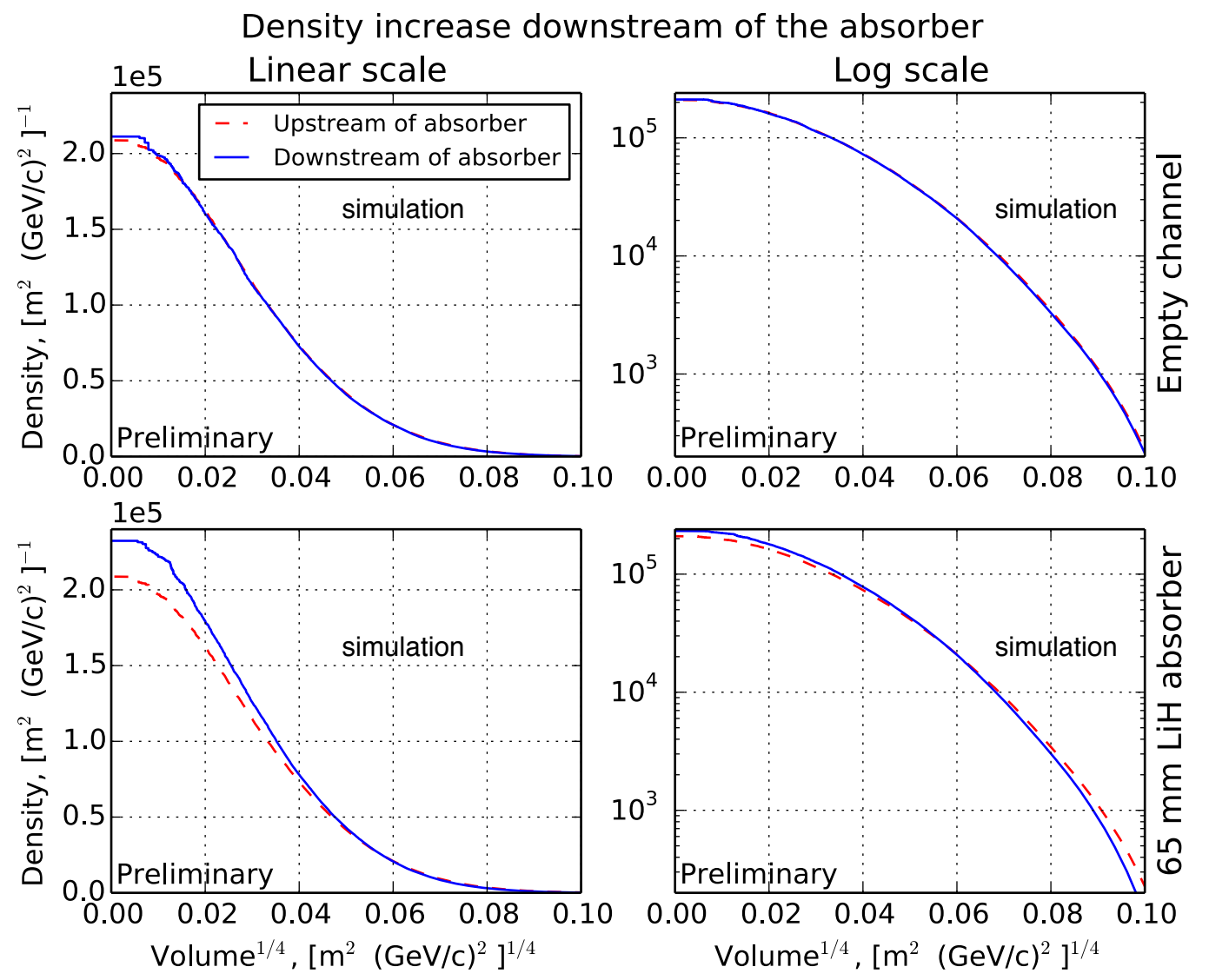

Figure 4.5. Density versus the average beam radius in the $4 \mathrm{D}$ phase-space. The density corresponds to the phase-space density of a contour of constant density while the volume is the volume enclosed inside this contour. The contour corresponding to the core of the beam has a mean radius value of $0.02\left[\mathrm{~m}^{1 / 2}(\mathrm{GeV} / c)^{1 / 2}\right][48]$. 


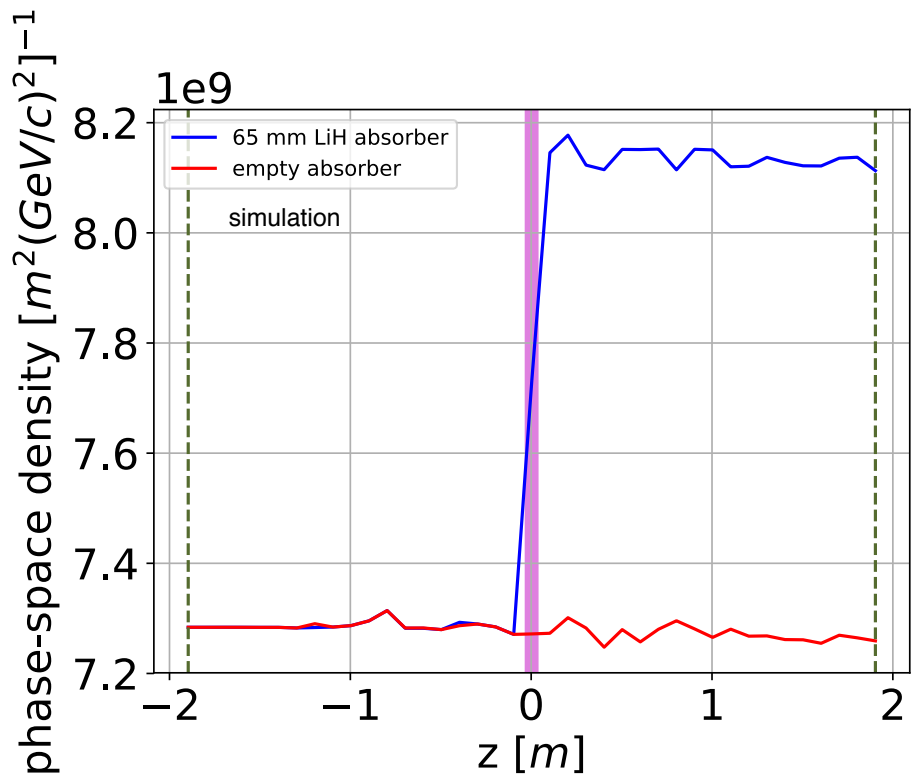

Figure 4.6. Evolution of the density of the $9^{\text {th }}$-percentile contour for two absorber configurations: $65 \mathrm{~mm} \mathrm{LiH}$ absorber and no absorber. The increase in density from the upstream $(z \approx-1.8 \mathrm{~m})$ to the downstream $(z \approx 1.8 \mathrm{~m})$ tracker reference plane (vertical dashed green lines) is a result of beam cooling.

demonstrates an increase in the core density (similar to Fig. 4.5), where the core density increases as a result of muons migrating from the beam periphery to the beam core. The beam core has been selected to be the one standard deviation from the beam center. For a four-dimensional Gaussian beam, the one standard deviation from the center of the distribution is the $9^{\text {th }}$-percentile contour [26]. The MICE beam is nearly Gaussian, hence its one standard deviation is also taken as the the contour containing $9 \%$ of the total muon sample. The evolution plot in Figure 4.6 demonstrates an increase in the core density of about $12 \%$.

To produce the core density evolution plot, the kernel density estimator is used to (the process of summing the kernel functions centered at each data point) re-estimate the density over the core muons, once a core contour is found. The idea is to first estimate the density everywhere (not just at the core of the beam) by summing over kernel functions of fixed widths centered at each muon. The widths of the kernel functions are selected such that the resulting estimated distribution has the 


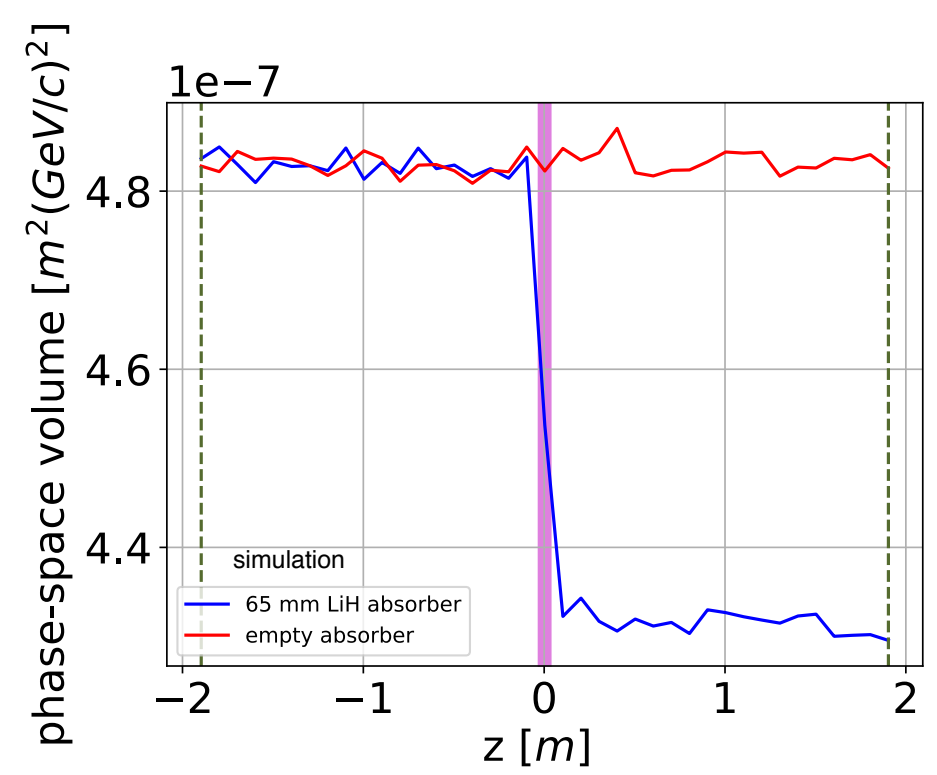

Figure 4.7. Evolution of the volume of the $9^{\text {th }}$-percentile contour for two absorber configurations of $65 \mathrm{~mm} \mathrm{LiH}$ absorber and no absorber. The reduction in volume from the upstream $(z \approx-1.8 \mathrm{~m})$ to the downstream $(z \approx 1.8 \mathrm{~m})$ tracker reference planes (vertical dashed green lines) are the results of beam cooling.

smallest deviation from the true density (true density is assumed to be Gaussian). Such kernel width, known as optimal bandwidth parameter (explained in detail in Section 3.2$),{ }^{27}$ ensures that the resulting estimated density is not overly smooth or noisy. Once the core contour is found, the transverse phase-space coordinates of core muons (muons with densities higher the density of the core contour density) are saved, and the Gaussian kernel functions are re-evaluated over them. However, this time, because the core has higher occupancy (data points are more closely spaced) than the tail, the optimal kernel width is now smaller than when the tail of the distribution was included in the density estimation process; this leads to an estimated distribution that has, on average higher density than when the density is estimated everywhere in the distribution. A comparison between the evolution plots (Figs. 4.6 and 4.7) and the density versus radius plots (Fig. 4.5) demonstrates this effect. The fourth root of

${ }^{27}$ In general, the optimal bandwidth parameter is selected such that its value is not too small for the tail (to prevent noise in the tail) and not too large for the core (to prevent over-smoothing in the core). 
the volume in Figures 4.6 and 4.7 at the upstream (the left-most green dashed vertical line) and the downstream tracker reference planes (the right-most green dashed vertical line) are $0.026[\mathrm{~m} \cdot \mathrm{GeV} / c]^{1 / 2}$ and $0.025[\mathrm{~m} \cdot \mathrm{GeV} / c]^{1 / 2}$, respectively; these values match the average beam radius value of about $0.02[\mathrm{~m} \cdot \mathrm{GeV} / \mathrm{c}]^{1 / 2}$ in Figure 4.5 for both the upstream and the downstream curves. The upstream density value in Figure 4.5 (corresponding to the mean radius value of $0.02[\mathrm{~m} \cdot \mathrm{GeV} / \mathrm{c}]^{1 / 2}$ ) is $1.6 \times 10^{5}$ $[\mathrm{m} \cdot \mathrm{GeV} / \mathrm{c}]^{-2}$, while the downstream density value (corresponding to the same mean radius) is approximately $1.8 \times 10^{5}[\mathrm{~m} \cdot \mathrm{GeV} / \mathrm{c}]^{-2}$. The percent change in the density value as the beam traverses the absorber from Figure 4.6 is therefore about 11\%, which is in agreement with the density change of about $11 \%$ shown in Figure 4.5 .

The volume can be calculated using a separate Monte Carlo (MC) approach. The MC approach can be used to calculate the volume of any contour in the 4D transverse phase space (e.g., Fig. 4.7, where phase space is divided into 1000 contours, and each contour is calculated using the MC approach). The idea is first to bound the contour under study by a hyper-rectangle. The minimum and maximum values of the muons' $\left(x, p_{x}, y, p_{y}\right)$ phase-space coordinates (in the particular contour under study) are the dimensions of the hyper-rectangle. A three-dimensional example of the bounding hyper-rectangle is shown in Figure 4.8. The next step is to throw randomly generated points (MC points) uniformly over the hyper-rectangle. The volume of the contour is then the volume of the hyper-rectangle (calculated as $\left.v_{\text {rectangle }}=\left(x_{\max }-x_{\min }\right) \times\left(p_{x \max }-p_{x \min }\right) \times\left(y_{\max }-y_{\min }\right) \times\left(p_{y \max }-p_{y \min }\right)\right)$ multiplied by the number of MC points, $n$ enclosed within the contour under study (red points in Fig. 4.8) as a fraction of the total number of generated MC points, $N$ (red and black points in Fig. 4.8): $v_{\text {rectangle }} \times \frac{n}{N}$. The MC points inside the contour are found and counted based on their density values: points inside the contour have density values greater than the contour density. In Figure 4.7, the MC approach is used 


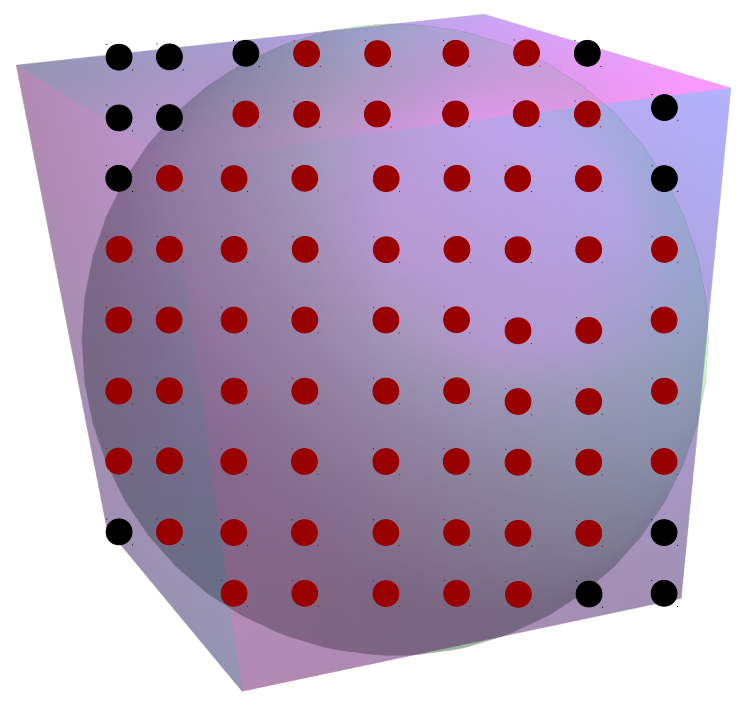

Figure 4.8. A three-dimensional illustration of the volume calculation using the MC approach. The MC points are generated uniformly within the three-dimensional bounding box. Only the MC points that reside within the bounded contour (red points) are used to calculate the contour volume.

to compute the core $\left(9^{\text {th }}\right.$-percentile) contour at $13 z$ positions, ${ }^{28}$ from the upstream to downstream tracker reference plane. The phase-space volume of the beam core is conserved in the drift regions between the upstream tracker reference plane and the absorber (from $z=-1.8 \mathrm{~m}$ to $z=-0.0325 \mathrm{~m}$ ). Once the beam core reaches the $\mathrm{LiH}$ absorber (at $z=-0.0325 \mathrm{~m}$ ) and travels through it, its phase-space volume decreases by $\approx 8 \%$, as a result of ionization cooling. There is no change in volume in the case of an empty absorber lattice (a lattice with no absorber). The core volume is conserved between the absorber and the downstream tracker reference plane (from $z=0.0325$ $\mathrm{m}$ to $z=1.8 \mathrm{~m}$ ). The fluctuations in the volume curve are due to the statistical (randomly generated points) nature of the volume calculation.

The analysis in this section has demonstrated the cooling performance of a

${ }^{28}$ Each $z$ position in the evolution plots is the location of a G4beamline virtual detector, with the most upstream virtual detector located at the upstream tracker reference plane and the most downstream one located at the downstream tracker reference plane. 
solenoidal focusing channel: in the absence of an absorber (i.e., energy loss), the estimated phase-space density and volume (in agreement with Liouville's theorem) are conserved. In the presence of a $65 \mathrm{~mm} \mathrm{LiH}$ absorber, however, there is an increase in the estimated phase-space density and a reduction in the phase-space volume at the core of the beam. This, combined with a simultaneous reduction in the density and increase in the volume of the muons residing outside of the beam core (shown in Figure 4.5), makes up the expected ionization cooling signal for a beam of muons muons at the beam periphery migrate to the beam core as a result of beam cooling.

\subsection{Simulated MICE Lattice - a "least desirable" realistic case}

The previous section demonstrated the performance of the KDE technique with a lattice configuration that had all solenoid magnet coils operating according to design. Such a lattice can be considered as the "best-case" lattice. Some of the MICE experimental data, however, was collected when one or two downstream matching coils were turned off due to an incident in 2015 (which rendered match coil 1 in the downstream SS nonoperational). Therefore, in this section, the KDE performance is evaluated for a more realistic lattice where both downstream match coils (Fig. 4.9) are turned off; this lattice is considered the least desirable realistic lattice (due to a larger mismatch in the beam exiting the absorber, compared to the majority of MICE experimental data, which was collected while only the downstream match coil 1 was

turned off). The coil currents (also used in collecting some of the MICE experimental data) are displayed in Table 4.3. The input beam distribution is generated using MAUS [43] and xboa [50] and is tracked through the G4beamline MICE lattice, traversing a $65 \mathrm{~mm} \mathrm{LiH}$ absorber. The reference momentum is $140 \mathrm{MeV} / c$, and two input emittance configurations are studied: $6 \mathrm{~mm}$ (known as the 6-140 setting) and $10 \mathrm{~mm}$ (known as the 10-140 setting). The equilibrium emittance for the two input beam configurations are the same $(5 \mathrm{~mm})$; as a result, the cooling performance is 


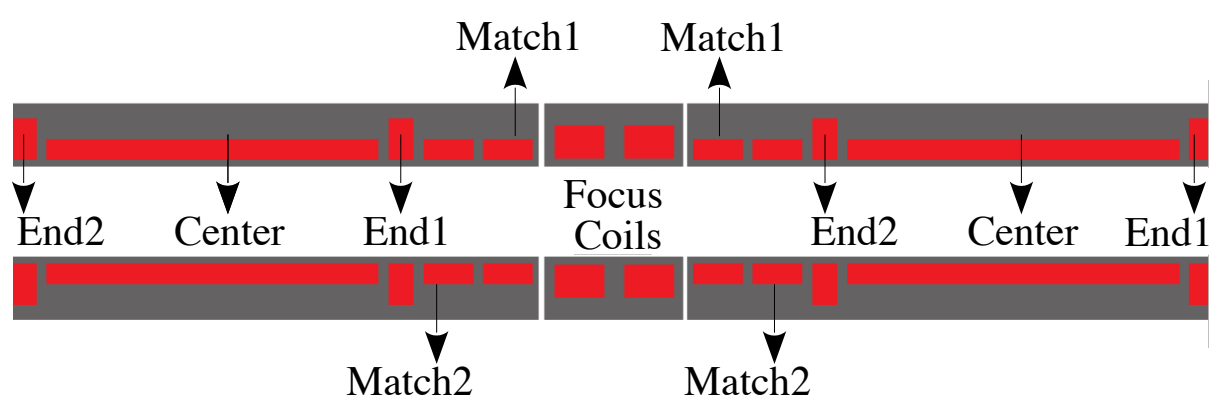

Figure 4.9. The schematics of the MICE displaying the locations of the superconducting coils in the SS and AFC.

expected to be larger for the 10-140 setting than for the 6-140 case. In each of the two settings, the input beam distribution starts with 10,000 muons. However, 89\% and $85 \%$ of muons in the respective $6-140$ and $10-140$ settings are transmitted from the upstream to the downstream tracker reference plane; the beam loss (referred to as scraping) is due to a "mismatch" (muon beam distribution not matching the radius of the tracker in size) in the beam distribution at the exit of the absorber (as a result of the turned-off downstream match coils), an effect that causes the beam to distort in shape and exceed the acceptance in the downstream tracker. The evolution of the phase-space density and volume for the 6-140 and the 10-140 input beam settings are shown in Figures 4.10a, 4.11a, 4.10b, and 4.11b. These plots are produced by summing over Gaussian kernels that are centered at each muon's 4D phase-space coordinates, $(x, p x, y, p y)$. The core muons are isolated using the estimated density information of all muons (using a binary search approach). The Gaussian kernels are then re-centered at the core muon's $4 \mathrm{D}$ phase-space coordinates; this leads to the estimated density corresponding to the core $\left(9^{\text {th }}\right.$-percentile) contour. ${ }^{29}$ The density curves in Figures 4.10a and 4.11a are therefore the densities corresponding to the core contour or the $9^{\text {th }}$ percentile contour. Likewise, the volume curves in Figures $4.10 \mathrm{~b}$ and $4.11 \mathrm{~b}$ are the $9^{\text {th }}$-percentile contour volumes. The MC routine (explained in

${ }^{29}$ The $9^{\text {th }}$-percentile contour was chosen to be the one $\sigma$ of the beam distribution following a Gaussian beam assumption. 
Table 4.3. Currents of upstream SS and AFC as specified in G4beamline MICE lattice.

The downstream spectrometer coils have the same currents with flipped signs.

\begin{tabular}{lc}
\hline \hline Coil & Current $[\mathbf{A}]$ \\
\hline Upstream End1 & 205.8 \\
Upstream Center & 205.8 \\
Upstream End2 & 205.8 \\
Uptsream Match2 & 171.9 \\
Upstream Match1 & 211.7 \\
Focus & 57.9 \\
Downstream Match1 & 0 \\
Downstream Match2 & 0 \\
Downtsream End2 & 205.8 \\
Downstream Center & 205.8 \\
\hline
\end{tabular}


Section 4.3) is used in calculating the core volume. In Figures 4.10a and 4.10b, where the beam setting under study is 6-140, the core density increases by about $16 \%$, and the core volume is reduced by about 17\%. The 10-140 plots shown in Figures 4.11a and $4.11 \mathrm{~b}$ demonstrate a larger cooling effect than the $6-140$ case: a $21 \%$ increase in the core density and $20 \%$ reduction in the core volume. This larger cooling effect is due to the larger (than the $6 \mathrm{~mm}$ input emittance) input emittance compared with the equilibrium emittance of $5 \mathrm{~mm}$. In addition, the 10-140 optics suffers from larger transmission loss than the 6-140 beam setting; the beam has a larger volume to start with, fully populating the tracker acceptance region. This causes more muons to scrape from the beam periphery downstream of the absorber, compared with the 6-140 case; for this reason, the setting used for this simulation study is known as the least desirable setting and is not further pursued.

In addition to the evolution plots, the change in all contour densities for fixed amplitude can be studied. Amplitude is the measure of the distance of each muon from the beam center in the $4 \mathrm{D}$ phase space. Its conceptual diagram in the $2 \mathrm{D}$ $\left(x, p_{x}\right)$ phase space is shown in Figure 4.12. The white curve in Figure 4.12 bounds the muon beam phase-space distribution in $\left(x, p_{x}\right)$ and represents the approximate constant amplitude of the beam 2D ellipse. One particular muon, shown with a large marker size in Figure 4.12, is illustrated as residing on a constant amplitude contour. The colors in Figure 4.12 are the density values obtained using the KDE technique. It can be observed from Figure 4.12 that several density values are associated with the same constant amplitude curve; this is because the constant-amplitude contour (white curve) tends to cross some number of constant-density contours.

To compute amplitude, first the normalized emittance, $\varepsilon_{\perp}$, is computed and then the Mahalanobis distance [53] $\left(\vec{r}^{T} \Sigma \vec{r}\right.$ of each muon in the sample from the 


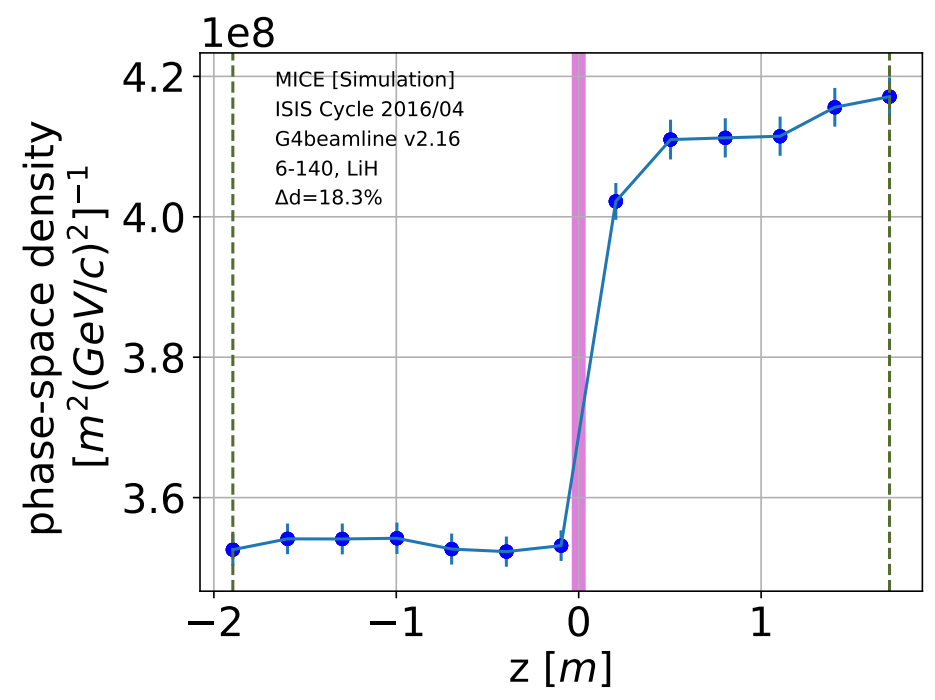

(a)

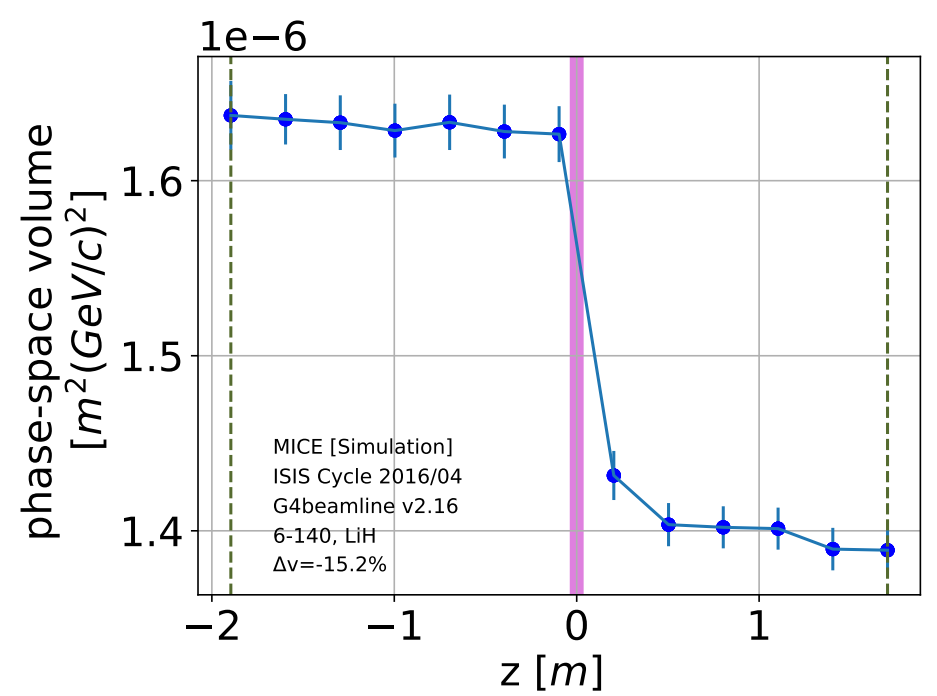

(b)

Figure 4.10. Evolution of the density (a) and volume (b) of the $9^{\text {th }}$-percentile contour for two absorber configurations of $65 \mathrm{~mm} \mathrm{LiH}$ absorber and no absorber. The changes in density and volume from the upstream $(z \approx-1.8 \mathrm{~m})$ to the downstream $(z \approx 1.8$ $\mathrm{m})$ tracker reference planes (vertical dashed green lines) are the results of beam cooling (6-140 beam setting) [52]. 


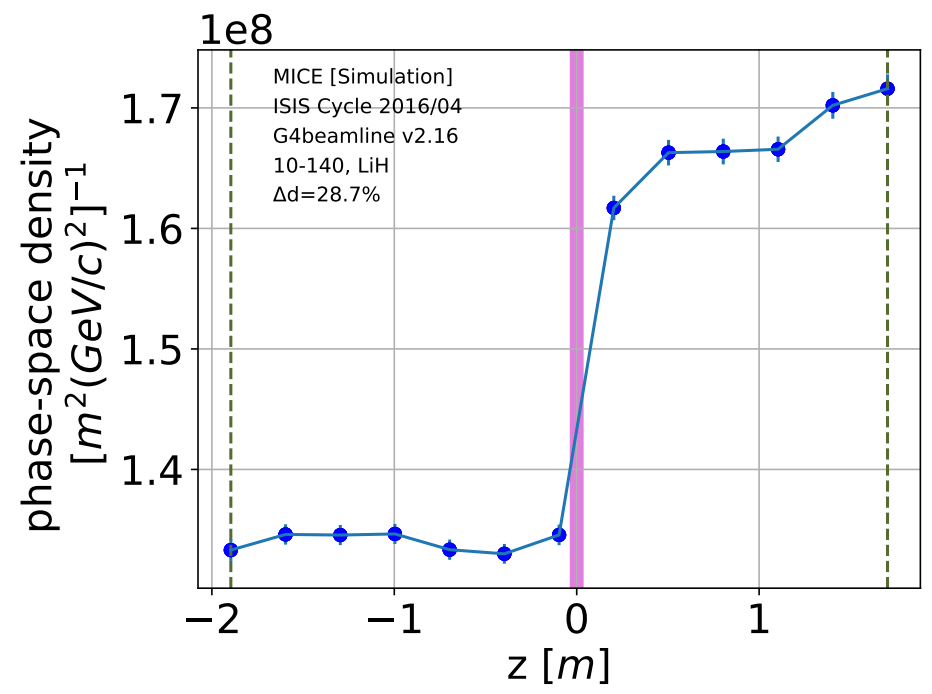

(a)

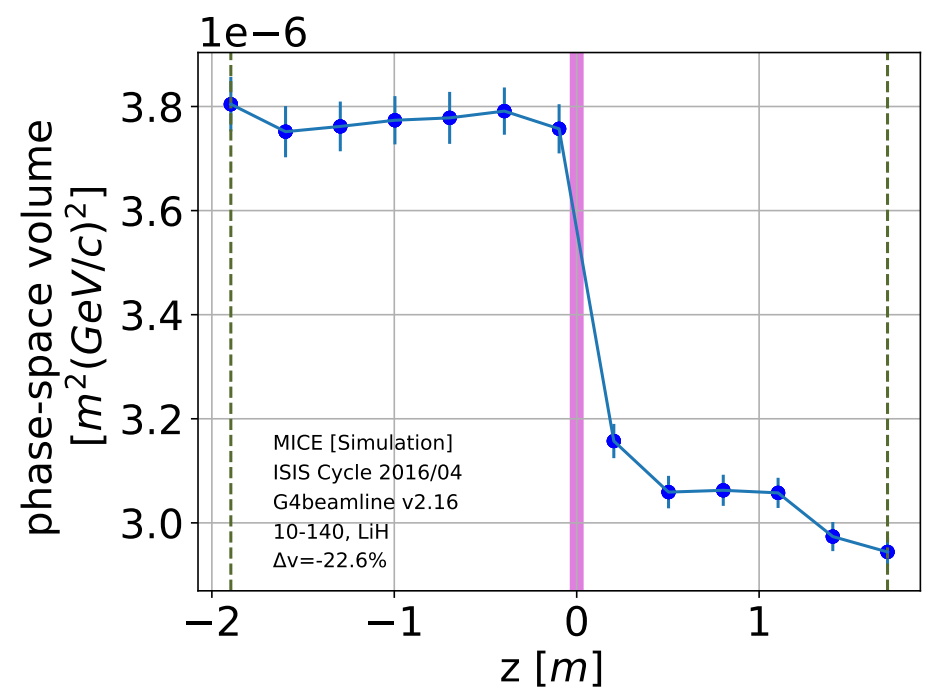

(b)

Figure 4.11. Evolution of the density (a) and volume (b) of the $9^{\text {th }}$-percentile contour for two absorber configurations of $65 \mathrm{~mm} \mathrm{LiH}$ absorber and no absorber. The changes in density and volume from the upstream $(z \approx-1.8 \mathrm{~m})$ to the downstream $(z \approx 1.8$ $\mathrm{m})$ tracker reference planes (vertical dashed green lines) are the results of beam cooling (10-140 setting) [52]. 


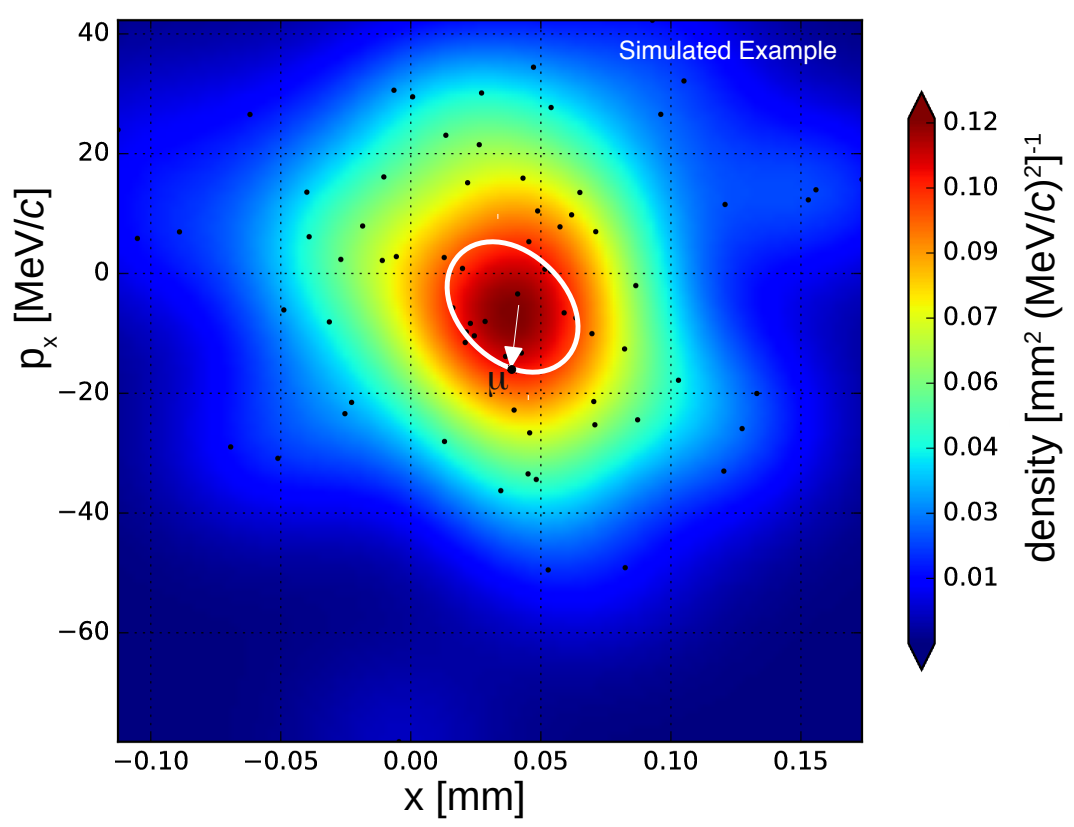

Figure 4.12. An illustration of the amplitude concept in the $x-p_{x}$ phase space. The contours of constant density are drawn using the kernel estimated density. The black dots represent the muons in the phase space, and a constant amplitude contour is shown in white.

beam center is calculated,

$$
A_{\perp}=\varepsilon_{\perp} \vec{r}^{T} \Sigma^{-1} \vec{r}
$$

where $\Sigma$ is the covariance matrix, $r$ the transverse phase space vector $\left(x, p_{x}, y, p_{y}\right)$, and $\vec{r}^{T}$ the transpose of the phase-space coordinate vector $r$. If the beam is not fully centered at the origin, $\vec{r}$ is replaced by $\vec{r}-\vec{r}_{0}$ where $\vec{r}_{0}$ represents the coordinates of the beam centroid (Fig. 4.12). Therefore, in addition to representing the emittance, amplitude accounts for the distances between muons and the beam center.

Figures 4.13 and 4.14 are plots of phase-space density estimates (obtained using the KDE technique) versus 4D transverse amplitudes for the 6-140 and 10-140 input beam settings, respectively. As shown in the topmost plots in Figures 4.13 and 4.14, the density versus amplitude curves are broad; this is because the constant amplitude surfaces extend over a number of constant density surfaces. This leads to multiple density values for the same amplitude (referring back to Fig. 4.12). The lower 

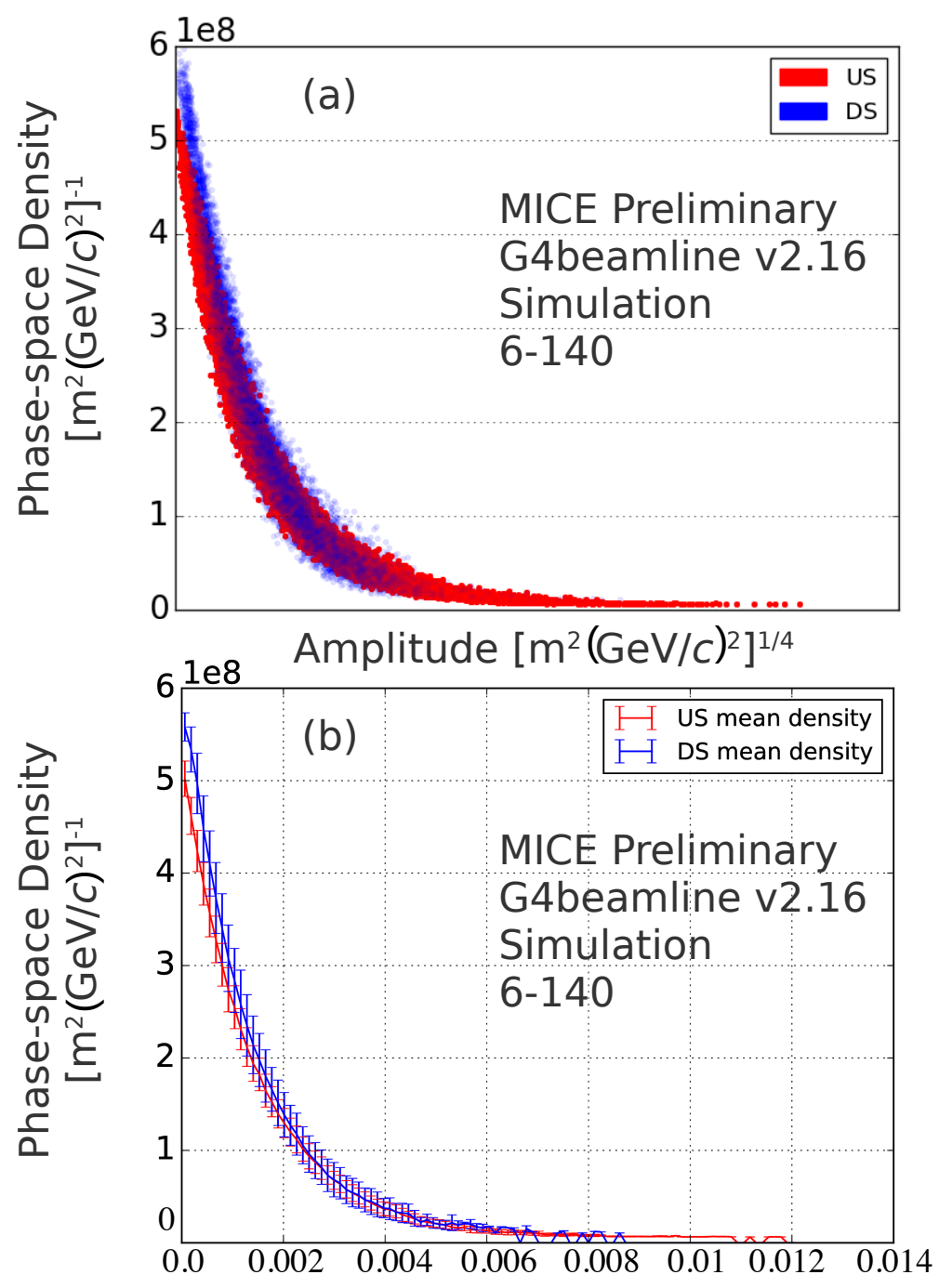

Figure 4.13. Density versus amplitude for the MICE lattice with 6-140 input beam setting [52]. 


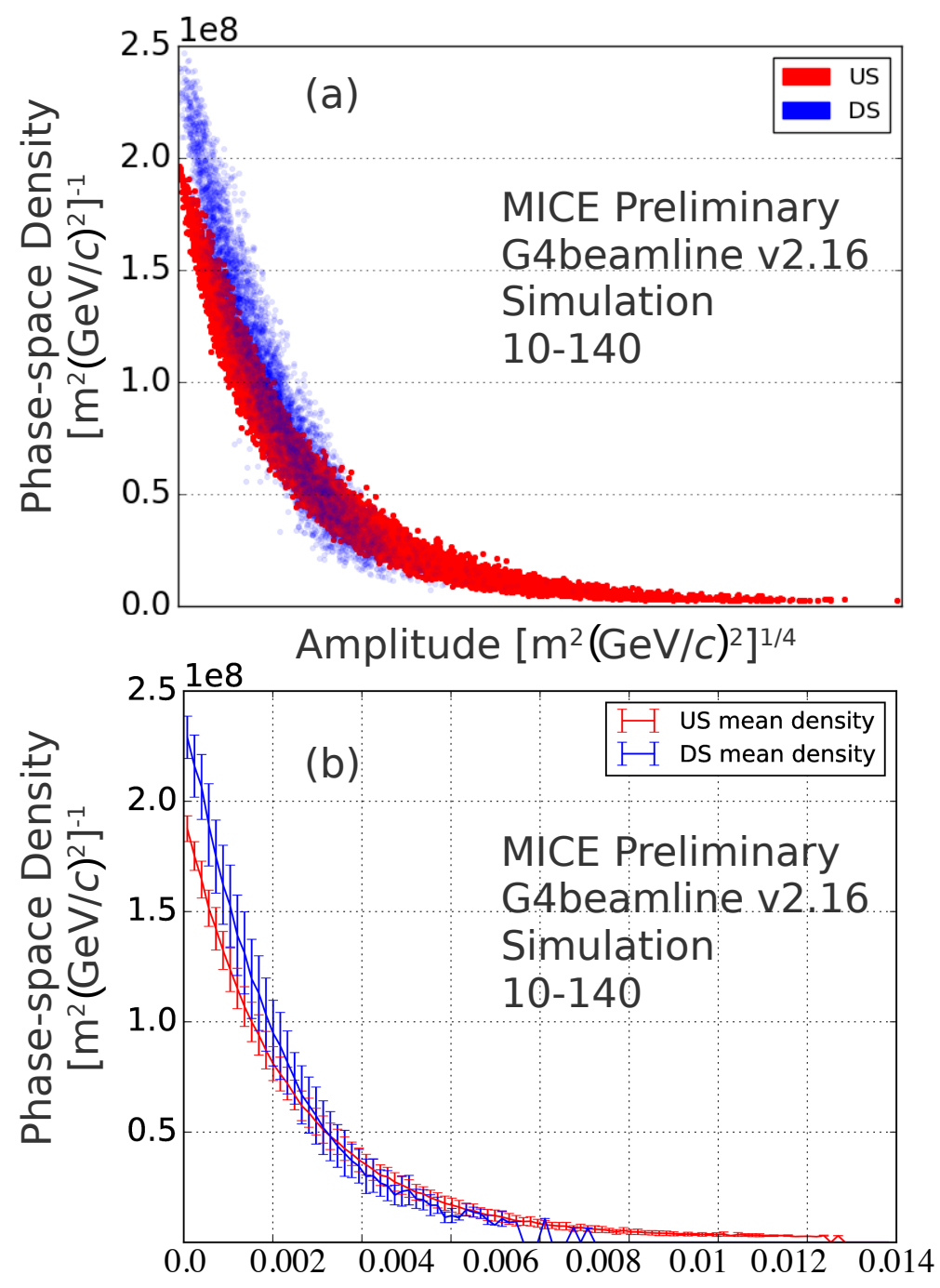

Figure 4.14. Density versus amplitude for the MICE lattice with 10-140 input beam setting [52].

plots of Figures 4.13 and 4.14 are obtained by uniformly binning the amplitudes in the upper plots and averaging over the density values residing within each bin. These average density versus amplitude plots illustrate the increase in density at smaller beam amplitudes for the 6-140 and 10-140 input beam settings. The error bars are the standard deviations of the density values within each bin. The increase in the core density (at smaller amplitudes) is observed. 


\subsection{Simulated Cooling Performance with a Wedge-shaped Absorber}

Up to this point, only simulation studies involving a flat $\mathrm{LiH}$ absorber have been covered. To demonstrate longitudinal or 6D cooling, however, a wedge-shaped absorber must be used (as described in Section 2.1). In the simulation, the wedge absorber can be used to demonstrate direct and reverse emittance exchange. In direct emittance exchange, the beam cools in the longitudinal direction cooling in exchange for transverse heating. Reverse emittance exchange leads to transverse cooling in exchange for longitudinal heating. The corresponding simulation parameters are shown in Table 4.4, and, as before, the simulated MICE lattice is constructed using G4beamline. The SS and AFC currents are the same as those used in collecting some of the MICE experimental data (shown in Table 4.5). The simulated beam must have dispersion at the location of the absorber to demonstrate (direct) emittance exchange. However, a beam distribution traversing a solenoid magnet (e.g., upstream SS module in MICE) does not preserve its dispersion. ${ }^{30}$ For this reason, instead of starting with the desired dispersion at the entrance of the upstream tracker, a dispersion is introduced in the beam at the center of the wedge (starting point is the center of the wedge; the wedge, however, has been removed to prevent any loss of dispersion in the presence of material). The absorber is then removed, and the particles are tracked in the downstream SS but using the upstream coil currents. The resulting beam is then massaged to look like an upstream beam by reversing the transverse momentum and time coordinates, $p_{x}=-p_{x}, p_{y}=-p_{y}$, and $t=-t$. At this point, the beam is input into G4beamline for tracking of 20,000 muons from the center of the upstream center coil to the center of the downstream center coil.

${ }^{30} \mathrm{~A}$ charged particle traversing a focusing solenoidal channel receives a "momentum kick" depending on where it is located in the transverse phase plane; charged particles with higher transverse momenta are kicked into lower transverse momentum values, causing the dispersion to shrink. 
Table 4.4. Parameters of the G4beamline simulation for the emittance exchange studies.

\begin{tabular}{lc}
\hline \hline Parameter & Value \\
\hline Wedge Angle $\left.{ }^{\circ}\right]$ & 45 \\
Wedge On-axis Length $[\mathrm{mm}]$ & 52 \\
$\varepsilon_{\perp}[\mathrm{mm}]$ & 6 \\
$\beta_{\perp}[\mathrm{mm}]$ & 400 \\
$D_{y}[\mathrm{~mm}]$ (dispersion) & 300 \\
$D_{x}[\mathrm{~mm}]$ & 0 \\
$p_{r e f}[\mathrm{MeV} / c]$ & 140 \\
\hline
\end{tabular}

Table 4.5. Currents of upstream and downstream SS and AFC coils as specified in G4beamline MICE lattice with a wedge absorber.

\begin{tabular}{lc}
\hline \hline Coil & Current $[\mathbf{A}]$ \\
\hline Upstream End1 & 205.7 \\
Upstream Center & 205.7 \\
Upstream End2 & 205.7 \\
Uptsream Match2 & 168.2 \\
Upstream Match1 & 191.0 \\
Upstream Focus & 129 \\
Downstream Focus & -129 \\
Downstream Match1 & 0 \\
Downstream Match2 & -195.7 \\
Downtsream End2 & -144.0 \\
Downstream Center & -144.0 \\
Downstream End1 & -144.0 \\
\hline
\end{tabular}


The coordinates in the full six-dimensional (6D) phase space are $\left(x, p_{x}, y, p_{y}\right.$, $\Delta E, \Delta t)$, where $\Delta E, \Delta t$ are the differences between each muon's energy and time and the beam distribution's average energy and time $\left(\Delta E=E_{i}-E_{\text {mean }}\right.$ and $\Delta t=t_{i}-t_{\text {mean }}$ where $i$ represents the $i^{\text {th }}$ muon in the sample). In 6D, Gaussian kernel functions are centered at each muon in the six-dimensional phase space. For tracking the evolution of the core density and volume, the next step is to isolate the core muons. In $6 \mathrm{D}$, the contour corresponding to the beam core is chosen to be the $2^{\text {nd }}$-percentile contour, containing $2 \%$ of the muon sample (the one standard deviation from the center of the beam distribution in 6D, assuming a Gaussian beam). The volume of the core contour is calculated by applying the $\mathrm{MC}$ volume calculation routine to the core muons. The evolutions of the phase-space density and volume are shown in Figures 4.15a and $4.15 \mathrm{~b}$. The error bars are the standard deviations of the densities and volumes of the 10 subsamples of 1,000 muons. The 6D phase-space density and volume are shown in Figure 4.15a; the large error bars are due to small statistics within the $2^{\text {nd }}$ percentile contour. The longitudinal density and volume evolution plots (Figs 4.16a and 4.16b) illustrate the evolution of the core contour in the 2D longitudinal phase space; the core contour in the $2 \mathrm{D}$ corresponds to the $24^{\text {th }}$-percentile contour, containing the $24 \%$ subsample of all muons in the sample. The longitudinal cooling shown in Figures $4.16 \mathrm{a}$ and $4.16 \mathrm{~b}$ combined with the transverse heating shown in Figures 4.17a and 4.17b illustrate an unambiguous sign of emittance exchange (longitudinal cooling in exchange for transverse heating). If no dispersion is introduced via the method described above, by the time the MICE muon beam arrives at the wedge, it has a small natural dispersion $\left(D_{\mathrm{y}} \approx 50 \mathrm{~mm}\right)$. This natural dispersion, if smaller than $300 \mathrm{~mm}$, is not enough to demonstrate (direct) emittance exchange; however, it can be used to demonstrate reverse emittance exchange: the beam acquires a momentum spread after passing through the wedge leading to longitudinal heating and transverse cooling. To demonstrate reverse emittance exchange, the same G4beamline lattice as 


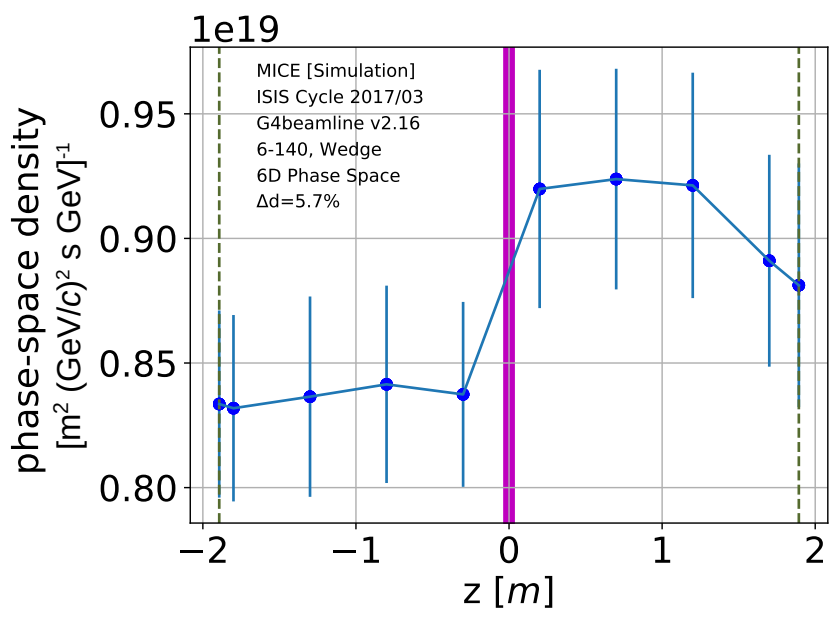

(a)

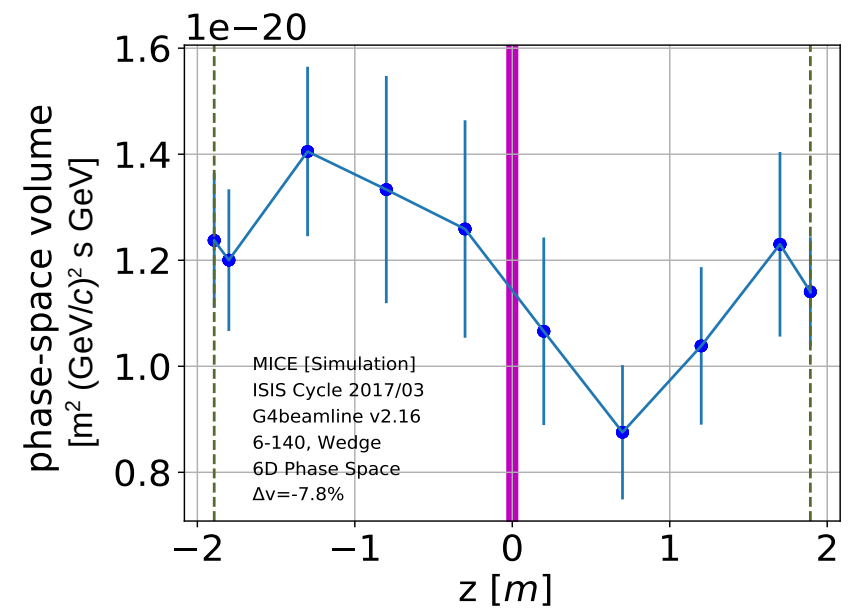

(b)

Figure 4.15. Evolution of the density (a) and volume (b) of the $2^{\text {nd }}$-percentile contour for the wedge absorber simulation. The $6 \mathrm{D}$ density and volume changes from the upstream $(z \approx-1.8 \mathrm{~m})$ to the downstream $(z \approx 1.8 \mathrm{~m})$ tracker reference planes (vertical dashed green lines). 


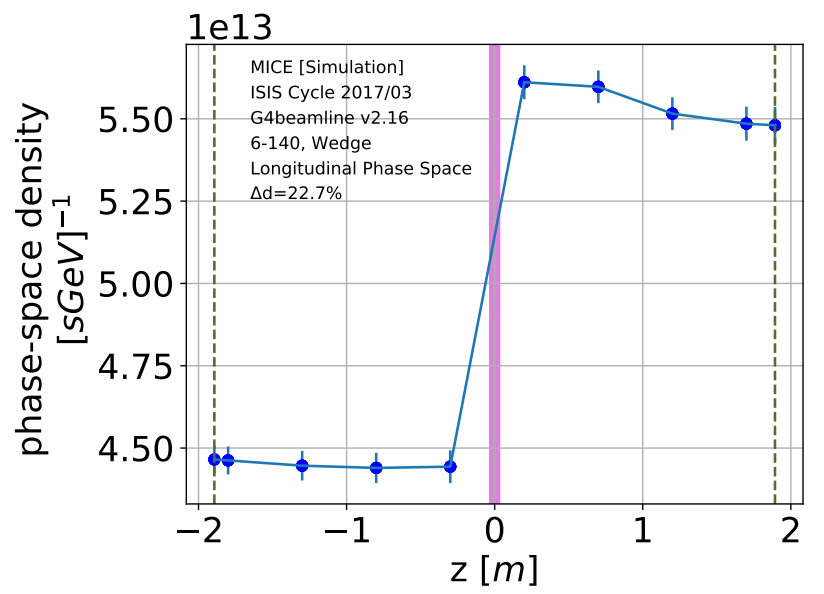

(a)

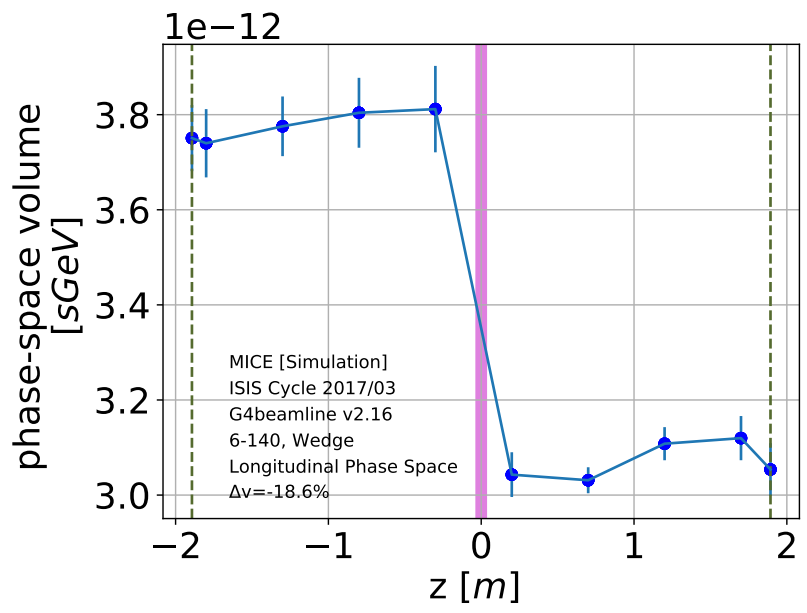

(b)

Figure 4.16. Evolution of the density (a) and volume (b) of the $24^{\text {th }}$-percentile contour for the wedge absorber simulation. The changes in density and volume from the upstream $(z \approx-1.8 \mathrm{~m})$ to the downstream $(z \approx 1.8 \mathrm{~m})$ tracker reference planes (vertical dashed green lines) are the results of longitudinal beam cooling. 


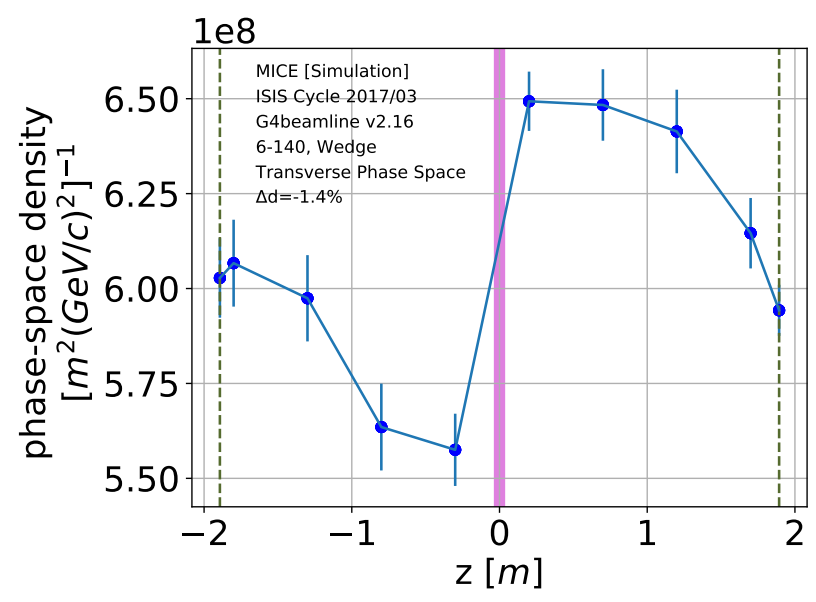

(a)

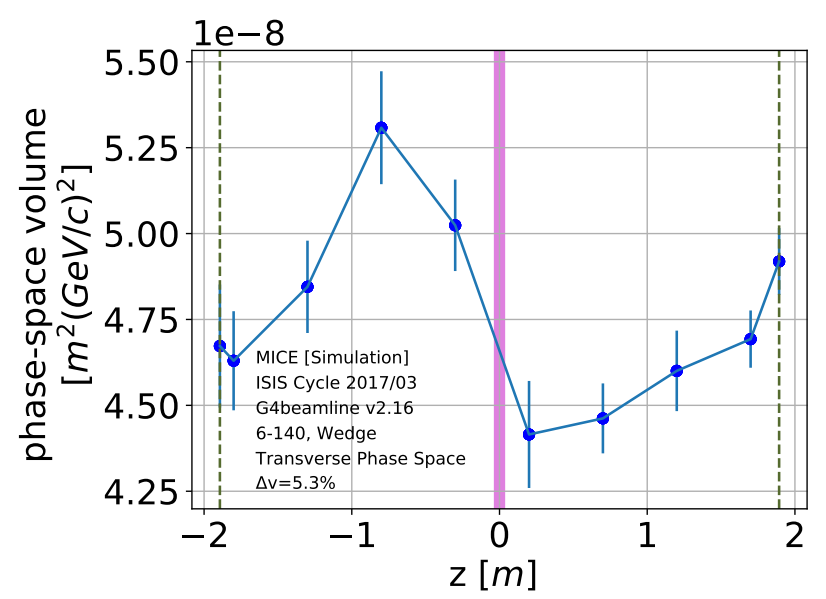

(b)

Figure 4.17. Evolution of the 4D density (a) and volume (b) of the $9^{\text {th }}$-percentile contour for the wedge absorber simulation. The changes in transverse density and volume from the upstream $(z \approx-1.8 \mathrm{~m})$ to the downstream $(z \approx 1.8 \mathrm{~m})$ tracker reference planes (vertical dashed green lines) are the results of transverse beam heating. 
for the emittance exchange analysis was used. The input beam, however, is the MICE experimental data collected in December 2017 (reconstructed position and momentum of each muon at the most upstream tracker plane). A sample of 100, 000 muons from experimental data was input to G4beamline for simulating the distribution of muons at the five tracker stations in each tracker, moving from the most upstream to the most downstream tracker station while traversing the wedge absorber (a so-called "hybrid MC approach"). Figures 4.18a through 4.20b demonstrate 6D beam heating and the reverse emittance exchange effect: transverse cooling (density increase of 35\% and volume reduction of 25\%) and longitudinal heating (density reduction of $56 \%$ and volume increase of $55 \%$ ). 


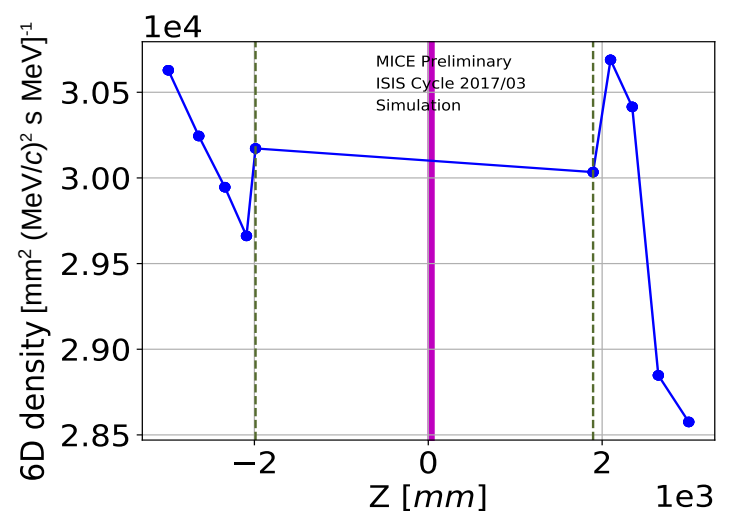

(a)

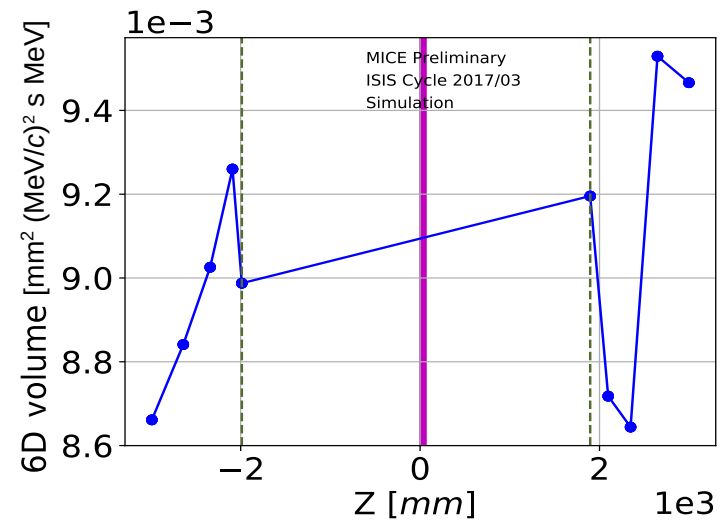

(b)

Figure 4.18. Evolution of the density (a) and volume (b) of the $2^{\text {nd }}$-percentile contour. The changes in density and volume from $z \approx-1.8 \times 10^{3} \mathrm{~mm}$ to $z \approx 1.8 \times 10^{3} \mathrm{~mm}$ tracker reference plane (vertical dashed green lines) are the results of $6 \mathrm{D}$ heating or reverse emittance exchange. 


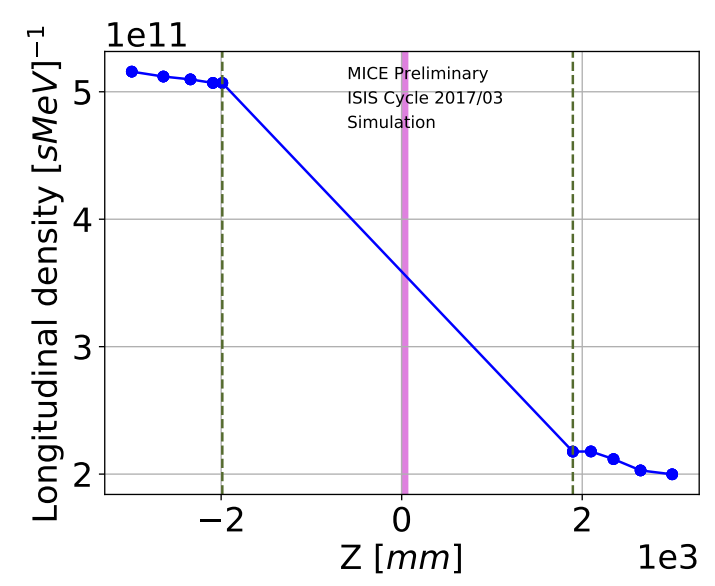

(a)

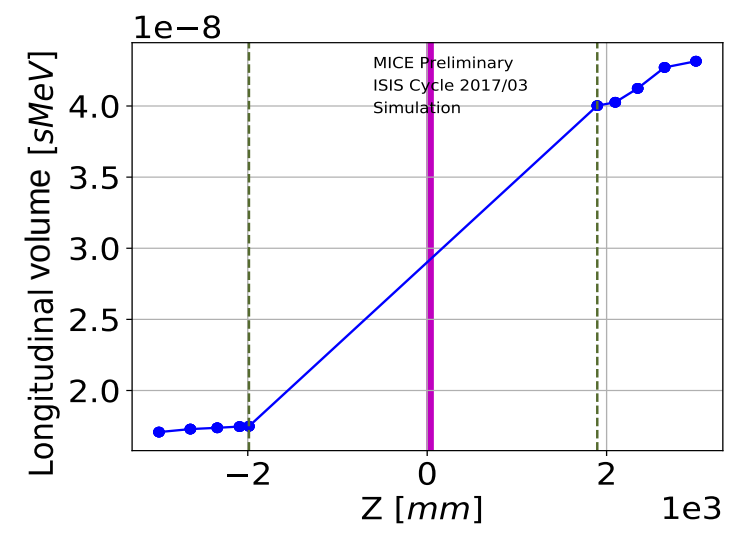

(b)

Figure 4.19. Evolution of the density (a) and volume (b) of the $24^{\text {th }}$-percentile contour for wedge simulation. The changes in density and volume from the upstream $\left(z \approx-1.8 \times 10^{3}\right.$ $\mathrm{mm})$ to downstream $\left(z \approx 1.8 \times 10^{3} \mathrm{~mm}\right)$ tracker reference plane (vertical dashed green lines) are the results of beam heating in the longitudinal direction. 


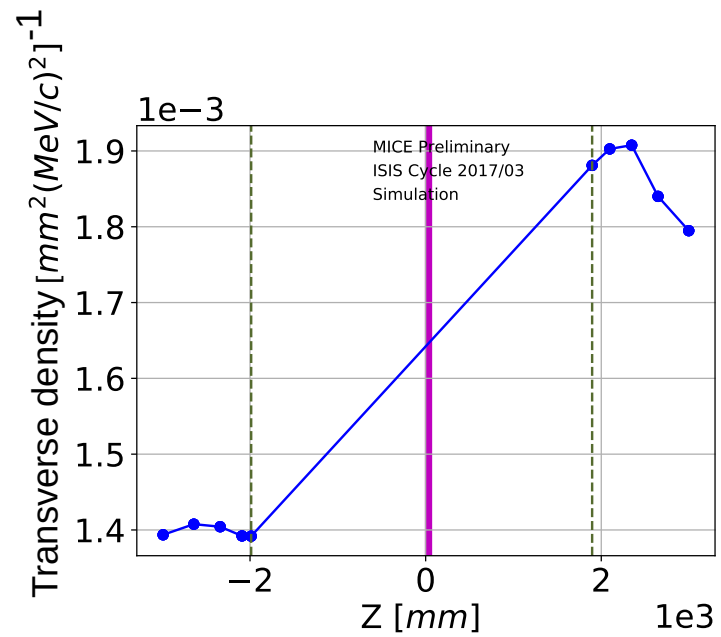

(a)

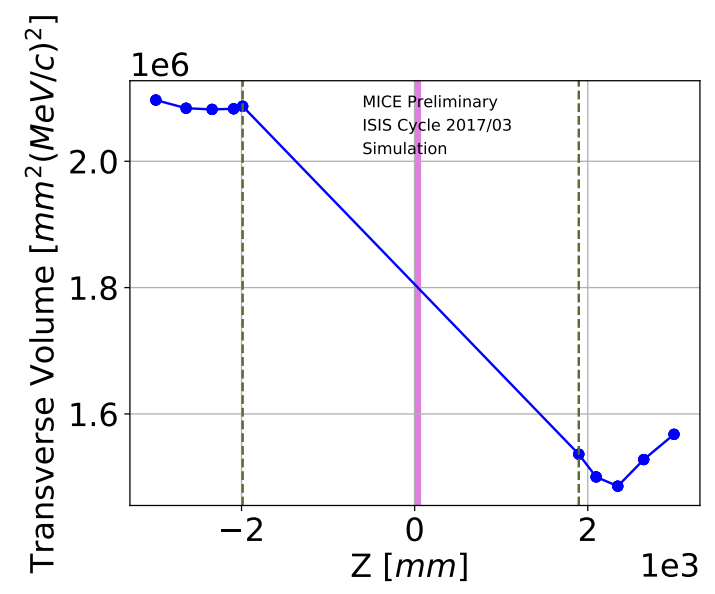

(b)

Figure 4.20. Evolution of the density (a) and volume (b) of the $9^{\text {th }}$-percentile contour for the wedge absorber simulation. The changes in density and volume from the upstream to downstream of the wedge (vertical dashed green lines) are the results of transverse beam cooling. 


\subsection{Summary}

The performance of the KDE technique was first studied in a quadrupole focusing channel, where (per Liouville's theorem) in the absence of material, the estimated density was conserved. The kernel density estimator was then applied to a simulated beam distribution traversing a matched solenoidal channel using a so-called MICE "baseline lattice" geometry; all the magnets were operating at nominal currents, and a flat $\mathrm{LiH}$ absorber was placed in the channel. No muon transmission loss was observed with these settings, and using the KDE technique, transverse beam cooling was demonstrated in the form of an increase in the beam core phase-space density and reduction in the beam core phase-space volume. Then, the kernel density estimator was applied to a beam distribution traversing a lattice where the magnet currents deviated from those of the "baseline lattice": the matching coils downstream of the $\mathrm{LiH}$ absorber were turned off, causing the beam distribution to become mismatched at the exit of the absorber and the muon transmission loss to drop below $90 \%$ (about 85\%). Despite such mismatched optics (leading to a non-Gaussian beam distribution at the exit of the absorber), the estimated density using the KDE approach still demonstrated an increase in the core phase-space density and reduction in the core phase-space volume. The core phase-space evolution plots showed core density increase and core volume reduction; however, they did not provide a complete picture of the beam cooling signal: muons in the tail of the distribution migrating to the beam core. Therefore, for a complete analysis of the cooling signal, a data visualization approach should be selected such that an increase in the core density accompanied by a simultaneous reduction in the phase-space density in the beam periphery is shown. For this, a range of phase-space contour densities at their given beam radii (density versus average beam radius plots) was constructed, where the expected cooling signal (muons in the tail of the distribution migrating to the beam core) was observed. 
The same type of studies (as above) were done for a cooling channel containing a polyethylene wedge-shaped absorber. A wedge absorber is capable of exchanging the transverse cooling (as obtained with a flat $\mathrm{LiH}$ absorber) for longitudinal cooling. The kernel density estimator was used in studying the muon beam distribution traversing the wedge absorber, and both direct and reverse emittance exchange were observed. 


\section{CHAPTER 5 NOVEL APPLICATION OF KDE IN DATA}

MICE has collected a total of 350 million particle triggers ${ }^{31}$ with various beamline, magnetic, and absorber configurations (Fig. 5.1). In this chapter, two different absorber configurations are studied: a $65 \mathrm{~mm} \mathrm{LiH}$ absorber and a 45 degree polyethylene wedge absorber. The beam setting with which the data were collected is $6-140$, and the magnet coil currents are the same as those in Table 4.5.

The MICE data structure has multiple "layers." A "spill" (each dip of the MICE target into the proton beam) is the topmost layer; in each spill, raw signals (raw data) from trackers and PID detectors is collected by the MICE data acquisition (DAQ) system. For physics analysis (e.g., cooling measurements based on the position and momentum of each muon), the raw data need to be reconstructed using a set of offline detector-specific reconstruction routines; the reconstruction routines convert the raw signals into measurements corresponding to the properties of a given particle traversing the PID detectors and trackers. The reconstructed data is the final layer of the MICE data structure used for beam cooling studies.

A typical MICE data file is written as a ROOT ntuple [55]; Figure 5.2 [56] shows an example of the structure within such a data file. The ntuple consists of tree entries, each entry corresponding to a step in the reconstruction process (e.g., tracker digit, cluster, space-point, reconstructed track) in one spill. The MC branch in the tracker data structure is the (simulated) Monte Carlo (MC) truth; it is reconstructed (referred to as $\mathrm{MC}$ recon) using the same reconstruction routines as those

${ }^{31} \mathrm{~A}$ trigger is a criterion used to decide which detector signals (from particles interacting in the detector) to record when only a fraction of the total can be recorded (due to limitations in data storage capacity). 


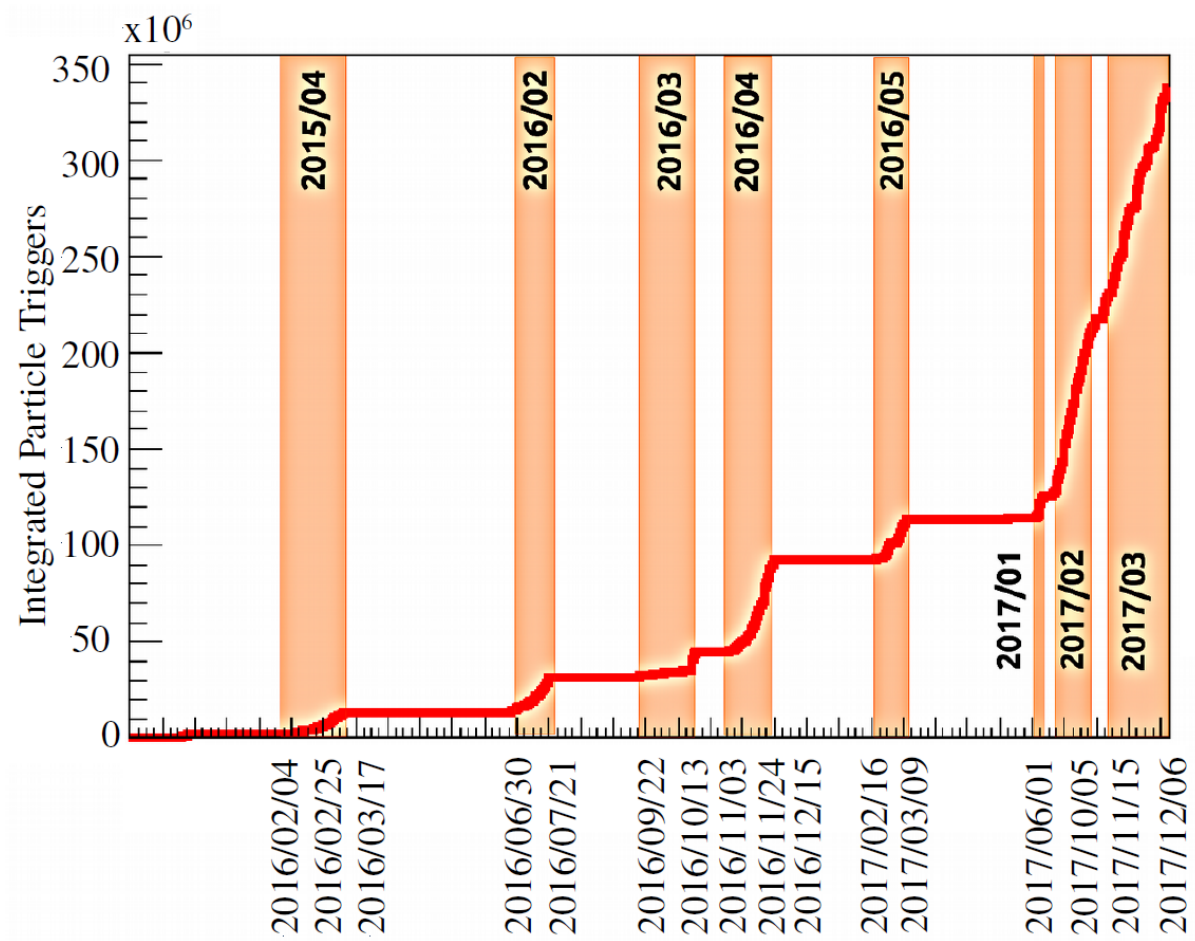

Figure 5.1. The total number of particle triggers as recorded by the ToF1 detector in the course of MICE operation [54].

used to reconstruct MICE raw data. For a comparison of the experimental data with simulation, the $\mathrm{MC}$ recon data are compared with the experimental data (more on this comparison in Sections 5.1 and 5.2). As shown in Figure 5.2, the final product in the tracker reconstruction process is the "SF (Scintillating Fiber) Helical Kalman Track" which contains entries for each muon's position and momentum coordinates along each spatial direction, $x, y$, and $z$. This is the information used for the beam cooling analysis in Sections 5.1 and 5.2. 


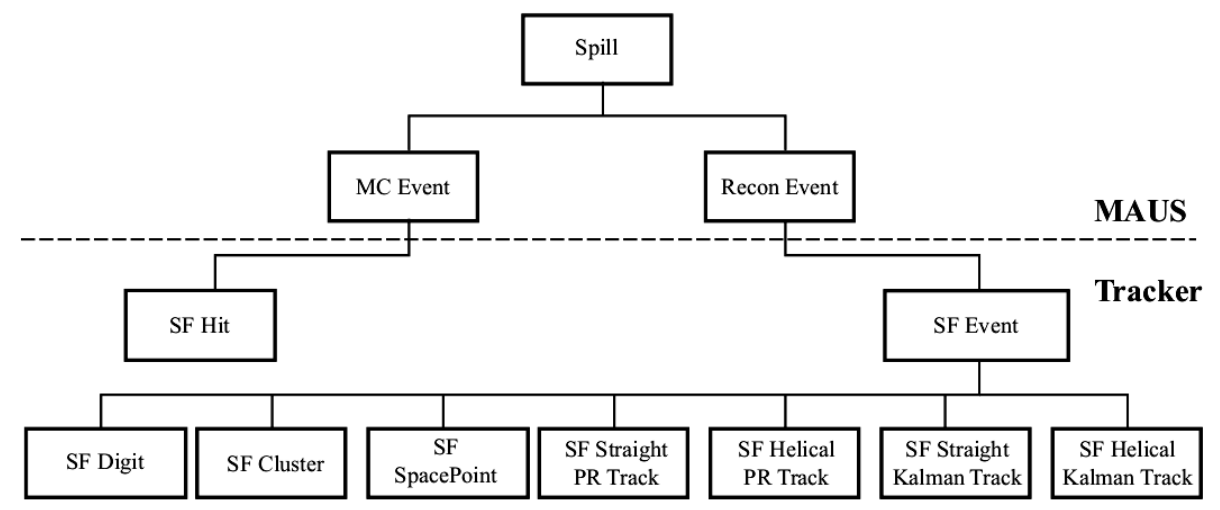

Figure 5.2. The typical MICE data structure [56].

\subsection{LiH Flat Absorber Analysis}

The MICE LiH data were collected with a $65 \mathrm{~mm}$ flat $\mathrm{LiH}$ absorber placed inside the AFC module. The coils in the SS and the AFC modules were operating at the current values specified in Table 4.5; only the downstream matching coil 1 was turned off for this dataset (Fig. 4.9). The input beam and reference momentum values were respectively $6 \mathrm{~mm}$ and $140 \mathrm{MeV} / c$ (6-140 setting) and data were collected using the "pionic beam" setting (Fig. 2.12).

Two types of beam selection cuts are applied to the LiH data: particleidentification (PID) and data quality. The purpose of the PID cut is to minimize beam impurity by rejecting contaminating particles such as electrons and pions from the MICE beam. The purpose of the data quality cut is to minimize reconstruction inefficiency. ${ }^{32}$ In the case of the PID cuts, muon ToF (time-of-flight from ToF0 to ToF1) and momentum range are selected; Table 5.1 lists the ranges of muon ToF and momenta used in the selection. The momentum versus ToF distributions before and after the cut are displayed in Figures 5.3 and 5.4. To implement the data quality cuts, only those muons that are within the "tracker fiducial area" and have a "ToF0

${ }^{32}$ The reconstruction efficiency quantifies how well a reconstruction algorithm reconstructs the raw detector signals. 
Table 5.1. List of PID cuts applied to the LiH experimental and simulated data along with their respective selected values.

\begin{tabular}{lc}
\hline \hline Cut Type & Cut Values \\
\hline ToF & 28 to $32 \mathrm{~ns}$ \\
Upstream Momentum & 130 to $150 \mathrm{MeV} / c$ \\
Downstream Momentum & 80 to $200 \mathrm{MeV} / c$ \\
Fiducial Area & $r_{\perp}>150 \mathrm{~mm}$ \\
\hline
\end{tabular}

and ToF1 single spacepoint" and "single track" are selected. The purpose of a ToF single spacepoint cut is to remove muons that enter the experiment within the same trigger window and leave more than one spacepoint in ToF0 and ToF $1 .^{33}$ The purpose of a single track cut is to isolate muons that consistently hit all tracker planes. The single track cut does not remove muons that scrape downstream of the absorber (scraping muons). Since the scraping muons make it past the absorber, they should be included in the beam cooling sample. Therefore, the single track cut is used only to discard muons that have intermittent hits on the tracker stations (e.g., producing a signal in tracker stations $5,3,2,1$ but not in 4$)$. The tracker fiducial area cut is used to discard muons that are not correctly reconstructed in the trackers and are found outside of the tracker transverse radius $\left(r_{\perp}=\sqrt{x^{2}+y^{2}}>150 \mathrm{~mm}\right)$.

Two types of data were analyzed and compared: the reconstructed Monte Carlo truth (muon position and momentum coordinates first recorded by virtual detectors and then reconstructed using the MAUS reconstruction routine) referred to as $\mathrm{MC}$ recon and the (experimental) data (containing the raw detector signals that are reconstructed offline by the MAUS reconstruction routine). The upstream and downstream distributions (at the upstream and the downstream tracker reference planes)

${ }^{33}$ Each ToF detector consists of two perpendicularly oriented scintillator slabs along the $x$ and $y$ projections; a ToF spacepoint is formed when particles hit both ToF slabs. 

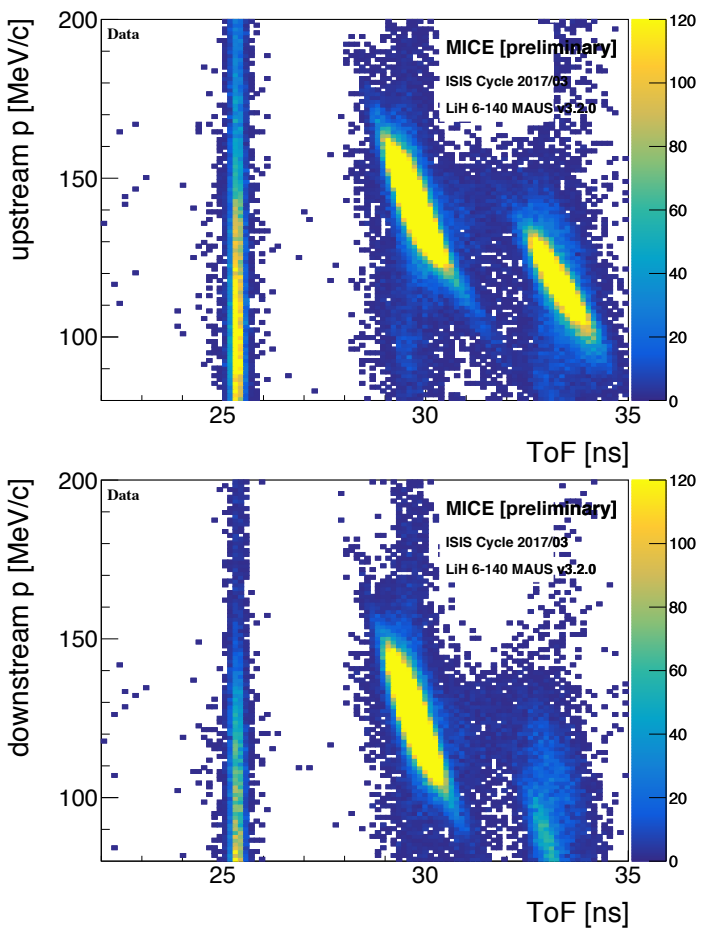
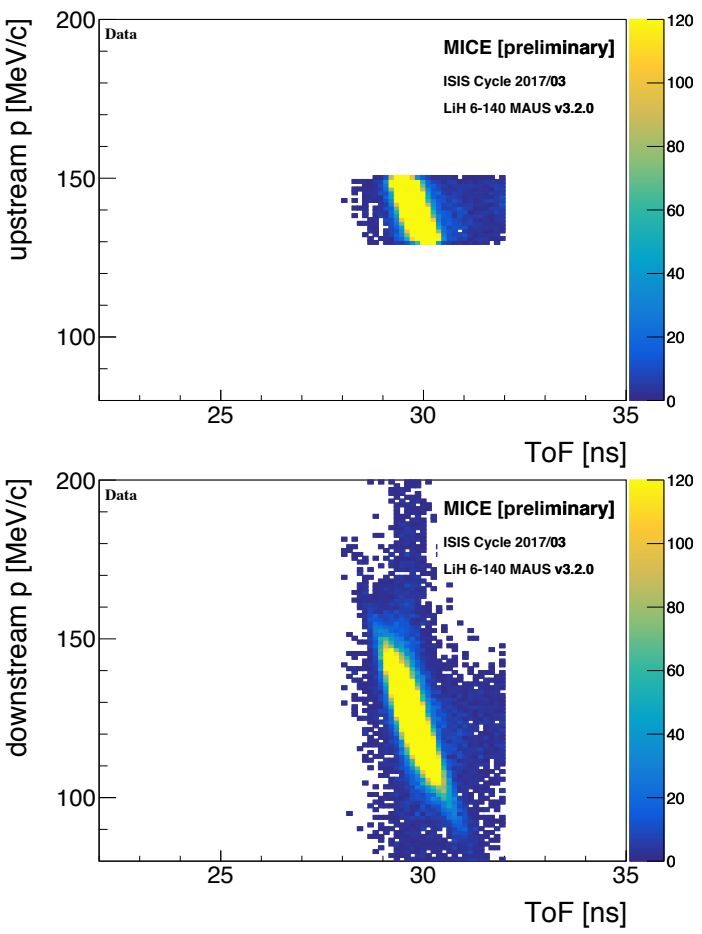

Figure 5.3. ToF versus upstream (upper plots) and downstream (lower plots) tracker momentum before (leftmost plots) and after (rightmost plots) the PID cuts applied on MICE data; these cuts are used to select the ToF and momenta corresponding to a muon beam in data. The cuts follow the MICE analysis convention. Note that the colorbars are the counts, normalized to the upstream total muon count (hence the same maximum count in all plots). 

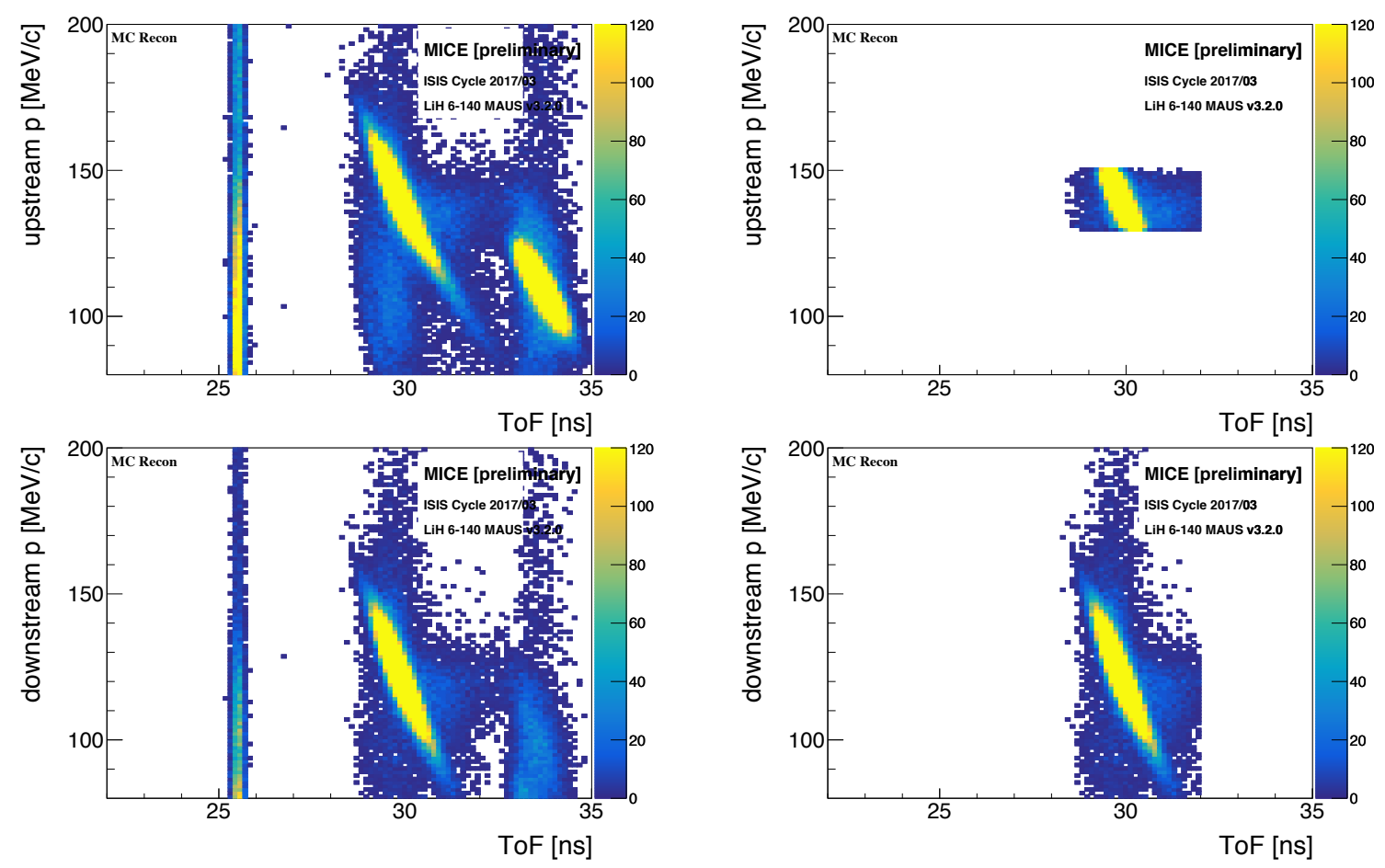

Figure 5.4. ToF versus upstream (upper plots) and downstream (lower plots) tracker momentum before (leftmost plots) and after (rightmost plots) the PID cuts applied on MC recon sample; these cuts are used to select the ToF and momenta corresponding to a muon beam in MC recon. The cuts follow the MICE analysis convention. 

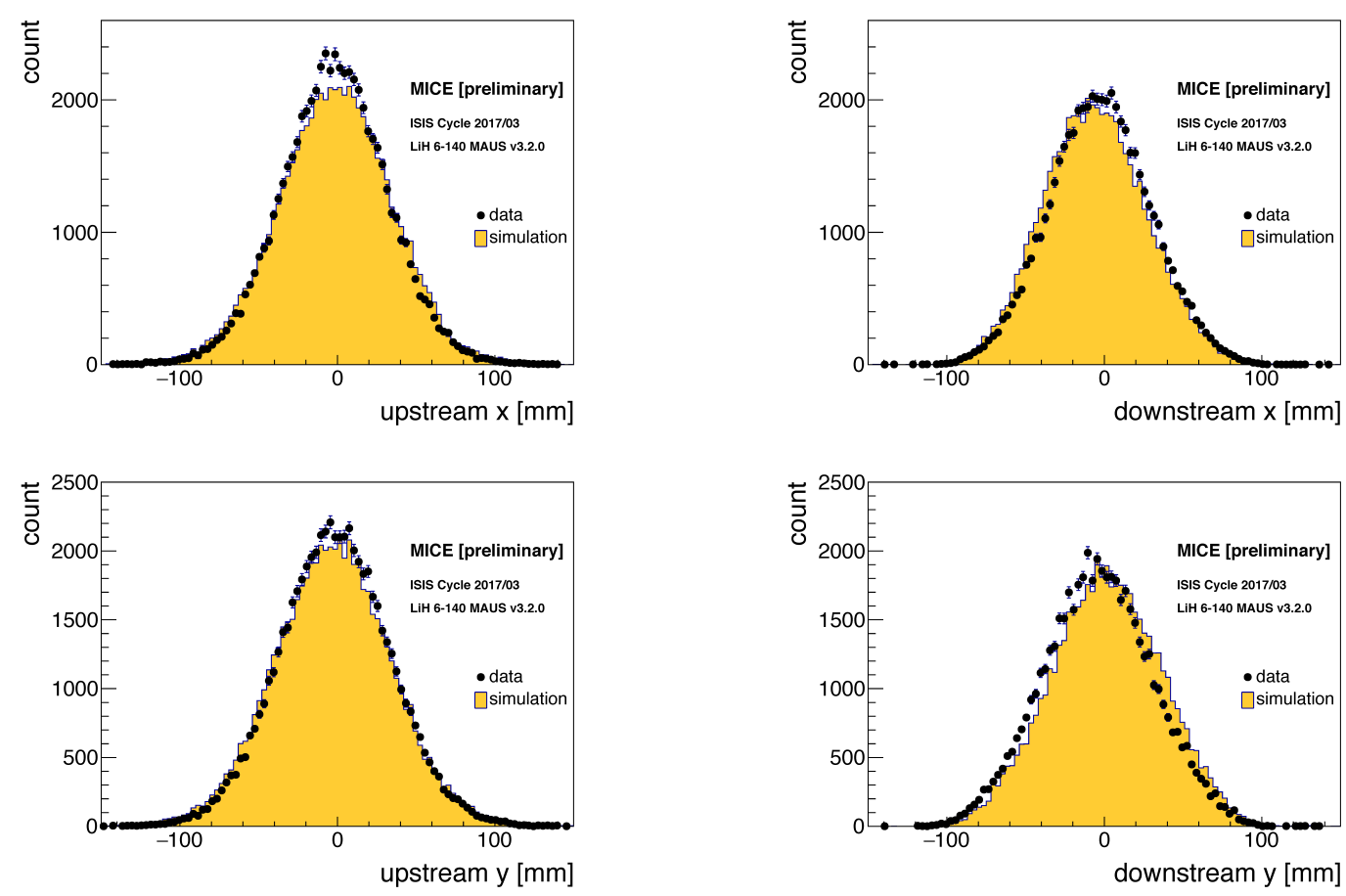

Figure 5.5. Comparison of the distributions in $x$ (upper plots) and $y$ (lower plots) before (left) and after (right) the $\mathrm{LiH}$ absorber (at the upstream and the downstream tracker reference planes) in simulation (MC recon) and (MICE experimental) data.

for each of the transverse coordinates in data and MC recon are shown in Figures 5.5 and 5.6; they illustrate a qualitative agreement between simulation and data.

Because of the correlations between the transverse coordinates (introduced by the solenoidal fields of the SS and AFC), a one-dimensional distribution of each coordinate is not sufficient for demonstrating beam cooling. The phase-space plots in Figures 5.7, 5.8, 5.9, and 5.10 can be used as a qualitative measure of the beam cooling: each plot represents a phase-space projection. The points are the individual muons and the colors the estimated (4D) densities (obtained using the KDE technique). The core estimated densities in the experimental and simulated phase-space projections are observed to increase as a result of transverse beam cooling. This is apparent from an increase in color brightness (colors are the estimated densities in units of $\left[\mathrm{mm}^{2}\right.$ $\left.\left.(\mathrm{MeV} / c)^{2}\right]^{-1}\right)$ towards the beam center in the downstream phase-space projections (Figs. 5.8 and 5.10). In addition, a qualitative comparison between data (Figs. 5.7 

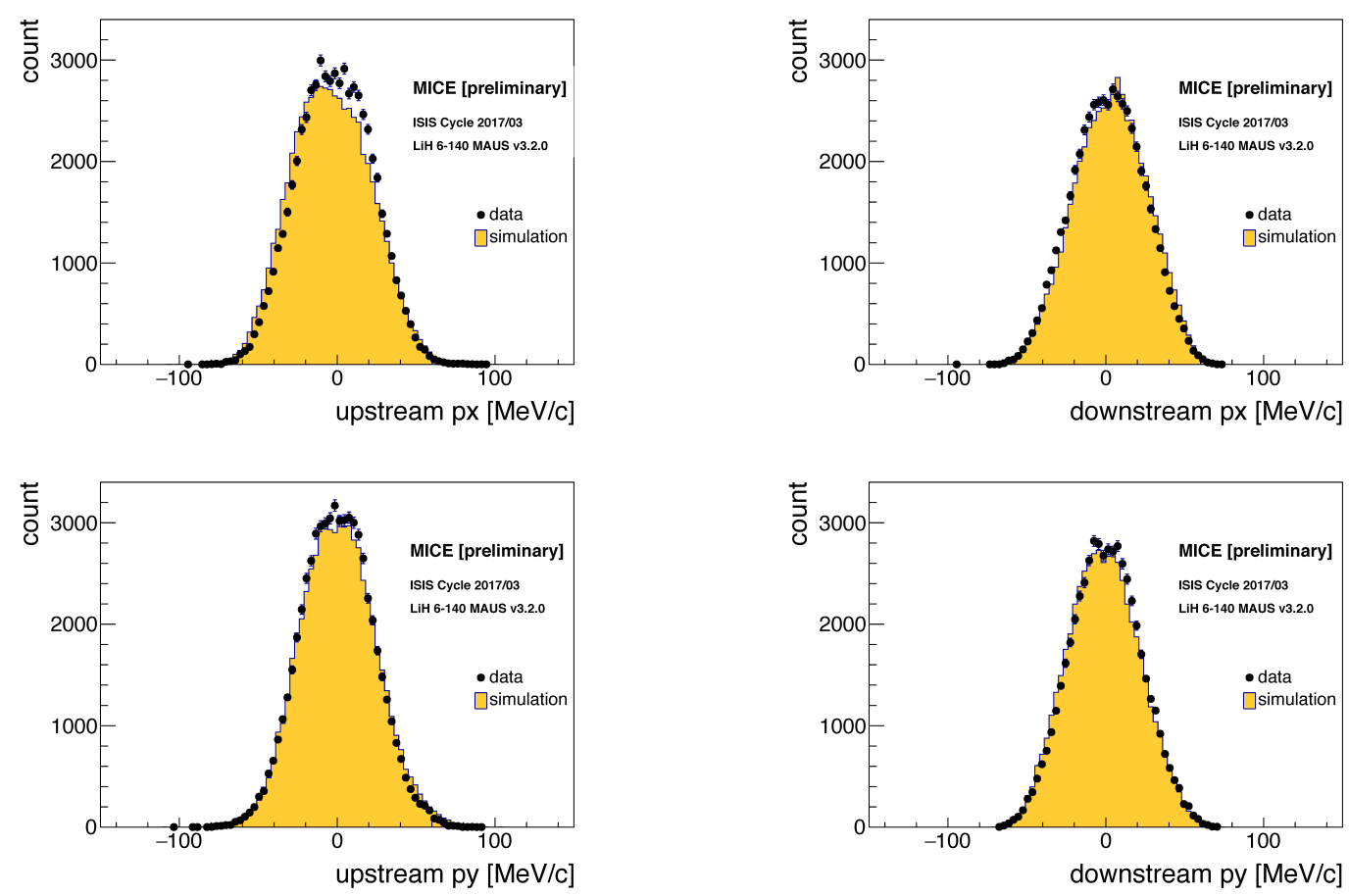

Figure 5.6. Comparison of the distributions in $p_{x}$ (upper plots) and $p_{y}$ (lower plots) before (left) and after (right) the $\mathrm{LiH}$ absorber in simulation (MC recon) and (MICE experimental) data.

and 5.8) and MC recon (Figs. 5.9 and 5.10) reveals that the MC recon upstream distributions tend to occupy larger volumes than the upstream beam distributions in data. This discrepancy between data and simulation could be due to the simulated MICE beamline not being fully optimized in MAUS.

A more quantitative measure of beam cooling can be done by plotting the density versus the average beam radius. In the transverse plane, the plots are shown in Figures 5.11a and 5.12a. Both data and MC recon demonstrate transverse beam cooling (a $12 \%$ increase in data at the core beam radius and a $19 \%$ increase in $\mathrm{MC}$ recon at the core radius); core density at the low beam radii (located at $20[\mathrm{MeV} / c$ $\mathrm{mm}]^{1 / 2}$ ) increases as a result of muons migrating from the beam periphery to beam core. As discussed earlier, in Figures 5.7-5.10, the upstream MC recon distribution occupies a larger volume than the upstream data distribution. This causes discrepancies in the density estimates and transmission (91\% in data and $87 \%$ in MC recon) 

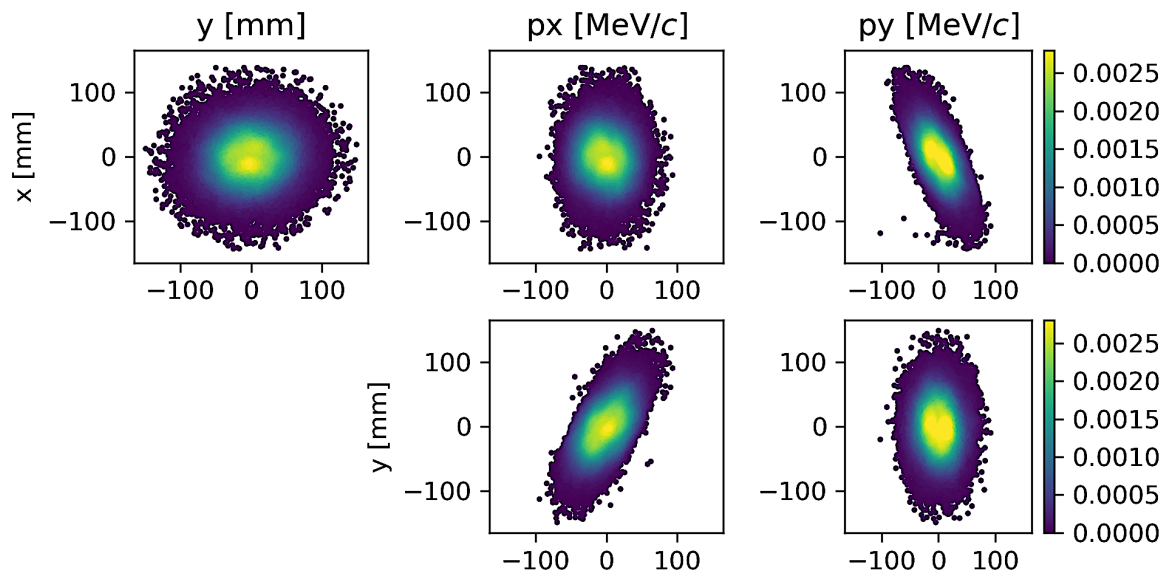

MICE [preliminary] ISIS cycle 2017/03 LiH Data MAUS v3.2.0

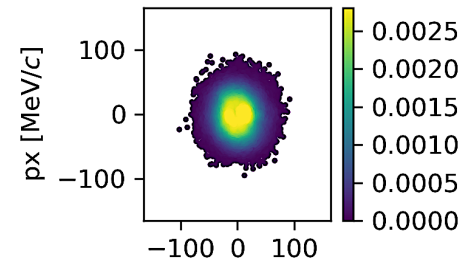

Figure 5.7. Phase-space plots of the upstream data sample.
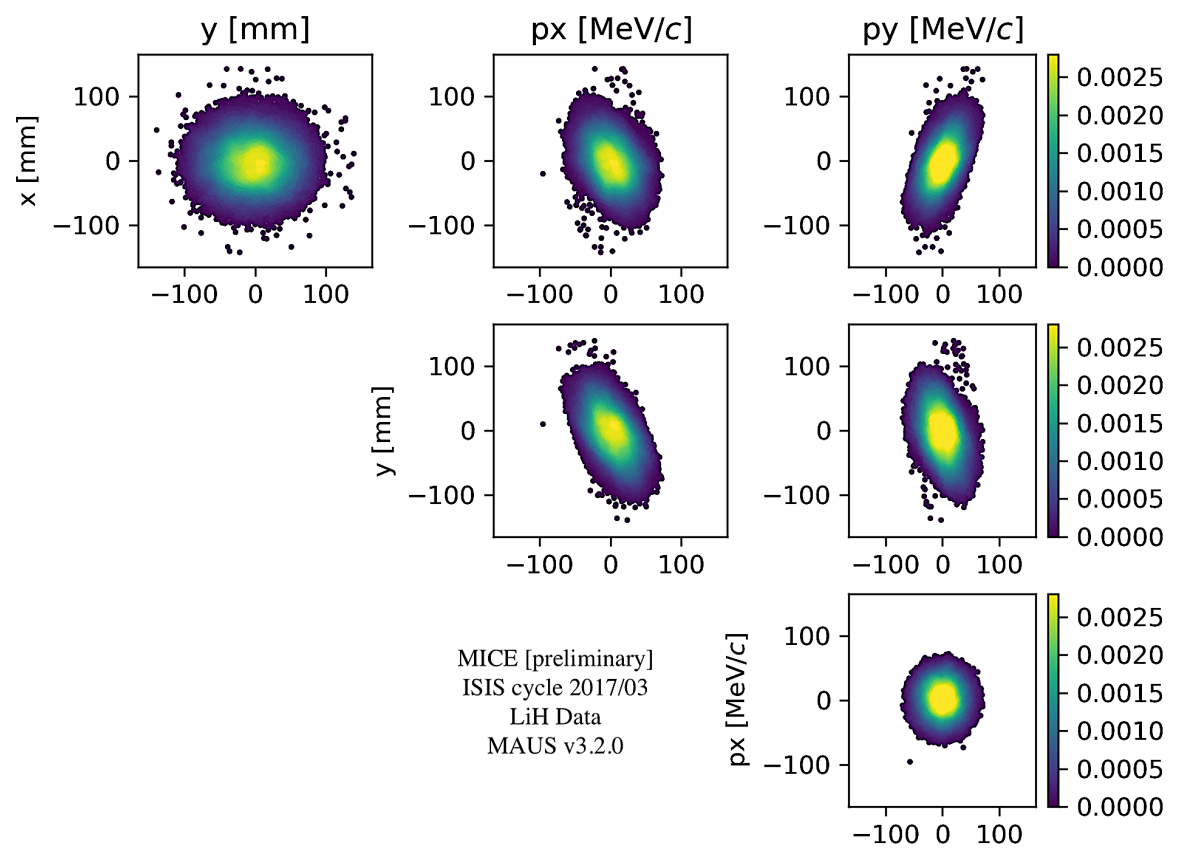

Figure 5.8. Phase-space plots of the downstream data sample. 

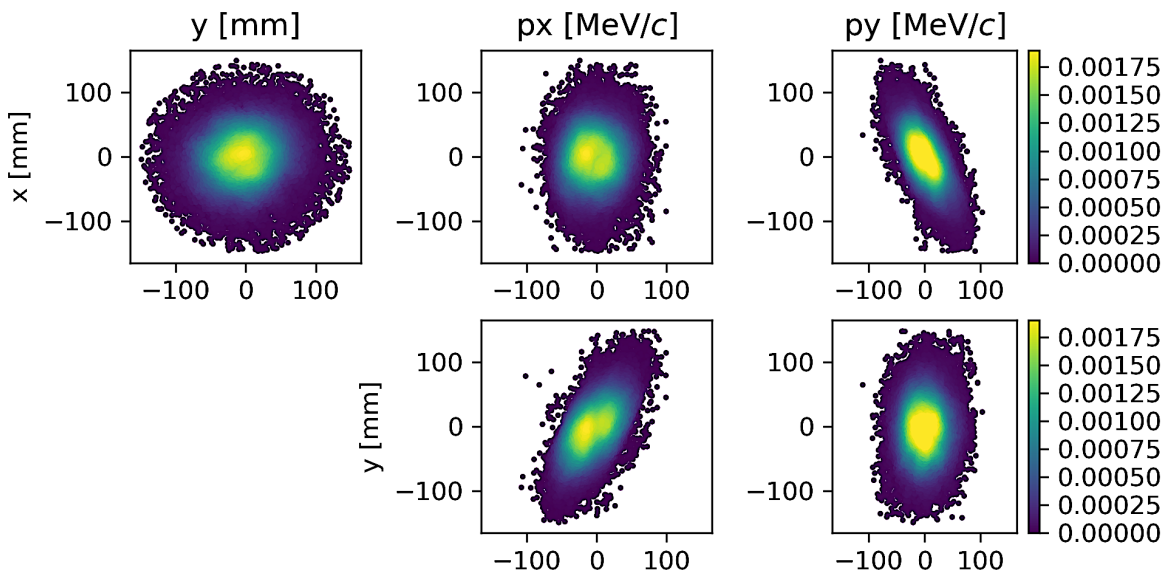

MICE [simulation] ISIS cycle 2017/03 $\mathrm{LiH}$

MAUS v3.2.0

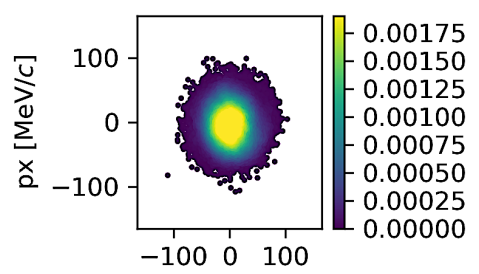

Figure 5.9. Phase-space plots of the upstream MC recon sample.
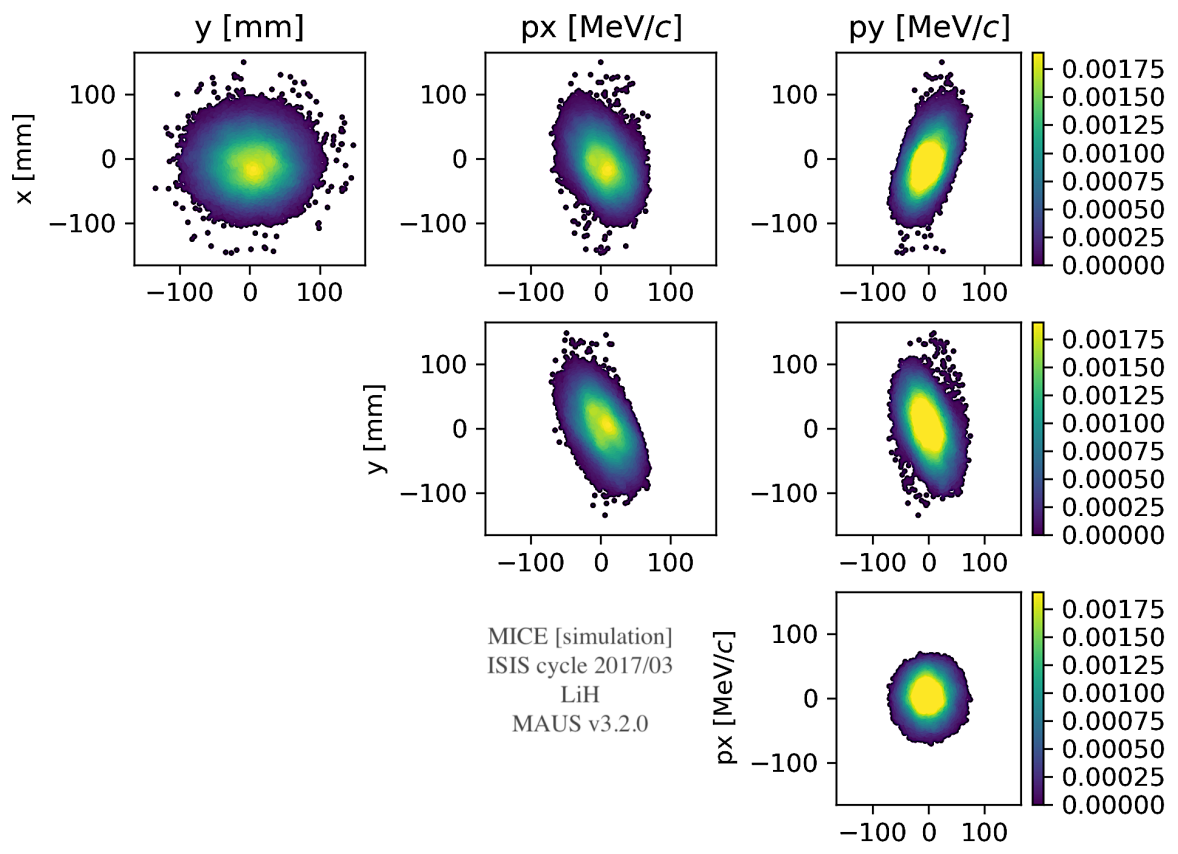

Figure 5.10. Phase-space plots of the downstream MC recon sample. 


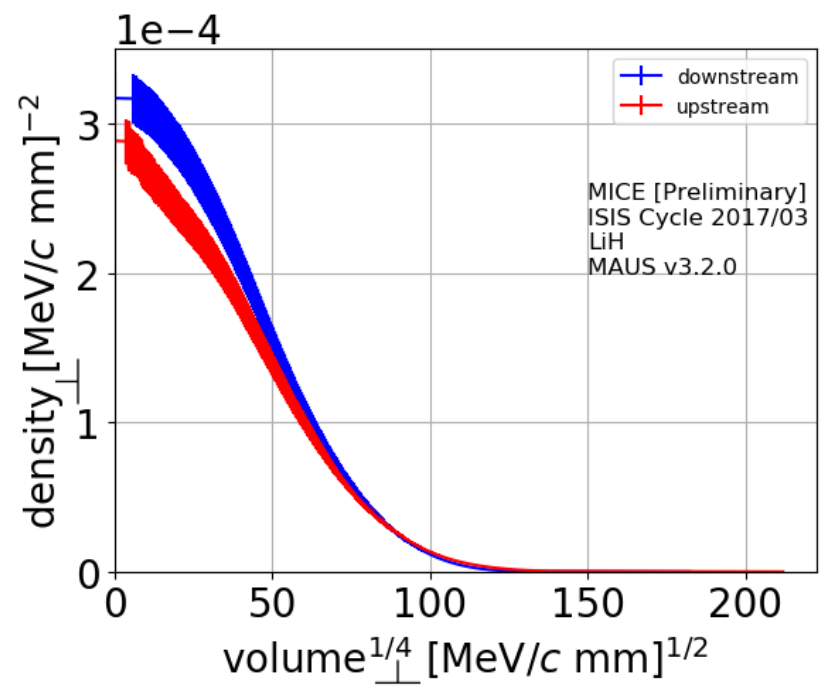

(a)

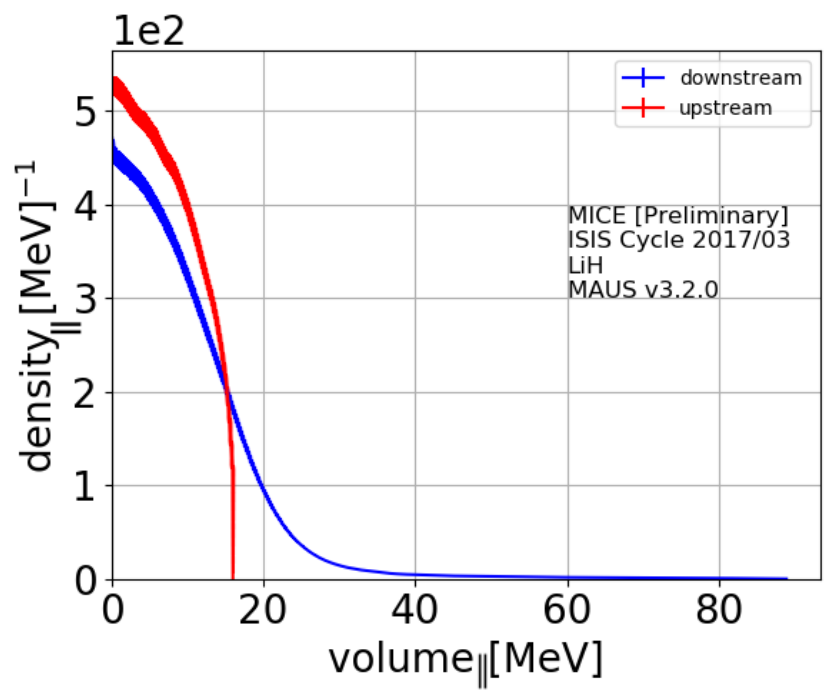

(b)

Figure 5.11. Contour density versus the average beam radius in the transverse (upper plot) and the longitudinal direction (lower plot) in data; the increase in the beam core density in the transverse direction and decrease in the core density in the longitudinal direction are the expected beam cooling signals from a flat absorber. 


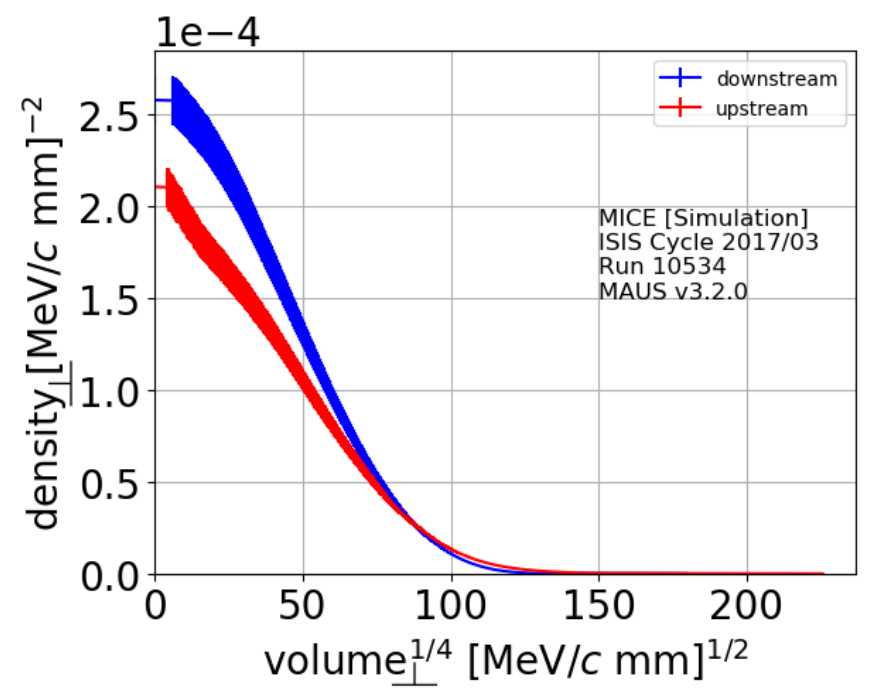

(a)

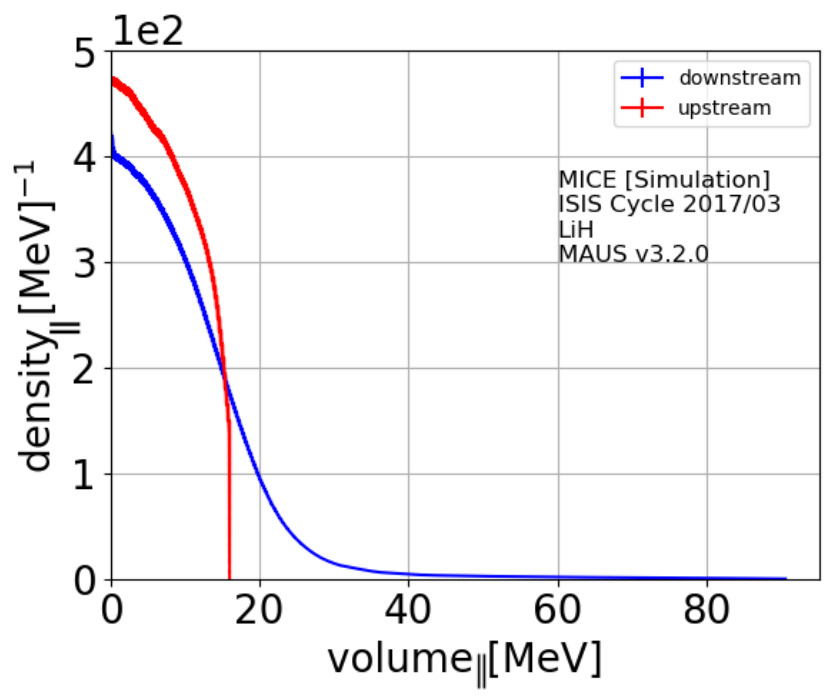

(b)

Figure 5.12. Contour density versus the average beam radius in the transverse (upper plot) and the longitudinal direction (lower plot) in $\mathrm{MC}$ recon; the increase in the beam core density in the transverse direction and decrease in the core density in the longitudinal direction are the expected beam cooling signals from a flat absorber. 
between data and $\mathrm{MC}$ recon samples; the $\mathrm{MC}$ recon emittance is farther from the equilibrium, which leads to a larger increase in density. In the longitudinal plane, whose coordinates are the muon energy $\left(E=\sqrt{p_{\mu}^{2}+m_{\mu}^{2}}\right)$, the density versus average beam radius plots (Figs. 5.11b and 5.12b) demonstrate heating; this is an expected result with a flat $\mathrm{LiH}$ absorber. The transverse density versus average beam radius plots are obtained using the KDE technique and the $\mathrm{MC}$ volume calculation routine (explained in Section 4.3); the density of each muon is estimated in the 4D phase space, and contours (a total of 1000 contours formed) of constant density are found. The volume of each contour is then calculated and the estimated density is plotted against the fourth root of the volume. The error bars in Figures 5.11a, 5.11b, 5.12a, and $5.12 \mathrm{~b}$ are obtained by dividing up the upstream and downstream muon samples into 10 subsamples of about 7000 muons each and taking the standard deviation of the 10 subsamples at each beam radius. 


\subsection{Polyethylene Wedge--shaped Absorber Analysis}

The wedge data were collected with a 45-degree polyethylene wedge absorber (with an on-axis length of $52 \mathrm{~mm}$ ). Figure 5.13 shows the orientation of the wedge in the AFC. Looking towards the downstream tracker, the thicker edge of the wedge was placed at larger $x$ values, and the thinner edge was located at $x=-62.77 \mathrm{~mm}$. The coils in the SS and AFC modules were operating at the values specified in Table 4.5; only the downstream matching coil 1 was turned off for this dataset (Fig. 4.9). The input beam and reference momentum values were $6 \mathrm{~mm}$ and $140 \mathrm{MeV} / c$ respectively (6-140 setting) and data were collected using the "pionic beam" setting (Fig. 2.12). The same type of PID cuts as for the LiH data (Section 5.1) were applied to the wedge dataset (Table 5.1). The data quality cuts are the same as those used in studying the LiH dataset (Section 5.1 and Table 5.1). The momentum versus ToF distributions at the upstream and the downstream tracker reference planes before and after the $\mathrm{PID}$ cuts for data and $\mathrm{MC}$ recon are displayed in Figures 5.14 and 5.15. Unlike the $\mathrm{LiH}$ data, the downstream momentum distributions in both $\mathrm{MC}$ recon and data are broader. This is a manifestation of the reverse emittance exchange effect: muons with higher $x$ values pass through the thicker edge of the wedge and lose more momentum, acquiring an $x-p_{z}$ correlation. This effect is best illustrated in Figures 5.16a, 5.16b, 5.17a, and 5.17b; the upstream longitudinal momentum and $x$ position (upper plots) are seen to acquire correlations downstream of the wedge absorber.

The individual transverse coordinates in data and $\mathrm{MC}$ recon are shown in Figures 5.18 and 5.19; according to these plots, there is a qualitative agreement between data and simulation. The longitudinal coordinate in $\mathrm{MC}$ recon and data are also shown in Figures 5.20a and 5.20b. Similar to the LiH data, only muon energies are used to construct the longitudinal coordinate; the time coordinate is not included because it is only necessary when an RF cavity is present in the cooling channel 


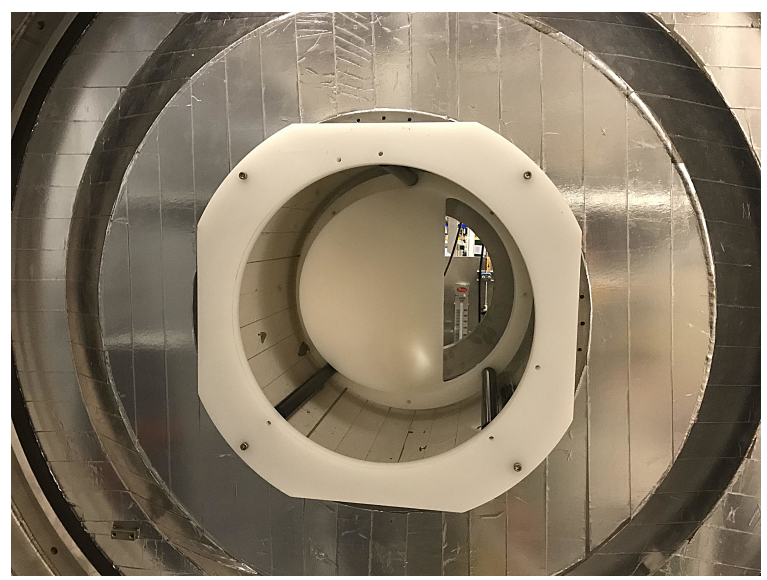

Figure 5.13. Photo of the wedge absorber installed inside the Focus Coil before insertion in the beam.
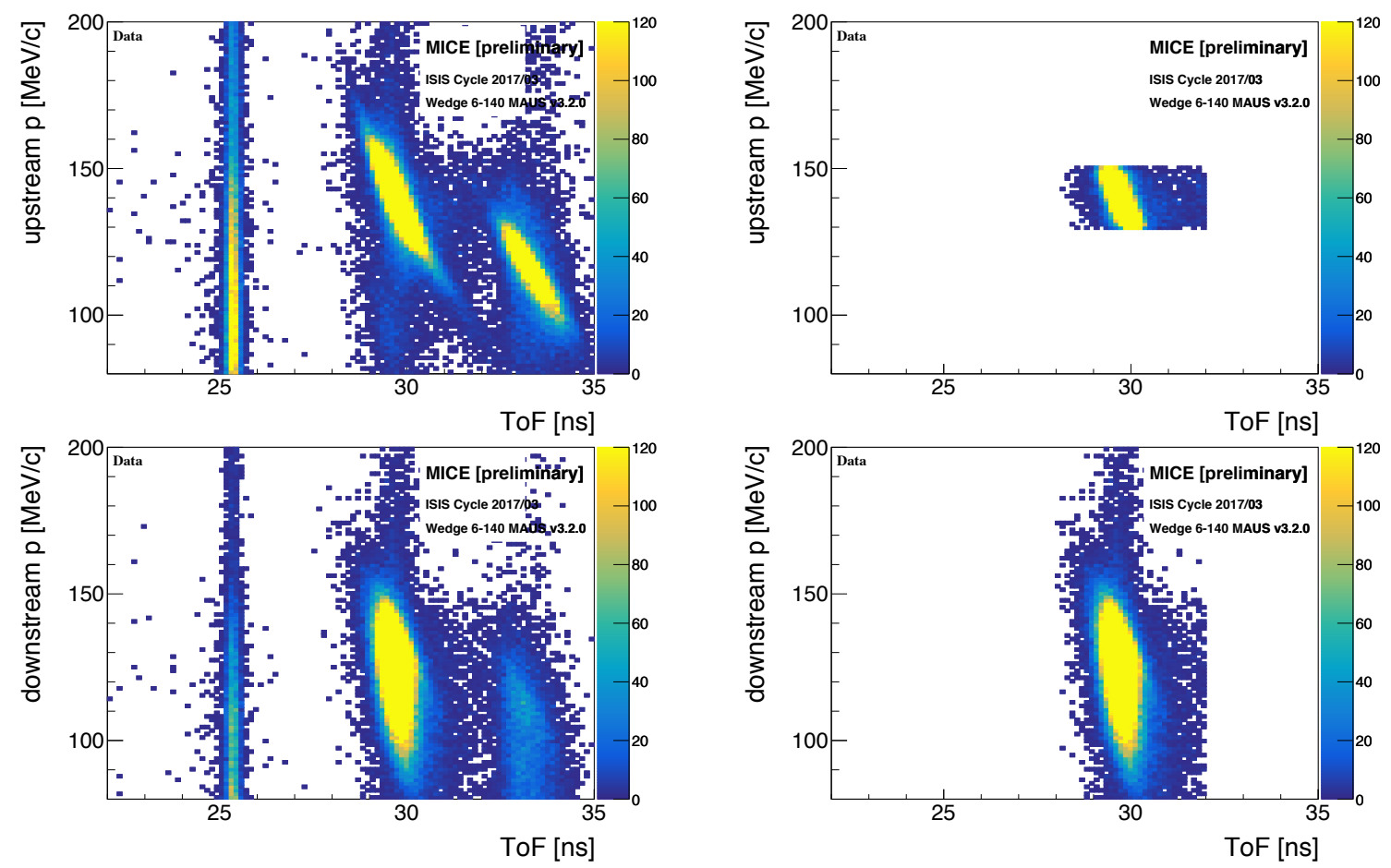

Figure 5.14. ToF vs. upstream (upper plots) and downstream (lower plots) tracker momentum before (left plots) and after (right plots) the PID cuts; these cuts are used to select the ToF and momenta corresponding to a muon beam in data and follow the MICE convention. 

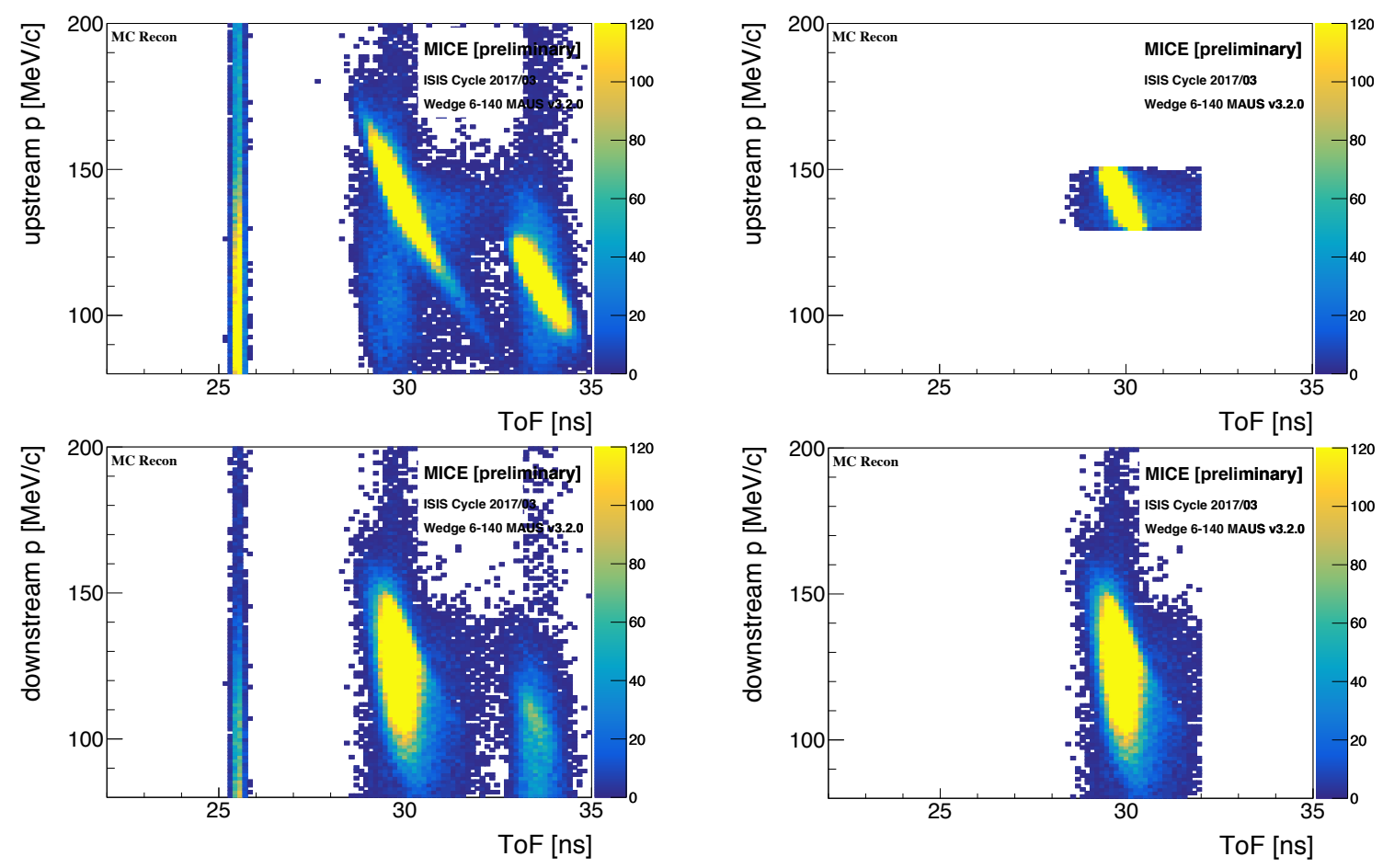

Figure 5.15. ToF vs. upstream (upper plots) and downstream (lower plots) tracker momentum before (leftmost plots) and after (rightmost plots) the PID cuts; these cuts are used to select the ToF and momenta corresponding to a muon beam in MC recon and follow the MICE analysis convention. 


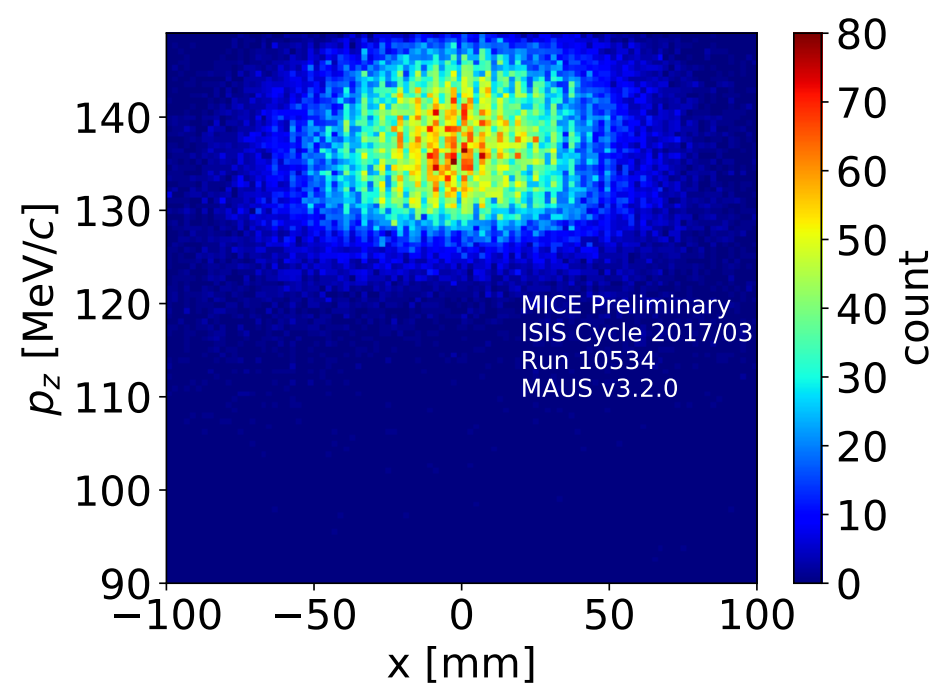

(a)

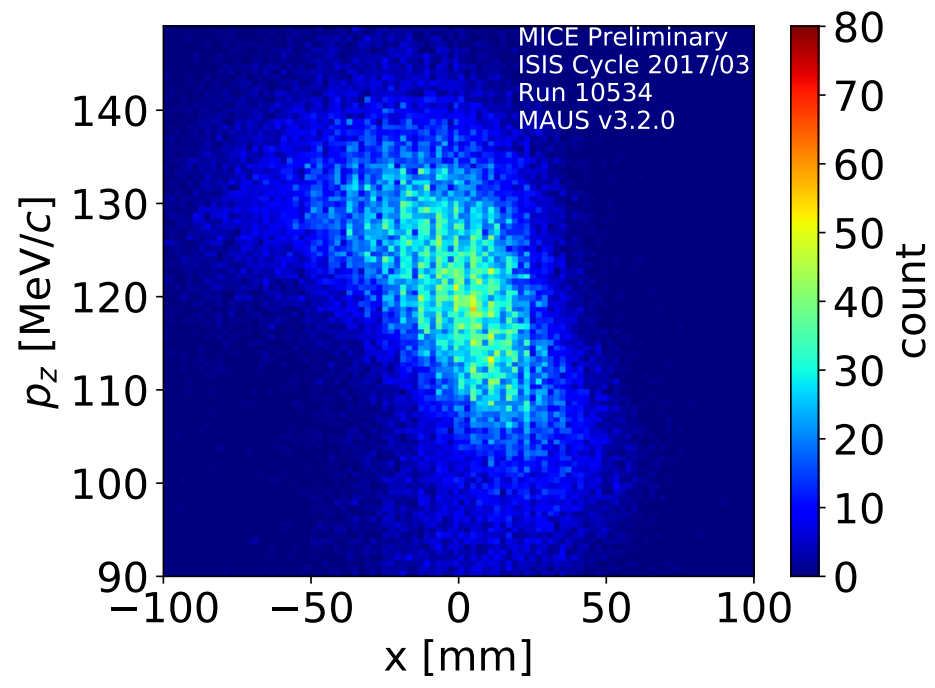

(b)

Figure 5.16. The wedge absorber introduces an $x-p_{z}$ correlation in the MICE experimental beam (data); this is the result of reverse emittance exchange. 


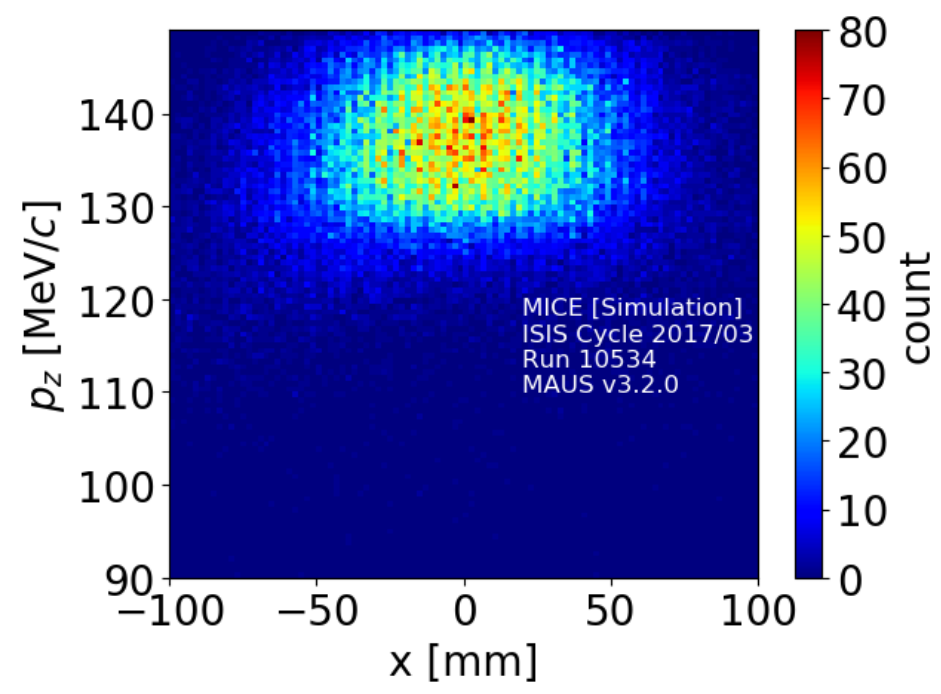

(a)

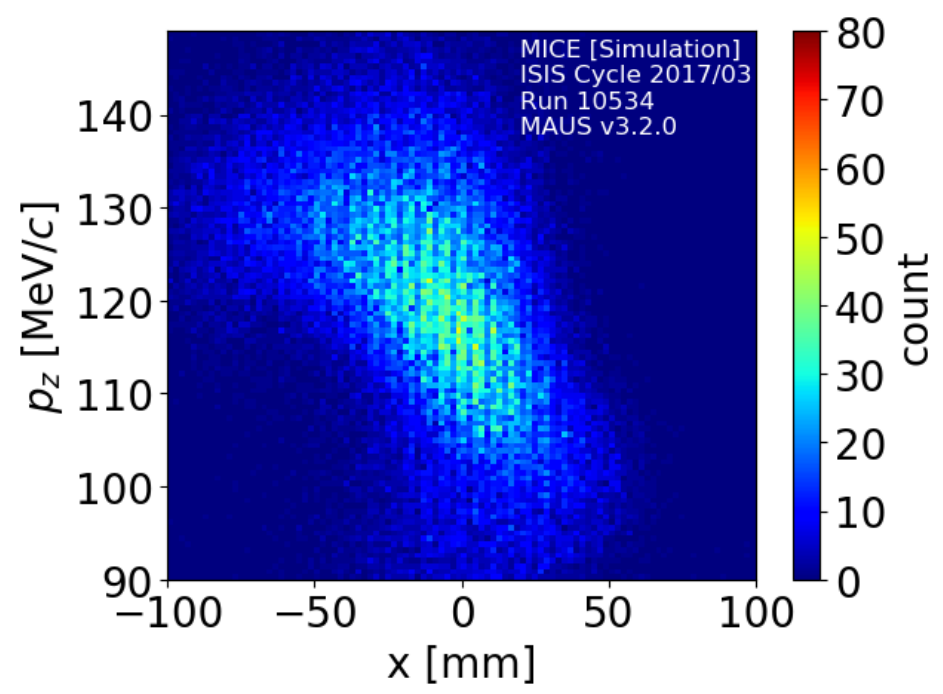

(b)

Figure 5.17. The wedge absorber introduces an $x-p_{z}$ correlation in the MICE simulated beam (MC recon); this is the result of reverse emittance exchange. 

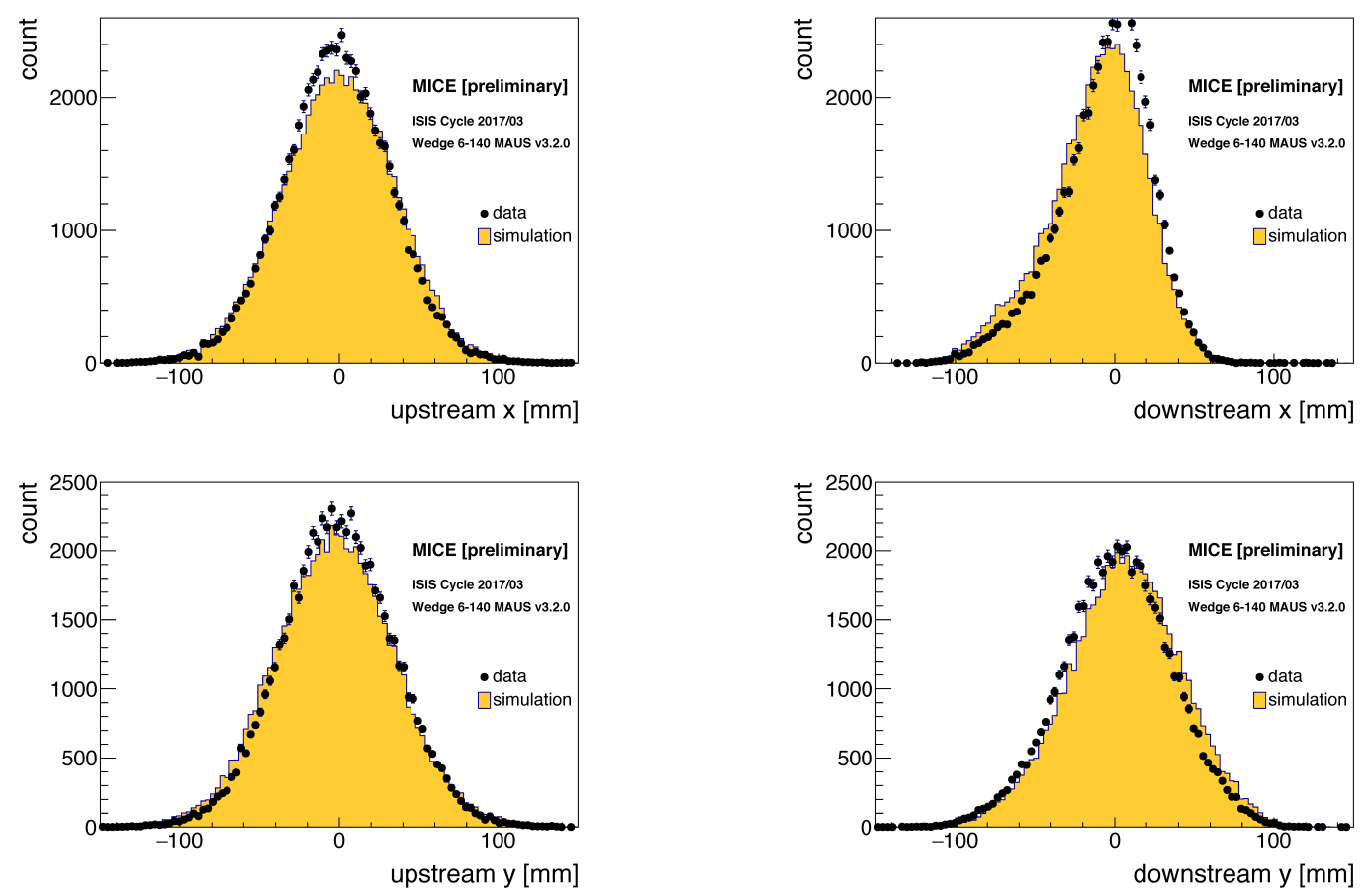

Figure 5.18. Comparison of the distributions in $x$ (upper plots) and $y$ (lower plots) before (left) and after (right) the $\mathrm{LiH}$ absorber (at the upstream and the downstream tracker reference planes) in simulation (MC recon) and (MICE experimental) data.
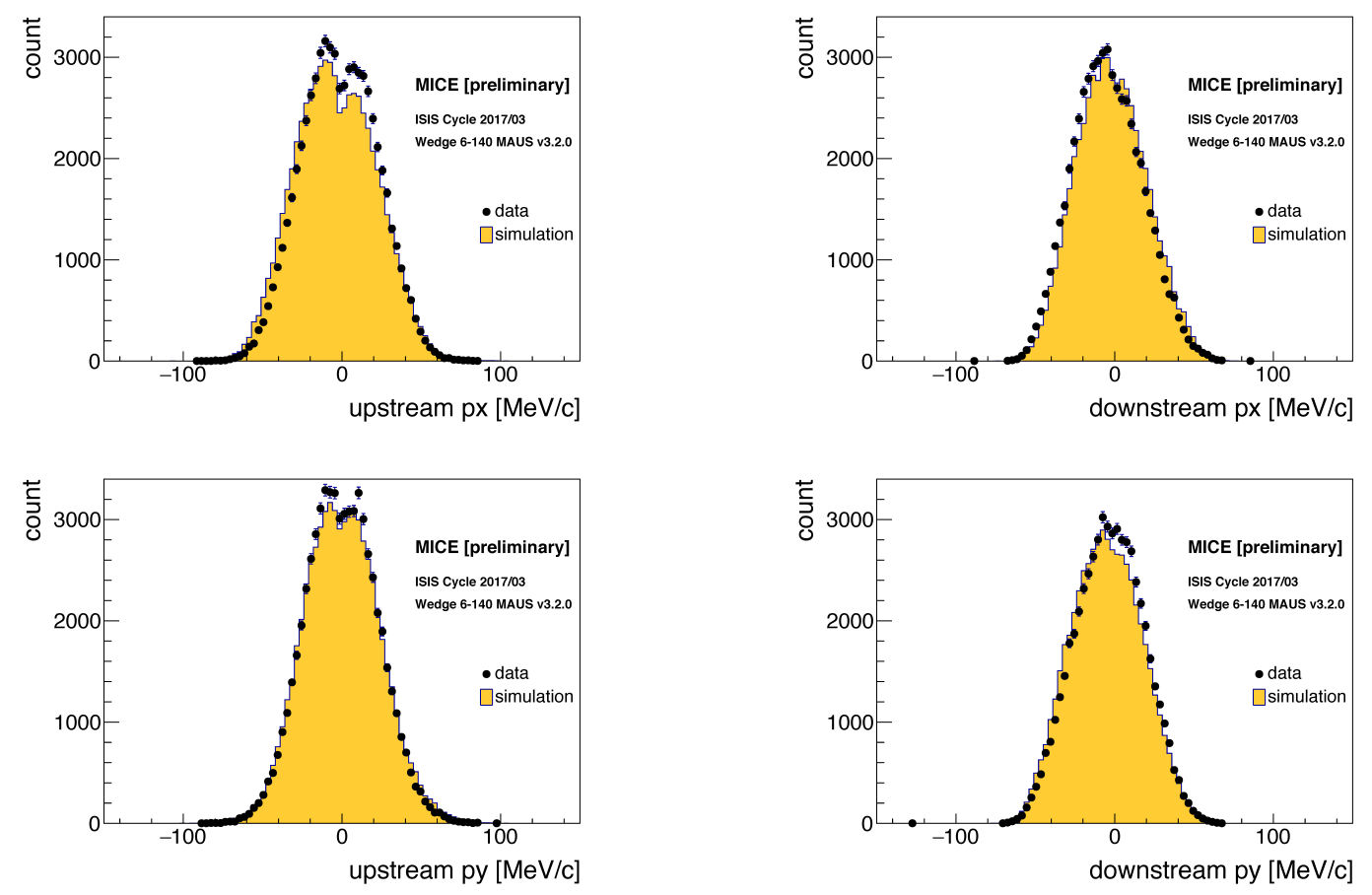

Figure 5.19. Comparison of the distributions in $p_{x}$ (upper plots) and $p_{y}$ (lower plots) before (left) and after (right) the $\mathrm{LiH}$ absorber in simulation (MC recon) and (MICE experimental) data. 


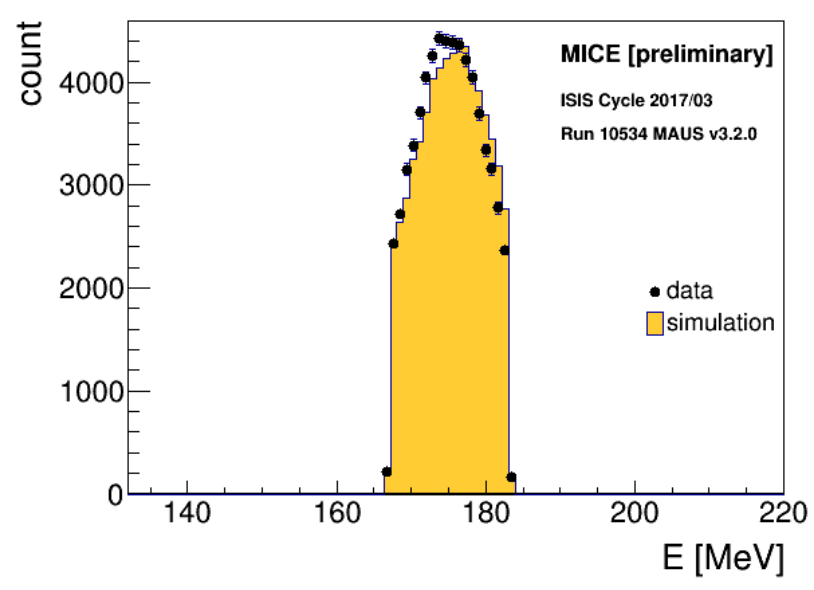

(a)

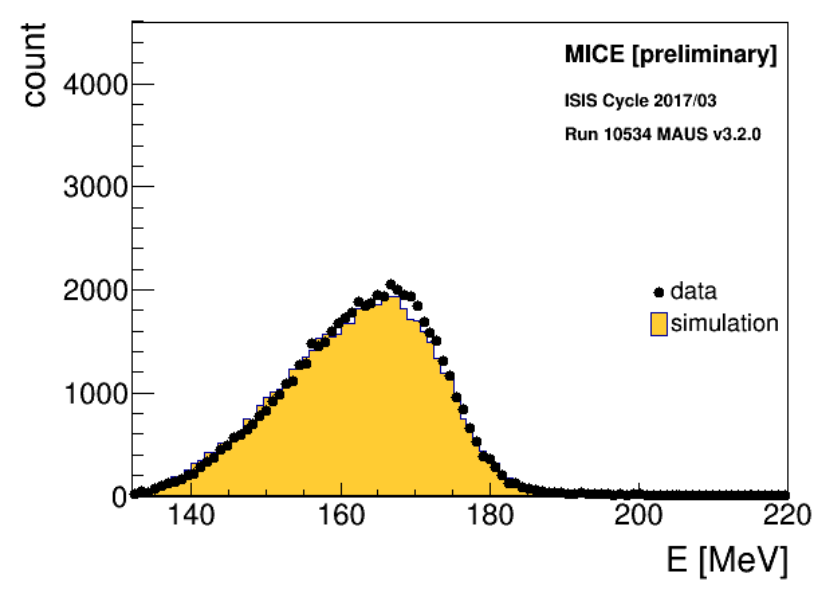

(b)

Figure 5.20. Comparison of the energy distributions upstream (upper plots) and downstream (lower plots) of the wedge absorber in data and MC recon. 


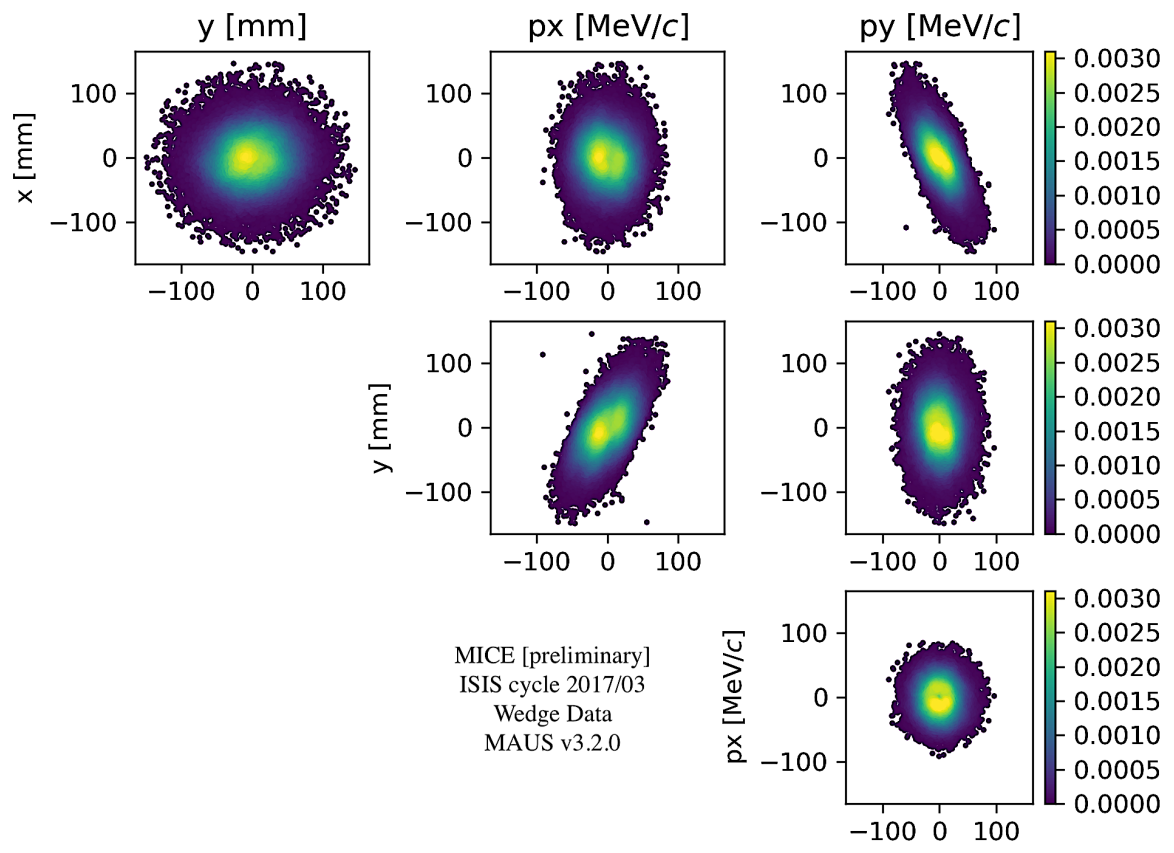

Figure 5.21. Phase-space plots of the upstream data sample.

(MICE final configuration, as shown in Figure 2.11, did not have an RF cavity in the channel).

Phase-space plots of $\mathrm{MC}$ recon and data are shown in Figures 5.21, 5.22, 5.23 , and 5.24; the points in these plots are the individual muons, and the colors the estimated densities (obtained using the KDE technique) in the four-dimensional phase space. Similarly to the LiH analysis, it can be observed that there is an increase in the core density as a result of transverse beam cooling; this is apparent from the increase in color brightness (estimated density in unit of $\left[\mathrm{mm}^{2}(\mathrm{MeV} / \mathrm{c})^{2}\right]^{-1}$ ) towards the beam core as the beam travels from the upstream tracker reference plane to the downstream (Figs. 5.22 and 5.24). Similarly to the LiH distributions, the MC recon upstream beam distribution tends to occupy a slightly larger area than the upstream beam distribution in data; this causes the transmission to differ in $\mathrm{MC}$ recon than data (transmission is $82 \%$ in $\mathrm{MC}$ recon and $87 \%$ in data). 

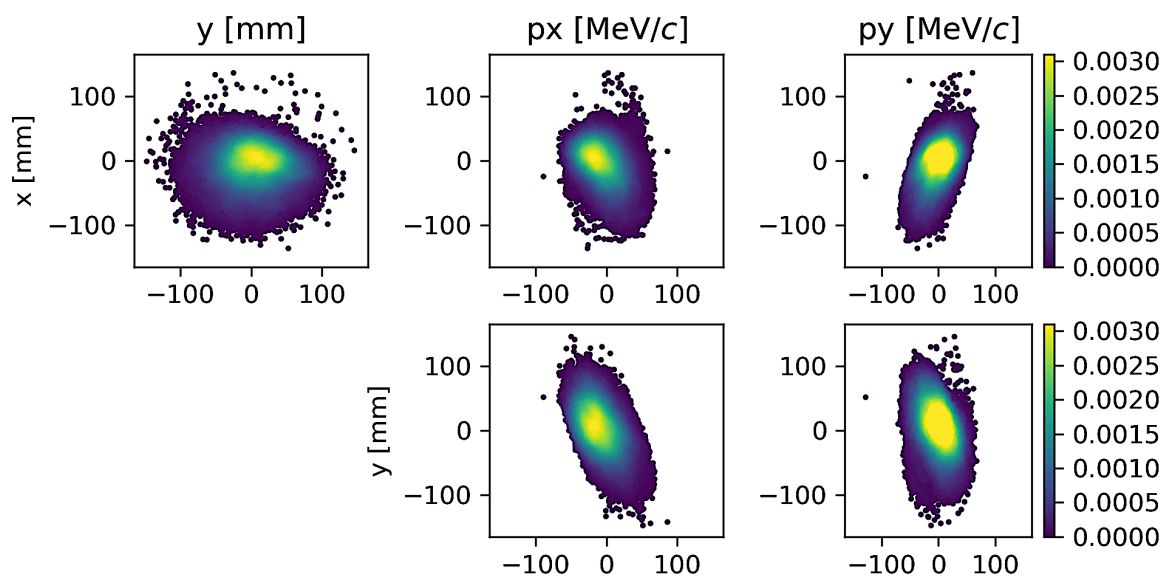

MICE [preliminary] ISIS cycle 2017/03 Wedge Data MAUS v3.2.0

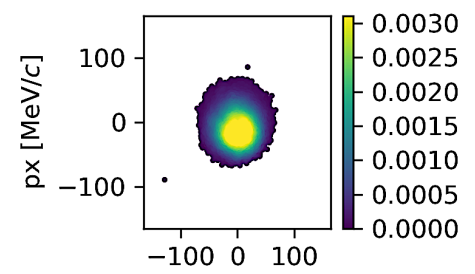

Figure 5.22. Phase-space plots of the downstream data sample.
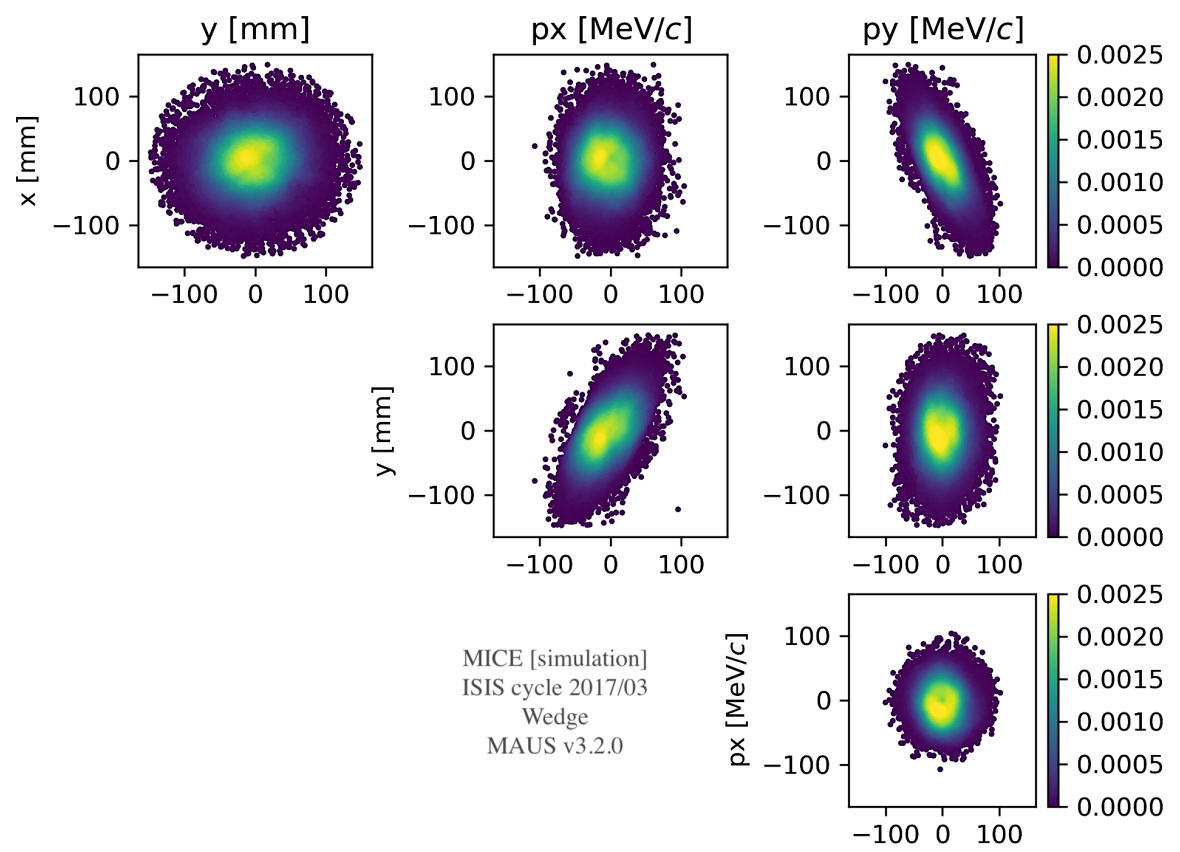

Figure 5.23. Phase-space plots of the upstream MC recon sample. 


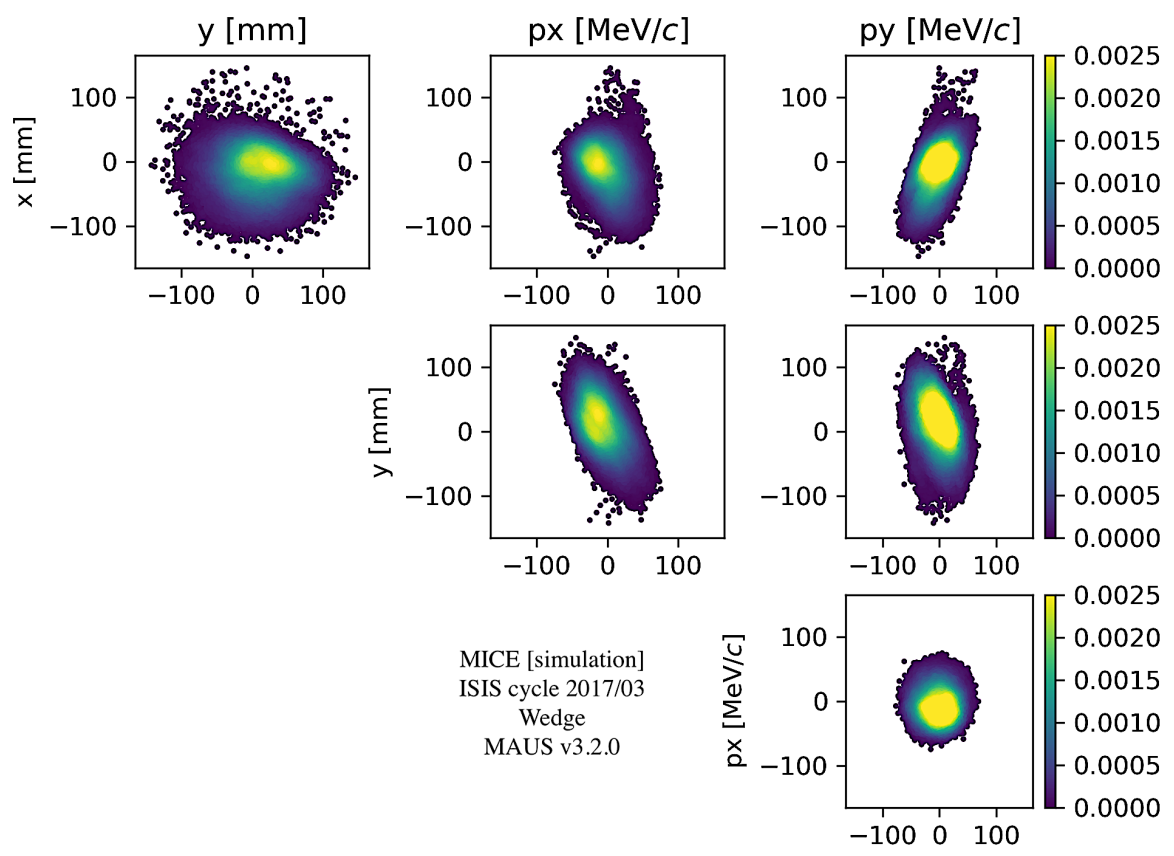

Figure 5.24. Phase-space plots of the downstream MC recon sample.

The plots of the transverse and longitudinal density versus average beam radius (the fourth root of volume) in data and simulation ( $\mathrm{MC}$ recon) are shown in Figures 5.25a, 5.25b, 5.26a, and 5.26b; these plots are obtained using the KDE technique and the MC volume calculation routine (explained in Sections 3.2 and 4.3). In the transverse plane, the density of each muon is estimated in the $4 \mathrm{D}$ phase space, and contours (a total of 1000 contours are formed) of constant density are found using the $\mathrm{MC}$ volume calculation technique. The density of each contour is then is plotted against the fourth root of the volume. The error bars in Figures 5.25a, $5.25 \mathrm{~b}, 5.26 \mathrm{a}$, and $5.26 \mathrm{~b}$ are obtained by dividing up the upstream and downstream muon samples into 10 subsamples of about 7000 muons each and taking the standard deviation of the 10 subsample estimated densities at each beam radius. Both data and MC recon distributions shown in Figures 5.25a, 5.25b, 5.26a, and 5.26b display transverse beam cooling and longitudinal beam heating. Cooling is the result of the migration of muons from the beam periphery to the beam core. As shown in Fig- 


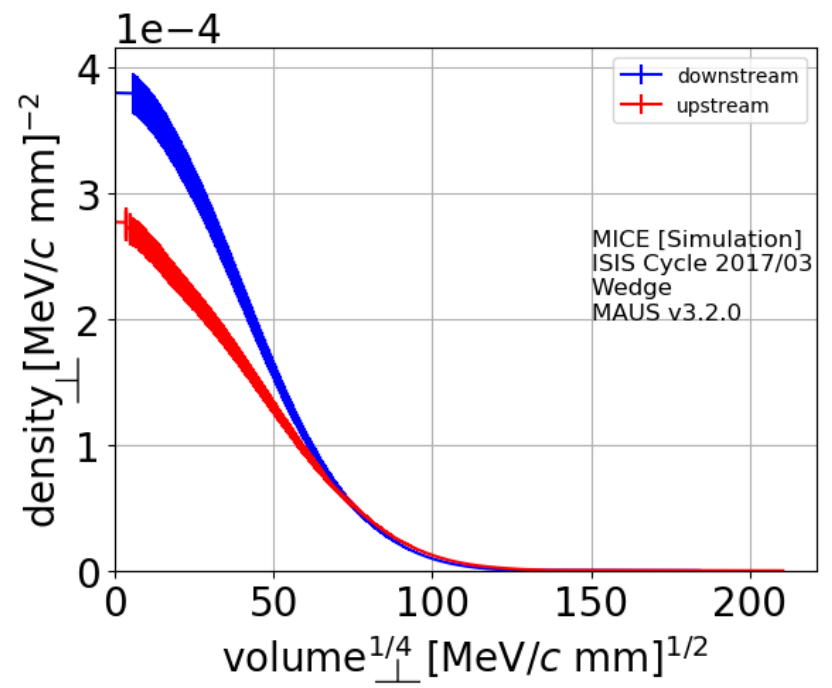

(a)

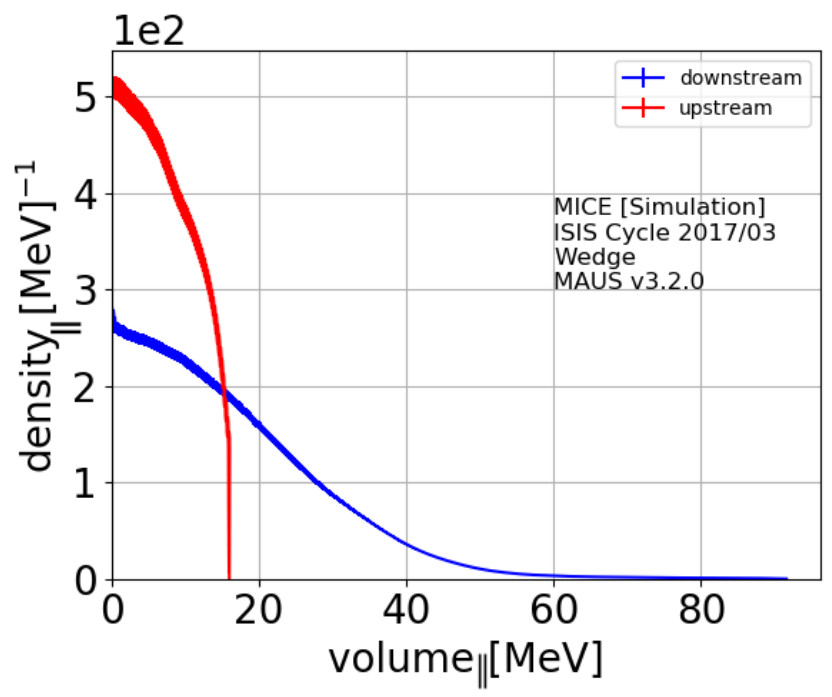

(b)

Figure 5.25. Contour density versus the average beam radius in the transverse plane (upper plot) and longitudinal plane (lower plot) in data; the increase in the beam core density in the transverse plane and decrease in density in the longitudinal plane are the expected beam cooling signals with a wedge absorber. 


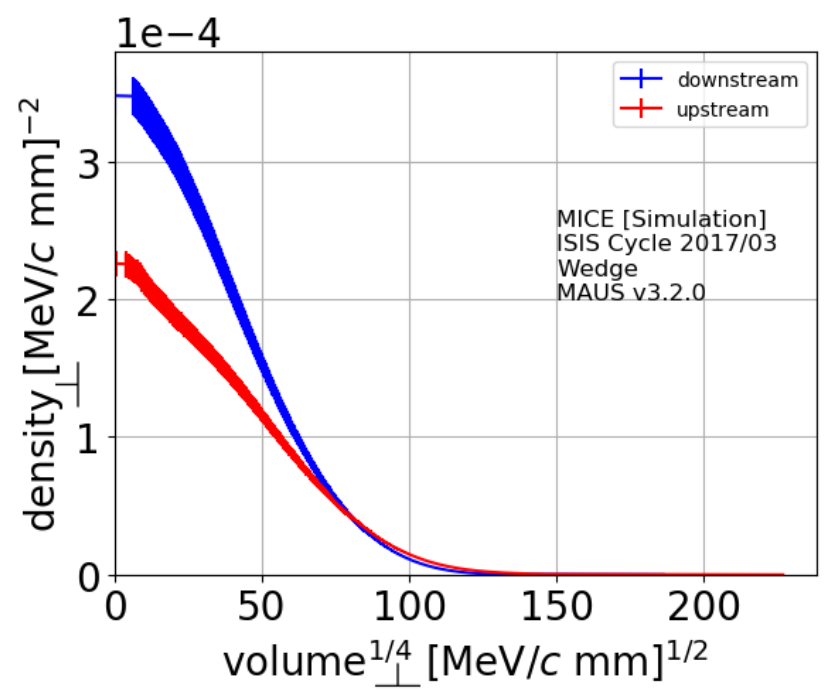

(a)

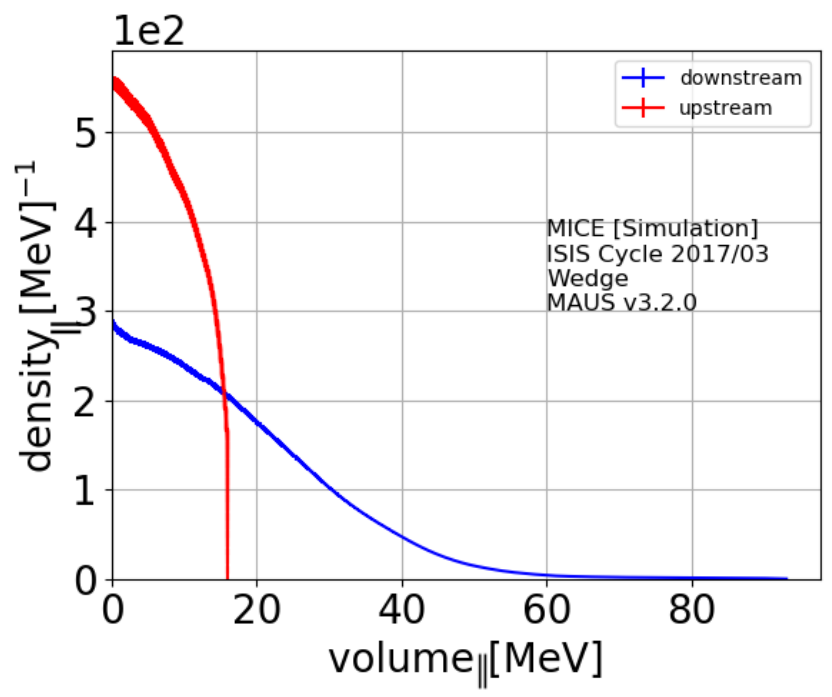

(b)

Figure 5.26. Contour density versus the average beam radius in the transverse plane (upper plot) and longitudinal plane (lower plot) in $\mathrm{MC}$ recon; the increase in the beam core density in the transverse plane and decrease in density in the longitudinal plane are the expected beam cooling signals with a wedge absorber. 
ures 5.21-5.24, the upstream MC recon distribution occupies a larger volume than the upstream data distribution. This causes discrepancies in the density estimates and transmission ( $87 \%$ in data and $82 \%$ in $\mathrm{MC}$ recon). In addition, the $\mathrm{MC}$ recon emittance is farther from the equilibrium, which leads to a larger increase in density. In the longitudinal plane the kernel density estimator estimates the density in the one-dimensional energy phase-space (MICE in its final configuration did not have an RF cavity; for this reason, the longitudinal motion in this thesis is described using the energy coordinate); the constant density contour reduces to a constant density value, and the volume is the energy that corresponds to that density. As a crosscheck, the longitudinal density versus the average beam radius is compared with the energy distributions shown in Figures 5.20a and 5.20b. The distribution plots (in agreement with the longitudinal density versus beam radius plots) display heating in the longitudinal plane (broadening of the energy distribution from upstream to downstream of the wedge absorber). This, combined with the cooling effect in the transverse plane is a demonstration of the reverse emittance exchange effect: $19 \%$ transverse core density increase and $29 \%$ longitudinal core density reduction in data (29\% transverse core density increase and $38 \%$ longitudinal core density reduction in $\mathrm{MC}$ recon).

\subsection{Summary}

MICE simulated and experimental data for two absorber configurations (flat and wedge-shaped absorbers) were studied. KDE was used to estimate the phasespace distributions at the upstream and downstream tracker reference planes. The phase-space density information was used to compute the volume of a given constant density contour in 4D phase space using the Monte Carlo volume calculation technique. The contour density values (a total of 1000 contours) were then plotted against the fourth root of the contour volumes (average beam radii) to analyze the 
beam behavior in the transverse and longitudinal planes for the $\mathrm{LiH}$ and the wedge absorber datasets. An increase in the transverse contour density at low beam radii (beam core) was observed as a signal of transverse beam cooling. A reduction of longitudinal core density was observed as a signal of longitudinal heating. In addition, the wedge-shaped absorber was observed to introduce dispersion into the beam and demonstrate reverse emittance exchange: transverse beam cooling and longitudinal heating due to the beam acquiring dispersion downstream of the absorber. 


\section{CHAPTER 6 CONCLUSIONS AND SUMMARY}

The kernel density estimation (KDE) technique estimated the density without an assumption about the functional form of the data; each data point got to "speak for itself" when the distribution of the underlying dataset was being estimated. The KDE technique was applied to the experimental data collected with different absorber configurations in the Muon Ionization Cooling Experiment experiment (MICE). The single-particle measurement approach in MICE enabled each muon to contribute to the beam cooling signal; this combined with the power of the KDE technique led to a detailed understanding of the MICE cooling signal, including the migration patterns of each muon as the muon beam traversed a $\mathrm{LiH}$ or a wedge absorber.

In addition to MICE experimental data, the KDE technique was applied to various simulated beam distributions. The performance of the KDE technique was first studied in a simulated quadrupole focusing channel, where (per Liouville's theorem) in the absence of material, the estimated density was conserved. It was then applied to a simulated beam distribution traversing a matched solenoidal channel using a so-called MICE "baseline lattice"; all the magnets were operating at nominal currents, and a flat $\mathrm{LiH}$ absorber was placed in the channel. No muon transmission loss was present in this particular cooling channel, and using the KDE technique, transverse beam cooling was demonstrated in the form of an increase in the beam core phase-space density and reduction in the beam core phase-space volume. Then, the KDE technique was used to estimate the density of a muon beam traversing a simulated lattice where the magnet coil currents deviated from those of the "baseline lattice": the matching coils downstream of the flat LiH absorber were turned off, causing the beam distribution to become mismatched at the exit of the absorber and 
the muon transmission loss to drop below $90 \%$. The same types of studies were done for a simulated cooling channel containing a polyethylene wedge-shaped absorber. A wedge absorber was capable of exchanging the transverse and longitudinal planes. A simulated muon beam distribution traversing the wedge absorber was studied, and both direct and reverse emittance exchange were observed in simulation.

In Chapter 5, the kernel density estimator was applied to MICE experimental data for two absorber configurations: flat and wedge-shaped absorbers. The phasespace distributions at the upstream and downstream tracker reference planes were estimated. In addition, the volume inside a given constant density contour in 4D phase space was computed using the Monte Carlo volume calculation approach. The computed phase-space volume information was then used in calculating the average beam radii of each phase-space contour. The contour density values were then plotted against the average beam radii to analyze the beam behavior in the transverse and longitudinal planes for the flat $\mathrm{LiH}$ and the polyethylene wedge absorbers. In the case of the flat $\mathrm{LiH}$ absorber, transverse beam cooling was observed resulting in a $12 \%$ increase in the transverse density at low beam radii (beam core). A reduction of longitudinal core density of $14 \%$ was also observed indicating longitudinal heating. In the case of the wedge-shaped absorber, the beam was observed to acquire positionenergy correlation as a sign of reverse emittance exchange: the transverse core density increased by $19 \%$ and the longitudinal core density reduced (heating) by $29 \%$.

Transverse muon ionization cooling as demonstrated using the KDE technique is essential to a future neutrino factory. In addition to cooling in the transverse direction, muon collider needs longitudinal cooling; this leads to a muon collider scheme which consists of a number of flat and wedge-shaped absorbers present in the cooling channel. One of the main challenges of such muon collider scheme, however, is that the muon bunches become shorter than necessary for the required high-luminosity. 
The solution to this is the reverse emittance exchange which has been demonstrated in this thesis via the novel application of the kernel density estimation technique to the MICE data. 


\section{BIBLIOGRAPHY}

[1] Choubey, S. et al., "International Design Study for the Neutrino Factory, Interim Design Report." Nova Science Publishers, Inc., 2011, inspirehep.net/record/ 900279.

[2] Ankenbrandt, C. M. et al., "Status of Muon Collider Research and Development and Future Plans," Physical Review Accelerator and Beams, vol. 2, 1999, pp. 081001, doi:10.1103/PhysRevSTAB .2.081001.

[3] Brown, L. M., "The Idea of the Neutrino," Physics Today, vol. 31, 1978, pp. 23-28, doi:10.1063/1.2995181.

[4] Giunti, C., Kim, C., W., Fundamentals of Neutrino Physics and Astrophysics, Oxford University Press, 2007.

[5] Cowan, C. L., Reines, F., Harrison, F. B., Kruse, H. W., McGuire, A. D., "Detection of the free neutrino: A Confirmation," Science, vol. 124, 1956, pp. 103-104, doi:10.1126/science.124.3212.103.

[6] "Celebrating the Neutrino," Los Alamos Science, vol. 25, 1997, pp. 1-12.

[7] Danby, G., Gaillard, J. M., Goulianos, K., Lederman, L. M., Mistry, N., Schwartz, M, Steinberger, J., "Observation of high-energy neutrino reactions and the existence of two kinds of neutrinos," Physical Review Letters, vol. 9, 1962, pp. 36-44.

[8] Kodama, K. et al., "Observation of tau neutrino interactions," Physical Review Letters, vol. B504, 2001, pp. 218-224, doi:10.1016/S0370-2693(01)00307-0.

[9] Beringer. J. et al., (Particle Data Group), "Review of Particle Physics," Physical Review D, vol. 86, 2012, pp. 010001, doi:10.1103/PhysRevD.86.010001.

[10] Adrian-Martinez, S. et al., "Letter of intent for KM3NeT 2.0," Journal of Physics, vol. G43, 2016, pp. 084001, doi:10.1088/0954-3899/43/8/084001.

[11] Davis, R., "Solar neutrinos. II: Experimental," Physical Review Letters, vol. 12, 1964, pp. 303-305, doi:0.1103/PhysRevLett.12.303.

[12] Hirata, K. S. et al., "Observation of B-8 Solar Neutrinos in the KamiokandeII Detector," Physical Review Letters, vol. 63, 1989, pp. 16, doi:10.1103/ PhysRevLett.63.16.

[13] Anselmann, P. et al., "Solar neutrinos observed by GALLEX at Gran Sasso," Physical Review Letters, vol. B285, 1992, pp. 376-389, doi:10.1016/ 0370-2693(92) 91521-A.

[14] Abazov, A. I. et al., "Search for neutrinos from the Sun using the reaction ${ }^{71} \mathrm{Ga}\left(\nu_{e}, e^{-}\right)^{71} \mathrm{Ge}, "$ Physical Review Letters, vol. 67, 1991, pp. 3332-3335, doi:10.1103/PhysRevLett.67.3332.

[15] Ahmad, Q. R. et al., "Measurement of the Rate of $\nu_{e}+d \rightarrow p+p+e^{-}$Interactions Produced by ${ }^{8} B$ Solar Neutrinos at the Sudbury Neutrino Observatory" Physical Review Letters, vol. 87, 2001, pp. 071301, doi:10.1103/PhysRevLett. 87.071301. 
[16] Fukuda, Y. et al., "Measurement of the Solar Neutrino Energy Spectrum Using Neutrino-Electron Scattering," Physical Review Letters, vol. 82, 1999, pp. 24302434, doi:10.1103/PhysRevLett.82.2430.

[17] Fogli, G. L., Lisi, E., Marrone, A., Scioscia, G., "Super-Kamiokande atmospheric neutrino data, zenith distributions, and three-flavor oscillations," Physical Review Letters, vol. 59, 1998, pp. 033001, doi:10.1103/PhysRevD.59.033001.

[18] Boscolo, M., Huber, P., Long, K., "Muons cooled for action," CERN Courier, IOP Publishing, vol. 58, 2018, pp. 19-23, https://cerncourier.com/ muons-cooled-for-action/.

[19] Ding, X., Berg, J. S., Cline, D., Kirk, H. G., "Optimization of a mercury jet target for a neutrino factory or a muon collider," Physical Review Special Topic Accelerators and Beams, vol. 14, 2011, pp. 111002, doi:10.1103/PhysRevSTAB. 14.111002.

[20] Gregoire, G. et al., "Proposal to the Rutherford Appleton Laboratory: an international muon ionization cooling experiment (MICE)," MICE Collaboration Meeting CM4, 2003, MICE-Note-21. http://mice.iit.edu/mnp/MICE0021. pdf

[21] Snopok, P., Johnstone, C., "Recent Progress in Muon Collider Storage Ring Lattice Design," 3rd International Conference in Physics and Control, 2007.

[22] Gomez-Cadenas, J. J., "The physics case of the Neutrino Factory," Journal of Physics: Conference Series, vol. 136, 2008, pp. 022023, doi:10.1088/ $1742-6596 / 136 / 2 / 022023$.

[23] de Gouvea, A. et al., "Working Group Report: Neutrinos," Proceedings, 2013 Community Summer Study on the Future of U.S. Particle Physics: Snowmass on the Mississippi (CSS2013), 2013, FERMILAB-CONF-13-479-E, arXiv:1310.4340.

[24] Delahaye, J. P. et al., "A Staged Muon Accelerator Facility for Neutrino and Collider Physics," Fermilab Report, FERMILAB-CONF-14-211-APC-E-T, 2015, arXiv:1502.01647.

[25] Zimmermann, F. "Future Colliders for Particle Physics - "Big and Small"," Proceedings of the Third European Advanced Accelerator Concepts Workshop, EAAC2017, 2018, arxiv:1801.03170v1.

[26] Lee, S. Y., Accelerator Physics, 3rd ed., World Scientific Publishing, 2012.

[27] Kaplan, D. M., MICE and MAP Collaborations "Muon Colliders and Neutrino Factories," Proceedings of 3rd International Conference on New Frontiers in Physics, ICNFP, vol. 95, 2015, pp. 03019, doi :10.1051/epjconf/20159503019.

[28] Neuffer, D., "Principles and applications of muon cooling," Particle Accelerator, vol. 14, 1983, pp. 75-90, FERMILAB-FN-0378.

[29] Stratakis, D., Palmer, R., "Rectilinear six-dimensional ionization cooling channel for a muon collider: A theoretical and numerical study," Physical Review Special Topics Accelerators and Beams, vol. 18, 2015, pp. 031003. 
[30] Boscolo, M. et al., "Low emittance muon accelerator studies with production from positrons on target," Physical Review Accelerators and Beams, vol. 21, 2018, pp. 061005, doi:10.1103/PhysRevAccelBeams.21.061005.

[31] Mikhailichenko, A. A., Zolotorev, M. S., "Optical Stochastic Cooling," SLAC publication, SLAC-PUB-6272, 1993.

[32] Palmer R. et al., "A Complete Scheme of Ionization Cooling for a Muon Collider," Conference Proceedings, vol. C070625, 2007, pp. 3193, doi:10.1109/PAC. $2007.4440712,10.2172 / 921988$.

[33] Alsharoa, M. M. et al., "Recent progress in neutrino factory and muon collider research within the Muon collaboration," Physical Reviews Special Topics Accelerators and Beams, vol. 6, 2003, pp. 081001.

[34] Courant, E., D., Snyder, H., S., "Theory of the Alternating-Gradient Synchrotron," Annals of Physics, vol. 281, 2000, pp. 360-408, doi:10.1006aphy.2000.6012.

[35] Chao, A. W., and Mess, C. H., Tigman, M., Zimmermann, F., eds. Handbook of Accelerator Physics and Engineering, World Scientific, 2nd ed., 2013.

[36] The $\mu^{+} \mu^{-}$Collider Collaboration, " $\mu^{+} \mu^{-}$Collider - A Feasibility Study," Snowmass Workshop Proceedings, Fermilab-Conference-96-092, 1996.

[37] http://public.muonsinc.com/

[38] Booth, C. N., Hodgson, P. et al., "The design, construction and performance of the MICE target," Journal of Instrumentation, vol. 8, 2013, pp. 03006, doi: $10.1088 / 1748-0221 / 8 / 03 /$ P03006.

[39] Bonesini, M. et al., "The MICE Muon Beam on ISIS and the beamline instrumentation of the Muon Ionization Cooling Experiment," Journal of Instrumentation, vol. 7, 2012, pp. P05009, doi:10.1088/1748-0221/7/05/P05009.

[40] Soler, P. et al., "Pion contamination in the MICE muon beam," Journal of Instrumentation, vol. 11, 2016, pp. P03001, doi:10.1088/1748-0221/11/03/ P03001.

[41] Drielsma, F. et al., "Electron-Muon Ranger: performance in the MICE Muon Beam," Journal of Instrumentation, vol. 10, 2015, pp. P12012, doi:10.1088/ 1748-0221/10/12/P12012.

[42] Ellis, M. et. al., "The design, construction and performance of the MICE scintillating fibre trackers," Nuclear Instrumentation Methods, vol. A659, 2011, pp. 136-153, doi:10.1016/j.nima.2011.04.041.

[43] Tunnell, C. D., Rogers, C. T., "MAUS: MICE Analysis User Software," Proceedings of 2nd International Particle Accelerator Conference, vol. C110904, 2011, pp. 850-852, report number:IPAC-2011-MOPZ013.

[44] Hunt, C. J., "High precision track reconstruction and first emittance measurements in the MICE step IV cooling channel," Imperial College London, 2017. https://spiral.imperial.ac.uk/handle/10044/1/44974.

[45] Silverman, B. W., Density Estimation for Statistics and Data Analysis, 1st ed., Monographs on Statistics and Applied Probability, Chapman and Hall, 1986. 
[46] Hansen, B. E., Lecture Notes on Nonparametrics, University of Wisconsin, 2009. https://www.ssc.wisc.edu/ bhansen/718/NonParametrics1.pdf

[47] Scott, D. W., Multivariate Density Estimation: Theory, Practice, and Visualization, 2nd ed., John Wiley and Sons Inc, 2015.

[48] Mohayai, T. A. et al., "Simulated Measurements of Cooling in Muon Ionization Cooling Experiment," Proceedings of 7th International Particle Accelerator Conference, 2016, pp. TUPMY011, doi:10.18429/JACoW-IPAC2016-TUPMY011.

[49] T. Roberts, "G4beamline User's Guide", Muons Inc (2013).

http://www . muonsinc. com

[50] Rogers, C., T., "xboa:: A multiparticle tracking postprocessor library for accelerator physicsists," SourceForge. N.p., 18 Sept. 2010. Web. 21 June 2018.

https://code. launchpad.net/ chris-rogers/xboa/sourceforge

[51] Stewart, J., "Calculus," 5th ed., pp. 1119, Thomson Brooks/Cole, 2003.

[52] Mohayai, T. A. et al., "Novel Application of Density Estimation Techniques in Muon Ionization Cooling Experiment," Proceedings of the APS Division of Particles and Fields Meeting, APSDPF2017, 2017, arxiv:1710.04780.

[53] Mahalanobis, P. C., "On the generalised distance in statistics", Proceedings of the National Institute of Sciences of India, vol. 2,1936, pp. 49-55.

[54] Mohayai, T. A. et al., "First Demonstration of Ionization Cooling in MICE," Proceedings of the International Particle Accelerator Conference, IPAC2018, 2018, arxiv:1806.01807.

[55] Brun, R., and Rademakers, F., "ROOT: An Object Oriented Data Analysis Framework," Proceedings AIHENP'96 Workshop, Nuclear Instrumentation Methods, vol. A389, 1996, pp. 81-86. doi:10.1016/S0168-9002(97)00048-X.

[56] Dobbs, A., Long, K. et al., "The Reconstruction Software for the Muon Ionization Cooling Experiment Trackers," Journal of Physics, Conference Series, vol. 513, 2014, pp. 022008. 